\title{
LLUVIA, ESCURRIMIENTO Y PRODUCCIÓN DE SEDIMENTOS EN UNA MICROCUENCA AGRÍCOLA DEL SISTEMA DE TANDILIA
}

\author{
TESIS DOCTORAL
}

María Guadalupe Ares

Tesista

Dr. Marcelo Varni

Director

\section{Dr. Celio Chagas}

Co Director

La Plata, 27 de marzo de 2014 


\section{AGRADECIMIENTOS}

A mis directores de tesis: Dr Marcelo Varni y Dr Celio Chagas, por todo el tiempo que han dedicado durante todos estos años a mi formación, por compartir conmigo sus conocimientos y su experiencia profesional, y por su confianza.

Al Dr Luis Vives, por su confianza en todos estos años.

A Ing (MsSc) Georgina Cazenave y a la Dra llda Entraigas, por su apoyo permanente e incondicional en este camino de la investigación, por su colaboración, por sus conocimientos compartidos, por sus consejos y por alentarme a continuar.

A la empresa Guivor S.A., por permitir la instalación del instrumental de medición en el establecimiento "Santa María", para la realización de este trabajo de Tesis. Al personal de esta empresa, por su colaboración y su excelente predisposición para atender mis consultas y solicitudes: Ing. Andrés Alonso, Ing. Valeria Mesa, Ing. Eduardo Bordino, al Sr Federico Melendi y a la Sra María José Bazzano.

A la empresa Cacique Negro S.A.A.G por permitir el ingreso al establecimiento "Cerro del Águila" para la realización de diferentes muestreos. Agradezco a su administrador, $\mathrm{Sr}$ Carlos Rodríguez, por la excelente predisposición para colaborar y para responder a mis consultas y solicitudes.

A los propietarios del establecimiento "Los Manantiales" y a su administrador, Sr José Imas, por permitir la realización de muestreos, y por su colaboración y su atención ante todas las consultas y solicitudes durante estos años de trabajo.

A los profesionales del Laboratorio de Análisis Químicos de Aguas del Instituto de Hidrología de Llanuras (IHLLA): Ing. Daniel Arias, Lic. Fátima Altolaguirre y Téc. Natalia de Líbano, por su colaboración en el procesamiento y análisis de las muestras de agua.

A los técnicos del IHLLA: Enrique Queupán, Joaquín Rodríguez y Matías Silicani, por su colaboración en las tareas de campo, recolección de muestras y datos, y mantenimiento de los equipos de monitoreo.

A los Dres Mauro Holzman y Raúl Rivas, por su colaboración en el procesamiento de la imagen satelital Spot-5.

A la Dra Fernanda Gaspari, por atender a mis consultas.

A todos los Investigadores y Becarios del IHLLA, por estar dispuestos a ayudarme en todo momento y en lo que fuera necesario, por interesarse en mi trabajo, en mi avance y por compartir la alegría de los logros alcanzados.

Y a mi familia y amigas, por estar a mi lado y por el tiempo que les quité...

¡Gracias a todos!

Este trabajo fue financiado parcialmente por los proyectos UBACyT 709 y 937 (periodo 2011-2014) y por subsidios de la Secretaría de Ciencia, Arte y Tecnología de la UNCPBA otorgados al Núcleo de Actividades Científicas y Tecnológicas de Investigaciones en Hidrología de Llanuras (NACT-IHLLA). 


\section{ÍNDICE}

1. INTRODUCCIÓN.

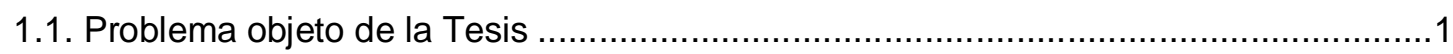

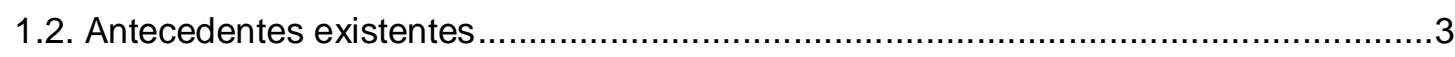

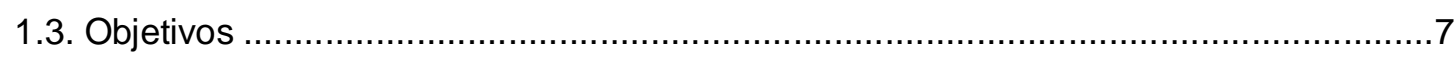

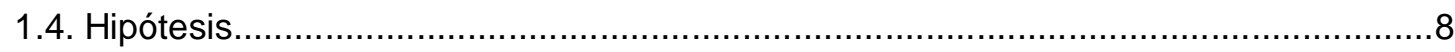

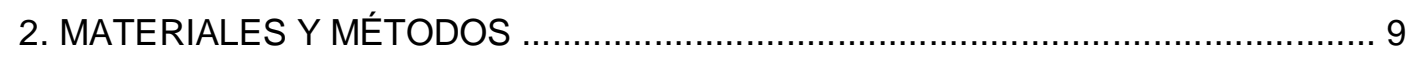

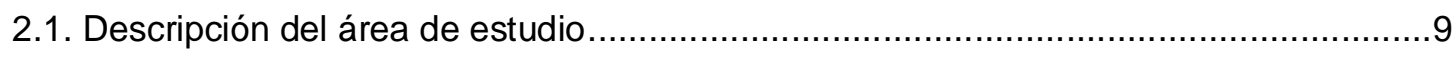

2.2. Precipitaciones y variables relacionadas: registro, cálculos y caracterización para el

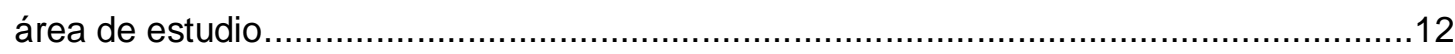

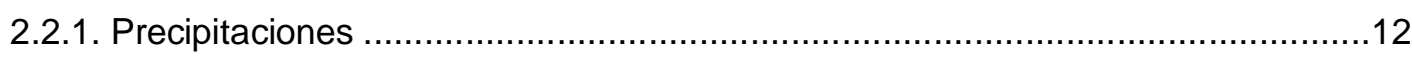

2.2.2 Energía de la precipitación, intensidades máximas en 10 y 30 minutos e índice

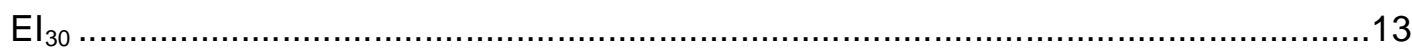

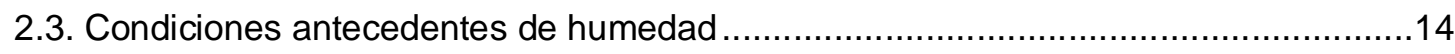

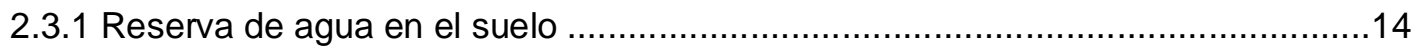

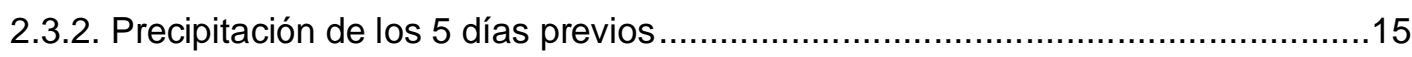

2.4. Variables hidrológicas: registro y cálculos realizados ............................................. 15

2.5. Muestreos de agua de escurrimiento durante las crecidas ....................................18

2.5.1. Producción de sedimentos estimada...............................................................19

2.6. Parámetros de la Ecuación Universal de Pérdida de Suelo Modificada .....................19

2.6.1. Factor de escurrimiento ............................................................................ 19

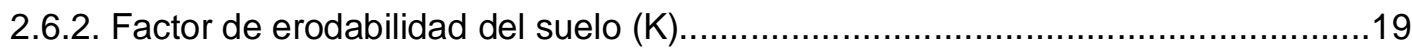

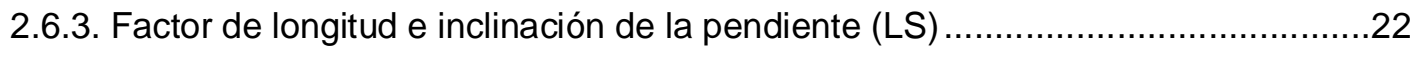

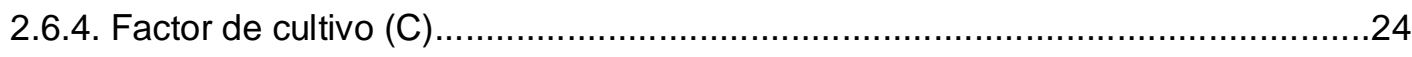

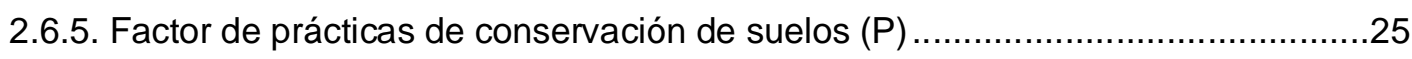

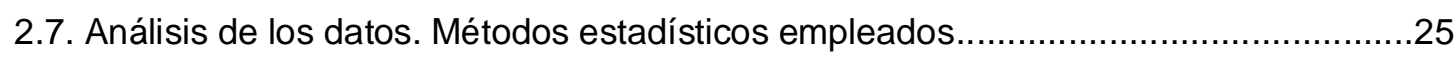

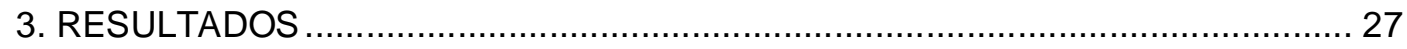

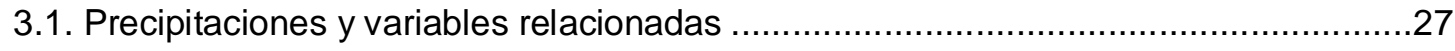

3.1.1. Caracterización de las precipitaciones en la zona.......................................27

3.1.1.a. Análisis de dobles masas...............................................................27

3.1.1.b. Distribución mensual de las precipitaciones ...........................................27

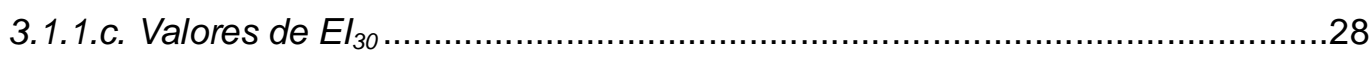

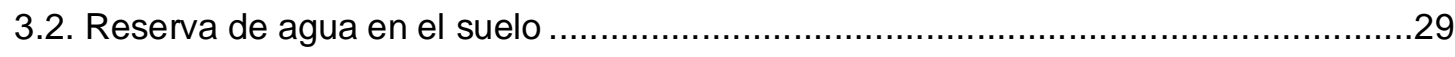

3.3. Eventos Iluvia-escurrimiento-producción de sedimentos registrados ........................30

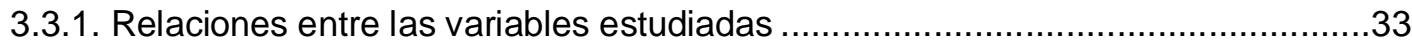


3.3.1.a. Análisis a escala de eventos individuales.

3.3.1.b. Análisis estacional: períodos de baja a media cobertura y de alta cobertura36 3.4. Agrupamiento de los eventos de acuerdo a la interacción lluvia-escorrentíaproducción de sedimentos mediante análisis de cluster .39

3.5. Producción de sedimentos estimada .42

3.5.1. Relaciones entre la producción de sedimentos estimada y las variables estudiadas .42

3.5.1.a. Análisis a escala de eventos individuales.

3.5.1.b. Análisis a escala estacional: períodos de baja a media cobertura y de alta cobertura ...44

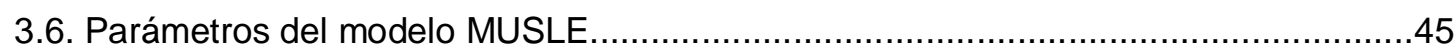

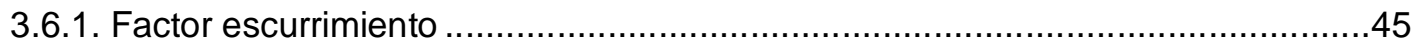

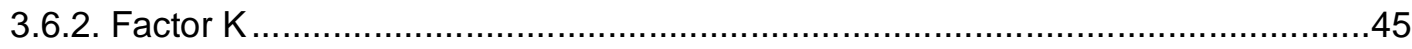

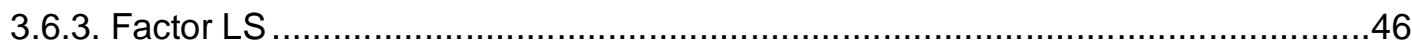

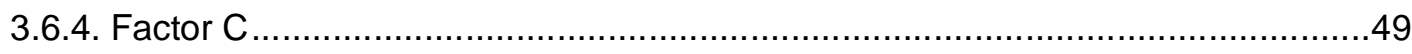

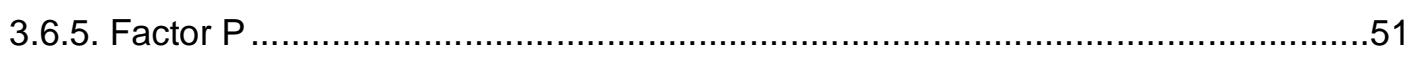

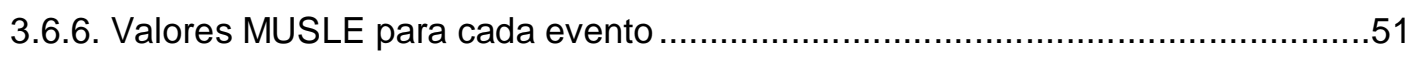

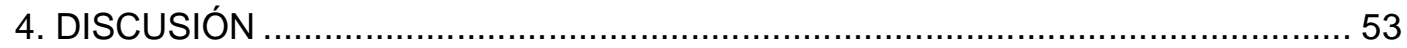

4.1. Precipitaciones, erosividad de las precipitaciones y reserva de agua en el suelo en el período estudiado .53

4.2. Variabilidad en la respuesta a las precipitaciones y a la producción de sedimentos ..54

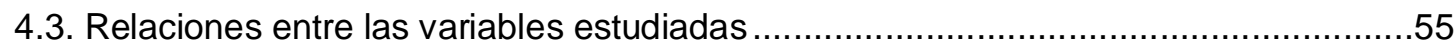

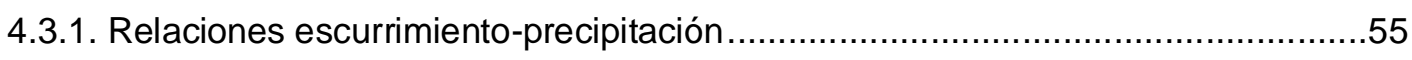

4.3.1.a. Análisis a escala de eventos individuales..................................................55

4.3.1.b. Análisis estacional: períodos de baja a media cobertura y de alta cobertura58

4.3.2. Relaciones entre CSS, variables del escurrimiento y variables de la preciptación

4.3.2.a. Caracterización de los muestreos discreto y compuesto .............................63

4.3.2.b. Análisis a escala de eventos individuales................................................66

4.3.2.c. Análisis estacional: períodos de baja a media cobertura y de alta cobertura72

4.3.3. Relación entre humedad antecedente, escurrimiento y CSS ............................73

4.4. Agrupamiento de los eventos de acuerdo a la interacción lluvia-escorrentíaproducción de sedimentos mediante análisis de cluster ...............................................75

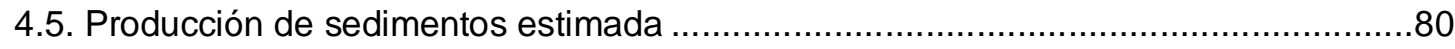

4.5.1. Relaciones entre la producción de sedimentos estimada y las variables

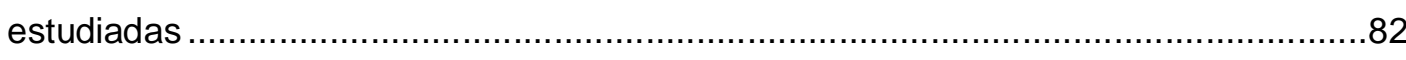

4.5.1.a. Análisis a escala de eventos individuales.............................................82 
Lluvia, escurrimiento y producción de sedimentos en una microcuenca agrícola del Sistema de Tandilia

4.5.1.b. Análisis estacional: períodos de baja a media cobertura y de alta cobertura88

4.6. Estimaciones de la producción de sedimentos a partir del modelo MUSLE ...............89

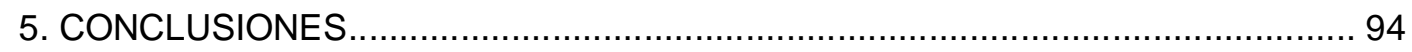

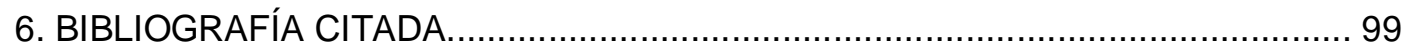

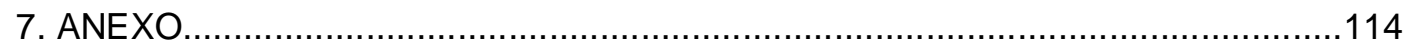




\section{ÍNDICE de TABLAS}

Tabla 3.1. Características generales de los eventos registrados en la microcuenca del arroyo Videla. 32

Tabla 3.2. Coeficientes de Correlación de Pearson entre las variables analizadas. La correlación es significativa al nivel 0,01 para los números en negrita y al nivel 0,05 para los números en negrita cursiva. .34

Tabla 3.3. Coeficientes de Correlación de Pearson entre las variables analizadas considerando los eventos del período de baja a media cobertura.

Tabla 3.4. Coeficientes de Correlación de Pearson entre las variables analizadas considerando los eventos del período de alta cobertura. 38

Tabla 3.5. Promedios y coeficientes de variación (CV) de las variables correspondientes a los casos incluidos dentro de cada grupo obtenido a través del análisis de cluster.

Tabla 3.6. Producción total de sedimentos estimada, expresada en toneladas correspondientes a los 17 eventos registrados en la microcuenca bajo estudio.

Tabla 3.7. Coeficientes de Correlación de Pearson entre las variables analizadas y la producción de sedimentos estimada.

Tabla 3.8. Coeficientes de Correlación de Pearson entre las variables analizadas y la producción de sedimentos estimada para los períodos de alta cobertura y de media a baja cobertura.

Tabla 3.9. $\mathrm{K}$ expresados en unidades métricas (Ton ha $\mathrm{h}$ (ha $\mathrm{MJ} \mathrm{mm})^{-1}$ ) obtenidos para las observaciones de suelos realizadas en las unidades ambientales delimitadas en la microcuenca. Valores del factor asignados a dichas unidades y superficie asociada. Factor $\mathrm{K}$ ponderado para la microcuenca bajo estudio.

Tabla 3.10. Pendiente promedio, área, longitud de la pendiente $(\lambda)$ y valor de LS para las áreas de pendientes menores que $9 \%$ y mayores que $9 \%$ considerando sectores inferior, medio y superior de la microcuenca bajo estudio.

Tabla 3.11. Planilla de cálculo del factor $\mathrm{C}$, con detalle de fecha de la crecida, usos del suelo, etapa en la que se encontraban los cultivos y valor de relación de pérdida de suelo asociado para cada lote correspondiente a la microcuenca. Valor de C ponderado para la crecida..

Tabla 3.12. Valores de $\mathrm{C}$ ponderados correspondientes a los 17 eventos analizados en la microcuenca bajo estudio.

Tabla 3.13. Valores de pérdida de suelo, expresados en toneladas, calculados mediante el modelo MUSLE para los 17 eventos analizados en la microcuenca bajo estudio. .52 
Tabla 4.1. Intensidad promedio de las precipitaciones en 10 y 30 minutos correspondiente a la totalidad de las tormentas registradas en el período de media a baja cobertura $(n=11)$ y en el período de alta cobertura $(n=33)$.

Tabla 4.2. Momentos de muestreo discreto y compuesto, en relación a la magnitud de la curva de escurrimiento, y eventos asociados a cada caso.

Tabla 4.3. Parámetros de las relaciones de regresión lineal simple entre la concentración del muestreo discreto y el escurrimiento, entre la concentración del muestreo discreto y el caudal base y entre la concentración del muestro discreto y el coeficiente de escurrimiento.

Tabla 4.4. Parámetros de las relaciones exponenciales entre la concentración del muestreo discreto correspondiente al período de media a baja cobertura y: la precipitación total, el índice $\mathrm{El}_{30}$, la energía de la precipitación y la intensidad máxima en 30 minutos. 72

Tabla 4.5. Parámetros de las ecuaciones de regresión lineal entre el escurrimiento y el caudal base, entre la concentración de sólidos suspendidos del muestreo discreto y el caudal base y entre la concentración de sólidos suspendidos del muestreo compuesto y el caudal base.

Tabla 4.6. Estabilidad estructural por el método de Le Bissonnais (1996) e hidrofobicidad por el método citado por Chenu et al. (2000), correspondientes a una muestra compuesta de los sitios de observaciones de suelos para el cálculo del factor $\mathrm{K}$.

Tabla 4.7. Resultados de ensayos de simulación de lluvia realizados en el área de estudio.

Tabla 4.8. Duración del muestreo compuesto en relación al tiempo total de la crecida expresada como valor porcentual, correspondiente a los 17 eventos analizados en el área de estudio.

Tabla 4.9. Parámetros de las ecuaciones de ajuste lineal por tramos entre la producción de sedimentos estimada y el caudal pico.

Tabla 7.1. Planilla de cálculo del factor C. Detalle de fecha de la crecida, usos del suelo, etapa en la que se encontraban los cultivos y valor de relación de pérdida de suelo asociado para cada lote correspondiente a la microcuenca. Valor de $C$ ponderado para la crecida..114 Tabla 7.1 (cont). Planilla de cálculo del factor C. Detalle de fecha de la crecida, usos del suelo, etapa en la que se encontraban los cultivos y valor de relación de pérdida de suelo asociado para cada lote correspondiente a la microcuenca. Valor de C ponderado para la crecida.. 115

Tabla 7.1 (cont). Planilla de cálculo del factor C. Detalle de fecha de la crecida, usos del suelo, etapa en la que se encontraban los cultivos y valor de relación de pérdida de suelo asociado para cada lote correspondiente a la microcuenca. Valor de C ponderado para la crecida...... 
Lluvia, escurrimiento y producción de sedimentos en una microcuenca agrícola del Sistema de Tandilia

Tabla 7.1 (cont). Planilla de cálculo del factor C. Detalle de fecha de la crecida, usos del suelo, etapa en la que se encontraban los cultivos y valor de relación de pérdida de suelo asociado para cada lote correspondiente a la microcuenca. Valor de C ponderado para la

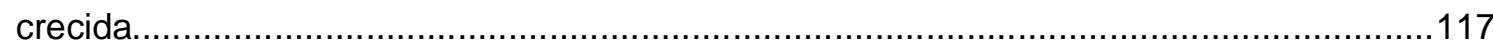




\section{ÍNDICE de FIGURAS}

Figura 2.1.a) Ubicación de la microcuenca en estudio en la cuenca superior del arroyo del Azul, estaciones de registros pluviométricos (Trapenses y Cerro del Águila) y meteorológicos (Servicio Meteorológico Nacional, SMN). b) Detalle de la microcuenca y ubicación de estación de monitoreo de caudales y muestreo de agua de crecidas.

Figura 2.2. Precipitaciones medias mensuales correspondientes al período 1901-2012, registradas en la estación Azul Aero del Servicio Meteorológico Nacional.

Figura 2.3. Erosividad mensual de las precipitaciones de la localidad de Azul, calculadas según la metodología propuesta por Wischmeier y Smith (1978) ......................................11

Figura 2.4. Estación meteorológica "Cerro del Águila" .........................................................13 Figura 2.5. a) Estación de monitoreo de alturas y de agua de escurrimiento. b) Detalle de muestreador automático de agua y sedimentos.

Figura 3.1. Diagrama del método de dobles masas: comparación de las precipitaciones mensuales acumuladas de las estaciones Cerro del Águila y Trapenses. 28 Figura 3.2. Precipitaciones mensuales de los años 2011 y 2012 y precipitaciones medias mensuales correspondientes al período 1972-2012 registradas para el área de estudio......28 Figura 3.3. Valores mensuales del índice $\mathrm{El}_{30}$ correspondientes a los años 2011 y 2012 , comparados con los valores calculados por Ares et al. (2010) para la localidad de Azul en el período 1995-2007.

Figura 3.4. Valores medios diarios mensuales de reserva de agua en el suelo correspondientes a la microcuenca bajo estudio ( $\mathrm{y}$ sus respectivos coeficientes de variación) calculados para los años 2011 y 2012.

Figura 3.5. Dendrograma del análisis de cluster sobre los datos de precipitación, escurrimiento y variables asociadas a estos y concentración de sólidos suspendidos. ........40 Figura 3.6. Modelo digital del terreno obtenido a partir de la digitalización de las curvas de nivel correspondientes a las cartas del Instituto Geográfico Militar, hojas 3760-22-1 "Base Azopardo" y 3760-22-3 "Chillar". Elevación expresada en metros sobre el nivel del mar. Áreas de subdivisión de la microcuenca de acuerdo con sus características físicas: a: sector superior; b: sector medio; c: sector inferior.

Figura 3.7. Red de drenaje de la microcuenca en sectores superior (a), medio (b) e inferior (c), a partir de la cual se calculó la longitud total de las vaguadas para obtener el factor LS

Figura 3.8. Mapa de pendientes correspondiente a la microcuenca bajo estudio. 48 
Figura 4.1. Evapotranspiración de referencia media mensual calculada para el período 20012007 correspondiente a la localidad de Azul (Tomado de Ares (2010)). .54

Figura 4.2. Relación entre el escurrimiento superficial y la precipitación total para los 17 eventos estudiados en la microcuenca del arroyo Videla.

Figura 4.3. Relación entre el escurrimiento superficial y el índice $\mathrm{El}_{30}$ para los 17 eventos estudiados en la microcuenca del arroyo Videla.

Figura 4.4. Relación entre los valores de CN calculados en función de la lámina de agua precipitada para los 17 eventos registrados en la microcuenca bajo estudio. .58

Figura 4.5. Relación entre el escurrimiento y la reserva de agua en el suelo del día previo a la precipitación para el período de media a baja cobertura $(n=5)$.

Figura 4.6. Relación entre el escurrimiento y la intensidad máxima de la precipitación en 10 minutos, considerando los casos correspondientes al período de alta cobertura.

Figura 4.7. Relación entre el escurrimiento y la intensidad máxima de la precipitación en 10 minutos, considerando los casos correspondientes al período de media a baja cobertura...62 Figura 4.8. Relación entre el escurrimiento y la precipitación considerando los casos correspondientes al período alta cobertura.

Figura 4.9. Relación entre el escurrimiento y la precipitación considerando los casos correspondientes al período de media a baja cobertura.

Figura 4.10. Alturas correspondientes al evento registrado el 05/03/2012. Momentos de recolección de muestras, en este caso, entre el pico de escurrimiento y su descenso.........65 Figura 4.11. Alturas correspondientes al evento registrado el 17/05/2012. Momentos de recolección de muestras, en este caso, en la fase de ascenso de escurrimiento.

Figura 4.12. Relación entre la concentración de sólidos suspendidos del muestreo discreto y el caudal superficial medio para los 17 eventos estudiados en la microcuenca del arroyo Videla.

Figura 4.13. Relación entre la concentración de sólidos suspendidos del muestreo discreto y la intensidad máxima de la precipitación en 30 minutos para los 17 eventos estudiados en la microcuenca del arroyo Videla.

Figura 4.14. Relación entre la concentración de sólidos suspendidos del muestreo discreto y la intensidad máxima de la precipitación en 10 minutos para los 17 eventos estudiados en la microcuenca del arroyo Videla.

Figura 4.15. Relación entre la concentración de sólidos suspendidos del muestreo discreto y el índice $\mathrm{El}_{30}$ para los 17 eventos estudiados en la microcuenca del arroyo Videla. 68

Figura 4.16. Relación entre la concentración de sólidos suspendidos del muestreo discreto y el factor de escurrimiento de MUSLE para los 17 eventos estudiados en la microcuenca del arroyo Videla. 
Figura 4.17. Relación entre la concentración de sólidos suspendidos del muestreo compuesto y el coeficiente de escurrimiento.

Figura 4.18. Relación entre la concentración de sólidos suspendidos del muestreo compuesto y el caudal pico.

Figura 4.19. Relación entre la concentración de sólidos suspendidos del muestreo compuesto y el caudal superficial medio.

Figura 4.20. Relación entre la concentración de sólidos suspendidos del muestreo compuesto y el CN.

Figura 4.21. Relación entre la concentración de sólidos suspendidos del muestreo discreto y la intensidad máxima de la precipitación en 30 minutos para el período de media a baja cobertura.

Figura 4.22. Relación entre la concentración de sólidos suspendidos del muestreo discreto y la intensidad máxima de la precipitación en 30 minutos para el período de alta cobertura. ..73 Figura 4.23. Relación entre la concentración de sólidos suspendidos y la energía de la precipitación para los eventos ubicados en los grupos $A$ y $B$ del análisis de cluster $(n=11) . .76$ Figura 4.24. Relación entre la concentración de sólidos suspendidos y el caudal superficial medio de los eventos ubicados en los grupos $C$ y $D$ del análisis de cluster $(n=6)$......

Figura 4.25. Fotografías que muestran surcos formados en el área de estudio durante la tormenta del día 17/05/2012. 79

Figura 4.26. Relación entre la producción de sedimentos estimada y la intensidad máxima de la precipitación en 30 minutos para los 17 eventos registrados en la microcuenca del arroyo Videla. .83

Figura 4.27. Relación entre la producción de sedimentos estimada y el índice $\mathrm{El}_{30}$ para los 17 eventos registrados en la microcuenca del arroyo Videla.

Figura 4.28. Relación entre la producción de sedimentos estimada y el caudal pico para los 17 eventos registrados en la microcuenca del arroyo Videla.

Figura 4.29. Relación entre la producción de sedimentos estimada y el caudal pico para los 17 eventos registrados en la microcuenca del arroyo Videla. Líneas de ajuste correspondientes a los tres tramos identificados en la relación entre las variables...............84 Figura 4.30. Relación entre la producción de sedimentos estimada y el caudal superficial medio para los 17 eventos registrados en la microcuenca del arroyo Videla.......................86 Figura 4.31. Relación entre la producción de sedimentos estimada y el coeficiente de escurrimiento para los 17 eventos registrados en la microcuenca del arroyo Videla. 87 Figura 4.32. Relación entre la producción de sedimentos estimada y el factor de escurrimiento del modelo MUSLE para los 17 eventos registrados en la microcuenca del arroyo Videla. 
Figura 4.33. Relación entre la producción de sedimentos estimada y el índice $\mathrm{El}_{30}$ para el período de alta cobertura. .88

Figura 4.34. Relación entre la producción de sedimentos estimada y el índice $\mathrm{El}_{30}$ para el período de media a baja cobertura. 89

Figura 4.35. Relación entre los valores de producción de sedimentos calculados a través del modelo MUSLE y los valores de producción de sedimentos estimados a partir de los datos de concentración observados. Línea de ajuste a modelo de regresión lineal y línea teórica que describe la relación 1:1 entre las variables.

Figura 4.36 a) y b). Imágenes correspondientes a dos sectores de la ribera del curso de agua de la microcuenca bajo estudio, mostrando la zona de vegetación densa. .93 


\section{RESUMEN}

La erosión hídrica es uno de los principales procesos de degradación que afectan a las tierras agrícolas a nivel mundial. Sus efectos tienen consecuencias en el sitio donde ocurre, al disminuir la capacidad productiva de las tierras. Al mismo tiempo ocasionan externalidades, debido a la sedimentación en canales y reservorios y a la contaminación que puede provocar. En Argentina, estadísticas del año 1990 indican que la erosión afecta 30 millones de hectáreas, y recientemente se ha establecido que 650.000 hectáreas por año se incorporan al área afectada por erosión hídrica y eólica, debido a manejos inadecuados tales como deforestación, sobrepastoreo, cultivo de tierras marginales, entre otros.

La siembra directa es una práctica de manejo que constituye una alternativa para reducir el escurrimiento y la erosión en las tierras cultivables. Su introducción en nuestro país modificó la agricultura. Su adopción se produjo rápidamente junto con la introducción de cultivos transgénicos y el aumento en el uso de agroquímicos en un proceso de incremento de la superficie destinada a la agricultura (agriculturización) que se vio favorecido por la mejora en los precios de los commodities y el incremento de las precipitaciones desde la mitad de la década de los años 70. En particular, en las tierras fértiles de las sierras de Tandilia, este sistema se introdujo de manera poco sustentable. Esto incluye el sobrepastoreo de rastrojos asociado a la reducción del área destinada a la ganadería, y la escasa planificación de las rotaciones, en las cuales el cultivo de soja tiene alta participación. Además, la sembradora de siembra directa es utilizada en combinación con operaciones previas de labranza. De este modo, los cambios ocurridos han provocado la reducción en la cobertura vegetal y en el efecto protector que ejerce. Al mismo tiempo, el aumento de las precipitaciones registrado en las últimas décadas, en el contexto antes mencionado, han contribuido a incrementar el escurrimiento, y a favorecer las condiciones para la pérdida de suelo. Esta tendencia se evidencia por los desbordes recurrentes de los arroyos de la región (durante los años 1980, 1985, 1992, 2001, 2002 y 2012) y por los síntomas de erosión que pueden ser observados con frecuencia por los productores, como surcos y cárcavas, lo que determina que este proceso de degradación constituya un problema prioritario en la región de las sierras de Tandilia.

El objetivo de esta tesis fue estudiar el proceso erosión hídrica en una microcuenca ubicada en la subcuenca superior del arroyo del Azul, que se encuentra en el Sistema de Tandilia, por medio de la evaluación de la precipitación, el escurrimiento y el transporte de sedimentos en un curso de agua, como también a través de la aplicación de un modelo empírico que describa dicho proceso. 
El estudio se basó en las siguientes hipótesis:

- En el área bajo estudio existe relación entre las características de las tormentas, generación de escurrimiento superficial y producción de sedimentos

- El escurrimiento y la producción de sedimentos sufren modificaciones a lo largo del año en función de la estacionalidad de las tormentas y de la variación en el uso del suelo

- El proceso de erosión laminar resulta predominante frente al de erosión en surcos

El análisis se realizó en una microcuenca agrícola de 566 hectáreas durante 2011 y 2012 , donde se realizaron mediciones de precipitación, escurrimiento y concentración de sedimentos. Para cada evento se consideraron las variables asociadas a la precipitación: precipitación total $(P)$, duración de la precipitación (Dur), su energía cinética total $(E)$, la intensidad máxima en 10 y en 30 minutos $\left(\mathrm{I}_{10}\right.$ e $\mathrm{I}_{30}$, respectivamente) y el producto $\mathrm{El}_{30}$. $\mathrm{El}$ escurrimiento se estudió a través de la lámina de escurrimiento total (Esc), el caudal pico (Qp), el caudal superficial medio (Qsm) y el coeficiente de escurrimiento (CE). Se obtuvieron muestras de agua de escurrimiento y se calculó la concentración de sedimentos suspendidos. Asimismo, se estimaron valores de producción total de sedimentos. La humedad antecedente se analizó a través de la reserva de agua en el suelo del día previo a la precipitación y además mediante la lluvia acumulada en los 5 días previos. Se evaluaron los parámetros de la ecuación universal de pérdida de suelo modificada (MUSLE) a partir de valores de escurrimiento, características del suelo, información topográfica y datos de cultivos, y se obtuvieron valores calculados de la producción total de sedimentos.

Considerando el conjunto de los 17 eventos registrados, se encontraron correlaciones entre las variables asociadas al escurrimiento y a la precipitación, y la concentración de sedimentos suspendidos se vinculó estadísticamente con la precipitación y el escurrimiento. La dispersión registrada en la relación entre la concentración de sedimentos y las variables del escurrimiento asociadas indicaría que el proceso de pérdida de suelo tendría un comportamiento no lineal de alta variabilidad, en relación con las características de las lluvias en cuanto a su estacionalidad, que condiciona su intensidad y su erosividad.

La producción total de sedimentos estimada mostró las mejores correlaciones con Qp, Qsm, CE o el factor de escurrimiento del modelo MUSLE, y tales relaciones se ajustaron a funciones exponenciales. El análisis detallado de la relación entre la producción de sedimentos estimada y el Qp permitió identificar cambios en la respuesta de este sistema, en vinculación con los mecanismos de erosión predominantes durante el período estudiado. Estos cambios se asociarían a modificaciones en las condiciones de equilibrio de la cuenca, que ocurrirían al ser superado un valor umbral. Así, tal situación, estaría dada por una 
pérdida en la resiliencia del sistema, es decir que no se expresaría su capacidad de regulación ante las lluvias, la que estaría dada por las propiedades de los suelos como su alto contenido de materia orgánica, su alta estabilidad estructural que favorecerían las condiciones para la infiltración del agua pluvial.

Al considerar la estacionalidad en las precipitaciones y en la cobertura vegetal, fue posible identificar diferencias en los factores involucrados en la respuesta lluvia-escurrimientoconcentración y producción de sedimentos. Durante el período de alta cobertura, la $I_{30}$ sería el principal factor que condicionaría el escurrimiento, y el flujo superficial hortoniano tendría lugar en ese caso. Al mismo tiempo, $\mathrm{I}_{30} \mathrm{y}$ el escurrimiento serían los factores que actuarían en el desprendimiento y en el movimiento de los sedimentos en las pendientes.

En el período de media a baja cobertura, el flujo superficial no solamente se generaría por el mecanismo de Horton, sino que además tendrían relevancia el los mecanismos de flujo subsuperficial e infiltración desde superficie. Esto estaría asociado a las relaciones registradas entre el escurrimiento y la precipitación, su duración, energía como asimismo a aquella registrada con la reserva de agua en el suelo. La concentración de sólidos suspendidos estaría directamente asociada a la precipitación, dado que la lluvia, I30 y EI30 fueron las únicas variables que mostraron vinculaciones con la concentración de sólidos suspendidos.

Además, este análisis permitió diferenciar la variabilidad estacional en las respuestas al escurrimiento y a la producción de sedimentos. Diferencias estacionales en la cobertura, evaporación, precipitación y condiciones de humedad antecedentes controlarían la homogeneidad en dicha respuesta durante el período de media a baja cobertura, y la heterogeneidad en tal sentido en el período de alta cobertura.

Con respecto a los tipos de erosión, la erosión laminar o entre surcos sería el proceso más frecuente en la microcuenca, con menor producción de sedimentos. En tanto, la erosión en surcos sería el proceso menos frecuente, pero el responsable de las más altas pérdidas de sedimentos. Así, podría establecerse que las pérdidas más relevantes ocurrirían en una menor proporción de eventos menos frecuentes.

Se caracterizaron los cambios en los procesos dominantes a través de la relación entre la producción de sedimentos estimada y el caudal pico. Distintos valores umbral de caudal pico indicarían cambios de erosión laminar a erosión en surcos, y en modificaciones en la intensidad del proceso en surcos. Este último punto podría relacionarse con la pérdida de la capacidad de regulación antes mencionada. En esos casos, habría otros factores con mayor 
preponderancia en el escurrimiento y la producción de sedimentos, como la masa y la energía del flujo, la formación de caminos preferenciales para el escurrimiento y, vinculado a esto, la conectividad en el paisaje. Así, estos factores contribuirían al cambio en el equilibrio, y además, a la alta pérdida de suelo.

Las diferencias más altas entre la producción de sedimentos estimada mediante datos observados y los valores predichos con la ecuación universal de pérdida de suelo modificada, correspondió a los eventos con mayores pérdidas de suelo. Estas diferencias indicarían la complejidad en la respuesta a la producción de sedimentos en esos eventos, y asimismo, las mayores limitaciones de este modelo para predecir la pérdida de suelo en tales situaciones. 


\section{ABSTRACT}

Water erosion is one of the major degradation processes affecting agricultural land worldwide. It has consequences on the site where it occurs, by reducing the productive capacity of the land, and outside this area, due to sedimentation and pollution that the erosion can cause. In Argentina, statistics of 1990 show that water erosion affects 30 million hectares, and recently it has been established that 650000 hectares per year are incorporated to the area affected by water and wind erosion, because of mismanagements such as deforestation, overgrazing and cultivation of marginal and environmentally sensitive areas.

No tillage is a management practice that represents an alternative for reducing runoff and erosion in cultivated lands. In our country no tillage produced a technological change that has modified farming. Its adoption occurred rapidly with the introduction of genetically modified crops and the increased use of agrochemicals in the process of increasing the area dedicated to agriculture (agriculturization), which was aided by improved commodities prices and increased rainfall since mid-70s. Particularly, in the highly fertile lands of the Tandilia hills, no tillage system was introduced unsustainably. This includes overgrazing of stubble, associated with the decrease in the area dedicated to cattle raising, and poor planning of rotations, in which soybean cultivation has high participation. In addition, no tillage seeder is used in combination with previous tillage operations. Thus, the changes have resulted in the reduction in vegetation cover in this area and, consequently, a reduction in its protective effect. At the same time, the rainfall increase recorded in recent decades, related to the above conditions, has contributed to increasing runoff and soil susceptibility to water erosion in the area. This trend is evidenced by recurring flood events in the region (in the years 1980 , $1985,1992,2001,2002$, and 2012) and by the erosion symptoms that can often be observed in the fields by farmers, such as rills and gullies, which determine that this degradation process is a priority issue in the Tandilia area.

The aim of this thesis was to study water erosion process in a small watershed located in the upper basin of the del Azul stream, representative of the hilly region of Tandilia, by the evaluation of rainfall, runoff and sediment transport in a water course, as well as by the application of an empirical model which describes that process.

The study was based on the following hypotheses: 
-In the study area there is a relationship between storm chacateristics, surface runoff and sediment production

-Runoff and sediment production are modified along the year, in relation to storm seasonality and land use change

-Interrill erosion process prevails over rill erosion process

The study was conducted in a small agricultural watershed of 566 hectares during 2011 and 2012, where precipitation, runoff and sediment concentration were monitored. Each event was characterized with the variables associated with precipitation: its total value $(P)$, its duration (Dur), its total kinetic energy (E), its maximum intensity in 10 and in 30 minutes $\left(I_{10}\right.$ and $\mathrm{I}_{30}$ respectively), and the product $\mathrm{El}_{30}$. Runoff was analyzed through the surface runoff sheet (Esc), peak discharge (Qp), mean surface discharge (Qsm) and runoff coefficient (CE). Runoff samples were obtained and suspended sediment concentrarion was calculated. Values of total suspended sediment yield were estimated as well Antecedent moisture was studied through soil water reserve at the rainfall previous day (ResA) and through the precipitation accumulated during the previous five days ( $\mathrm{Pa} 5 \mathrm{~d})$. Parameters of the modified universal soil loss equation (MUSLE) were evaluated considering runoff values, soil characteristics, topographical information and crops data, and calculated values of total sediment production were obtained.

Considering the 17 registered events, runoff and rainfall variables were correlated, and suspended sediment concentration was statistically related to precipitation and runoff. Dispersion was registered in the relationship between sediment concentration and runoff related variables. This showed that soil loss process would have a nonlinear and highly variable behavior, related to seasonality in rainfall characteristics which conditions their intensity and erosivity.

Total sediment production estimation was better correlated with Qp, Qsm, CE or MUSLE's runoff factor, and these relationships were adjusted to exponential functions. Detailed analysis of the sediment production vs Qp relation let identify changes in the response of this system in association with prevailing erosion mechanisms during the study period. These changes could be related to modifications of the equilibrium conditions of the watershed that would occur when a threshold value is exceeded, that is, when the resilience of the system related to soil properties such as high organic matter contents and structural stability that would favor high infiltration of soils, is surpassed. 
It was possible to recognize differences about factors involved in rainfall-runoffconcentration and sediment production response, when seasonality in rainfall and vegetal cover was considered. During high cover period, the maximum intensity in 30 minutes would be the prevailing factor conditioning runoff, and the Horton overland flow type would occur. At the same time, $I_{30}$ and runoff would detach and make sediment move on hillslopes in these cases.

For the period of medium to low vegetal cover, not only Horton overland flow type would occur, but subsurface stormflow and water-table rise could generate storm runoff as well. This would be associated to the observed relationships between runoff and precipitation, its duration, its energy, and soil water reserve. Concentration of suspended solids would be directly related to precipitation, as rainfall, $\mathrm{I}_{30}$ and $\mathrm{El}_{30}$ were the only variables that showed relationships with suspended solids concentration during this period.

Furthermore, this analysis showed dissimilarities in seasonal dispersion in runoff and sediment production responses. Seasonal differences in soil cover, evaporation, precipitation and antecedent moisture conditions would control the homogeneity in that response during the period of medium to low vegetal cover, and the heterogeneity in the high cover period.

Regarding types of water erosion, sheet or interrill erosion would be the most frequent process in this small watershed, with less sediment production. Whereas, rill erosion would be the less frequent process, but responsible of the highest sediment losses. So, it could be said that the most relevant sediment losses would occur in a small proportion of less frequent events.

The characterization of changes in dominant processes was studied by the relationship between estimated sediment production and peak discharge. Different threshold values of peak discharge would indicate the change of sheet erosion to rill erosion, and modifications of the intensity in the rill process. This last point could be related to the loss of regulation capacity aforementioned. In those cases, there would be other factors of major importance in runoff and sediment production, such as mass and energy of the flow, the formation of preferential flow pathways and the connectivity of the landscape. All these factors would contribute to the change of the equilibrium, and to the high soil loss.

The highest differences between estimated sediment production by registered data and predicted values by the modified universal soil loss equation belonged to the events with the highest soil losses. These differences would show the complexity of sediment production 
Lluvia, escurrimiento y producción de sedimentos en una microcuenca agrícola del Sistema de Tandilia response in these events, and besides, the limitations of this model to predict soil loss in these cases. 


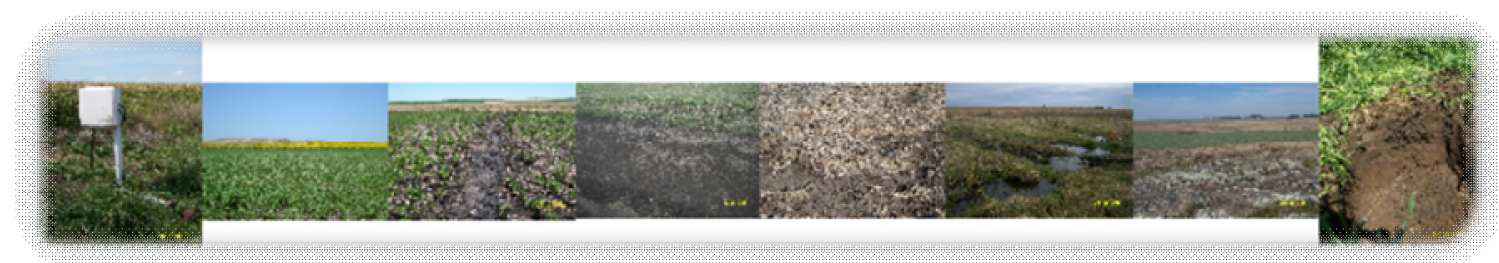

LLUVIA, ESCURRIMIENTO Y PRODUCCIÓN DE SEDIMENTOS EN UNA MICROCUENCA AGRÍCOLA DEL SISTEMA DE TANDILIA

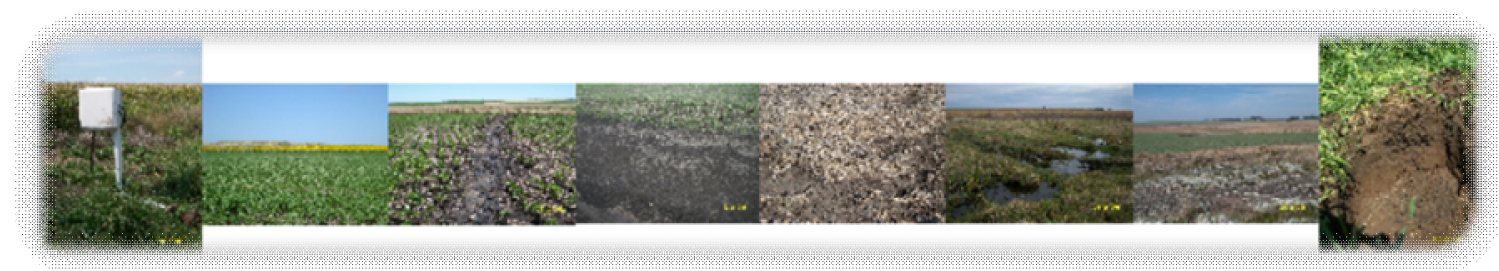




\section{INTRODUCCIÓN}

\subsection{Problema objeto de la Tesis}

La erosión hídrica es uno de los principales procesos de degradación que afectan a las tierras agrícolas a nivel mundial (Valentín et al., 2005; Pimentel et al., 1995). Sus efectos tienen consecuencias en el sitio donde ocurre, al disminuir la capacidad productiva de las tierras, ya que se ve afectada su calidad y como consecuencia de ello, los rendimientos de los cultivos (Lal, 2001; Duvert et al., 2010). Al mismo tiempo ocasionan externalidades, debido a la sedimentación en canales y reservorios y a la contaminación que puede provocar el incremento de patógenos o la concentración de nutrientes en las aguas, lo que afecta no sólo las actividades y la salud humana, sino también a la ecología de los ríos, arroyos y zonas costeras (Owens et al., 2005). La lluvia y el escurrimiento asociado son los principales desencadenantes de este proceso (Blanco \& Lal, 2008). La precipitación, a través del impacto que ejercen las gotas de lluvia es el principal agente de desprendimiento de las partículas de suelo de los agregados (Morgan, 2005), mientras que el flujo las moviliza, aunque también puede tener capacidad de desprendimiento cuando se concentra (Thornes, 1980; Terzoudi et al., 2007).

En la Argentina, estadísticas del año 1990 indican que existen 30 millones de hectáreas afectadas por erosión hídrica (Casas, 2000). Este proceso de degradación es de variada intensidad a lo largo del país, y se da mayormente en las provincias de Buenos Aires, Santa Fe, Córdoba, Entre Ríos, Misiones, Chaco, Formosa, mientras que de manera localizada en algunos sectores de Salta, San Juan, San Luis, La Rioja y en las provincias patagónicas de Santa Cruz y Chubut (Casas, 2000).

La siembra directa es una práctica de manejo que tiende a conservar el suelo y el agua, y constituye una alternativa para reducir el escurrimiento y la erosión en las tierras cultivables (Armand et al., 2009). Su efecto benéfico se relaciona con el incremento del contenido de carbono orgánico y con la protección edáfica que se producen al conservar los residuos de cosecha en superficie, lo que mejora la estabilidad de los agregados y propiedades del suelo como la macroporosidad, la retención de agua, la infiltración y la conductividad hidráulica (Zhang et al., 2007; Morris et al., 2010, Jin et al., 2011; Van Wie et al., 2013). Su incorporación produjo un cambio tecnológico que ha modificado la actividad agrícola en países como Estados Unidos, Brasil, Argentina y Canadá (Blanco \& Lal, 2008). En Argentina, su adopción se produjo rápidamente junto con la introducción de cultivos 
transgénicos y el aumento en el uso de agroquímicos en un proceso de incremento de la superficie destinada a la agricultura (agriculturización) que se vio favorecido por la mejora en los precios de los commodities y el incremento de las precipitaciones desde la mitad de la década de los años 70 (Manuel-Navarrete et al., 2009). Sin embargo, esta práctica no ha sido implementada de manera integral, con estrategias específicas de conservación de suelos que permitan el aprovechamiento del agua pluvial, lo que sumado al aumento de los niveles de compactación superficial, ha acelerado el escurrimiento superficial y facilitado los procesos erosivos (Cisneros et al., 2012).

En la región de las sierras de Tandilia, los cambios antes mencionados tuvieron como consecuencia la disminución de la superficie de pasturas naturales e implantadas y la intensificación de la actividad agrícola. De acuerdo con Sfeir et al. (2006), y en coincidencia con lo señalado, el sistema de siembra directa se introdujo de manera poco sustentable, asociado al uso de la sembradora de siembra directa combinada con laboreos previos, el pastoreo excesivo de los rastrojos y la elaboración de reservas forrajeras a partir de dichos residuos. Por lo tanto, no se ha tenido en cuenta la necesidad de cobertura y reposición de carbono orgánico y nutrientes que dicho sistema promueve. Además, las siembras o los laboreos con frecuencia no se realizan siguiendo las curvas de nivel, a pesar de que el área tiene relieve ondulado con pendientes entre el 3 y el 11\%, mientras que la planificación de las rotaciones es escasa, con alta participación del cultivo de soja, que ha registrado un incremento de superficie sembrada cercano al 25\% entre los años 2001 y 2011 en esta región (Holzman, 2013). Los rastrojos de este cultivo, son de bajo volumen y además ricos en nitrógeno, por lo que su descomposición es rápida y la incorporación de materia orgánica es muy escasa, además de poseer un sistema de raíces que generan menos bioporos en comparación con los que producen las raíces de las gramíneas (Casas, 2006). De este modo, los cambios ocurridos han provocado la reducción en la cobertura vegetal y en el efecto protector que ejerce. Esto conduciría a la modificación de las propiedades de los suelos, alterándose la porosidad, la capacidad de retención de agua y la estabilidad de los agregados del suelo, todo lo cual determinaría la reducción de la calidad estructural (FAO, 2000) lo que hace susceptible al suelo a la erosión hídrica.

Al mismo tiempo, el aumento de las precipitaciones registrado en las últimas décadas, en el contexto antes mencionado, han contribuido a incrementar el escurrimiento, y a favorecer las condiciones para la pérdida de suelo. Esta tendencia se evidencia por los desbordes recurrentes de los arroyos de la región, entre los cuales, el arroyo del Azul ha registrado este tipo de crecidas en los años 1980, 1985, 1992, 2001, 2002 y 2012, las que han ocasionado efectos adversos en el ámbito rural y urbano. La sedimentación aguas abajo 
posterior a estos eventos pone en evidencia la magnitud de la erosión sufrida. Sin bien las inundaciones son eventos extremos que se perciben claramente a nivel urbano, los daños por erosión hídrica en los lotes de producción también pueden ser advertidos por los propios productores ante la aparición de surcos y cárcavas en los mismos. De acuerdo con lo planteado, el proceso de degradación de erosión hídrica constituye un problema prioritario en la región de las sierras de Tandilia (Irurtia et al., 1996).

\subsection{Antecedentes existentes}

La comprensión del proceso de erosión hídrica así como la cuantificación de su magnitud, resultan fundamentales para la implementación de estrategias para su control (Blanco \& Lal, 2008). Ello requiere conocer los factores, procesos y mecanismos que intervienen en la erosión.

Los principales factores que controlan la erosión son la precipitación, la cobertura vegetal, el relieve y las propiedades de los suelos. En general, la cantidad de lluvia, su intensidad, y frecuencia determinan la magnitud de la erosión, mientras que la cobertura permite interceptar, adsorber y reducir parte de la energía de las gotas de lluvia. El relieve determina la velocidad del flujo de escurrimiento. En cuanto al suelo, las propiedades que condicionan la infiltración tales como el contenido de materia orgánica, textura y macroporosidad, inciden también en su erodabilidad (Blanco \& Lal, 2008)

De acuerdo con Morgan (2005), la erosión hídrica es un fenómeno que consta de tres fases: desprendimiento, transporte y sedimentación. Las gotas de lluvia, que generan impacto en la superficie del suelo descubierto, son el agente de desprendimiento más importante. El desprendimiento también puede ser realizado a través de flujos de escurrimiento concentrado de alta energía que pueden originar surcos y eventualmente cárcavas. Los agentes de transporte comprenden a aquellos que actúan a nivel de superficie y que pueden ayudar a remover un espesor relativamente uniforme de suelo y además, a los que ejercen su acción en canales. Los primeros incluyen a la salpicadura de las gotas de lluvia y al flujo superficial, mientras que los segundos están asociados a surcos o estructuras permanentes como las cárcavas. La sedimentación de las partículas puede ocurrir a cortas distancias o pueden ser arrastradas a puntos alejados del sitio donde fueron desprendidas, es decir, que puede producirse en cualquier punto de un suelo. 
Asociados a estas tres fases del proceso erosivo, pueden ser identificados mecanismos de erosión entre surcos y en surcos (Nearing et al., 1994). El primero de ellos se refiere al desprendimiento de las partículas y el transporte, mientras que el segundo se produce al concentrarse el escurrimiento en pequeños canales (Brady \& Weil, 2008). La erosión entre surcos se considera que puede ser relativamente más importante que la erosión en surcos en algunos casos particulares, como por ejemplo en sectores con pendientes cortas, en tierras de pastizal y en áreas bajo siembra directa (Nearing et al., 1994). West et al. (1992) establecen que las modificaciones que el sistema de siembra directa introduce en el horizonte superficial del suelo vinculado a la cobertura y a la no remoción contribuyen a las reducciones en la pérdida de suelo por erosión en surcos.

La caracterización del proceso en la región de las sierras de Tandilia requiere conocer los factores que condicionan las relaciones entre precipitación- escurrimiento-producción de sedimentos. En tal sentido, en el área de la cuenca del arroyo del Azul se han realizado diferentes estudios acerca de las precipitaciones de la zona (Entraigas et al., 2001; Varela et al., 2002) como así también, de la respuesta hidrológica de cuencas aforadas (Ares, 2010). Asimismo, se ha analizado la incidencia del uso y el manejo del suelo en la generación de escurrimiento a partir de lluvia simulada (Sfeir et al., 2005; Sfeir et al., 2006) y se ha caracterizado la potencialidad de escurrimiento en el área de estudio (Ares et al., 2005; Ares et al., 2012b). Paralelamente, Varni et al. (1996); Cazenave (2006) y Dalponte et al. (2007) implementaron modelos que simulan el proceso precipitación-generación de escurrimiento, los que resultan fundamentales en la predicción de la magnitud de las crecidas.

En cuanto al proceso de erosión, se han analizado la erosividad de las precipitaciones de la zona (Ares et al., 2010) y la erodabilidad de suelos de la cuenca alta del arroyo del Azul (Piscitelli et al., 2010). Sin embargo, aún no existen estudios básicos acerca de la generación de sedimentos que permitan evaluar la magnitud y las características del proceso de erosión hídrica en esta zona en la que predomina el sistema de siembra directa. Resulta fundamental el análisis de la interacción precipitación-escurrimiento-producción de sedimentos que permita identificar distintos tipos de respuesta, para poder así elaborar propuestas de manejo para el control de la erosión acordes a la intensidad y características de este proceso en la región.

De acuerdo con Mutchler et al. (1994), la investigación de dicho proceso puede basarse en resultados experimentales obtenidos en parcelas en laboratorio bajo lluvia simulada, o en parcelas a campo, también con lluvia simulada o bajo precipitación natural. Las parcelas pequeñas permiten estudiar en detalle aspectos básicos de la erosión: desprendimiento por 
parte de las gotas de lluvia, transporte por salpicadura y el sellado edáfico superficial, los cuales están vinculados a la erosión laminar, mientras que las parcelas de mayor tamaño dan información acerca del proceso combinado de la erosión laminar y en surcos.

Sin embargo, son necesarios estudios a escala de cuenca para poder realizar el análisis integrado del escurrimiento, pérdida de suelo, su transporte y depositación. Esto es así dado que la cuenca es la unidad en la que se expresan los procesos hidrológicos condicionados, entre otros, por aspectos geomorfológicos (Blöschl \& Sivapalan, 1995). Así, representa la unidad significativa sobre la cual pueden ser implementadas tanto estudios básicos sobre procesos degradatorios como también medidas para su control y manejo (Cerdan et al., 2004, Collins y Owens, 2006). Es por ello que varios autores han analizado a nivel de cuenca la variabilidad en las relaciones entre la lluvia, el escurrimiento y la producción de sedimentos, asociadas a condiciones de humedad antecedente, uso de suelo y sistemas de labranza implementados (Shipitalo \& Edwards, 1998; Steegen et al. 2000; Schmidt \& Morche, 2006; Zabaleta et al., 2007; García-Ruiz et al., 2008; Nadal-Romero et al., 2008; Bilotta et al., 2010; Polyakov et al., 2010; Rodríguez-Blanco et al., 2010; Onderka et al., 2012; Gellis, 2013). Asimismo, han sido investigadas las relaciones entre la concentración de sedimentos y la descarga de agua de las crecidas para eventos hidrológicos individuales (Williams, 1989; Lenzi \& Marchi, 2000; Nu-Fang et al., 2011), lo que da origen a patrones de histéresis. El análisis de estas relaciones contribuye a interpretar los procesos de entrega y distribución de sedimentos en las cuencas, como también a identificar la distribución espacial de las áreas fuente de los sedimentos (Duvert et al., 2010; Oeurng, 2010).

Por otra parte, los modelos matemáticos constituyen herramientas útiles para comprender los procesos de erosión y su impacto en un área determinada. Existen 3 tipos de modelos según los procesos físicos que simulan, los algoritmos que describen dichos procesos y la dependencia de datos por parte del modelo (Merrit et al., 2003). Esos tipos son los empíricos, los conceptuales y los basados en procesos físicos. Su elección depende de la información de salida deseada y de los datos disponibles para el área de estudio en la cual se lo implementará (Maalim et al., 2013). Los modelos empíricos son los más simples, pero suelen ser cuestionados por ignorar la heterogeneidad de las entradas a la cuenca o sus características, como la precipitación o los tipos de suelos. Si bien esto resulta cierto, la escasa información meteorológica existente o en cuanto a los tipos de suelos, dificulta la implementación de modelos más complejos (Merrit et al., 2003).

Dentro de los procedimientos empíricos, la denominada "Ecuación Universal de Pérdida de Suelo" (USLE) (Wischmeier y Smith, 1978) es el modelo de predicción más ampliamente 
utilizado debido a su facilidad de aplicación (Kinnel, 2007). Dicha ecuación fue desarrollada con el objetivo de orientar la toma de decisiones en la planificación de conservación de suelos, ya que permite predecir la tasa promedio de erosión para varias combinaciones alternativas de sistemas de cultivos, técnicas de manejo y prácticas de control en diferentes sitios. El valor de pérdida de suelo resultante comparado con aquella que es tolerable para el área que se considere, da las pautas para implementar las prácticas de control de erosión. Es decir que las combinaciones de uso y manejo para las cuales el nivel de erosión predicho esté por debajo del valor tolerable serán satisfactorias para el control de este proceso de degradación (Wischmeier \& Smith, 1978). De igual modo, ha sido utilizada en el mundo con el objetivo de determinar los valores numéricos de los diferentes componentes del proceso erosivo (da Silva, 2004) y para estimar la pérdida media anual de suelo a largo plazo asociada con la erosión laminar y en surcos (Kinnell, 2010). Así, la información que proporciona permite identificar áreas con riesgo de erosión, lo que resulta fundamental para seleccionar medidas de conservación como también para orientar la planificación del uso de la tierra (Gabriels et al., 2003).

Este modelo estima la erosión como la combinación de 5 factores principales (Wischmeier \& Smith, 1978):

$$
A=R * K^{*} L^{*} S * C * P
$$

Donde $A=$ Pérdida de suelo calculada ( $(\text { (ha año })^{-1}$ )

$\mathrm{R}=$ Factor de erosividad de las precipitaciones ( $\mathrm{MJ} \mathrm{mm}$ (ha $\mathrm{h}$ año) ${ }^{-1}$ )

$\mathrm{K}=\mathrm{Factor}$ de erodabilidad del suelo ( $\mathrm{T}$ ha $\mathrm{h}$ (ha $\mathrm{MJ} \mathrm{mm})^{-1}$ )

$\mathrm{L}=$ Factor de longitud de la pendiente

$\mathrm{S}=$ Factor de inclinación de la pendiente

$\mathrm{C}=$ Factor de cobertura y manejo

$\mathrm{P}=$ Factor de prácticas de manejo

Sin embargo, la USLE no hace consideración directa de la escorrentía, si bien la erosión depende del sedimento que se descarga con el flujo y varía con el escurrimiento y la concentración del material transportado (Kinnell, 2005). En este sentido, una variante del modelo de la USLE es el denominado MUSLE (Ecuación Universal de Pérdida de Suelo Modificada), en el que el factor de la erosividad de la lluvia es reemplazado por un factor dependiente del escurrimiento (Williams \& Berndt, 1977), mientras que el resto de la ecuación se mantiene igual que la correspondiente a la USLE: 


$$
Y=11.8(Q q p)^{0.56} * K * L * S * C * P
$$

Donde $\mathrm{Y}=$ Rendimiento de sedimento en la cuenca $(\mathrm{T})$

$\mathrm{Q}=$ escurrimiento superficial $\left(\mathrm{m}^{3}\right)$

$\mathrm{qp}=$ caudal pico $\left(\mathrm{m}^{3} \mathrm{~s}^{-1}\right)$

En esta modificación de la USLE, el factor de escorrentía representa la energía para los procesos de desprendimiento, asociados al escurrimiento superficial, y el transporte de las partículas, relacionado con el caudal pico (Williams \& Berndt, 1977). El modelo fue desarrollado a partir de datos de pequeñas cuencas de Estados Unidos, para las cuales explicó más del 90\% de la variación en la producción de sedimentos (Williams, 1975). Así, además de ser aplicado a escala de cuenca, permite estimar la producción de sedimentos en tormentas individuales (Williams \& Arnold, 1997; Kinnell, 2010). El mismo ha sido utilizado por distintos investigadores en el mundo (Jackson et al., 1986; Sadeghi \& Mizuyama, 2007; Pandey et al., 2009) como en nuestro país (Gaspari, 2005; Marizza et al., 2010, Besteiro \& Gaspari, 2012). Por otra parte, la ecuación que propone MUSLE se encuentra incluida en modelos que evalúan el efecto de estrategias de manejo en la producción agrícola y en los recursos agua y suelo a nivel de cuenca tales como EPIC y SWAT (Williams \& Arnold, 1997; Nearing et al., 2005).

\subsection{Objetivos}

\section{Objetivo general}

Estudiar el proceso erosión hídrica en una microcuenca ubicada en la subcuenca superior del arroyo del Azul, que se encuentra en el Sistema de Tandilia, por medio de la evaluación de la precipitación, el escurrimiento y el transporte de sedimentos en un curso de agua, como también a través de la aplicación de un modelo empírico que describa dicho proceso.

\section{Objetivos específicos}

-Realizar mediciones de los sedimentos que son transportados en un curso de agua que descarga una microcuenca de monitoreo.

-Analizar las relaciones entre las variables involucradas en el proceso lluvia-escurrimientoproducción de sedimentos. 
-Calibrar un modelo de uso internacional para la estimación de erosión hídrica a nivel de microcuenca.

-Comparar los resultados obtenidos a través de las distintas metodologías de análisis aplicadas.

\subsection{Hipótesis}

- En el área bajo estudio existe relación entre las características de las tormentas, generación de escurrimiento superficial y producción de sedimentos

- El escurrimiento y la producción de sedimentos sufren modificaciones a lo largo del año en función de la estacionalidad de las tormentas y de la variación en el uso del suelo

- El proceso de erosión laminar resulta predominante frente al de erosión en surcos 


\section{MATERIALES Y MÉTODOS}

\subsection{Descripción del área de estudio}

El área de estudio corresponde a una microcuenca ubicada en la cuenca superior del arroyo del Azul, que está situado en el centro de la provincia de Buenos Aires (Figura 2.1.a) y b)). Sobre las márgenes de dicho arroyo se sitúa la ciudad de Azul, capital del Partido correspondiente, con 58097 habitantes (INDEC, 2010). La microcuenca cuenta con una extensión de 566 ha, se encuentra en las nacientes del arroyo Videla (tributario del arroyo del Azul) y las coordenadas de su punto de cierre son $37^{\circ} 08^{\prime} 48^{\prime \prime} \mathrm{W}$; $59^{\circ} 55^{\prime} 25^{\prime \prime} \mathrm{S}$.

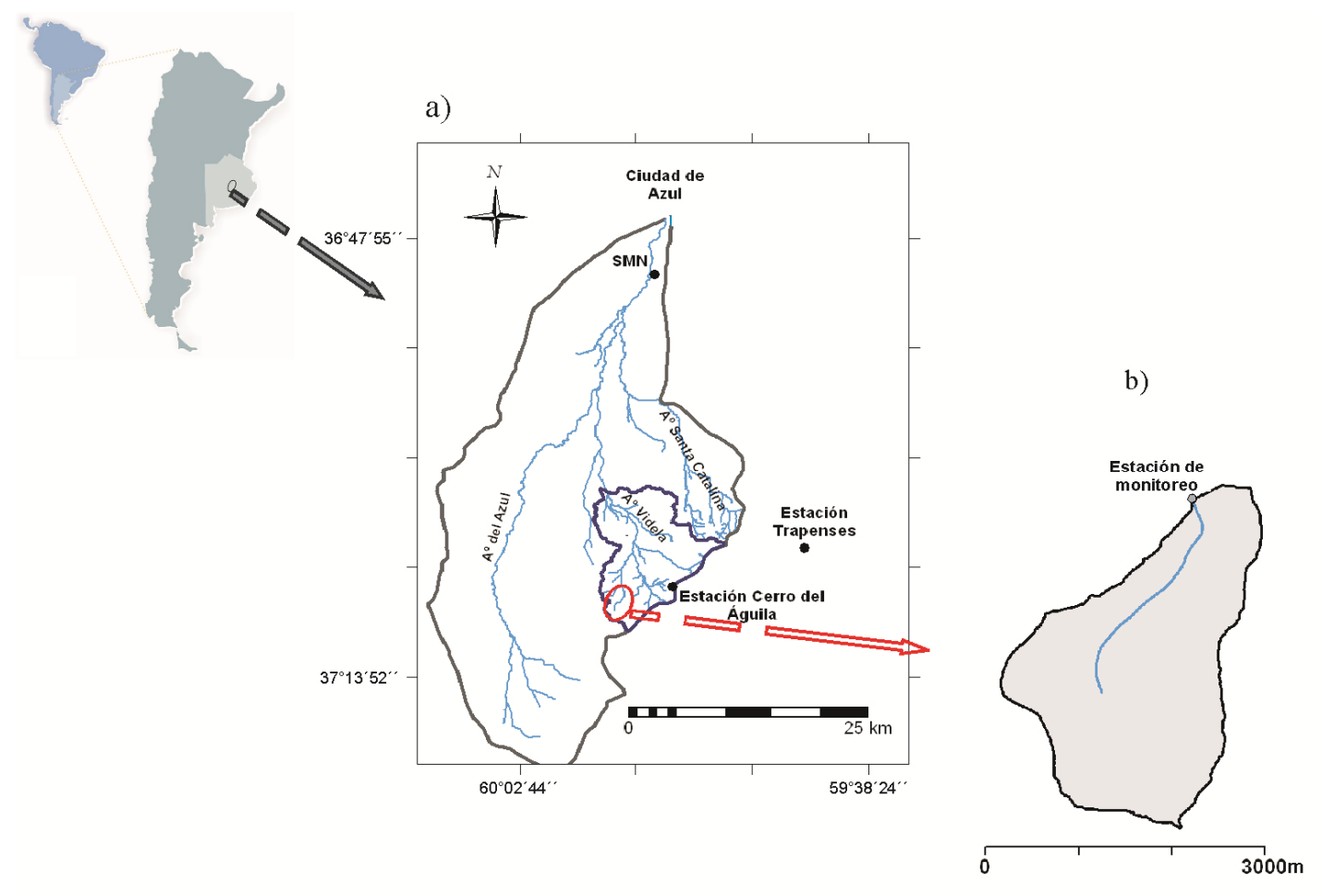

Figura 2.1.a) Ubicación de la microcuenca en estudio en la cuenca superior del arroyo del Azul, estaciones de registros pluviométricos (Trapenses y Cerro del Águila) y meteorológicos

(Servicio Meteorológico Nacional, SMN). b) Detalle de la microcuenca y ubicación de estación de monitoreo de caudales y muestreo de agua de crecidas.

Las características climáticas pueden ser descriptas a través de la información suministrada por la estación Azul Aero del Servicio Meteorológico Nacional, que es la más cercana al área de estudio que cuenta con registros históricos. Para el período 1901-2012 la 
precipitación media anual es de $914 \mathrm{~mm}$. La temperatura media es de $14,5{ }^{\circ} \mathrm{C}$ (período 1966-2003), con temperatura máxima media mensual de enero de $21,4^{\circ} \mathrm{C}$ y temperatura mínima media mensual de julio de 7,7ํㅡ. Según la clasificación climática de Thornthwaite, el clima de la región se clasifica como subhúmedo-húmedo, mesotermal, con poca o nula deficiencia de agua (Entraigas et al., 2012).

La distribución de las lluvias en el año (Figura 2.2), analizada a través las medias mensuales, indica que las precipitaciones se concentran principalmente entre enero y abril y entre octubre y diciembre. Marzo es el mes más lluvioso $(117,6 \mathrm{~mm})$ y julio el menos lluvioso $(45,1 \mathrm{~mm})$.

Por otra parte, Ares et al. (2010) analizaron la erosividad de las precipitaciones según la metodología propuesta por Wischmeier y Smith (1978) para un período de registros pluviográficos disponibles, comprendido entre 1995 y 2007. Así, determinaron un valor promedio anual del factor $\mathrm{R}$ de $2486,8 \mathrm{MJ} \mathrm{mm}$ (ha $\mathrm{h}$ año) ${ }^{-1}$, mientras que la distribución mensual del índice (Figura 2.3) muestra que los mayores valores corresponden a febrero, marzo y abril, y los menores a los meses invernales. De este modo la distribución de la erosividad resulta acorde a la que presentan las precipitaciones en la zona.

La geomorfología de la microcuenca es la del sistema de sierras de Tandilia, que está constituido por una cadena de cerros aislados de rocas graníticas (de edad antiguaPrecámbrico y Paleozoico-), áreas de pedemonte y lomas con relieve ondulado. La superficie que comprende la microcuenca posee altitudes máximas entre 260 y 280 metros sobre el nivel del mar (msnm). Los sectores rocosos ocupan áreas reducidas con respecto a los pedemontes serranos donde se desarrollan los suelos a partir de loess depositado en un espesor que oscila entre 1 y 2 metros sobre una costra calcárea de gran dureza (denominada localmente como "tosca"), que suele aflorar en las lomas (INTA-CIRN, 1990). La pendiente media de la microcuenca es de $3 \%$, con sectores que pueden superar el $10 \%$ de pendiente y otros en los que puede ser menor que $1 \%$.

De acuerdo con lo descripto y con la cartografía disponible (INTA, 1992), en la microcuenca predominan los Argiudoles típicos (67,9\%) con buen drenaje, horizonte superficial de textura franco arcillosa con contenidos de carbono orgánico (CO) entre 3 y $4 \%$. En menor proporción, en las áreas de cerros y lomas con roca o tosca cercana a la superficie se encuentran Hapludoles y Argiudoles líticos (27,6\%). Sus propiedades pueden ser variables, y se han relevado aquellos cubiertos por pasturas naturales que poseen un horizonte superficial con alta proporción de raíces, alto contenido de CO $(6,1 \%)$ y estructura granular. 
Asimismo, las lomas de uso agrícola, con mayor espesor del horizonte $A$, presentan contenidos de $\mathrm{CO}$ cercanos a $3 \%$ y estructuras en bloques. Por último, el $4,5 \%$ de la superficie corresponde a los suelos con menor capacidad de drenaje, cercanos al cauce principal de la microcuenca. En general, los suelos de la microcuenca presentan estructuras estables y porosas.

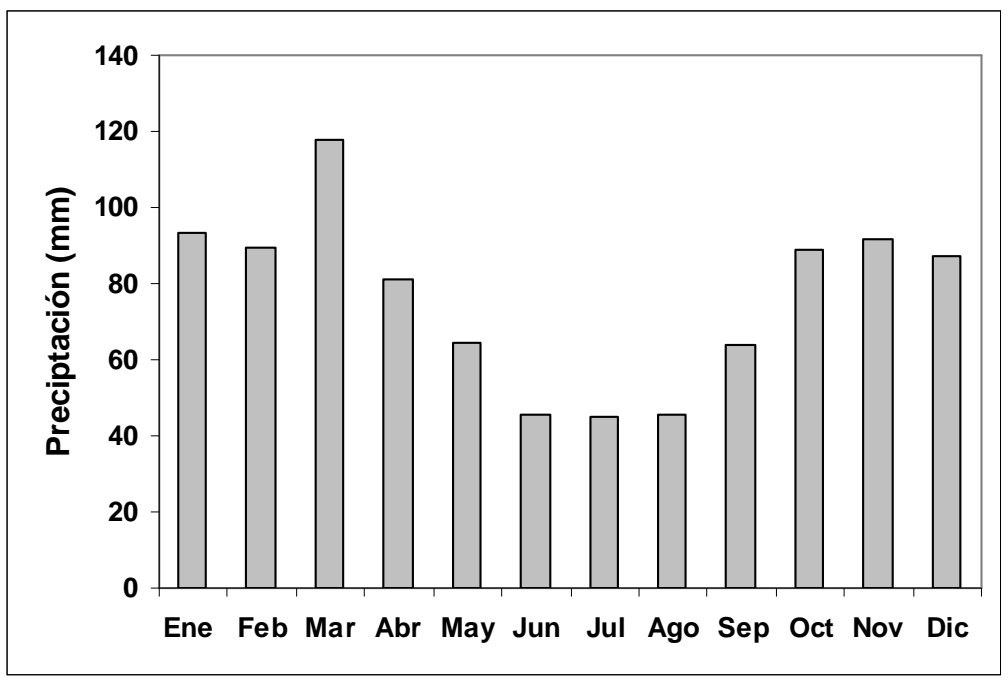

Figura 2.2. Precipitaciones medias mensuales correspondientes al período 1901-2012, registradas en la estación Azul Aero del Servicio Meteorológico Nacional.

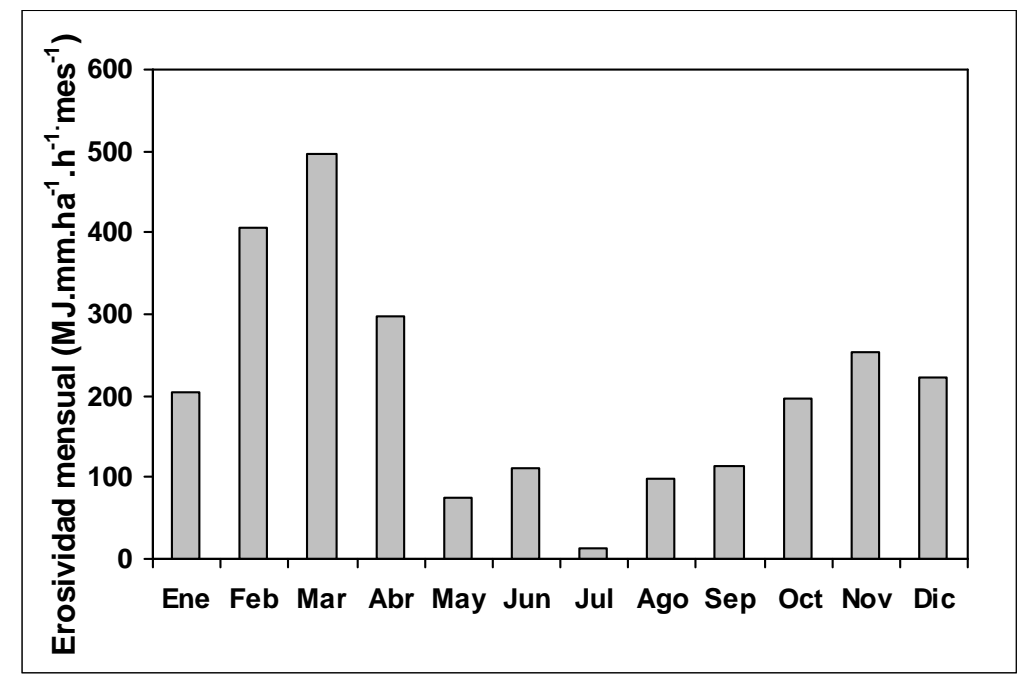

Figura 2.3. Erosividad mensual de las precipitaciones de la localidad de Azul, calculadas según la metodología propuesta por Wischmeier y Smith (1978).

La superficie de la microcuenca corresponde a 3 establecimientos agropecuarios, y los suelos de los lotes que comprenden el área considerada se destinan a actividades agrícolas. 
Allí se implementan rotaciones que incluyen a los cultivos de trigo, cebada, soja, maíz o girasol bajo sistema de siembra directa, mientras que no se encuentran estructuras de conservación de suelos permanentes tales como terrazas o canales interceptores.

Para realizar el análisis planteado en este trabajo de tesis se consideraron datos de precipitación, escurrimiento, condiciones antecedentes y producción de sedimentos de las tormentas registradas durante 2011 y 2012 en el área bajo estudio. A continuación se describen los métodos empleados para la obtención y cálculo de esta información.

\subsection{Precipitaciones y variables relacionadas: registro, cálculos y caracterización para el área de estudio}

\subsubsection{Precipitaciones}

La lluvia se midió por medio de una estación meteorológica automática perteneciente a un sistema de alerta contra inundaciones de la ciudad de Azul, Argentina. Dicha estación denominada "Cerro del Águila" se encuentra situada a $5 \mathrm{~km}$ del punto de cierre de la microcuenca en estudio (Figuras 2.1 y 2.4) y es la más cercana a la microcuenca que cuenta con datos detallados para el período analizado. La misma posee un pluviómetro construido y dispuesto según las normas de la Organización Meteorológica Mundial, que registra la lluvia cada 10 minutos con una precisión de $0,20 \mathrm{~mm}$ a través de un sistema de cangilones. Se calculó el valor total de la precipitación ( $\mathrm{P}, \mathrm{mm})$, y su duración (Dur, h).

Se analizó la distribución mensual de las precipitaciones en el área de estudio, a través de datos históricos para la zona, con el objetivo de establecer comparaciones con la distribución correspondiente a los años considerados en este trabajo. Dado que no se contaba con este tipo de registros para la estación Cerro del Águila, se emplearon aquellos pertenecientes a la Estación Monasterio Trapense, ubicada aproximadamente a $20 \mathrm{~km}$ del punto de cierre de la microcuenca (Figura 2.1), y cuya información resulta confiable (Varni \& Custodio, 2013a). Se realizó un análisis de dobles masas para comparar las lluvias mensuales de las estaciones Cerro del Águila y Trapenses, durante el período de datos en común, los años 2011 y 2012. 


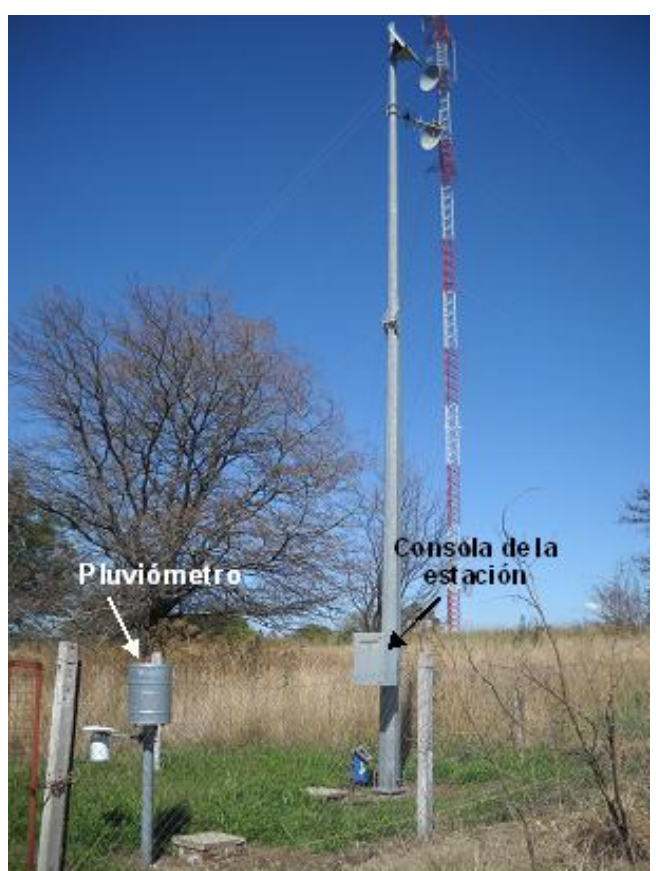

Figura 2.4. Estación meteorológica "Cerro del Águila".

\subsubsection{Energía de la precipitación, intensidades máximas en 10 y 30 minutos e índice $\mathrm{El}_{30}$}

A partir de los datos de precipitación de 10 minutos, se calculó la energía cinética total de la precipitación $(E, M J$ ha-1 $)$, la intensidad máxima en 10 y en 30 minutos $\left(l_{10}\right.$ e $I_{30}$ respectivamente, $\left.\mathrm{mm} \mathrm{h}^{-1}\right)$, y el producto $\mathrm{El}_{30}\left(\mathrm{MJ} \mathrm{mm}(\mathrm{ha} \mathrm{h})^{-1}\right)$. La $\mathrm{E}$ de la precipitación se obtuvo a partir de la sumatoria de las energías individuales de intervalos de 10 minutos según la relación matemática establecida por Wischmeier \& Smith (1978)

$$
e=0,119+0,0873 \log _{10}(i)
$$

donde $\mathrm{e}=$ energía cinética del intervalo, en $\left(\mathrm{MJ}(\text { ha } \mathrm{mm})^{-1}\right)$, e

$\mathrm{i}=$ intensidad de la lluvia, en $\mathrm{mm} \mathrm{h-1.}$

Por su parte, la intensidad máxima en 30 minutos se estimó considerando la máxima precipitación caída en intervalos variables de 30 minutos.

Asimismo, se analizó la distribución mensual del $\mathrm{El}_{30}$ para el período estudiado. Para ello, se adoptó la definición de tormenta individual establecida por Wischmeier (1959), que considera como tal a las lluvias separadas por menos de 6 horas, mientras que precipitaciones menores que $12,7 \mathrm{~mm}$ fueron omitidas de los cálculos, por ser de baja 
magnitud (Renard et al., 1997). Se calcularon los valores individuales de las tormentas, los $\mathrm{El}_{30}$ mensuales (sumando los $\mathrm{El}_{30}$ individuales de cada mes) y finalmente el valor total anual de esta variable. Los valores obtenidos se compararon con los estimados por Ares et al. (2010) para la localidad de Azul (Figura 2.1) mediante registros pluviográficos de 13 años, de manera de analizar las diferencias entre los años estudiados y datos de una serie más larga que considere patrones cíclicos de las lluvias.

\subsection{Condiciones antecedentes de humedad}

\subsubsection{Reserva de agua en el suelo}

Se calcularon valores locales de reserva de agua en el suelo para evaluar las condiciones antecedentes de humedad. Este valor demostró ser un buen estimador de la humedad previa en predicciones del escurrimiento realizadas en la subcuenca del arroyo Videla, donde se encuentra ubicada el área de estudio del presente trabajo (Ares et al., 2012a).

La reserva de agua en el suelo se calculó a partir de un balance hídrico de paso diario cuyo procedimiento es el que a continuación se describe. Los parámetros del balance son la capacidad de campo $(\mathrm{mm})$, el punto de marchitez permanente $(\mathrm{mm})$, la profundidad de exploración radical $(\mathrm{m})$, la reserva de agua inicial $(\mathrm{mm})$ y la capacidad de infiltración máxima diaria $(\mathrm{mm})$. Si la lluvia diaria excede este último valor, dicho exceso se cuantifica como escurrimiento superficial. La variación de reserva diaria se calcula como el agua infiltrada (la lluvia o la capacidad de infiltración máxima) menos la evapotranspiración de referencia (ETr) diaria. A continuación se verifica que la variación de reserva del día i más la reserva útil del día i-1 no sea mayor que la capacidad máxima de la reserva útil (diferencia entre capacidad de campo y punto de marchitez permanente, por el espesor radical) ni menor que cero. Luego se calcula el déficit o exceso como la suma de la reserva útil del día i-1 más la variación de reserva del día i menos la reserva útil del día i. Finalmente, se calcula la evapotranspiración real del día i igual a la ETr en el caso de que haya habido exceso o igual a la ETr menos el déficit en el caso de que haya habido déficit. Debido a que se desconocía la humedad inicial para el 1 de enero de 2011, el balance se inició desde 2010, aunque hubiese sido más correcto su inicio en 2009 para luego descartar esos 2 años, ya que después de ese período, y a través de la mecánica del balance se alcanza al valor real de la reserva de agua edáfica (Pascale y Damario, 2004).

La evapotranspiración de referencia (ETr), se calculó mediante el método de PenmanMonteith recomendado por la FAO, cuya validez ha sido demostrada tanto para climas 
húmedos como secos (Allen et al., 1998) a partir de los datos registrados por la estación Azul Aero del Servicio Meteorológico Nacional. Si bien esta estación se encuentra a $35 \mathrm{~km}$ del punto de cierre de la microcuenca, se considera que la evapotranspiración resulta, en términos generales, relativamente homogénea en ambas zonas, y aún en estaciones más distanciadas tal como señalan Rivas \& Caselles $(2004,2005)$, razón que fundamenta el empleo de estos datos para su cálculo.

Se utilizaron los datos de constantes hídricas reportados por Weinzettel (2005) para suelos del área de estudio. Se fijó una capacidad máxima de reserva de agua en el suelo de 100 mm (Burgos \& Forte Lay, 1978), que está de acuerdo con las propiedades de los suelos presentes en el área de estudio (clase textural, contenido de materia orgánica, capacidad de almacenamiento en función de la profundidad efectiva de los distintos tipos de suelos presentes en la subcuenca). Asimismo, dicha capacidad máxima de reserva de agua establecida está asociada con la profundidad que ocupan las raíces más activas, que es la zona en la cual se evalúan los flujos de entrada y salida del agua en el cálculo del balance de agua en el suelo (Allen et al., 1998). En este caso, se estableció dicha profundidad a 0,6 m. Para cada evento estudiado se consideró entonces la reserva de agua en el suelo del día previo a la precipitación que dio origen al evento (ResA, mm) para evaluar las condiciones de humedad antecedentes. Asimismo, para realizar una caracterización del período estudiado se calculó la reserva media diaria de los años 2011 y 2012, como los valores medios diarios mensuales de esta variable.

\subsubsection{Precipitación de los 5 días previos}

Se consideró también a la precipitación de los 5 días previos como otra variable para evaluar las condiciones antecedentes en la microcuenca. Su valor fue calculado a partir de los registros de precipitación diaria correspondientes a la estación "Cerro del Águila", cuyas características fueron detalladas en la sección 2.2.1.

\subsection{Variables hidrológicas: registro y cálculos realizados}

Para este estudio, se consideraron la lámina de escurrimiento superficial (Esc, mm), caudal pico (Qp, $\left.\mathrm{m}^{3} \mathrm{~s}^{-1}\right)$, caudal superficial medio $\left(\mathrm{Qsm}, \mathrm{m}^{3} \mathrm{~s}^{-1}\right.$ ) y coeficiente de escurrimiento (CE, $\%$ ), calculado a través de la relación entre el escurrimiento superficial y la precipitación total del evento, según la Ecuación (2.2): 


$$
C E=\frac{E s c}{\text { Ptot }} * 100
$$

Asimismo se calculó el tiempo al pico de la crecida (Tp), valorado como el tiempo transcurrido entre el inicio de la crecida y el momento en que se alcanza el caudal pico. Estas variables se obtuvieron a partir de los datos de escurrimiento, que se registraron durante el período 2011-2012, con un limnígrafo con sensor de presión ubicado en la estación de monitoreo instalada a la salida de la microcuenca (Figuras 2.1 y 2.5). El nivel de agua se midió cada 15 minutos y se transformó en caudal a través de la curva altura-caudal de la sección obtenida a través de aforos realizados con molinete hidrométrico. Para este estudio se consideraron las crecidas para las cuales se obtuvieron muestras de agua de escurrimiento superficial, que fueron aquellas que igualaron o superaron los $0,3 \mathrm{~m}$ de altura, según se detalla en el próximo punto.

La separación del escurrimiento superficial del subterráneo se realizó mediante la aplicación de un filtro digital (Rodríguez et al., 2000), basado en uno de los métodos que revisa Chapman (1999). El principio sobre el cual se sustenta el filtro digital consiste en remover la componente de alta frecuencia del hidrograma de caudales, esto es la escorrentía directa, y así determinar la escorrentía de baja frecuencia: el flujo base.

a)

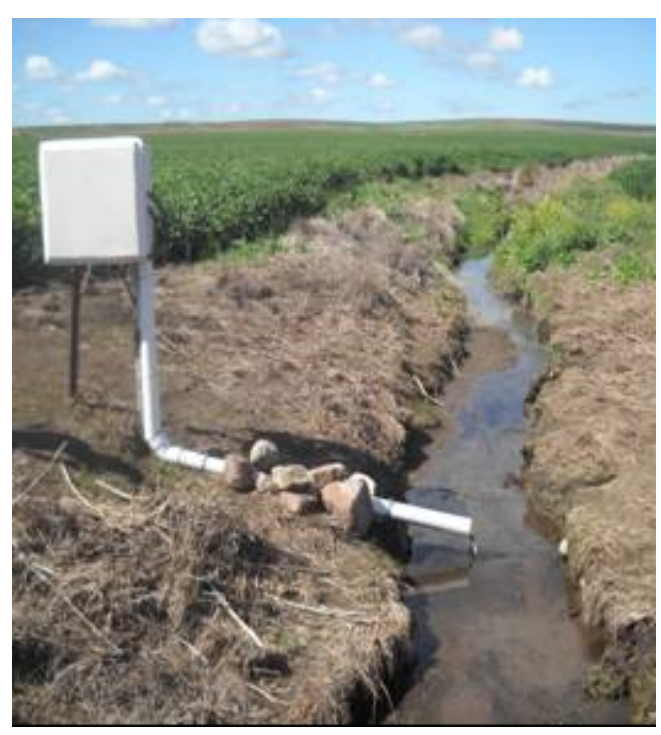

b)

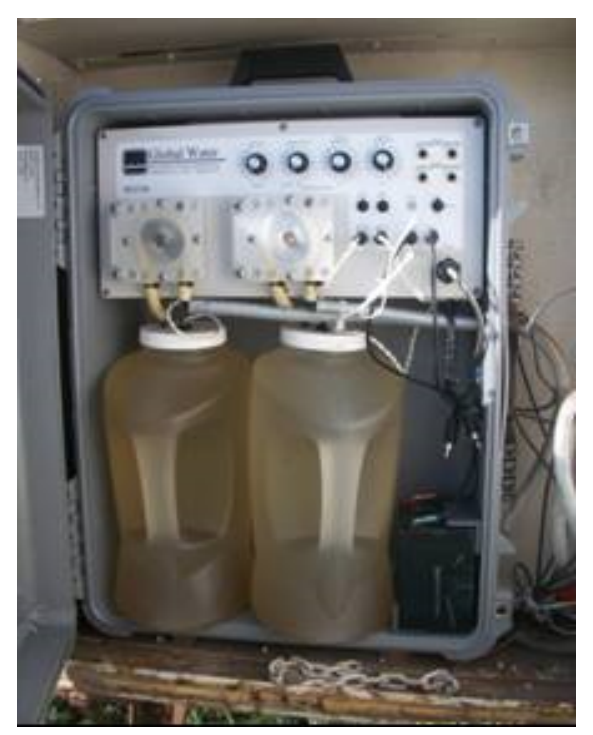

Figura 2.5. a) Estación de monitoreo de alturas y de agua de escurrimiento. b) Detalle de muestreador automático de agua y sedimentos. 
Otra variable hidrológica que fue considerada es el Número de Curva (USDA SCS, 1985). El método del Número de Curva (CN) expresa el volumen de agua disponible para escurrir superficialmente como una función de la lluvia y el almacenamiento (Silveira et al., 2000), según la Ecuación (2.3)

$$
Q=\frac{(P-0.2 S)^{2}}{P+0.8 S} \text { para } P>0.2 S
$$

mientras que $Q=0$ para $P \leq 0.2 S$, donde: $Q=$ escorrentía $(\mathrm{mm}) ; P=$ precipitación $(\mathrm{mm}) ; S=$ retención potencial $(\mathrm{mm})$.

En este caso, $S$ es el único parámetro que caracteriza el comportamiento del escurrimiento en un sitio y puede tomar valores desde cero a infinito. El Servicio de Conservación de Recursos Naturales (NRCS) de Estados Unidos concibió una transformación de $S$, en la que esta variable se expresa como el parámetro adimensional CN (Ecuación 2.4):

$$
C N=\frac{25400}{(254+S)}
$$

donde 25400 y 254 son constantes arbitrarias que se encuentran en las mismas unidades que $S(\mathrm{~mm})$. El $\mathrm{CN}$ varía en el rango $100 \geq \mathrm{CN} \geq 0$. Un valor de $\mathrm{CN}=100$ representa una condición de retención potencial nula $(S=0)$, esto es, una cuenca impermeable, en donde toda la lluvia se transforma en escorrentía. De manera inversa, $\mathrm{CN}=0$ representa una cuenca con capacidad de abstracción infinita $(S=\infty)$, o bien, sin escurrimiento (Hawkins, 1993; Ponce \& Hawkins, 1996).

Las principales características de la cuenca que afectan al parámetro $\mathrm{CN}$ son el tipo de suelo, la cobertura del suelo (que incluye el uso y el tratamiento o práctica), la condición hidrológica (referida al grado de cobertura), la condición de humedad antecedente y el clima de la cuenca. Estos factores principalmente determinan el potencial de infiltración de una cuenca. Es así que los valores de $\mathrm{CN}$ se encuentran tabulados para varios usos del suelo y prácticas, condición hidrológica y sus combinaciones, para cada tipo hidrológico de suelo (Mishra \& Singh, 2003).

Por otra parte, cuando se cuenta con datos locales de precipitación y escurrimiento es posible determinar los valores de $\mathrm{CN}$ correspondientes a los eventos registrados, a través de la expresión que se obtiene de la resolución de la Ecuación (2.3) 


$$
S=5\left[P+2 Q-\left(4 Q^{2}+5 P Q\right)^{1 / 2}\right]
$$

Entonces, cualquier par $(P, Q)(0<Q<P)$ da una solución para $S$ y mediante la Ecuación (2.4), un valor de CN (Hawkins, 1993).

De esta manera, se asociaron los pares lluvia-escorrentía registrados para cada evento y se procedió a calcular los valores de S a través de la Ecuación (2.5) y por último, los CN mediante la Ecuación (2.4).

\subsection{Muestreos de agua de escurrimiento durante las crecidas}

Los muestreos de agua de escorrentía durante las crecidas se realizaron a través de un muestreador automático instalado en la estación de monitoreo ubicada en el punto de cierre del área de estudio (Figuras 2.1 y 2.5). El dispositivo posee dos bombas recolectoras ubicadas dentro del cauce, distanciadas aproximadamente $10 \mathrm{~cm}$ desde el fondo, lo que permite la recolección de agua con material en suspensión. Las mismas fueron programadas para realizar dos tipos de muestreos: discreto y compuesto. $A$ través del muestreo discreto, el equipo toma una muestra completa de agua correspondiente a un punto en el tiempo, resultado de 7 minutos de recolección, de un volumen total de 3,8 litros. En el muestreo compuesto extrae una muestra de igual volumen que en el caso anterior pero constituida por submuestras distribuidas en el tiempo. Así permite obtener un valor de concentración promedio en el período de recolección, que se establece a través del intervalo de extracción y del volumen de la submuestra. Para este estudio se programó el equipo de manera tal que el muestreo se llevó a cabo durante 1,5 horas, con el objetivo de obtener datos de la evolución de la producción de sedimentos en un período más prolongado durante la crecida.

Además el muestreador posee dos sensores que activan el inicio de la recolección cuando toman contacto con el agua de la crecida transportada por el arroyo. Dichos sensores se ubicaron para que el muestreo se produzca cuando el nivel del curso de agua alcanza 0,3 $\mathrm{m}$ desde el fondo del cauce, es decir que ingresaron en el análisis las crecidas que igualaron o superaron ese nivel. El nivel se adoptó con el criterio de obtener muestras de agua de una cantidad razonable de eventos de magnitud significativa para esta microcuenca. 
Una vez en laboratorio, cada muestra se agitó, se tomó una alícuota de $250 \mathrm{~cm}^{3}$ y se secó en estufa a temperatura de 60ํㅡ hasta peso constante, según ASTM D3977-97 (2007). La determinación se realizó por duplicado. A partir de estos datos se calculó la concentración de sólidos suspendidos en la muestra (CSS disc y CSS comp), que se expresó en $\mathrm{g} \mathrm{I}^{-1}$.

\subsubsection{Producción de sedimentos estimada}

A partir de los datos de concentración del muestreo compuesto, y de acuerdo con el volumen de escurrimiento superficial total de los eventos, se estimó un valor de producción de sedimentos (ProdSedEst) probable para las crecidas analizadas. El objetivo de este cálculo consistió en obtener valores de la producción de sedimentos que podría haber sido generada en las crecidas, para establecer un análisis comparativo con los resultados de aplicación del modelo MUSLE.

\subsection{Parámetros de la Ecuación Universal de Pérdida de Suelo Modificada}

Se calcularon los parámetros correspondientes a la Ecuación Universal de Pérdida de Suelo Modificada según los procedimientos detallados a continuación.

\subsubsection{Factor de escurrimiento}

Este factor, como ya se mencionó, evalúa la energía del escurrimiento, y se calculó para cada evento analizado a través de la expresión propuesta por Williams \& Berndt (1977), presente en la Ecuación (1.2).

Para ello se emplearon los datos resultantes del procesamiento de las alturas registradas a la salida de la microcuenca, descriptos en la sección 2.4, el escurrimiento y el caudal pico correspondientes a cada evento estudiado.

\subsubsection{Factor de erodabilidad del suelo (K)}

El factor $\mathrm{K}$ considera la susceptibilidad del suelo a erosionarse asociada a propiedades como la textura, contenido de carbono orgánico, el tipo de estructura y la permeabilidad 
(Wischmeier et al., 1971), y en este caso se calculó a través de la ecuación propuesta por Wischmeier \& Smith (1978):

$$
100 K=2.1 M^{1.14}\left(10^{-4}\right)(12-a)+3.25(b-2)+2.5(c-3)
$$

Donde: $M=$ parámetro asociado al tamaño de las partículas. $M=(\%$ de limo modificado ${ }^{*}(\%$ limo+\%arena); \% limo modificado es el comprendido entre $0.1 \mathrm{y}$ $0.002 \mathrm{~mm}$.

$a=\%$ de materia orgánica

$b=$ código de estructura del suelo (1-granular muy fina; 2-granular fina; 3-granular media o gruesa; 4-en bloques, laminar o masiva)

$c=$ clase de permeabilidad del perfil (1- rápida; 2-moderada a rápida; 3-moderada; 4-lenta a moderada; 5-lenta; 6-muy lenta).

Los datos que contiene la ecuación se calcularon a partir del procesamiento de información edafológica correspondiente al área de estudio. La misma se obtuvo a través de muestreos realizados a campo, a lo largo de tres transectas que abarcaron las principales unidades ambientales de la microcuenca. Estas unidades fueron delimitadas considerando la información de la distribución de los suelos presentes en la microcuenca contenida en las cartas de suelos publicadas por INTA (INTA, 1992) a escala semidetallada (1:50.000), en las hojas 3760-22-1 "Base Azopardo" y 3760-22-3 "Chillar", además de aquella resultante del procesamiento, clasificación y análisis, de una imagen de satélite de alta resolución espacial.

El análisis de la imagen de satélite, que se realizó con el software Idrisi Kilimanjaro, contribuyó a identificar vías de escurrimiento temporarias como también las áreas de cerro caracterizadas por la presencia de roca y por cobertura vegetal natural de pastizal, además de las áreas de uso agrícola. Con tal finalidad, se empleó una imagen proveniente del satélite SPOT 5, cuya resolución espacial es de $10 \mathrm{~m}$ y que resulta adecuada para escalas de trabajo de semidetalle. Además, posee resolución espectral en bandas del visible, del infrarrojo cercano y del infrarrojo medio, cuya información es de utilidad para el análisis de la cobertura vegetal y áreas naturales de escurrimiento (Jensen, 1996). Se seleccionó una imagen correspondiente al mes de septiembre de 2012, posterior a los eventos de lluvia registrados durante el mes de agosto, lo que reflejó las condiciones de alta humedad en la cuenca y permitió la delimitación de los ambientes de interés. Para identificar las principales unidades ambientales se procedió de la siguiente forma: 
a- Selección del área de interés y procesamiento de las bandas a utilizar.

En primer lugar, se realizó una definición inicial rectangular del área de interés, sobre la cual se realizó la corrección geométrica de las bandas del satélite Spot a utilizar, a partir de puntos de control.

De acuerdo con la finalidad propuesta para este caso de análisis, se seleccionaron las bandas correspondientes sobre las cuales se aplicaron distintos procesamientos. Según el comportamiento espectral de la vegetación caracterizado por baja reflectividad en el espectro visible, alta en el infrarrojo cercano y baja, con algunos máximos relativos, en el infrarrojo medio de onda corta (Chuvieco, 2002), se seleccionaron las bandas del rojo y del infrarrojo cercano del satélite Spot. Con estas se calculó el índice de vegetación de diferencia normalizada (NDVI, Normalized Difference Vegetation Index), que consiste en un cociente entre dichas bandas. Este índice permite identificar áreas de vegetación en crecimiento activo, con presencia de suelo descubierto o con suelo húmedo, por lo que se incluyó en el proceso de clasificación.

Por otra parte, se aplicó un filtro digital adaptativo sobre la banda del infrarrojo cercano, que permitió remover el "ruido" en la misma, es decir los valores de los píxeles sin relación con la escena, y al mismo, tiempo suavizar píxeles relacionados con la imagen pero que poseen efecto de "ruido" aditivo o multiplicativo (Jensen, 1996). Esto posibilitó reducir la heterogeneidad en las clases resultantes del proceso de agrupamiento.

Además, la inclusión de la banda del verde, mejoró el agrupamiento de las clases a discriminar en dicha escena.

b- Clasificación de la imagen y elaboración del mapa de ambientes.

Se implementó un tipo de clasificación híbrida supervisada/no supervisada (Schowengerdt, 1997) utilizando las bandas seleccionadas y procesadas según los procedimientos del punto a-. Se produjo, en primer lugar, una clasificación no supervisada de los datos, que permitió obtener un mapa con agrupamientos no identificados de pixeles con características espectrales similares. Luego, se evaluó el mapa con datos de campo, referidos a los usos presentes en cada lote e incluyendo información de áreas de cerro y pastizal natural, y así se asignaron las clases a dichos agrupamientos. 
Una vez obtenida la imagen clasificada, fue superpuesta sobre el mapa de distribución de los tipos de suelos del área de estudio, que fue digitalizado a partir de las cartas publicadas por INTA. Así se obtuvo el mapa final de los ambientes principales de la microcuenca, que incluyó las siguientes unidades: áreas de cerros y pajonal, áreas de vaguadas, área del cauce principal de la microcuenca, áreas agrícolas de suelos someros y áreas agrícolas de suelos profundos.

Posteriormente, se realizaron observaciones de suelos en 12 sitios representativos de las diferentes unidades. En cada uno se describieron los horizontes superficiales y subsuperficiales en relación a propiedades edáficas tales como espesor de los mismos, tipo, clase y grado de la estructura presente, mientras que se realizó la estimación de la permeabilidad según con los criterios propuestos por Wischmeier et al. (1971). Paralelamente se tomaron muestras de dichos horizontes las cuales fueron analizadas luego en laboratorio, para determinar la distribución de partículas elementales (textura) por el método del hidrómetro (Bouyoucos, 1962) y el contenido de carbono orgánico según el método de Walkley \& Black (Nelson \& Sommers, 1996). A partir de estos datos, se calcularon los valores del factor $\mathrm{K}$ de acuerdo con la Ecuación (2.6). Luego se ponderaron por el área a la que se encontraban asociados, y finalmente se calculó el valor del factor $\mathrm{K}$ para la microcuenca.

\subsubsection{Factor de longitud e inclinación de la pendiente (LS)}

La longitud y la inclinación de la pendiente afectan la erosión por el agua. Su efecto fue evaluado de manera separada en las investigaciones realizadas durante el desarrollo de la USLE. Sin embargo, en las aplicaciones prácticas de esta ecuación, resulta más conveniente considerarlos de manera conjunta (Wischmeier \& Smith, 1978).

Para el cálculo de este factor de la MUSLE se emplearon las ecuaciones citadas por Gaspari et al. (2009) y Besteiro \& Gaspari (2012), las cuales, según mencionan estos autores, corresponden a modificaciones de la ecuación establecida por Wischmeier \& Smith (1978).

$$
L S=\left(\frac{\lambda}{22,1}\right)^{0,3} *\left(\frac{0,43+0,3 * s+0,043 * s^{2}}{6,613}\right) \text { para pendientes }<9 \%
$$




$$
\begin{gathered}
L S=\left(\frac{\lambda}{22,1}\right)^{0,3} *\left(\frac{s}{9}\right) \text { para pendientes }>9 \% \\
\lambda=\frac{0,5 * A}{L T C}
\end{gathered}
$$

Donde: $L S=$ factor de longitud e inclinación de la pendiente

$\lambda=$ longitud de la pendiente

$s=$ pendiente, expresada en porcentaje

$A=$ área del sector delimitado

$L T C=$ longitud total de los cursos que drenan por el área delimitada

Los datos necesarios para estos cálculos se obtuvieron a partir de la información topográfica contenida en las cartas del Instituto Geográfico Militar, hojas 3760-22-1 "Base Azopardo" y 3760-22-3 "Chillar", correspondientes al área de estudio. En primer lugar, se digitalizaron las curvas de nivel mediante el sistema de información geográfica (SIG) ILWIS 3.6. Luego, se realizó la interpolación de dichas curvas para obtener el modelo de elevación digital del terreno (DEM). A partir de éste se delimitó la microcuenca bajo estudio, para lo cual se aplicaron rutinas previas que involucraron la identificación de la dirección y la acumulación del flujo, la extracción de la red de drenaje y la identificación de los órdenes de los cursos de agua dentro de la red de drenaje. Asimismo, con la información del DEM se elaboró el mapa de pendientes a través del módulo "surface" del software de SIG Idrisi Kilimanjaro.

Con el objetivo de contemplar diferencias en cuanto a las pendientes y sus longitudes, y de acuerdo con las características físicas de la microcuenca, se la subdividió en 3 áreas: área superior, media e inferior. Entonces, para cada una se calculó la longitud total de las vaguadas que la recorrían, a partir de la red de drenaje antes mencionada. Este dato se relacionó con la superficie de cada área considerada según la Ecuación (2.9), y se obtuvieron los valores de longitud de la pendiente.

Luego, de cada área se extrajeron los sectores de pendientes menores que $9 \%$ y aquellos de pendientes superiores a $9 \%$, mediante distintos procedimientos en el software Idrisi. Se calcularon entonces las pendientes medias correspondientes a cada una de las zonas diferenciadas y se asociaron, junto con los valores de $\lambda$, a las Ecuaciones (2.7) y (2.8). Los valores de LS resultantes se ponderaron por las áreas que ocupaban dentro de los sectores de pendientes delimitados, se vincularon a la superficie total de la microcuenca, y 
así se obtuvo finalmente el valor ponderado de este factor para el cálculo de la producción de sedimentos a través de MUSLE.

\subsubsection{Factor de cultivo (C)}

Este factor evalúa de manera conjunta el efecto de la cobertura y su manejo, lo que comprende tipos de cultivos, rotaciones, manejo de los residuos, sistemas de labranza implementados, entre otros. Las diferentes combinaciones de la cobertura y su manejo tendrán una efectividad variable en cuanto al control de la erosión (Wischmeier \& Smith, 1978).

De acuerdo a lo que mencionan Smith et al., (1984) en la aplicación del modelo MUSLE, el cálculo de este factor se realizó a través de la información contenida en las tablas del manual 537 de la USLE. Ello requirió conocer los cultivos, las rotaciones, fechas de siembra, cosecha, rendimientos y sistemas de labranzas implementados en los lotes pertenecientes a la microcuenca durante el período de estudio. Esta información, además de aquella relacionada con la distribución y denominación de los lotes, fue relevada a través de entrevistas periódicas con los productores de los establecimientos del área de trabajo.

Para cada evento analizado, y a partir de los datos relevados, se elaboró una planilla en la que se establecieron las etapas de crecimiento en que se encontraban los cultivos en cada lote, incluyendo a los períodos de barbecho y de postcosecha. Dichas etapas se definieron a través de la cobertura porcentual de la superficie por parte del cultivo (Wischmeier \& Smith, 1978). El grado de cobertura correspondiente a cada cultivo en cada lote se determinó con información obtenida en relevamientos en el campo.

Luego, de acuerdo con la información contenida en las tablas se asignaron los coeficientes de relación de pérdida de suelo correspondientes. Estos son valores comparativos de la pérdida de suelo que ocurre bajo condiciones específicas de uso, manejo y rotaciones de cultivos en relación a una parcela mantenida bajo barbecho descubierto. Cabe aclarar que el factor $\mathrm{C}$ fue estimado a través de este valor individual, es decir, que no se realizó su cálculo como el promedio anual que considera la efectividad protectora de la cubierta vegetal para combinaciones de cultivos, manejos y patrones de lluvia. Esto se debe a que a través de MUSLE se realizan predicciones de la cantidad total de sedimentos a escala 
de eventos individuales, por lo cual, se tuvo en cuenta únicamente el estado en que estaban los cultivos al momento de la crecida, a través del coeficiente mencionado.

Por otra parte, se elaboró el mapa con la distribución de los lotes en la microcuenca. Este se realizó a partir de la digitalización sobre la imagen Spot que fue utilizada para la delimitación de los ambientes relacionada con la estimación del factor K. Así, se calcularon las superficies correspondientes a cada uno. Luego, junto con los coeficientes definidos para cada cultivo y las áreas asociadas, se calculó el valor de $\mathrm{C}$ ponderado para cada uno de los 17 eventos analizados.

\subsubsection{Factor de prácticas de conservación de suelos (P)}

Este factor considera el efecto protector que ejercen las prácticas específicas de conservación de suelos, como la realización de labranzas en contorno, cultivos en fajas o terrazas (Wischmeier \& Smith, 1978). La estimación del factor P, entonces, requiere conocer las prácticas específicas que son implementadas en el área de estudio. Para ello, junto con el relevamiento de información de los cultivos se tomaron datos de la implementación de dichas prácticas en la microcuenca.

Los valores de $\mathrm{P}$ se obtuvieron a partir de las tablas publicadas en el Manual de Agricultura 537 (Wischmier \& Smith, 1978). A cada lote delimitado dentro de la microcuenca se asignó el factor correspondiente, mientras que se obtuvo el $\mathrm{P}$ ponderado para el área de estudio al considerar la superficie de cada lote por el valor de $\mathrm{P}$ correspondiente.

\subsection{Análisis de los datos. Métodos estadísticos empleados}

Se aplicaron distintos procedimientos para analizar los resultados obtenidos mediante los monitoreos realizados. Para identificar los factores que explican la respuesta hidrológica y la respuesta en cuanto a la producción de sedimentos, la totalidad de los datos se analizó a través de una matriz de correlación de Pearson.

Asimismo, con el objetivo de evaluar las diferencias en la respuesta lluvia-escurrimientoconcentración y producción de sedimentos asociada a variaciones estacionales en la cobertura y en los patrones de precipitación, se establecieron 2 períodos de análisis. Dichos 
períodos se definieron como período de "media a baja cobertura" y período de "alta cobertura". El primero corresponde a los meses de mayo, junio, julio, agosto y septiembre, durante los cuales los suelos están en barbecho, se realizan las siembras de los cultivos de invierno y estos inician su crecimiento. Al mismo tiempo, en estos meses se registran las menores precipitaciones mensuales y también los menores $\mathrm{El}_{30}$ (Figuras 3.2 y 2.3, respectivamente). En cuanto al período de alta cobertura, incluyó a los meses de octubre, noviembre, diciembre, enero, febrero, marzo y abril, en los que los cultivos se encuentran en crecimiento activo y finalizan su ciclo. Además coincide con la época de mayores precipitaciones y $\mathrm{El}_{30}$ mensuales. Los datos se agruparon entonces de acuerdo con este criterio y se analizaron las relaciones mediante correlaciones de Pearson.

Los análisis de correlaciones antes detallados se complementaron con el estudio de relaciones a nivel de variables individuales a través de regresiones lineales simples y de regresiones no lineales.

Por otra parte, se realizó un agrupamiento de los eventos a través del método de estadística multivariada de análisis de cluster. Este método permite identificar la asociación natural de las observaciones, sin realizar presunciones previas de ellos e identificar patrones, en este caso, en cuanto a la respuesta lluvia-escurrimiento-producción de sedimentos. Se empleó el método de agrupamiento jerárquico de Ward, que ha resultado ser adecuado para el análisis de este tipo de datos (Giménez et al., 2012). Los datos se estandarizaron y se seleccionó la distancia Euclídea al cuadrado, como medida de la similitud entre los eventos a agrupar. 


\section{RESULTADOS}

\subsection{Precipitaciones y variables relacionadas}

\subsubsection{Caracterización de las precipitaciones en la zona}

\subsection{1.a. Análisis de dobles masas.}

La correlación obtenida entre la precipitación acumulada de las estaciones Cerro del Águila y Trapenses fue muy alta (Figura 3.1) lo que permitió el empleo de los datos de la estación Trapenses para los análisis realizados en la microcuenca.

\subsection{1.b. Distribución mensual de las precipitaciones}

La distribución de las precipitaciones medias mensuales registradas entre 1972 y 2012 (Figura 3.2) muestra que estas tienen sus mayores valores durante la primavera, el verano y el principio del otoño, entre los meses de octubre, noviembre, diciembre, enero, febrero, marzo y abril. El valor medio anual para dicho período fue de $905 \mathrm{~mm}$, mientras que el total anual para el año 2011 fue de $807 \mathrm{~mm}$ (11\% menor que el promedio anual) y para el año 2012 de $1351 \mathrm{~mm}$ (33\% superior al promedio anual). Asimismo, la precipitación del año 2012 superó en 40\% a la de 2011. Si se comparan entre sí las lluvias mensuales de 2011, 2012 y los promedios históricos, se observan diferencias destacables entre los valores de los meses de enero, mayo, agosto y diciembre. En el primer caso, la precipitación de enero de 2011 fue 53\% mayor que la correspondiente al mismo mes de 2012, y 57\% mayor que el promedio para el área. Para los 3 restantes, las lluvias de mayo, agosto y diciembre de 2012 superaron en 78\%, 96\% y 96\% respectivamente a las de iguales meses de 2011, en tanto que con respecto a las medias mensuales fueron $58 \%$, 82\% y 56\% superiores en dichos meses de 2012 . 


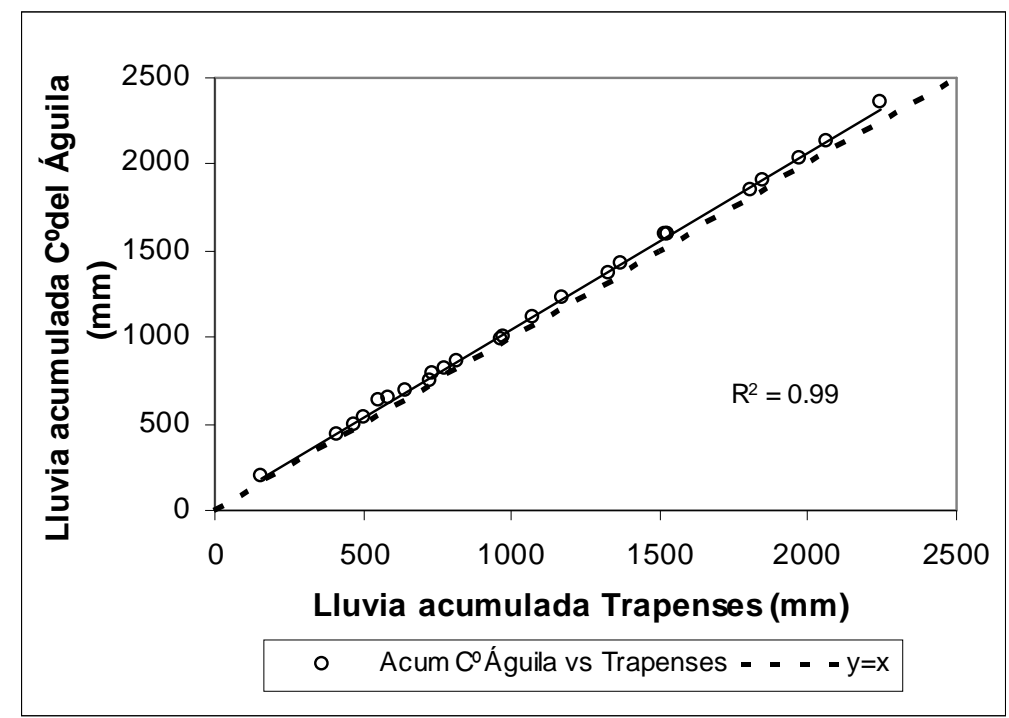

Figura 3.1. Diagrama del método de dobles masas: comparación de las precipitaciones mensuales acumuladas de las estaciones Cerro del Águila y Trapenses.

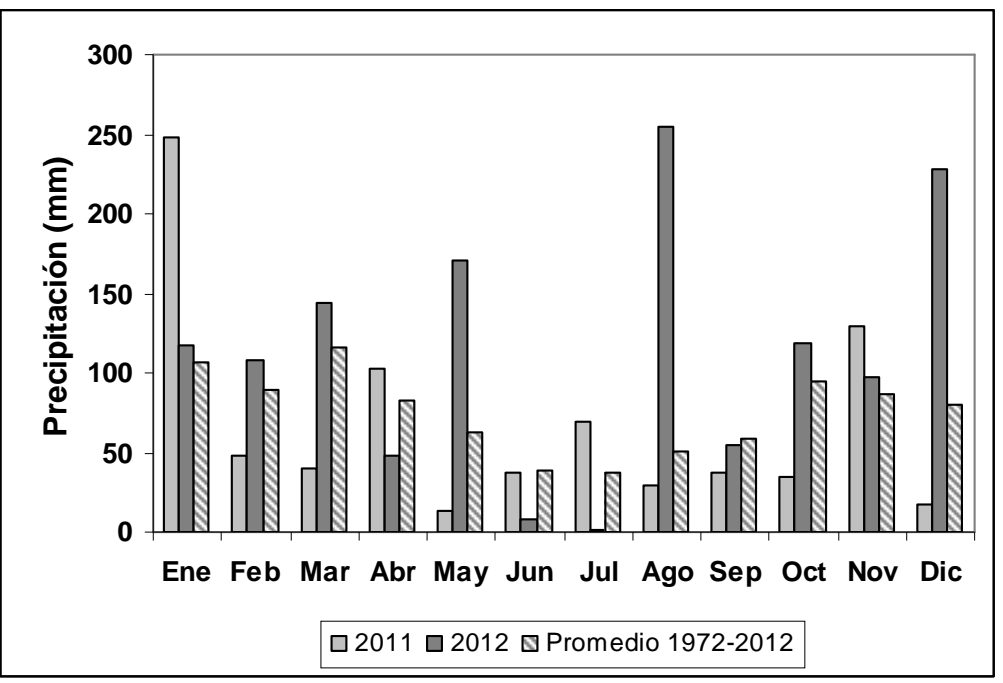

Figura 3.2. Precipitaciones mensuales de los años 2011 y 2012 y precipitaciones medias mensuales correspondientes al período 1972-2012 registradas para el área de estudio.

\subsection{1.c. Valores de $\mathrm{EI}_{30}$}

El valor anual calculado del índice $\mathrm{El}_{30}$ para el año 2011 fue de $3037 \mathrm{MJ} \mathrm{mm}$ (ha h) ${ }^{-1}$,

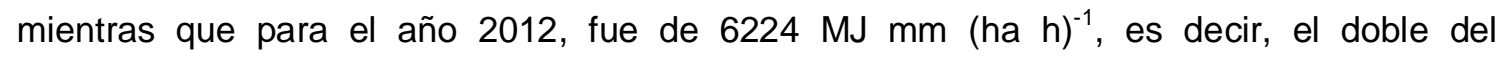
correspondiente al año 2011. Asimismo, estos resultados superan al valor calculado para un 
período de 13 años con datos observados para la localidad de Azul (Figura 2.1), que es de $2487 \mathrm{MJ} m \mathrm{~mm}(\text { ha h})^{-1}$ (Ares et al., 2010).

Por otra parte, en la Figura 3.3 se presenta de manera comparativa la distribución mensual de $\mathrm{El}_{30}$ para los años 2011, 2012 y la correspondiente al promedio calculado para el período de 13 años. Los registros del año 2011 muestran que el mes de enero fue el que presentó el máximo $\mathrm{El}_{30}$, que correspondió al 63\% del total para ese año. Por su parte, durante 2012, el $\mathrm{El}_{30}$ mostró diferencias más notables para los meses de mayo, agosto y diciembre, entre los cuales el de mayo superó en $31 \%$ al de ese mes de 2011 , y en $92 \%$ al promedio de 13 años para dicho mes. En cuanto a agosto de 2012, el índice fue $100 \%$ mayor que el correspondiente a 2011, dado que en ese mes no se registraron lluvias erosivas según el criterio expresado en la sección 2.2.2, y 87\% mayor que el promedio de 13 años para agosto. Mientras tanto, el $\mathrm{El}_{30}$ registrado en diciembre de 2012 representó el $35 \%$ del total para ese año, y superó en $100 \%$ y en $90 \%$ a los valores para ese mes de 2011 y a los de los 13 años analizados, respectivamente.

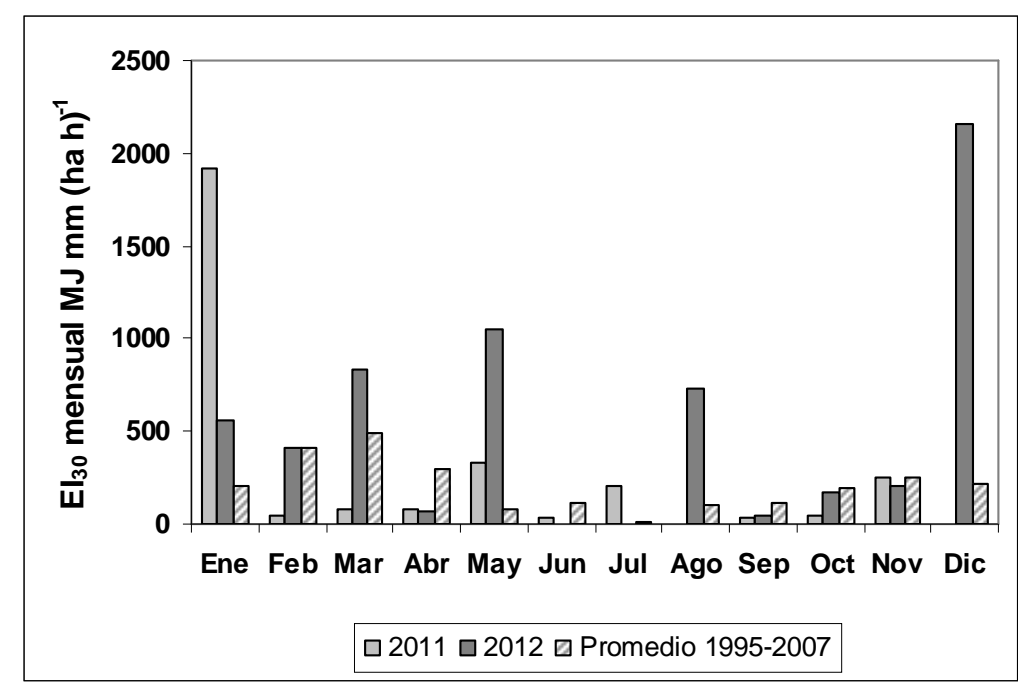

Figura 3.3. Valores mensuales del índice $\mathrm{El}_{30}$ correspondientes a los años 2011 y 2012 , comparados con los valores calculados por Ares et al. (2010) para la localidad de Azul en el período 1995-2007.

\subsection{Reserva de agua en el suelo}

La reserva de agua en el suelo media diaria fue de 40,4 mm para el año 2011, mientras que para el año 2012 fue de 63,1 mm, es decir $36 \%$ mayor que la calculada para 2011. Por otra 
parte, las medias diarias mensuales (Figura 3.4) muestran que esta variable superó los 40 $\mathrm{mm}$ en el mes de enero, y entre mayo y septiembre de 2011. Durante el año 2012 la reserva media diaria mensual se mantuvo por encima de $40 \mathrm{~mm}$ entre marzo y diciembre. El comportamiento mensual de esta variable se asocia a diferencias en la precipitación, la evapotranspiración y la relación que se da entre ellas.

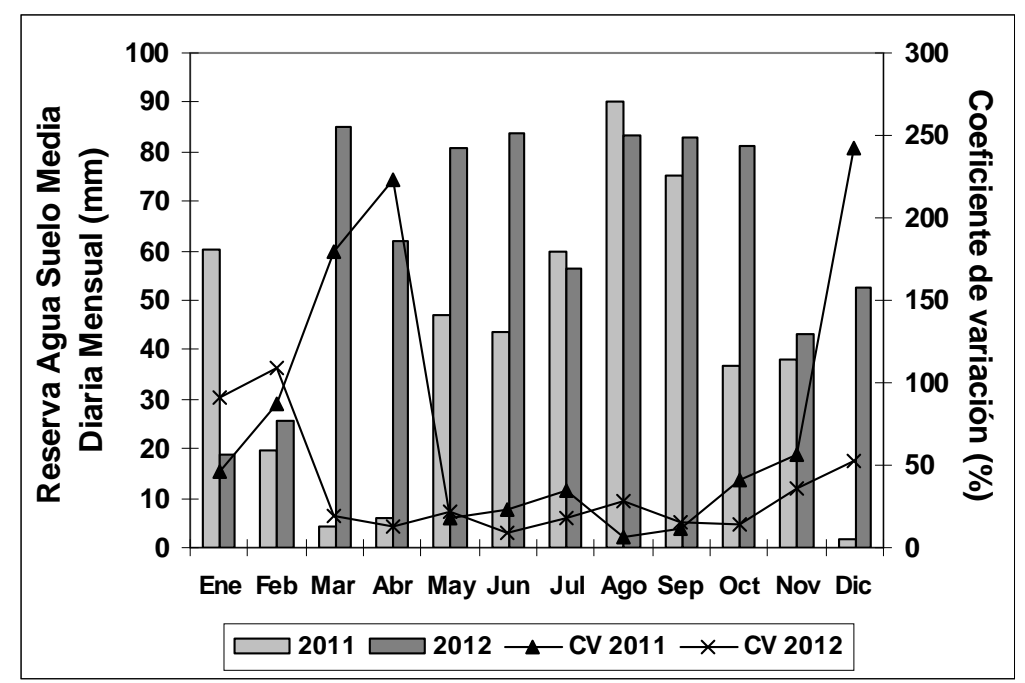

Figura 3.4. Valores medios diarios mensuales de reserva de agua en el suelo correspondientes a la microcuenca bajo estudio (y sus respectivos coeficientes de variación) calculados para los años 2011 y 2012.

\subsection{Eventos lluvia-escurrimiento-producción de sedimentos registrados}

Para este análisis se consideraron las muestras de agua de escurrimiento correspondientes a un total de 17 crecidas registradas en forma continua por el instrumental antes descripto durante el periodo 2011-2012. De dichos eventos 4 pertenecieron al año 2011 y 13 al año 2012, mientras que 6 se produjeron durante otoño-inverno y 11 durante primavera-verano. En la Tabla 3.1 se presentan los valores de las variables correspondientes a cada crecida estudiada y sus características principales se comentan a continuación.

El evento de mayor precipitación fue el registrado el 17/05/2012, con un total de 136,4 mm, y el de menor precipitación se produjo el 18/04/2012 con 17,8 mm. El 52,9\% de los eventos tuvo una magnitud menor que el promedio de precipitación de los 17 eventos considerados. En cuanto al $\mathrm{El}_{30}$, sus valores se encontraron entre 41,6 y $1144,5 \mathrm{MJ} \mathrm{mm}$ (ha h) ${ }^{-1}$, y 6 eventos $(35,3 \%)$ superaron el valor promedio calculado para esta variable. En tanto, la energía de las precipitaciones fue máxima durante la lluvia del día 17/05/2012, de 28 MJha-1, 
y fue mínima en la precipitación del 18/04/2012, de 3,9 $\mathrm{MJ} \mathrm{ha}^{-1}$, mientras que siete eventos presentaron valores por encima del promedio calculado para dicha variable, que fue de 10,5 $\mathrm{MJ} \mathrm{ha}^{-1}$. Por otra parte, la intensidad máxima en 30 minutos tuvo valores entre 57,2 y 7,2 $\mathrm{mm} \mathrm{h}^{-1}$, en tanto que la intensidad máxima en 10 minutos igualó o superó a la $\mathrm{I}_{30}$ y registró un máximo de 75,6 $\mathrm{mm} \mathrm{h}^{-1}$. La mayoría de los eventos presentaron intensidades máximas en 10 y 30 minutos por debajo de los promedios calculados para ambas variables, mientras que aquellos que superaron a los promedios correspondieron al semestre cálido.

La precipitación en los 5 días previos al evento presentó la más alta variabilidad, con un coeficiente de variación de $171 \%$ y 5 casos tuvieron valores mayores que el promedio calculado para la misma. En cambio, la reserva de agua en el suelo del día previo a la precipitación registró uno de los menores coeficientes de variación.

El escurrimiento fue máximo durante la crecida del día 17/05/2012 con 42,6 mm escurridos, mientras que 2 eventos coincidieron con la mínima lámina escurrida de 0,4 mm (19/07/2011 y 18/04/2012).

El caudal pico varió entre 4,6 y $0,1 \mathrm{~m}^{3} \mathrm{~s}^{-1}$ y 6 crecidas $(15 / 01 / 2011,17 / 05 / 2012,23 / 08 / 2012$, 05/12/2012, 19/12/2012 y 28/12/2012) superaron el caudal pico promedio que fue $1,3 \mathrm{~m}^{3} \mathrm{~s}^{-1}$. El caudal superficial medio osciló entre 1,2 y $0,02 \mathrm{~m}^{3} \mathrm{~s}^{-1}$, con un promedio de $0,3 \mathrm{~m}^{3} \mathrm{~s}^{-1}$, y las mismas 6 crecidas antes mencionadas presentaron un valor superior a este promedio. Asimismo, estos 6 eventos registraron coeficientes de escurrimiento mayores que el promedio, que fue de $15,4 \%$, en tanto que el coeficiente de escurrimiento estuvo comprendido entre 56,9 y $0,9 \%$. En cuanto al factor de escurrimiento, fue máximo durante el evento del 17/05/2012, y mínimo en el correspondiente al 18/04/2012. Además, tanto el escurrimiento como las variables asociadas antes mencionadas tuvieron coeficientes de variación superiores a $100 \%$.

Los tiempos correspondientes al pico de las crecidas se encontraron entre 17 y 2,5 horas. Por otra parte, el CN resultó entre 50 y 93, y el 53\% de los eventos registraron CN superiores a 70 , el promedio calculado para esta variable.

El factor de escurrimiento, fue máximo durante el evento del 17/05/2012, y mínimo en el correspondiente al 18/04/2012. Su promedio fue de 6012, y 5 eventos presentaron valores superiores a dicho promedio, los que correspondieron al 15/01/2011, 17/05/2012, 23/08/2012, 05/12/2012 y al 19/12/2012. 
Tabla 3.1. Características generales de los eventos registrados en la microcuenca del arroyo Videla.

\begin{tabular}{|c|c|c|c|c|c|c|c|c|c|c|c|c|c|c|c|c|c|c|}
\hline № evento & Fecha & $P$ & Dur & $\mathrm{El}_{30}$ & $E$ & $\mathrm{I}_{30}$ & $I_{10}$ & Pa5d & ResA & Esc & Qp & Qsm & CE & $\mathrm{Tp}$ & FactorEsc & $\mathrm{CN}$ & \begin{tabular}{|c|} 
CSS \\
discreto
\end{tabular} & $\begin{array}{c}\text { CSS } \\
\text { compuesto }\end{array}$ \\
\hline 1 & $15 / 01 / 2011$ & 106,6 & 14,0 & 1144,5 & 22,7 & 50,4 & 70,8 & 6,2 & 37,0 & 26,0 & 3,23 & 0,89 & 24,4 & 13 & 17256 & 63 & 1,94 & 0,85 \\
\hline 2 & $01 / 05 / 2011$ & 65,4 & 21,5 & 328,1 & 13,0 & 25,2 & 34,8 & 9 & 5,1 & 1,7 & 0,33 & 0,05 & 2,6 & 15 & 1055 & 53 & 1,86 & 0,48 \\
\hline 3 & $19 / 07 / 2011$ & 39,8 & 14,0 & 49,0 & 6,8 & 7,2 & 7,2 & 0,4 & 34,1 & 0,4 & 0,19 & 0,02 & 1,0 & 3 & 343 & 62 & 0,52 & 0,38 \\
\hline 4 & $08 / 11 / 2011$ & 63,6 & 24,0 & 84,1 & 11,1 & 7,6 & 12,0 & 35,2 & 22,5 & 0,6 & 0,17 & 0,03 & 0,9 & 17 & 394 & 50 & 0,76 & 0,84 \\
\hline 5 & $10 / 01 / 2012$ & 60,6 & 16,0 & 536,8 & 12,8 & 42,0 & 68,4 & 0 & 0,0 & 1,1 & 0,20 & 0,03 & 1,9 & 8 & 630 & 53 & 1 & 0,52 \\
\hline 6 & 05/03/2012 & 65,2 & 9,0 & 371,7 & 13,5 & 27,6 & 34,8 & 0 & 37,6 & 1,4 & 0,21 & 0,05 & 2,2 & 10 & 743 & 52 & 1,02 & 0,42 \\
\hline 7 & $11 / 03 / 2012$ & 33 & 10,0 & 204,7 & 6,4 & 32,0 & 73,2 & 17,6 & 96,3 & 1,2 & 0,20 & 0,03 & 3,7 & 8 & 591 & 71 & 0,56 & 0,52 \\
\hline 8 & $18 / 04 / 2012$ & 17,8 & 6,0 & 62,7 & 3,9 & 16,0 & 34,8 & 6,4 & 49,3 & 0,4 & 0,20 & 0,02 & 2,2 & 8 & 290 & 80 & 0,74 & 0,52 \\
\hline 9 & $17 / 05 / 2012$ & 136,4 & 42,0 & 1030,3 & 28,3 & 36,4 & 64,8 & 0,2 & 77,1 & 42,6 & 3,84 & 1,05 & 31,2 & 8 & 25061 & 62 & 8,54 & 1,12 \\
\hline 10 & $23 / 08 / 2012$ & 75,8 & 35,5 & 250,0 & 14,9 & 16,8 & 36,0 & 77,8 & 100,0 & 19,7 & 2,25 & 0,52 & 26,0 & 10,5 & 12077 & 72 & 0,62 & 0,57 \\
\hline 11 & 03/09/2012 & 29,4 & 24,5 & 41,6 & 5,0 & 8,4 & 12,0 & 0,4 & 85,7 & 4,2 & 0,80 & 0,12 & 14,5 & 11 & 2860 & 82 & 0,5 & 0,46 \\
\hline 12 & 05/10/2012 & 30 & 8,0 & 51,4 & 5,4 & 9,6 & 18,0 & 16,4 & 59,3 & 2,8 & 0,58 & 0,09 & 9,2 & 10 & 1879 & 78 & 0,64 & 0,56 \\
\hline 13 & $15 / 10 / 2012$ & 53,8 & 24,0 & 114,2 & 9,2 & 12,4 & 15,6 & 0 & 61,2 & 6,6 & 0,86 & 0,13 & 12,2 & 11 & 3808 & 69 & 0,58 & 0,54 \\
\hline 14 & $22 / 11 / 2012$ & 28,4 & 9,0 & 55,4 & 5,4 & 10,3 & 19,2 & 6,4 & 18,3 & 2,8 & 0,61 & 0,11 & 9,8 & 10 & 1947 & 79 & 0,6 & 0,52 \\
\hline 15 & $05 / 12 / 2012$ & 26,8 & 7,0 & 98,9 & 5,5 & 18,0 & 24,0 & 40,2 & 41,1 & 11,1 & 2,52 & 0,72 & 41,3 & 6 & 9316 & 92 & 3,74 & 1,62 \\
\hline 16 & 19/12/2012 & 38,6 & 5,0 & 557,1 & 9,7 & 57,2 & 68,4 & 23,2 & 21,2 & 22,0 & 4,60 & 1,17 & 56,9 & 2,5 & 19169 & 93 & 6,76 & 4,14 \\
\hline 17 & $28 / 12 / 2012$ & 23,6 & 3,0 & 213,0 & 5,8 & 36,8 & 75,6 & 77 & 90,0 & 5,1 & 1,67 & 0,50 & 21,4 & 3,5 & 4777 & 87 & 5,28 & 1,7 \\
\hline Promedio & & 52,6 & 16,0 & 305,5 & 10,5 & 24,3 & 39,4 & 18,6 & 49,2 & 8,8 & 1,3 & 0,3 & 15,4 & 9,1 & 6011,6 & 70,4 & 2,1 & 0,9 \\
\hline $\begin{array}{l}\text { Coef de } \\
\text { variación } \\
(\%)\end{array}$ & & 60 & 69 & 112 & 66 & 65 & 64 & 171 & 65 & 136 & 110 & 123 & 105 & 44 & 129 & 20 & 118 & 99 \\
\hline Mediana & & 39,8 & 14,0 & 204,7 & 9,2 & 18,0 & 34,8 & 6,4 & 41,1 & 2,8 & 0,61 & 0,11 & 9,8 & 10,0 & 1947,2 & 70,8 & 0,76 & 0,54 \\
\hline $\begin{array}{l}\text { Relación } \\
\text { promedio/ } \\
\text { mediana }\end{array}$ & & 1,3 & 1,1 & 1,5 & 1,1 & 1,4 & 1,1 & 2,9 & 1,2 & 3,2 & 2,1 & 3,1 & 1,6 & 0,9 & 3,1 & 1,0 & 2,8 & 1,7 \\
\hline
\end{tabular}


La concentración de material suspendido del muestreo discreto de las crecidas resultó entre 8,5 y $0,5 \mathrm{~g} \mathrm{l}^{-1}$ con un coeficiente de variación de $118,2 \%$, que indica la alta variabilidad registrada. De los 17 casos en estudio, en 4 de ellos (23,5\%) las concentraciones fueron mayores que el promedio, y corresponden a eventos producidos durante el año 2012. En cuanto al muestreo compuesto, las concentraciones estuvieron entre 4,1 y $0,4 \mathrm{~g} \mathrm{l}^{-1}$, y en 4 tormentas se superó la concentración promedio.

\subsubsection{Relaciones entre las variables estudiadas}

\subsection{1.a. Análisis a escala de eventos individuales}

Para identificar los factores que explican la respuesta hidrológica y la respuesta en cuanto a la producción de sedimentos se realizó una matriz de correlación de Pearson (Tabla 3.2). Los resultados obtenidos se detallan a continuación.

En cuanto a la respuesta hidrológica en la cuenca, el escurrimiento presentó las relaciones significativas más altas $(p<0,01)$ con la energía de la precipitación, con el índice de erosividad de las precipitaciones y con la precipitación, mientras que las relaciones con $I_{30}$ y la duración de la precipitación fueron significativas al nivel $p<0,05$ con coeficientes de correlación menores. El caudal pico y el caudal superficial medio mostraron correlaciones significativas $(p<0,01)$ con las variables $\mathrm{El}_{30} \mathrm{el}_{30}$, y solamente el caudal superficial medio demostró relación con $\mathrm{I}_{10}$, pero con coeficiente de correlación menor y significancia a nivel $p<0,05$. Con respecto al coeficiente de escurrimiento, únicamente se relacionó de manera significativa $(p<0,05)$ con la variable $I_{30}$ con un coeficiente de 0,5 , en tanto que las correlaciones fueron altas $y$ significativas $(p<0,01)$ en orden de importancia con el caudal pico, caudal medio y el escurrimiento. El tiempo al pico mostró correlación negativa y significativa a nivel $p<0,05$ con el $C N$, mientras que el factor de escurrimiento registró relaciones significativas $(p<0,01)$ con el escurrimiento, caudal pico, caudal superficial medio, coeficiente de escurrimiento, $\mathrm{El}_{30}$, E y precipitación total. EI CN presentó la correlación significativa más alta con el CE $(p<0,01)$ y con la precipitación y su energía, la correlación fue negativa y con significancia menor. 
Tabla 3.2. Coeficientes de Correlación de Pearson entre las variables analizadas. La correlación es significativa al nivel 0,01 para los números en negrita y al nivel 0,05 para los números en negrita cursiva.

\begin{tabular}{|c|c|c|c|c|c|c|c|c|c|c|c|c|c|c|c|c|c|}
\hline Variable & $P$ & Dur & $\mathrm{El}_{30}$ & E & $\mathrm{I}_{30}$ & $\mathrm{I}_{10}$ & $\mathrm{~Pa}$ 5d & ResA & Esc & $Q p$ & Qsm & CE & $\mathrm{Tp}$ & $\begin{array}{c}\text { Factor } \\
\text { Esc }\end{array}$ & $\mathrm{CN}$ & $\begin{array}{c}\text { CSS } \\
\text { discreto }\end{array}$ & $\begin{array}{c}\text { CSS } \\
\text { compuesto }\end{array}$ \\
\hline$P$ & 1,00 & & & & & & & & & & & & & & & & \\
\hline Dur & 0,71 & 1,00 & & & & & & & & & & & & & & & \\
\hline $\mathrm{El}_{30}$ & 0,83 & 0,30 & 1,00 & & & & & & & & & & & & & & \\
\hline$E$ & 0,99 & 0,64 & 0,89 & 1,00 & & & & & & & & & & & & & \\
\hline$I_{30}$ & 0,37 & $-0,16$ & 0,78 & 0,49 & 1,00 & & & & & & & & & & & & \\
\hline$l_{10}$ & 0,31 & $-0,12$ & 0,69 & 0,42 & 0,91 & 1,00 & & & & & & & & & & & \\
\hline Pa5d & $-0,16$ & $-0,03$ & $-0,19$ & $-0,14$ & 0,03 & 0,18 & 1,00 & & & & & & & & & & \\
\hline ResA & $-0,01$ & 0,27 & $-0,10$ & $-0,02$ & $-0,13$ & 0,13 & 0,43 & 1,00 & & & & & & & & & \\
\hline Esc & 0,74 & 0,50 & 0,78 & 0,79 & 0,51 & 0,44 & 0,08 & 0,24 & 1,00 & & & & & & & & \\
\hline Qp & 0,44 & 0,18 & 0,65 & 0,52 & 0,62 & 0,48 & 0,26 & 0,15 & 0,89 & 1,00 & & & & & & & \\
\hline Qsm & 0,45 & 0,14 & 0,67 & 0,54 & 0,65 & 0,52 & 0,26 & 0,13 & 0,89 & 0,99 & 1,00 & & & & & & \\
\hline CE & 0,15 & 0,01 & 0,39 & 0,23 & 0,50 & 0,35 & 0,35 & 0,15 & 0,71 & 0,94 & 0,92 & 1,00 & & & & & \\
\hline $\mathrm{Tp}$ & 0,35 & 0,43 & 0,03 & 0,27 & $-0,31$ & $-0,32$ & $-0,16$ & $-0,17$ & $-0,12$ & $-0,33$ & $-0,34$ & $-0,43$ & 1,00 & & & & \\
\hline FactorEsc & 0,64 & 0,37 & 0,76 & 0,70 & 0,58 & 0,48 & 0,13 & 0,18 & 0,98 & 0,96 & 0,96 & 0,82 & $-0,21$ & 1,00 & & & \\
\hline $\mathrm{CN}$ & $-0,58$ & $-0,43$ & $-0,28$ & $-0,51$ & 0,04 & 0,06 & 0,37 & 0,36 & 0,11 & 0,41 & 0,39 & 0,63 & $-0,58$ & 0,23 & 1,00 & & \\
\hline $\begin{array}{c}\text { CSS } \\
\text { discreto }\end{array}$ & 0,37 & 0,11 & 0,56 & 0,45 & 0,61 & 0,55 & 0,18 & 0,09 & 0,73 & 0,80 & 0,83 & 0,74 & $-0,45$ & 0,78 & 0,30 & 1,00 & \\
\hline $\begin{array}{c}\text { CSS } \\
\text { compuesto }\end{array}$ & $-0,09$ & $-0,29$ & 0,25 & 0,01 & 0,60 & 0,42 & 0,29 & $-0,11$ & 0,41 & 0,74 & 0,73 & 0,83 & $-0,52$ & 0,57 & 0,55 & 0,72 & 1,00 \\
\hline
\end{tabular}


Por otra parte, la humedad antecedente expresada a través de la precipitación de los 5 días previos, no se relacionó significativamente con el escurrimiento, el caudal pico, el caudal medio, el coeficiente de escorrentía ni con la concentración de sedimentos suspendidos, tanto del muestreo discreto como del compuesto. El mismo resultado se obtuvo cuando las condiciones antecedentes se valoraron mediante la reserva de agua en el suelo del día previo a la precipitación.

Con relación a la concentración de sedimentos suspendidos del muestreo discreto, las correlaciones fueron altas y significativas $(p<0,01)$, en orden de relevancia, con el caudal medio, el caudal pico, el factor de escurrimiento, el coeficiente de escurrimiento y el escurrimiento. Las correlaciones con las variables asociadas a la precipitación fueron significativas al nivel de $\mathrm{p}<0.05$ para los casos de $\mathrm{I}_{30}, \mathrm{El}_{30}$, e $\mathrm{I}_{10}$ con coeficientes de 0,61, 0,56 y 0,55 respectivamente, en tanto que la precipitación, su energía y la duración de la precipitación no mostraron relación significativa con la concentración de sedimentos suspendidos de este tipo de muestreo.

La concentración de sedimentos suspendidos del muestreo compuesto presentó correlaciones significativas $(p<0,01)$ en orden de importancia con el coeficiente de escurrimiento, el caudal pico, el caudal superficial medio y la concentración de sedimentos suspendidos del muestreo discreto. El tiempo al pico, el factor de escurrimiento y el CN registraron relaciones significativas con esta CSS pero a nivel $p<0,05$. Mientras tanto, si se consideran las variables asociadas a la precipitación, la correlación fue significativa únicamente con $I_{30}(p<0,05)$.

Por último, cabe mencionar que las variables precipitación, duración de la precipitación y energía total de la lluvia estuvieron correlacionadas entre sí de manera significativa $(p<0,01)$, aunque sin presentar una asociación estrecha con las intensidades máximas en 10 ó 30 minutos.

Este análisis general indica que las CSS del muestreo discreto y del compuesto poseen factores condicionantes diferenciales que justifican un estudio por separado de ambas variables. La concentración de sólidos suspendidos del muestreo discreto presenta más relaciones con significancia estadística con las variables asociadas a la precipitación y con aquellas que evalúan el escurrimiento de manera más directa (Esc, Qp, Qsm). Por su parte, la CSS del muestreo compuesto posee mayormente relaciones estadísticamente significativas con las variables del escurrimiento y otras 
asociadas como el CN o el tiempo al pico de la crecida. De acuerdo con lo anterior, la CSS discreta será utilizada en el análisis tendiente a contemplar diferencias en la concentración de sedimentos asociadas a variaciones estacionales en la cobertura, las lluvias y el escurrimiento y asimismo para evaluar distintas respuestas debido a interacciones lluvia-escorrentía. Luego, considerando que el muestreo compuesto se realizó durante un período mayor durante la crecida, estos datos de CSS serán empleados para realizar cálculos de producción de sedimentos, a partir de datos de concentración y escurrimiento. Dichas estimaciones serán comparadas con las variables consideradas en este estudio, como también con los valores estimados de sedimentos producidos en las tormentas a través de la aplicación del modelo MUSLE.

\subsection{1.b. Análisis estacional: períodos de baja a media cobertura y de alta cobertura}

Como se detalló en la sección anterior, ingresaron en este análisis de correlación las variables de la precipitación, las vinculadas al escurrimiento y la concentración del muestreo discreto. Debido a que la lluvia de los 5 días previos y la reserva de agua en el suelo del día previo a la precipitación no registraron relaciones significativas con las variables antes mencionadas, no fueron incluidas en este caso.

En cuanto al período de baja a media cobertura, los datos correspondientes a 5 eventos ingresaron en este análisis (eventos № 2, 3,9, 10 y 11), y la matriz de correlación de Pearson se presenta en la Tabla 3.3. La misma muestra que la precipitación, su $\mathrm{E}$ y duración se relacionaron de manera significativa con el escurrimiento, el caudal pico y el caudal superficial medio. Para $\mathrm{El}_{30}$ como para $\mathrm{I}_{10}$ la correlación fue significativa con el escurrimiento y el Qsm $(p<0,05)$, mientras que únicamente la duración de la precipitación se relacionó significativamente con el coeficiente de escurrimiento $(p<0,01)$. El factor de escurrimiento presentó relaciones significativas a nivel $p<0,05$ con la lluvia total, su duración, $E_{30}$, $E$ y con $I_{10}$. Sin embargo, $I_{30}$ fue la única variable asociada a la precipitación que no mostró estar estadísticamente relacionada al escurrimiento, Qp, Qsm, CE y factor de escurrimiento.

Tanto el tiempo al pico como el $\mathrm{CN}$ no estuvieron relacionados de manera significativa con el resto de las variables consideradas.

La concentración del muestreo discreto se correlacionó significativamente con $\mathrm{El}_{30}$ $(p<0,01)$, E, Ptot y con $\mathrm{I}_{30}$ a nivel $\mathrm{p}<0,05$, y no presentó relaciones significativas con las variables del escurrimiento. 
Tabla 3.3. Coeficientes de Correlación de Pearson entre las variables analizadas considerando los eventos del período de baja a media cobertura.

La correlación es significativa al nivel 0,01 para los números en negrita y al nivel 0,05 para los números en negrita cursiva.

\begin{tabular}{|c|c|c|c|c|c|c|c|c|c|c|c|c|c|c|}
\hline Variable & $P$ & Dur & $\mathrm{El}_{30}$ & $E$ & $\mathrm{I}_{30}$ & $\mathrm{l}_{10}$ & Esc & Qp & Qsm & CE & Tp & $\begin{array}{c}\text { Factor } \\
\text { Esc }\end{array}$ & $\mathrm{CN}$ & $\begin{array}{c}\text { CSS } \\
\text { discreto }\end{array}$ \\
\hline$P$ & 1,00 & & & & & & & & & & & & & \\
\hline Dur & 0,84 & 1,00 & & & & & & & & & & & & \\
\hline $\mathrm{El}_{30}$ & 0,98 & 0,78 & 1,00 & & & & & & & & & & & \\
\hline$E$ & 1,00 & 0,84 & 0,98 & 1,00 & & & & & & & & & & \\
\hline $\mathrm{I}_{30}$ & 0,93 & 0,71 & 0,94 & 0,94 & 1,00 & & & & & & & & & \\
\hline $\mathrm{I}_{10}$ & 0,97 & 0,86 & 0,95 & 0,98 & 0,97 & 1,00 & & & & & & & & \\
\hline Esc & 0,93 & 0,93 & 0,90 & 0,93 & 0,77 & 0,89 & 1,00 & & & & & & & \\
\hline$Q p$ & 0,89 & 0,96 & 0,84 & 0,89 & 0,71 & 0,86 & 0,99 & 1,00 & & & & & & \\
\hline Qsm & 0,93 & 0,94 & 0,89 & 0,92 & 0,76 & 0,88 & 1,00 & 0,99 & 1,00 & & & & & \\
\hline CE & 0,72 & 0,97 & 0,66 & 0,72 & 0,53 & 0,72 & 0,90 & 0,95 & 0,91 & 1,00 & & & & \\
\hline Tp & 0,02 & 0,19 & 0,04 & 0,05 & 0,31 & 0,25 & $-0,10$ & $-0,07$ & $-0,09$ & 0,04 & 1,00 & & & \\
\hline Factor Esc & 0,93 & 0,94 & 0,90 & 0,92 & 0,76 & 0,88 & 1,00 & 0,99 & 1,00 & 0,91 & $-0,10$ & 1,00 & & \\
\hline $\mathrm{CN}$ & $-0,38$ & 0,15 & $-0,38$ & $-0,38$ & $-0,53$ & $-0,36$ & $-0,03$ & 0,08 & $-0,01$ & 0,35 & $-0,05$ & $-0,01$ & 1,00 & \\
\hline $\begin{array}{c}\text { CSS } \\
\text { discreto }\end{array}$ & 0,92 & 0,70 & 0,98 & 0,92 & 0,88 & 0,87 & 0,87 & 0,80 & 0,86 & 0,61 & $-0,08$ & 0,86 & $-0,33$ & 1,00 \\
\hline
\end{tabular}

Con respecto al período de alta cobertura, se analizaron en este caso las variables correspondientes a 12 eventos (eventos № 1, 4, 5, 6, 7, 8, 12, 13, 14, 15, 16 y 17) cuyas correlaciones resultantes se muestran en la Tabla 3.4. En contraste con lo hallado para el período de baja a media cobertura, la intensidad máxima de la lluvia en 30 minutos se correlacionó de manera significativa $(p<0,05)$ con el escurrimiento, el caudal pico, el caudal superficial medio y el factor de escurrimiento. En tanto, el índice $\mathrm{El}_{30}$ mostró relación significativa con el escurrimiento $(\mathrm{p}<0,01)$ y con el factor de escurrimiento $(p<0,05)$, en tanto que las restantes variables asociadas a la precipitación no se correlacionaron con aquellas vinculadas al escurrimiento.

Por otro lado, las variables caudal pico, caudal superficial medio y el coeficiente de escurrimiento, el tiempo al pico (de manera negativa) y el factor de escurrimiento presentaron relaciones significativas $(p<0,01)$ con la concentración del muestreo discreto, al igual que el escurrimiento, y el $\mathrm{CN}$ pero con un nivel de significancia menor 
$(p<0,05)$. De las variables de la precipitación, únicamente $I_{30}$ registró correlación significativa con dicha concentración.

En este caso, el tiempo al pico se correlacionó significativamente con la precipitación total y su duración, y con el coeficiente de escurrimiento, pero con este último de manera negativa. En cuanto al CN, presentó relaciones significativas con la lluvia, su duración, y la energía de forma negativa y a nivel $p<0,05$, mientras que las relaciones con el CE y Tp también fueron significativas, con signo positivo y negativo respectivamente.

Tabla 3.4. Coeficientes de Correlación de Pearson entre las variables analizadas considerando los eventos del período de alta cobertura.

La correlación es significativa al nivel 0,01 para los números en negrita y al nivel 0,05 para los números en negrita cursiva.

\begin{tabular}{|c|c|c|c|c|c|c|c|c|c|c|c|c|c|c|}
\hline Variable & $P$ & Dur & $\mathrm{El}_{30}$ & $E$ & $\mathrm{I}_{30}$ & $\mathrm{I}_{10}$ & Esc & Qp & Qsm & CE & Tp & $\begin{array}{c}\text { Factor } \\
\text { Esc }\end{array}$ & $\mathrm{CN}$ & $\begin{array}{c}\text { CSS } \\
\text { discreto }\end{array}$ \\
\hline$P$ & 1,00 & & & & & & & & & & & & & \\
\hline Dur & 0,56 & 1,00 & & & & & & & & & & & & \\
\hline $\mathrm{El}_{30}$ & 0,78 & 0,03 & 1,00 & & & & & & & & & & & \\
\hline$E$ & 0,98 & 0,40 & 0,89 & 1,00 & & & & & & & & & & \\
\hline$I_{30}$ & 0,37 & $-0,30$ & 0,82 & 0,53 & 1,00 & & & & & & & & & \\
\hline $\mathrm{I}_{10}$ & 0,17 & $-0,37$ & 0,64 & 0,32 & 0,90 & 1,00 & & & & & & & & \\
\hline Esc & 0,45 & $-0,12$ & 0,75 & 0,56 & 0,66 & 0,39 & 1,00 & & & & & & & \\
\hline Qp & 0,17 & $-0,31$ & 0,55 & 0,30 & 0,65 & 0,39 & 0,93 & 1,00 & & & & & & \\
\hline Qsm & 0,18 & $-0,35$ & 0,57 & 0,31 & 0,67 & 0,43 & 0,93 & 0,99 & 1,00 & & & & & \\
\hline CE & $-0,08$ & $-0,40$ & 0,31 & 0,04 & 0,51 & 0,27 & 0,79 & 0,95 & 0,94 & 1,00 & & & & \\
\hline $\mathrm{Tp}$ & 0,58 & 0,77 & 0,03 & 0,42 & $-0,48$ & $-0,52$ & $-0,19$ & $-0,45$ & $-0,46$ & $-0,59$ & 1.00 & & & \\
\hline $\begin{array}{l}\text { Factor } \\
\text { Esc }\end{array}$ & 0,33 & $-0,21$ & 0,68 & 0,45 & 0,68 & 0,40 & 0,98 & 0,98 & 0,97 & 0,88 & $-0,31$ & 1,00 & & \\
\hline $\mathrm{CN}$ & $-0,69$ & $-0,69$ & $-0,24$ & $-0,59$ & 0,09 & 0,10 & 0,32 & 0,54 & 0,54 & 0,70 & $-0,74$ & 0,42 & 1,00 & \\
\hline $\begin{array}{c}\text { CSS } \\
\text { discreto }\end{array}$ & $-0,19$ & $-0,52$ & 0,25 & $-0,04$ & 0,61 & 0,47 & 0,59 & 0,83 & 0,84 & 0,87 & $-0,73$ & 0,71 & 0,64 & 1,00 \\
\hline
\end{tabular}




\subsection{Agrupamiento de los eventos de acuerdo a la interacción lluvia- escorrentía-producción de sedimentos mediante análisis de cluster}

De acuerdo con lo planteado en el punto 3.3.1.a., este análisis se realizó considerando las variables asociadas a la precipitación y aquellas relacionadas al escurrimiento, entre las que se incluyó también al CE. Al igual que en el análisis estacional, se excluyó a las variables que valoran la humedad antecedente.

En la Figura 3.5 se muestra el resultado obtenido, en el que se diferencian 4 grupos si se considera la distancia de 5 . El análisis de los promedios de las variables correspondientes a los casos que resultaron incluidos dentro de cada grupo (Tabla 3.5) permite interpretar los diferentes agrupamientos, tendientes a caracterizar las respuestas en cuanto a la producción de sedimentos en la microcuenca en relación a los factores considerados.

En el grupo A se ubicaron los casos 12, 14, 3, 8, 11, 13 y 4. Estos son eventos que presentaron la menor concentración del muestreo discreto en promedio, con valores intermedios de escurrimiento, Qp y CE, pero con valores bajos de precipitación y de las variables relacionadas. En el grupo $B$, coincidieron los casos 2, 6, 5 y 7, los que mostraron concentración media de sedimentos suspendidos, los menores promedios de escurrimiento y variables asociadas, y promedios intermedios a altos de precipitación, $\mathrm{El}_{30}, \mathrm{E}, \mathrm{I}_{30} \mathrm{e}_{10}$. En cuanto a los grupos $\mathrm{C}$ y $\mathrm{D}$, reunieron a los eventos 1 y 9 , y 15, 17, 10 y 16 respectivamente, correspondientes a los de mayor concentración en promedio, con los mayores promedios de escurrimiento, Qp, Qm y CE. Sin embargo, los grupos se diferenciarían porque en $\mathrm{C}$ se ubicaron aquellos de máxima precipitación y variables asociadas, en tanto que $\mathrm{D}$ comprendió eventos de intermedia a baja magnitud de precipitación, $\mathrm{El}_{30}$ y $\mathrm{E}$. 


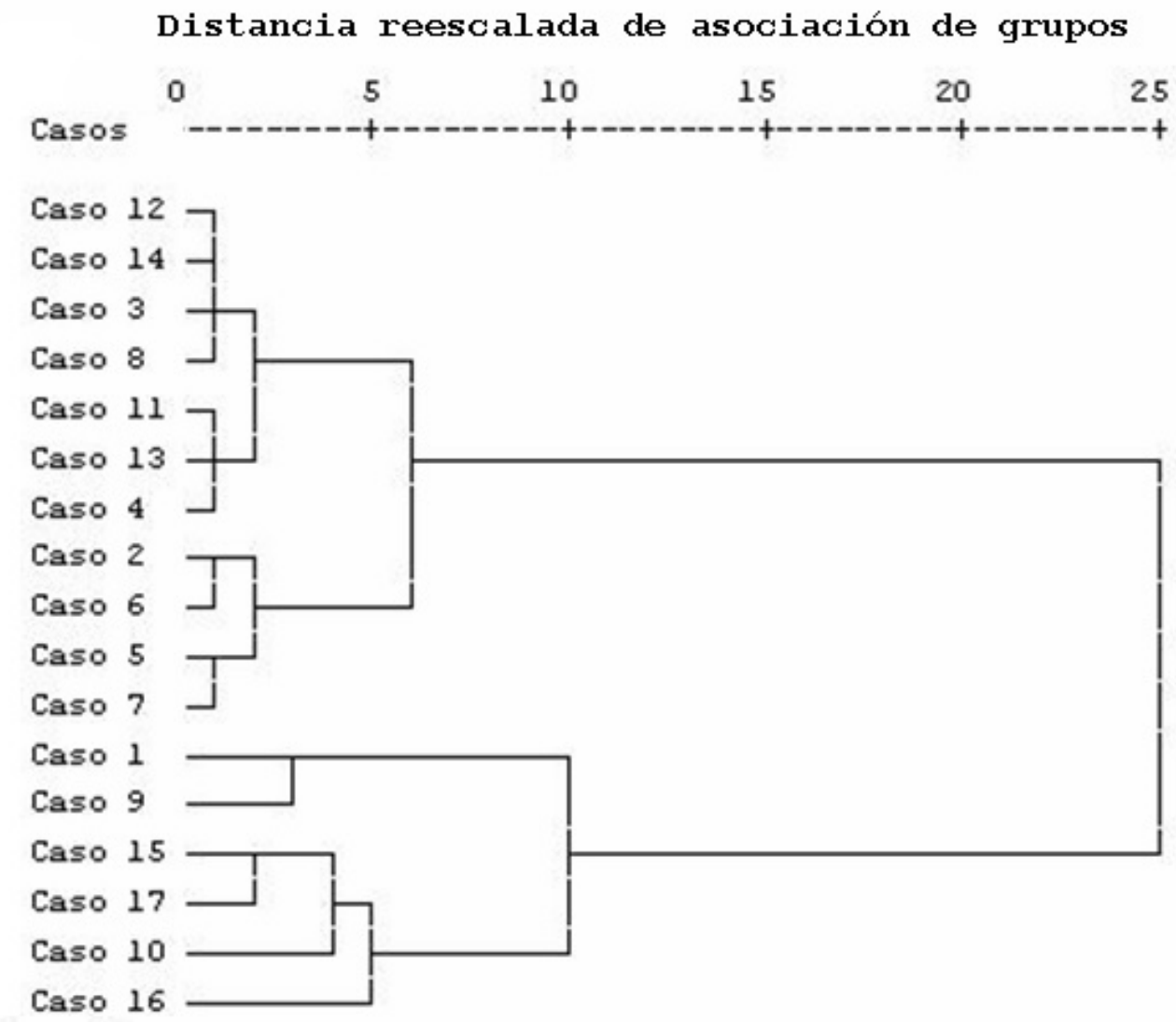

Figura 3.5. Dendrograma del análisis de cluster sobre los datos de precipitación, escurrimiento y variables asociadas a estos y concentración de sólidos suspendidos. 
Tabla 3.5. Promedios y coeficientes de variación (CV) de las variables correspondientes a los casos incluidos dentro de cada grupo obtenido a través del análisis de cluster.

\begin{tabular}{|c|c|c|c|c|c|c|c|c|c|c|c|c|c|c|c|c|c|c|c|c|c|c|c|}
\hline \multirow[b]{2}{*}{ Grupo } & \multirow[b]{2}{*}{$\begin{array}{l}\text { Casos/ } \\
\text { Eventos }\end{array}$} & \multicolumn{2}{|l|}{ Ptot } & \multicolumn{2}{|l|}{ Dur } & \multicolumn{2}{|l|}{$\mathrm{El}_{30}$} & \multicolumn{2}{|l|}{$E$} & \multicolumn{2}{|l|}{$I_{30}$} & \multicolumn{2}{|l|}{$I_{10}$} & \multicolumn{2}{|l|}{ Esc } & \multicolumn{2}{|l|}{$Q p$} & \multicolumn{2}{|c|}{ Qsm } & \multicolumn{2}{|l|}{$\mathrm{CE}$} & \multicolumn{2}{|l|}{ CSS } \\
\hline & & Promedio & $\begin{array}{l}\mathrm{CV} \\
(\%) \\
\end{array}$ & Promedio & $\begin{array}{l}\mathrm{CV} \\
(\%)\end{array}$ & Promedio & \begin{tabular}{|c|}
$\mathrm{CV}$ \\
$(\%)$ \\
\end{tabular} & Promedio & $\begin{array}{l}\mathrm{CV} \\
(\%) \\
\end{array}$ & Promedio & $\begin{array}{l}\mathrm{CV} \\
(\%)\end{array}$ & Promedio & $\begin{array}{l}\mathrm{CV} \\
(\%)\end{array}$ & Promedio & $\begin{array}{l}\mathrm{CV} \\
(\%) \\
\end{array}$ & Promedio & $\begin{array}{l}\mathrm{CV} \\
(\%)\end{array}$ & Promedio & $\begin{array}{l}\mathrm{CV} \\
(\%)\end{array}$ & Promedio & $\begin{array}{l}\mathrm{CV} \\
(\%) \\
\end{array}$ & Promedio & $\begin{array}{l}\mathrm{CV} \\
(\%)\end{array}$ \\
\hline A & $\begin{array}{c}12-14-3- \\
8-11-13- \\
4 \\
\end{array}$ & 37,5 & 42,7 & 15,6 & 53,3 & 65,5 & 38,8 & 6,7 & 38,5 & 10,2 & 30,4 & 17 & 52,2 & 2,5 & 91,4 & 0,5 & 64,3 & 0,08 & 62,7 & 7,1 & 79,2 & 0,6 & 16,2 \\
\hline B & $2-6-5-7$ & 56,1 & 27,7 & 14,1 & 41,1 & 360,3 & 38,1 & 11,4 & 29,4 & 31,7 & 23,4 & 52,8 & 39,5 & 1,4 & 18,7 & 0,2 & 32,1 & 0,04 & 31,2 & 2,6 & 31,5 & 1,1 & 48,9 \\
\hline $\mathrm{C}$ & $1-9$ & 121,5 & 17,3 & 28 & 70,7 & 1087,4 & 7,4 & 25,5 & 15,5 & 43,4 & 22,8 & 67,8 & 6,3 & 34,3 & 34,2 & 3,5 & 12,2 & 0,97 & 12,2 & 27,8 & 17,4 & 5,2 & 89,1 \\
\hline D & $\begin{array}{c}15-17- \\
10-16\end{array}$ & 41,2 & 58,1 & 12,6 & 122 & 279,7 & 70 & 9 & 48,9 & 32,2 & 59,1 & 51 & 48,8 & 14,4 & 54,2 & 2,8 & 46,3 & 0,7 & 42,5 & 36,4 & 44,2 & 4,1 & 64,1 \\
\hline
\end{tabular}




\subsection{Producción de sedimentos estimada}

La producción de sedimentos estimada varió entre 486,7 y 0,8 toneladas, con un promedio de 66,3. Cuatro de los eventos registrados superaron ese valor, los casos 1, 9, 15 y 16, mientras que el correspondiente al 19/12/2012 tuvo el mayor valor. Asimismo el coeficiente de variación resultante fue alto y cercano a $200 \%$.

Tabla 3.6. Producción total de sedimentos estimada, expresada en toneladas correspondientes a los 17 eventos registrados en la microcuenca bajo estudio.

\begin{tabular}{|c|c|c|}
\hline № evento & Fecha & $\begin{array}{l}\text { Prod } \\
\text { SedEst }\end{array}$ \\
\hline 1 & $15 / 01 / 2011$ & 119,4 \\
\hline 2 & $01 / 05 / 2011$ & 4,4 \\
\hline 3 & $19 / 07 / 2011$ & 0,8 \\
\hline 4 & 08/11/2011 & 2,6 \\
\hline 5 & $10 / 01 / 2012$ & 3,1 \\
\hline 6 & $05 / 03 / 2012$ & 3,2 \\
\hline 7 & $11 / 03 / 2012$ & 3,4 \\
\hline 8 & $18 / 04 / 2012$ & 1,1 \\
\hline 9 & $17 / 05 / 2012$ & 255,1 \\
\hline 10 & $23 / 08 / 2012$ & 60,1 \\
\hline 11 & $03 / 09 / 2012$ & 10,5 \\
\hline 12 & $05 / 10 / 2012$ & 8,2 \\
\hline 13 & $15 / 10 / 2012$ & 18,9 \\
\hline 14 & $22 / 11 / 2012$ & 7,7 \\
\hline 15 & $05 / 12 / 2012$ & 95,9 \\
\hline 16 & 19/12/2012 & 486,7 \\
\hline 17 & $28 / 12 / 2012$ & 46,0 \\
\hline Promedio & & 66,3 \\
\hline $\begin{array}{l}\text { Coef de } \\
\text { variación } \\
(\%)\end{array}$ & & 191,2 \\
\hline Mediana & & 8,2 \\
\hline $\begin{array}{l}\text { Relación } \\
\text { promedio/ } \\
\text { mediana }\end{array}$ & & 8,0 \\
\hline
\end{tabular}

\subsubsection{Relaciones entre la producción de sedimentos estimada y las variables estudiadas}

\subsection{1.a. Análisis a escala de eventos individuales}


Con el propósito de identificar las variables que se relacionaban más estrechamente con la producción de sedimentos estimada, se analizó una matriz de correlación de Pearson (Tabla 3.7). En cuanto a las variables asociadas a la precipitación, se observa que la relación fue significativa con $\mathrm{I}_{30}(\mathrm{p}<0,01)$ y con el índice $\mathrm{El}_{30}(\mathrm{p}<0,05)$. El escurrimiento, el caudal pico, el caudal superficial medio, el coeficiente de escorrentía y el factor de escurrimiento presentaron relaciones significativas a nivel $p<0,01$ con la estimación de producción de sedimentos realizada. En tanto, las variables que evalúan la humedad antecedente, al igual que el $\mathrm{CN}$ y el tiempo al pico no mostraron estar estadísticamente relacionadas con la producción estimada.

Tabla 3.7. Coeficientes de Correlación de Pearson entre las variables analizadas y la producción de sedimentos estimada.

La correlación es significativa al nivel 0,01 para los números en negrita y al nivel 0,05 para los números en negrita cursiva.

\begin{tabular}{|c|c|}
\hline Variable & $\begin{array}{c}\text { Prod } \\
\text { SedEst }\end{array}$ \\
\hline P & 0,28 \\
\hline Dur & 0,04 \\
\hline El $_{30}$ & $\mathbf{0 , 5 4}$ \\
\hline E & 0,37 \\
\hline I $_{30}$ & $\mathbf{0 , 6 6}$ \\
\hline $\mathrm{I}_{10}$ & 0,46 \\
\hline Pa5d & 0,07 \\
\hline ResA & $-0,06$ \\
\hline Esc & $\mathbf{0 , 7 2}$ \\
\hline Qp & $\mathbf{0 , 8 9}$ \\
\hline Qsm & $\mathbf{0 , 8 7}$ \\
\hline CE & $\mathbf{0 , 8 6}$ \\
\hline Tp & $-0,42$ \\
\hline Factor Esc & $\mathbf{0 , 8 2}$ \\
\hline CN & 0,38 \\
\hline ProdSed & 1,00 \\
\hline Est & \\
\hline
\end{tabular}


3.5.1.b. Análisis a escala estacional: períodos de baja a media cobertura y de alta cobertura

Para evaluar posibles diferencias en las relaciones entre la producción de sedimentos y las distintas variables debido a la estacionalidad en la cobertura y en los patrones de la precipitación se analizaron también correlaciones de Pearson (Tabla 3.8).

Tabla 3.8. Coeficientes de Correlación de Pearson entre las variables analizadas y la producción de sedimentos estimada para los períodos de alta cobertura y de media a baja cobertura.

La correlación es significativa al nivel 0,01 para los números en negrita y al nivel 0,05 para los números en negrita cursiva.

\begin{tabular}{|c|c|c|}
\hline \multirow[t]{2}{*}{ Variable } & $\begin{array}{c}\text { Período de alta } \\
\text { Cobertura }\end{array}$ & $\begin{array}{c}\text { Período de } \\
\text { media a baja } \\
\text { cobertura }\end{array}$ \\
\hline & ProdSedEst & ProdSedEst \\
\hline$P$ & 0,03 & 0,94 \\
\hline Dur & $-0,31$ & 0,85 \\
\hline $\mathrm{El}_{30}$ & 0,41 & 0,95 \\
\hline E & 0,15 & 0,94 \\
\hline$I_{30}$ & 0,65 & 0,81 \\
\hline $\mathrm{I}_{10}$ & 0,37 & 0,88 \\
\hline Pa5d & 0,13 & $-0,07$ \\
\hline ResA & $-0,24$ & 0,39 \\
\hline Esc & 0,75 & 0,98 \\
\hline Qp & 0,89 & 0,94 \\
\hline Qsm & 0,85 & 0,97 \\
\hline CE & 0,88 & 0,80 \\
\hline $\mathrm{Tp}$ & $-0,52$ & $-0,15$ \\
\hline Factor Esc & 0,85 & 0,97 \\
\hline $\mathrm{CN}$ & 0,49 & $-0,13$ \\
\hline $\begin{array}{l}\text { ProdSed } \\
\text { Est }\end{array}$ & 1,00 & 1,00 \\
\hline
\end{tabular}


En cuanto a las variables del escurrimiento, para los eventos ocurridos durante momentos de alta cobertura, el Esc, Qp, Qsm, el CE y el factor de escurrimiento mostraron relaciones significativas con la producción de sedimentos estimada, a nivel $p<0,01$. Para el período de baja a media cobertura las variables que se encontraron correlacionadas con la producción de sedimentos fueron el Esc, Qp, Qsm y el factor de escurrimiento.

Por último, no se registraron relaciones entre las estimaciones de producción de sedimentos y el $\mathrm{CN}$, el tiempo al pico y las variables que valoran la humedad antecedente.

\subsection{Parámetros del modelo MUSLE}

\subsubsection{Factor escurrimiento}

Los resultados correspondientes a este factor fueron ya fueron presentados en la sección 3.3 de este trabajo de Tesis.

\subsubsection{Factor $K$}

Los resultados obtenidos para las 12 observaciones de suelos realizadas en la microcuenca indican que, en términos generales, la textura de los horizontes superficiales es franca o franco-arcillosa, con espesores entre 25 y $30 \mathrm{~cm}$, y con contenidos de materia orgánica entre 6 y 7\%. Los suelos presentan estructuras superficiales en bloques angulares 0 subangulares medios, mientras que la permeabilidad fue clasificada como moderada, en su mayoría.

Dentro de las observaciones realizadas, aquella correspondiente al área de cerro (Observación 1, Tabla 3.9) fue la que presentó el mayor contenido de materia orgánica, de 10,4\%, con estructura granular. Estas áreas presentes en la microcuenca están cubiertas

por pastizales naturales, ya que, dado su escasa profundidad efectiva no son aptas para establecer cultivos agrícolas.

Por otro lado, se encontraron algunas áreas con síntomas de erosión, correspondientes a sectores de loma en cabeceras de lote con suelos con espesores del horizonte A menores 
que $20 \mathrm{~cm}$, con estructuras en bloques con tendencia a tipo laminar y contenidos de materia orgánica menores que $6 \%$.

En la Tabla 3.9 se presentan los valores del factor $\mathrm{K}$ calculados para las 12 observaciones realizadas en las unidades ambientales delimitadas en la microcuenca. Considerando dichas unidades, los valores resultaron entre 0,21 y 0,33 y el valor ponderado, según la superficie correspondiente, fue de 0,31 .

Tabla 3.9. K expresados en unidades métricas (Ton ha $\mathrm{h}$ (ha $\mathrm{MJ} \mathrm{mm})^{-1}$ ) obtenidos para las observaciones de suelos realizadas en las unidades ambientales delimitadas en la microcuenca. Valores del factor asignados a dichas unidades y superficie asociada. Factor $\mathrm{K}$ ponderado para la microcuenca bajo estudio.

\begin{tabular}{|c|c|c|c|c|c|}
\hline Unidad ambiental & Observación & $\begin{array}{l}\text { Factor } \mathrm{K} \\
\text { calculado }\end{array}$ & $\begin{array}{c}\text { Valor de K } \\
\text { asignado a la } \\
\text { unidad ambiental }\end{array}$ & $\begin{array}{l}\text { Superficie } \\
\text { de la } \\
\text { unidad }\end{array}$ & $\begin{array}{l}\text { Factor K } \\
\text { ponderado }\end{array}$ \\
\hline Cerros y pajonales & 1 & 0,21 & 0,21 & 53,6 & \multirow{12}{*}{0,31} \\
\hline \multirow{4}{*}{ Vaguadas } & 3 & 0,31 & \multirow{4}{*}{0,33} & \multirow{4}{*}{30,2} & \\
\hline & 8 & 0,33 & & & \\
\hline & 9 & 0,37 & & & \\
\hline & 12 & 0,34 & & & \\
\hline $\begin{array}{c}\text { Área cercana al } \\
\text { cauce }\end{array}$ & 5 & 0,28 & 0,28 & 24,7 & \\
\hline \multirow{2}{*}{$\begin{array}{l}\text { Área agrícola de } \\
\text { suelos someros }\end{array}$} & 2 & 0,29 & \multirow{2}{*}{0,3} & \multirow{2}{*}{119,0} & \\
\hline & 4 & 0,32 & & & \\
\hline \multirow{4}{*}{$\begin{array}{l}\text { Área agrícola de } \\
\text { suelos profundos }\end{array}$} & 6 & 0,24 & \multirow{4}{*}{0,33} & \multirow{4}{*}{339,0} & \\
\hline & 7 & 0,38 & & & \\
\hline & 10 & 0,36 & & & \\
\hline & 11 & 0,35 & & & \\
\hline
\end{tabular}

\subsubsection{Factor LS}

A partir de la información del modelo de elevación digital de la microcuenca (Figura 3.6), la misma se subdividió en área superior, media e inferior. Las mayores altitudes se encuentran en los sectores superior y medio de la microcuenca, con máximo de $285 \mathrm{msnm}$. Asimismo, la información del DEM fue empleada para la extracción de la red de drenaje (Figura 3.7) y para la elaboración del mapa de las pendientes de la microcuenca (Figura 3.8). El área superior posee la menor pendiente promedio, de 2,3\%, mientras que en el área media e 
inferior las pendientes medias son algo mayores, de 3,7 y 3,6\% respectivamente. Los datos de pendiente y de la red de drenaje se procesaron para obtener los valores correspondientes para el cálculo del factor LS. En la Tabla 3.10 se presentan estos resultados junto con los del factor LS considerando cada área delimitada en la microcuenca, y a su vez, la superficie asociada a pendientes menores y mayores que el $9 \%$. De este modo, se obtuvo un valor de LS ponderado de 0,45 .

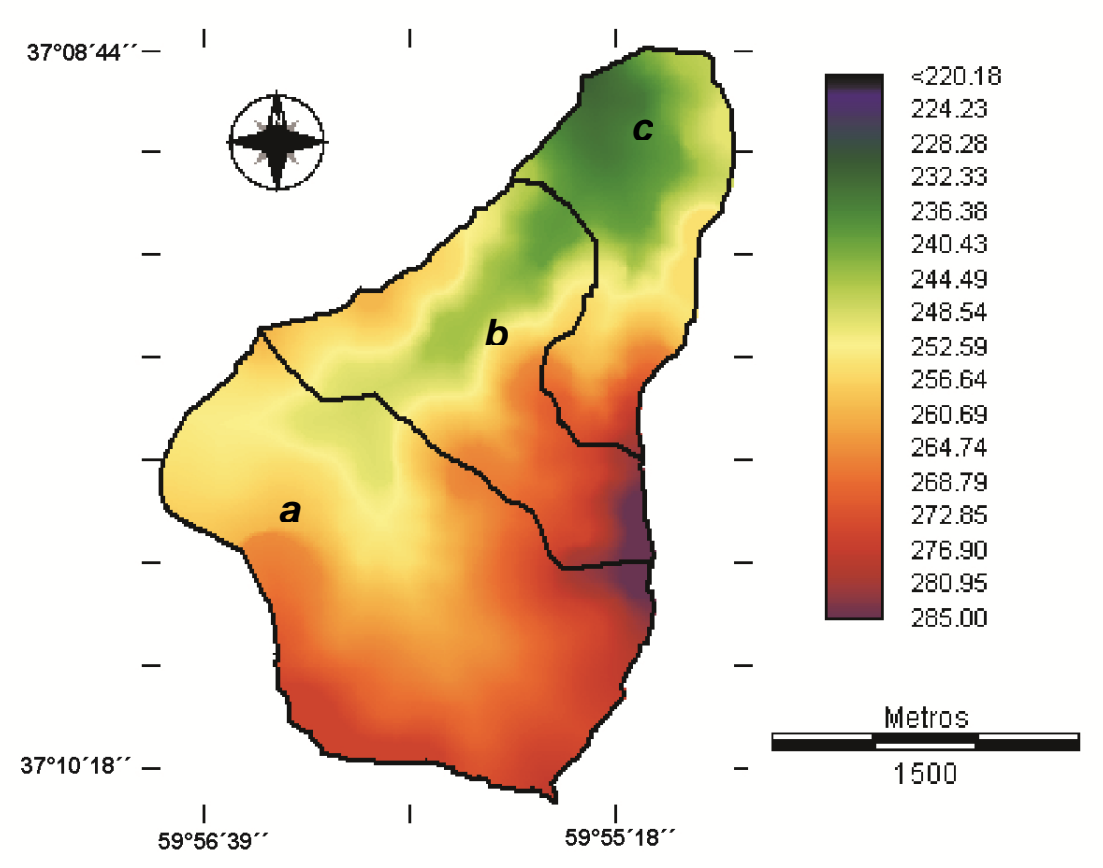

Figura 3.6. Modelo digital del terreno obtenido a partir de la digitalización de las curvas de nivel correspondientes a las cartas del Instituto Geográfico Militar, hojas 3760-22-1 "Base Azopardo" y 3760-22-3 "Chillar". Elevación expresada en metros sobre el nivel del mar. Áreas de subdivisión de la microcuenca de acuerdo con sus características físicas: a: sector superior; b: sector medio; c: sector inferior. 


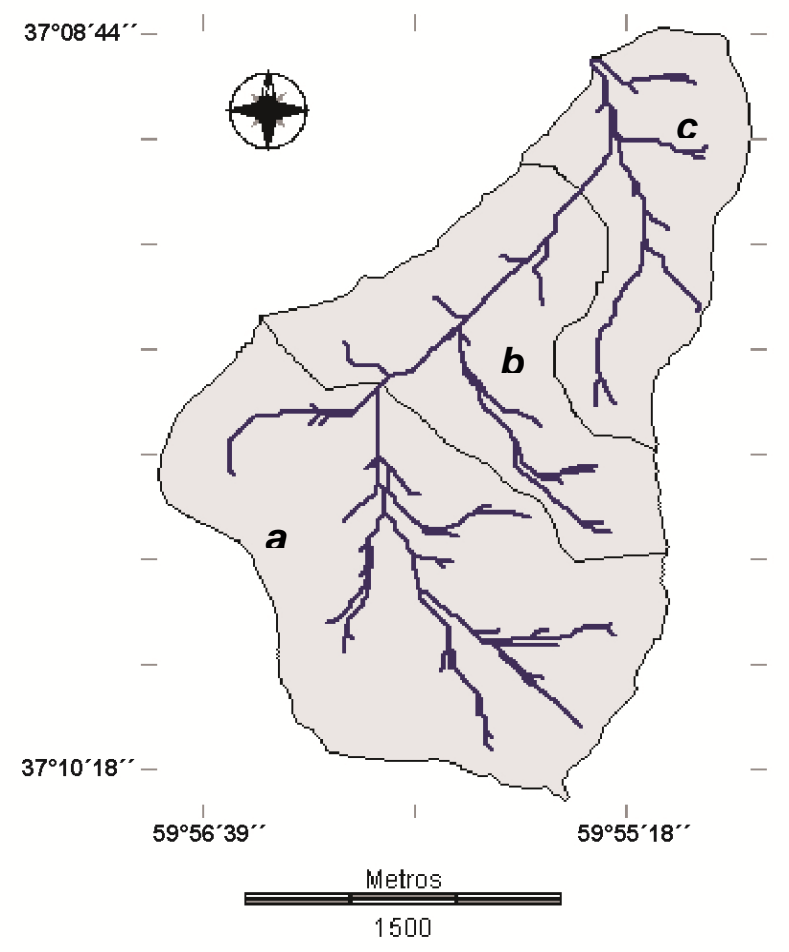

Figura 3.7. Red de drenaje de la microcuenca en sectores superior (a), medio (b) e inferior (c), a partir de la cual se calculó la longitud total de las vaguadas para obtener el factor LS.

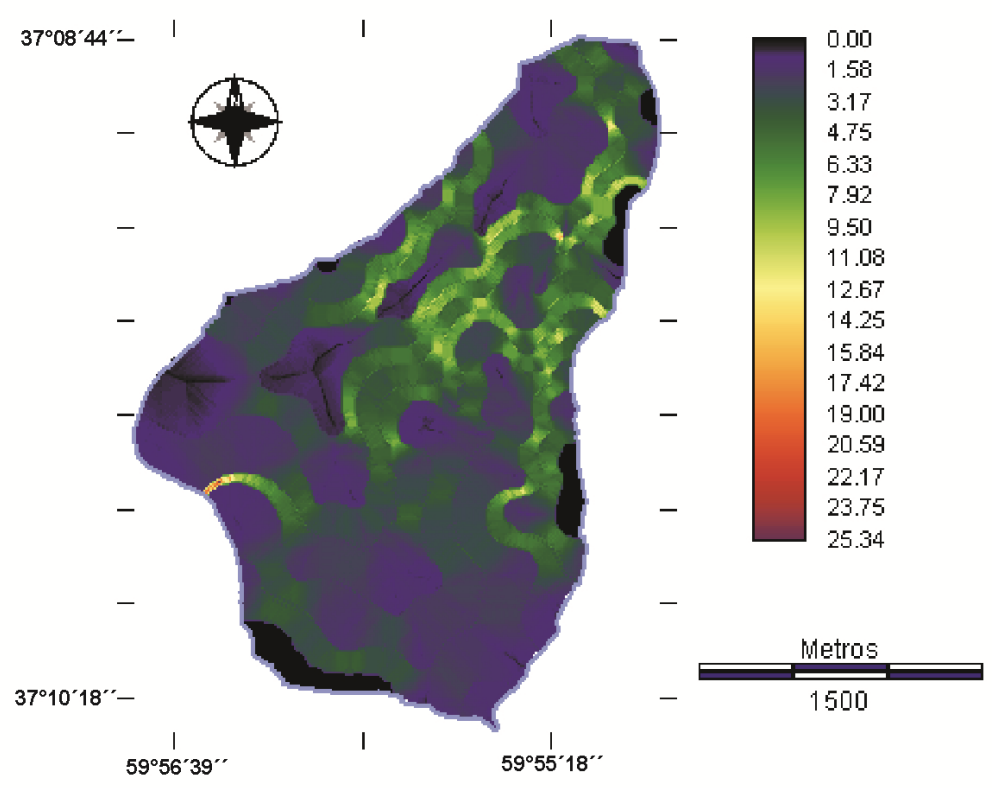

Figura 3.8. Mapa de pendientes correspondiente a la microcuenca bajo estudio. 
Tabla 3.10. Pendiente promedio, área, longitud de la pendiente $(\lambda)$ y valor de LS para las áreas de pendientes menores que $9 \%$ y mayores que $9 \%$ considerando sectores inferior, medio y superior de la microcuenca bajo estudio.

\begin{tabular}{|c|c|c|c|c|c|c|c|c|c|}
\hline \multirow[b]{2}{*}{ Parámetro } & \multicolumn{3}{|c|}{ Sector Inferior } & \multicolumn{3}{|c|}{ Sector Medio } & \multicolumn{3}{|c|}{ Sector Superior } \\
\hline & $\begin{array}{c}\text { Área de } \\
\text { pendiente } \\
\text { menor } 9 \%\end{array}$ & $\begin{array}{c}\text { Área de } \\
\text { pendiente } \\
\text { mayor } 9 \%\end{array}$ & $\lambda(\mathrm{m})$ & $\begin{array}{c}\text { Área de } \\
\text { pendiente } \\
\text { menor } 9 \%\end{array}$ & $\begin{array}{c}\text { Área de } \\
\text { pendiente } \\
\text { mayor } 9 \%\end{array}$ & $\lambda(\mathrm{m})$ & $\begin{array}{c}\text { Área de } \\
\text { pendiente } \\
\text { menor } 9 \%\end{array}$ & $\begin{array}{c}\text { Área de } \\
\text { pendiente } \\
\text { mayor } 9 \%\end{array}$ & $\lambda(\mathrm{m})$ \\
\hline $\begin{array}{c}\text { Pendiente } \\
\text { promedio } \\
(\%)\end{array}$ & 3,3 & 9,9 & \multirow{3}{*}{125,4} & 3,4 & 9,4 & \multirow{3}{*}{146,1} & 2,2 & 12,8 & \multirow{3}{*}{143,2} \\
\hline Área (ha) & 116,4 & 4,2 & & 149,8 & 5,6 & & 289,7 & 1,0 & \\
\hline Valor LS & 0,5 & 1,9 & & 0,5 & 1,9 & & 0,3 & 2,8 & \\
\hline
\end{tabular}

\subsubsection{Factor $\mathrm{C}$}

La superficie de la microcuenca se encuentra dividida en 7 lotes en los cuales, durante el período estudiado, se realizaron cultivos agrícolas. Asimismo, existen áreas no cultivables, correspondientes a cerros y pastizales naturales, que representan el $9,5 \%$ de la superficie.

Durante el período estudiado tuvieron lugar 5 ciclos de cultivos, 3 estivales y 2 invernales. En los ciclos estivales la superficie de la microcuenca estuvo mayormente cubierta por soja de primera y segunda siembra, que en promedio ocuparon $288 \mathrm{ha}$, en tanto que el maíz le siguió en importancia con 155 ha en promedio. El cultivo de girasol solo tuvo lugar en 34 ha durante el ciclo 2011-2012. El principal cultivo de invierno fue la cebada, con 146 ha en promedio, mientras que el trigo ocupó únicamente 27 ha en 2011.

En relación al cálculo del factor $\mathrm{C}$, en la Tabla 3.11 se presenta, a modo de ejemplo, la planilla elaborada para su cálculo correspondiente a 2 de los eventos considerados en este trabajo. La información de las restantes crecidas se muestra en la Tabla 1 del Anexo. Para cada cultivo, de acuerdo con la fecha del evento analizado, se identificó la etapa de crecimiento en la que se encontraba. Dichas etapas están definidas en el Manual 537 (Wischmeier \& Smith, 1978), donde se especifican los valores de relación de pérdida de suelo que les corresponden, según el cultivo y los antecesores de los que se trate. Se consideraron en este caso las etapas de:

-Siembra a $10 \%$ de cobertura del dosel;

-Período 1, desde la finalización de la etapa anterior hasta el 50\% de cobertura del dosel;

-Período 2, desde finalización de Período 1 hasta el 75\% de cobertura del dosel; 
-Período 3, entre finalización del período 2 y la cosecha de los cultivos;

-Período 4, correspondiente al barbecho, entre la cosecha y la siembra del próximo cultivo.

La Tabla 3.11 muestra una fecha coincidente con cultivos de invierno en el inicio de su ciclo y otra coincidente con cultivos de verano en sus últimas etapas. En ella, al igual que lo que muestra la Tabla 3.12, se observa que el valor de $C$ resultó más alto para eventos registrados entre el otoño y la primavera. En dicho período una parte importante de la microcuenca se encuentra ocupada por áreas en barbecho con rastrojos de cultivos de verano, los cuales, según lo que establece el Manual 537 (Wischmeier \& Smith, 1978), tienen valores de relación de pérdida de suelo que pueden alcanzar hasta el $40 \%$. En contraste, los residuos de cosecha de trigo o cebada muestran relación de pérdida de suelo menor, lo que sumado a la presencia de cultivos estivales desarrollados en su totalidad, determina que el valor de C ponderado sea cercano a $10 \%$ durante los eventos registrados en el verano.

Tabla 3.11. Planilla de cálculo del factor $C$, con detalle de fecha de la crecida, usos del suelo, etapa en la que se encontraban los cultivos y valor de relación de pérdida de suelo asociado para cada lote de la microcuenca. Valor de $\mathrm{C}$ ponderado para la crecida.

\begin{tabular}{|c|c|c|c|c|c|c|}
\hline $\begin{array}{l}\text { Fecha } \\
\text { evento }\end{array}$ & Lote & $\begin{array}{c}\text { Superficie } \\
\text { del lote (ha) }\end{array}$ & Uso del suelo & $\begin{array}{l}\text { Etapa del } \\
\text { cultivo }\end{array}$ & $\begin{array}{c}\text { Valor relación } \\
\text { pérdida de suelo }\end{array}$ & $\begin{array}{c}\text { Factor C } \\
\text { ponderado }\end{array}$ \\
\hline \multirow{8}{*}{$19 / 07 / 2011$} & 1 & 34,2 & Cebada & $\begin{array}{c}\text { Siembra- } 10 \% \\
\text { cobertura }\end{array}$ & 0,4 & \multirow{8}{*}{0,26} \\
\hline & 2 & 26,7 & Barbecho (rastrojo de soja $1^{a}$ ) & Período 4 & 0,4 & \\
\hline & 3 & 13,5 & Barbecho (rastrojo de soja $2^{\underline{a}}$ ) & Período 4 & 0,18 & \\
\hline & 4 & 90,4 & Barbecho (rastrojo de maíz) & Período 4 & 0,26 & \\
\hline & 5 & 180,1 & Barbecho (rastrojo de maíz) & Período 4 & 0,26 & \\
\hline & 6 & 59,9 & Cebada & $\begin{array}{c}\text { Siembra- } 10 \% \\
\text { cobertura }\end{array}$ & 0,31 & \\
\hline & 7 & 108,1 & Cebada & $\begin{array}{c}\text { Siembra- } 10 \% \\
\text { cobertura }\end{array}$ & 0,31 & \\
\hline & $\begin{array}{l}\text { Área cerros } \\
\text { y pajonales }\end{array}$ & 53,6 & - & & 0,00003 & \\
\hline \multirow{8}{*}{ 05/03/2012 } & 1 & 34,2 & Soja $2^{a}$ & Período 3 & 0,07 & \multirow{8}{*}{0,07} \\
\hline & 2 & 26,7 & Barbecho (rastrojo de trigo) & Período 4 & 0,08 & \\
\hline & 3 & 13,5 & Maíz & Período 3 & 0,11 & \\
\hline & 4 & 90,4 & Soja $1^{\mathrm{a}}$ & Período 3 & 0,07 & \\
\hline & 5 & 180,1 & Soja $1^{a}$ & Período 3 & 0,07 & \\
\hline & 6 & 59,9 & Barbecho (rastrojo de cebada) & Período 4 & 0,08 & \\
\hline & 7 & 108,1 & Soja $2^{\underline{a}}$ & Período 3 & 0,07 & \\
\hline & $\begin{array}{l}\text { Área cerros } \\
\text { y pajonales }\end{array}$ & 53,6 & - & & 0,00003 & \\
\hline
\end{tabular}


Tabla 3.12. Valores de C ponderados correspondientes a los 17 eventos analizados en la microcuenca bajo estudio.

\begin{tabular}{|c|c|}
\hline Fecha Evento & C ponderado \\
\hline $15 / 01 / 2011$ & 0,11 \\
\hline $01 / 05 / 2011$ & 0,20 \\
\hline $19 / 07 / 2011$ & 0,26 \\
\hline $08 / 11 / 2011$ & 0,14 \\
\hline $10 / 01 / 2012$ & 0,07 \\
\hline $05 / 03 / 2012$ & 0,07 \\
\hline $11 / 03 / 2012$ & 0,07 \\
\hline $18 / 04 / 2012$ & 0,09 \\
\hline $17 / 05 / 2012$ & 0,12 \\
\hline $23 / 08 / 2012$ & 0,15 \\
\hline $03 / 09 / 2012$ & 0,15 \\
\hline $05 / 10 / 2012$ & 0,14 \\
\hline $15 / 10 / 2012$ & 0,17 \\
\hline $22 / 11 / 2012$ & 0,13 \\
\hline $05 / 12 / 2012$ & 0,11 \\
\hline $19 / 12 / 2012$ & 0,10 \\
\hline $28 / 12 / 2012$ & 0,09 \\
\hline Promedio & 0,13 \\
\hline Coef Variación (\%) & 40,6 \\
\hline
\end{tabular}

\subsubsection{Factor $P$}

Dado que en la microcuenca no se aplican prácticas estructurales de control de la erosión hídrica, se asignó un valor del factor $\mathrm{P}=1$ para la totalidad de los lotes que la conforman.

\subsubsection{Valores MUSLE para cada evento}

En la Tabla 3.13 se presentan los valores calculados de pérdida de suelo obtenidos a través de la aplicación del modelo MUSLE. Los resultados se encontraron entre 3,6 y 418,6 toneladas, con un promedio de 100 toneladas y un coeficiente de variación que resultó mayor que 100\%. En 5 eventos se superó el promedio antes mencionado, correspondientes a las fechas $15 / 01 / 2011,17 / 05 / 2012,23 / 08 / 2012,05 / 12 / 2012$ y 19/12/2012. Para las crecidas registradas durante el año 2011 las pérdidas calculadas totalizaron 304,9 toneladas, mientras que aquellas producidas durante 2012 sumaron 1395 toneladas, es decir, un valor 4,6 veces mayor que el obtenido para 2011. 
Tabla 3.13. Valores de pérdida de suelo, expresados en toneladas, calculados mediante el modelo MUSLE para los 17 eventos analizados en la microcuenca bajo estudio.

\begin{tabular}{|c|c|}
\hline Fecha & $\begin{array}{c}\text { Valor MUSLE } \\
(\mathrm{t})\end{array}$ \\
\hline $15 / 01 / 2011$ & 254,9 \\
\hline $01 / 05 / 2011$ & 29,5 \\
\hline $19 / 07 / 2011$ & 12,6 \\
\hline $08 / 11 / 2011$ & 7,9 \\
\hline $10 / 01 / 2012$ & 5,9 \\
\hline 05/03/2012 & 6,8 \\
\hline $11 / 03 / 2012$ & 5,4 \\
\hline $18 / 04 / 2012$ & 3,6 \\
\hline $17 / 05 / 2012$ & 418,6 \\
\hline 23/08/2012 & 253,8 \\
\hline 03/09/2012 & 58,8 \\
\hline 05/10/2012 & 37,2 \\
\hline $15 / 10 / 2012$ & 89,3 \\
\hline $22 / 11 / 2012$ & 34,4 \\
\hline 05/12/2012 & 143,0 \\
\hline $19 / 12 / 2012$ & 279,2 \\
\hline $28 / 12 / 2012$ & 59,0 \\
\hline Promedio & 100,0 \\
\hline $\begin{array}{c}\text { Coef Variación } \\
(\%)\end{array}$ & 125,4 \\
\hline Mediana & 37,2 \\
\hline $\begin{array}{c}\text { Relación } \\
\text { promedio/mediana }\end{array}$ & 2,7 \\
\hline
\end{tabular}




\section{DISCUSIÓN}

\subsection{Precipitaciones, erosividad de las precipitaciones y reserva de agua en el suelo en el período estudiado}

En relación a las precipitaciones registradas durante los años estudiados según los resultados de la sección 3.1.1.b., las correspondientes a 2011 fueron inferiores al promedio anual de 40 años para la zona y las de 2012 superiores a dicho valor. Es decir, el período de análisis comprendió dos años contrastantes en este sentido.

De acuerdo con la tendencia de las lluvias, el valor anual calculado del índice $\mathrm{El}_{30}$ para el año 2012 fue superior al de 2011, y a su vez superaron al promedio de 13 años para la localidad de Azul. El análisis comparativo de estos valores considerando su distribución mensual indica que en 2011 la mayor proporción de la erosividad anual se concentró principalmente en un solo mes, enero, constituyendo el $63,3 \%$ del valor total para ese año. Por otro lado, las diferencias más importantes que se dieron en 2012 pueden asociarse a las precipitaciones de alta magnitud producidas durante los meses de mayo, agosto y diciembre, que ocasionaron eventos de crecidas importantes y con altas concentraciones de material en suspensión, como se analizará más adelante.

Asimismo, se registraron diferencias en la reserva de agua en el suelo entre ambos años estudiados (sección 3.2), y también en el comportamiento mensual de esta variable. De este modo, entre mayo y septiembre la reserva de agua en el suelo media diaria fue superior a $40 \mathrm{~mm}$ tanto para el año 2011 como para el 2012. En este período, comprendido entre el otoño y el inicio de la primavera, la evapotranspiración de referencia se mantiene en valores bajos, tal como fue analizado por Ares, (2010) a partir de datos correspondientes al período 2001-2007 de la estación Azul Aero del SMN (Figura 4.1). Esto determina que, aunque se registren precipitaciones de poca magnitud, la reserva de agua permanezca alta durante esos meses, lo que se reflejó en el coeficiente de variación, que resultó inferior a 40\%. Por otra parte, a lo largo de los meses restantes, en los que la evapotranspiración es mayor, la reserva de agua, en general, fue menor que $40 \mathrm{~mm}$. Sin embargo, su tendencia se relacionó con la que presentaron las precipitaciones en los años que fueron estudiados. Como ejemplo se puede mencionar lo ocurrido durante el mes de diciembre de 2011 para el cual, con lluvia mensual baja, la reserva resultó baja, en tanto que en diciembre de 2012, cuando la precipitación mensual fue alta, la reserva también lo fue (Figuras 3.2 y 3.4). 


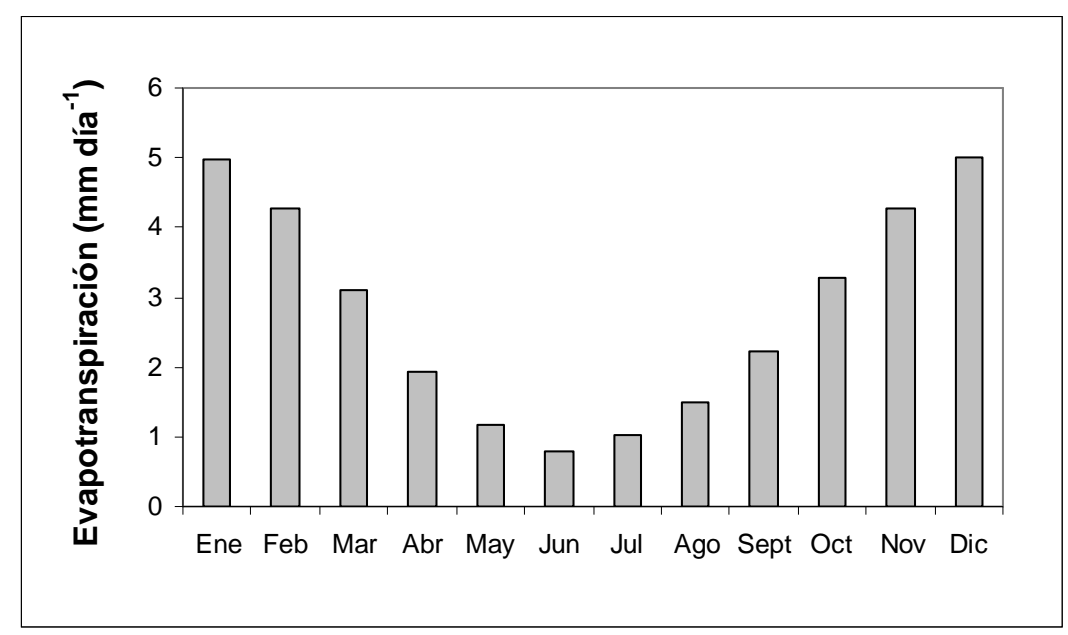

Figura 4.1. Evapotranspiración de referencia media mensual calculada para el período 20012007 correspondiente a la localidad de Azul (Fuente: Ares (2010)).

\subsection{Variabilidad en la respuesta a las precipitaciones y a la producción de sedimentos}

El análisis de los datos obtenidos (Tabla 3.1) indica la alta variabilidad en la cuenca en cuanto a la respuesta a las precipitaciones, ya que el escurrimiento total, el caudal pico, el caudal superficial medio y el coeficiente de escorrentía, tuvieron coeficientes de variación superiores a $100 \%$. Asimismo, la relación entre el promedio y la mediana para estas variables (Tabla 3.1) refleja la distribución sesgada y heterogénea que poseen. Esto se puede asociar a la variabilidad en la magnitud de las precipitaciones que originan las crecidas, como también a las diversas interacciones entre los factores que condicionan la respuesta hidrológica en cada evento, por ejemplo, la intensidad de las precipitaciones, el tiempo transcurrido desde la última precipitación y la evapotranspiración durante ese período que, junto a las propiedades edáficas, determinan la humedad del suelo (Pilgrim \& Cordery, 1993; Ponce \& Hawkins, 1996). A su vez, las características de la superficie en cuanto a su estado físico o a la cobertura de vegetación presente, influyen en la capacidad de infiltración del suelo, a través del efecto en la porosidad y el micro relieve. Estos factores poseen variabilidad espacial y temporal, y por lo tanto, conducen también a las diferencias en las respuestas al escurrimiento (Malet et al., 2003).

En cuanto a la producción de sedimentos, los resultados también evidencian la variabilidad registrada, la que fue más importante para el caso del muestreo discreto. Asimismo, la 
mayor concentración de sedimentos se produjo en pocos eventos y estos resultados coinciden con lo mencionado por Shipitalo \& Edwards (1998) González-Hidalgo et al. (2007) y Nu Fang et al. (2011), quienes señalan que la mayor cantidad de material suspendido se transporta en una cantidad reducida de tormentas.

\subsection{Relaciones entre las variables estudiadas}

En esta sección se discuten las relaciones entre las variables estudiadas en la presente tesis. Para una mejor organización, se consideran de manera separada las relaciones entre escurrimiento y precipitación, de aquellas entre la CSS, las variables del escurrimiento y las de la precipitación. A su vez, en cada uno de estos apartados se discuten los resultados a escala de eventos individuales y a escala estacional.

\subsubsection{Relaciones escurrimiento-precipitación}

\subsection{1.a. Análisis a escala de eventos individuales}

En términos generales, las variables asociadas al escurrimiento y a la precipitación se encontraron correlacionadas al considerar el conjunto de los eventos, lo que coincide con lo mencionado por diferentes autores acerca de estudios realizados en cuencas de otras regiones del mundo (Zabaleta et al., 2007; Oeurng et al., 2010; Rodríguez-Blanco et al., 2010; Nu-Fang et al., 2011). Por su parte, el CE tuvo relación únicamente con la I 30 , y en tal sentido Chow et al. (1994) mencionan la vinculación entre estas variables, aunque también establecen que el CE depende de la humedad antecedente del suelo, lo que no fue registrado en este caso.

Se analizaron las regresiones lineales simples entre el escurrimiento y la precipitación y entre el escurrimiento y el $\mathrm{El}_{30}$, que, de acuerdo a lo comentado anteriormente, fueron estadísticamente significativas $(p<0,05)$ (Figuras 4.2 y 4.3 ). Para el primer caso la precipitación explicó el $55 \%$ de la variabilidad, y en el segundo la relación presentó un coeficiente de determinación de $61 \%$. Estos resultados pueden ser importantes a la hora de predecir la salida de contaminantes disueltos de la microcuenca, por ejemplo, para lo cual sería posible emplear al índice $\mathrm{El}_{30}$ como un buen predictor del escurrimiento total. Sin embargo, tal como señala da Silva (2004), esta información resulta difícil de obtener porque suele ser limitada o no está disponible. En ese caso, de acuerdo con los resultados obtenidos, la precipitación sería la variable de medición sencilla que podría ser utilizada 
como predictora del escurrimiento total con buen ajuste, a partir de lluvias mínimas cercanas a $20 \mathrm{~mm}$, que fue el valor de lluvia mínimo que produjo escurrimientos durante el período estudiado.

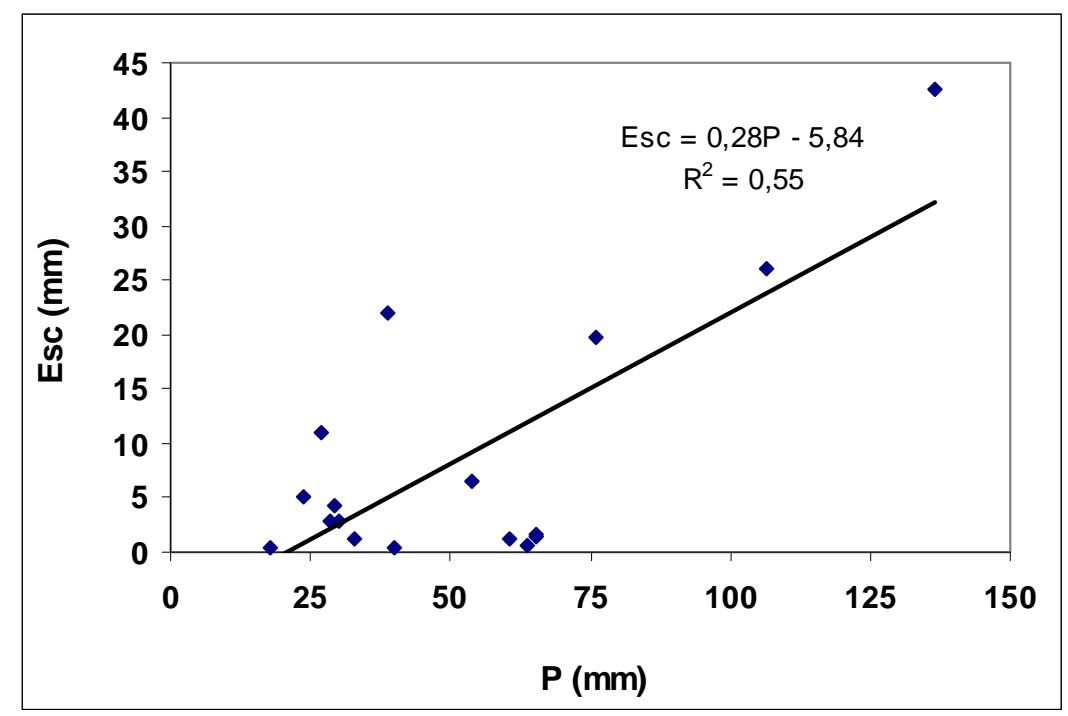

Figura 4.2. Relación entre el escurrimiento superficial y la precipitación total para los 17 eventos estudiados en la microcuenca del arroyo Videla.

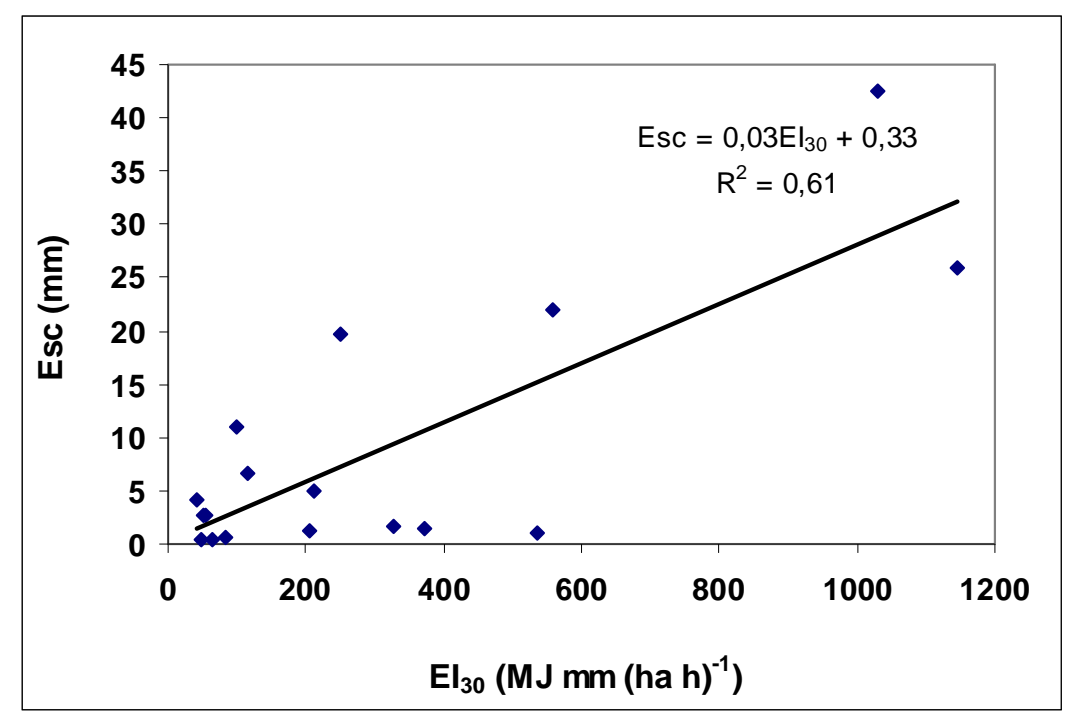

Figura 4.3. Relación entre el escurrimiento superficial y el índice $\mathrm{El}_{30}$ para los 17 eventos estudiados en la microcuenca del arroyo Videla.

En cuanto a la relación entre el CN y la precipitación, se graficaron los $\mathrm{CN}$ en función de las Iluvias a partir de los cuales fueron calculados. En esta relación secundaria que surge entre estas variables, en la mayoría de los casos, los CN calculados se aproximan a un valor 
asintótico con el incremento de las precipitaciones. Dicho valor es el CN que describe al conjunto de los datos para los eventos de lluvias mayores (Hawkins, 1993) y al funcionamiento de la cuenca, por lo que es una herramienta valiosa en el análisis de eventos de crecidas (Van Mullem et al., 2002). En la Figura 4.4 se presenta la relación obtenida, en la que se pudo advertir que los $C N$ se estabilizaban en valores cercanos a 62 asociados a lluvias de alta magnitud diaria. Si bien la cantidad de datos hasta el presente no ha permitido realizar un ajuste matemático, el registro de un escurrimiento correspondiente a una lluvia de aproximadamente $140 \mathrm{~mm}$, resultó significativo para caracterizar la respuesta hidrológica de dicha microcuenca. En la misma figura se observa que ante lluvias de milimetraje intermedio, los valores de $\mathrm{CN}$ resultaban comparativamente más bajos, cercanos a 50, aunque con una mayor dispersión. Finalmente, dicha microcuenca generó altos valores de $\mathrm{CN}$ asociados a lluvias de escasa cuantía diaria pero en condiciones de alta humedad antecedente, o bien relacionados a lluvias de alta intensidad, que pueden generar alto escurrimiento, de acuerdo a lo que establece Hjelmfelt (1991). Esto se puede verificar al analizar casos en los cuales los CN fueron superiores a 80 (Tabla 3.1). Por ejemplo, el evento correspondiente al 03/09/2012 con un CN de 82 registró un valor de reserva de agua en el suelo en el día anterior a la precipitación de 85,7 mm. Aquel registrado el 19/12/2012, con CN de 93 presentó lluvia intensa, con valores de $\mathrm{I}_{30}$ de $57,2 \mathrm{~mm} \mathrm{~h}^{-1}$ y de $\mathrm{I}_{10}$ de $68,4 \mathrm{~mm}$ $\mathrm{h}^{-1}$, en tanto que en la crecida del 28/12/2012 coincidieron condiciones de alta humedad antecedente (90 $\mathrm{mm}$ de reserva de agua en el suelo en el día anterior a la precipitación) y altas intensidades de lluvia $\left(\mathrm{I}_{30}\right.$ de $36,8 \mathrm{~mm} \mathrm{~h}^{-1}$ e $\mathrm{I}_{10}$ de $\left.75,6 \mathrm{~mm} \mathrm{~h}^{-1}\right)$

Estudios previos realizados en la cuenca del arroyo Videla (Ares et al., 2012b), demostraron un comportamiento similar al de la microcuenca con valores de $\mathrm{CN}$ que tienden a 57 $\left(R^{2}=0,79\right)$ ante lluvias de alta pluviometría diaria a partir de la siguiente función exponencial

$$
C N(P)=45,5 * e^{(-0,034 * P)}+56,9
$$

De este modo, la forma de la respuesta, tanto de la microcuenca como de la cuenca de arroyo Videla, sigue el patrón más frecuente al cual Hawkins (1993) lo define como "estándar". Los valores de equilibrio de la microcuenca resultaron ligeramente más altos que los de la cuenca lo cual coincide con lo observado por diversos autores como Esteves \& Lapetite (2003), Cerdan et al. (2004) y Feng \& Li (2008). Dichos autores hallaron que en cuencas de mayor tamaño relativo, se produce una reducción de la lámina de escorrentía atribuible al mayor tiempo medio en el que este se inicia como también a la importante variabilidad espacial en cuanto al almacenamiento en depresiones, la cobertura vegetal y las 
capacidades de infiltración diferenciales de los suelos, los que actuarían regulando el flujo superficial. Esto se relacionaría con el valor de CN mayor al que tiende la microcuenca, en la que los tiempos de respuesta hidrológica serían más breves.

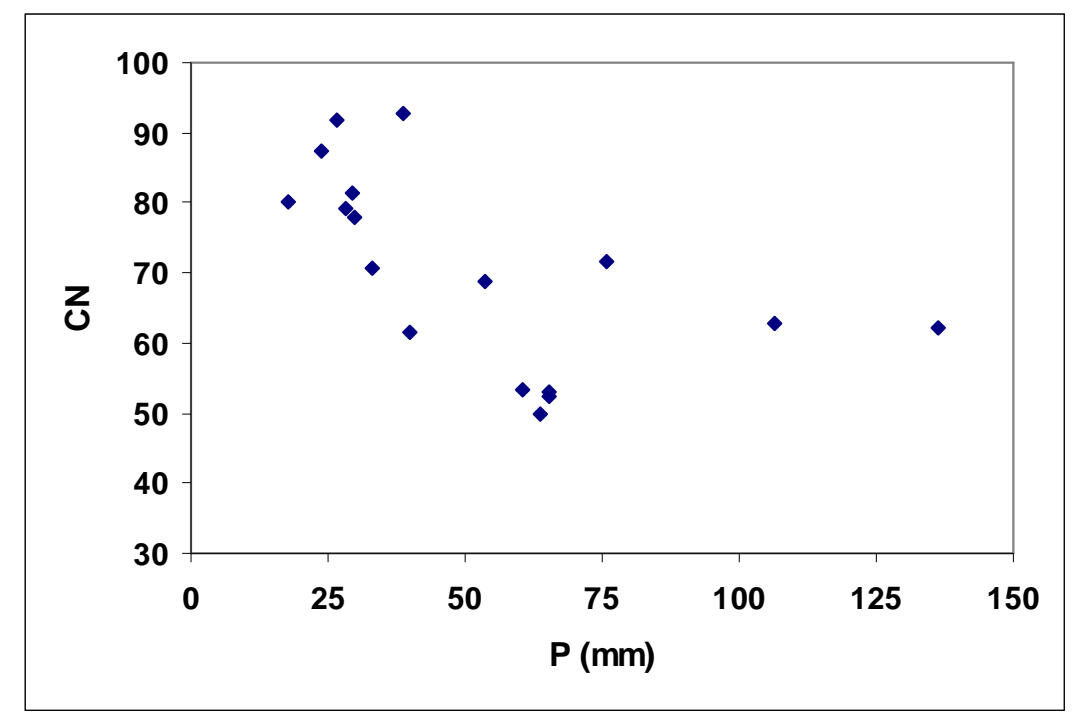

Figura 4.4. Relación entre los valores de CN calculados en función de la lámina de agua precipitada para los 17 eventos registrados en la microcuenca bajo estudio.

\subsection{1.b. Análisis estacional: períodos de baja a media cobertura y de alta cobertura}

Ciertas relaciones entre variables mostraron significancia estadística cuando fueron considerados todos los eventos registrados. Sin embargo, al agrupar a los casos estacionalmente, en algunos de ellos, las mismas relaciones presentaron significancia únicamente para uno de los 2 períodos considerados. Esto se evidenció, por ejemplo, en la relación escurrimiento-precipitación, la que presentó correlación significativa para el conjunto de los eventos, mientras que en el análisis estacional tal significancia se manifestó en el período de media a baja cobertura. Esta tendencia muestra que el agrupamiento estacional puede contribuir a describir con más detalle los procesos involucrados en la generación de escurrimiento y producción de sedimentos, ya que los factores que controlan dichos procesos, como las lluvias y la cobertura vegetal ejercen una acción diferencial vinculada a su dinámica temporal (Smith, 2008; Estrany et al., 2009).

Así, detallando el análisis de las relaciones entre escurrimiento y precipitación, las correlaciones de Pearson mostraron distintas asociaciones entre las variables de la precipitación y las del escurrimiento al considerar períodos de media a baja cobertura y de 
alta cobertura, como fue descripto en la sección 3.3.1.b. En el primer caso se registraron relaciones significativas entre Esc, Qsm y P, Dur, E, $\mathrm{El}_{30}$ e $\mathrm{I}_{10}$ en tanto que el Qp se relacionó con las tres primeras variables de la precipitación antes enumeradas. En cuanto al período de alta cobertura, Esc, Qp y Qsm demostraron correlaciones significativas con la $I_{30}$, mientras que únicamente el Esc se relacionó con $\mathrm{El}_{30}$. Tales resultados podrían mostrar diferencias en los mecanismos de generación de escurrimiento que fueron predominantes en la microcuenca en ambos períodos, los que podrían ser asociados a las características de las precipitaciones en cada caso. Por ejemplo, la relación entre las variables del escurrimiento y la intensidad máxima de la lluvia en 30 minutos podría ser indicativa de preponderancia de flujo superficial hortoniano como mecanismo de generación de escurrimiento en el período de alta cobertura. Dicho mecanismo se produce en áreas en las que la intensidad de la lluvia supera la capacidad de infiltración de los suelos (Pilgrim \& Cordery, 1993). La bibliografía menciona que su ocurrencia no sería relevante en áreas húmedas y con cobertura vegetal (Dunne et al., 1975), y en ese sentido, Sidle et al. (2007) lo encuentran como un componente menor del escurrimiento en cuencas forestadas. En la microcuenca bajo estudio este tipo de flujo podría estar asociado a algunos factores que se dan de manera conjunta. En primer lugar, el período de alta cobertura este período coincide con la primavera, el verano y parte del otoño, cuando las precipitaciones tienen mayor intensidad. En la Tabla 4.1. se muestra la intensidad promedio de las lluvias registradas en este caso. Asimismo, durante esta etapa, la evapotranspiración es alta, lo que conduce a mantener los niveles freáticos más bajos (Varni \& Custodio, 2013b) y al suelo con menor reserva de agua, de acuerdo a lo analizado en la sección 4.1. Por su parte, la presencia de suelos con profundidad limitada podría ser otra condición favorable para la generación del escurrimiento, dado que el suelo se satura rápidamente. Además, de acuerdo a lo que menciona la bibliografía, el escurrimiento superficial confluye en determinados sectores condicionado por la topografía y puede producir la formación de surcos, los que fueron observados en el campo posteriormente a algunos de estos eventos (Sidle et al., 2007).

En cuanto al período de media a baja cobertura, las relaciones entre las variables del escurrimiento y la precipitación indicarían que el flujo superficial hortoniano podría ser uno de los mecanismos de generación del escurrimiento, ya que la $l_{10}$ mostró significancia estadística en tal sentido. Es posible que ese flujo haya tenido relevancia en tormentas de alta intensidad, como la registrada el 17/05/2012. Al mismo tiempo, mecanismos de flujo subsuperficial e infiltración desde superficie podrían producir saturación en el suelo. Este proceso, conocido como generación de escurrimiento en áreas parciales de saturación, podría tener lugar en estas circunstancias, lo cual explicaría las correlaciones que se presentaron entre Ptot, Dur, E y variables del escurrimiento. Dichos flujos son característicos 
de áreas húmedas con suelo cuya capacidad de infiltración supera la intensidad de las lluvias, con excepción de las lluvias extremas (Chow et al., 1994; Dunne et al., 1975). Este período coincide con parte del otoño, el invierno y el inicio de la primavera, y algunas condiciones que se dan durante el mismo pueden favorecer estos mecanismos. Por ejemplo, las precipitaciones registradas fueron de menor intensidad en promedio (Tabla 4.1) y esto habría favorecido la infiltración. En tanto, la evapotranspiración es menor, lo que se asocia a la escasa actividad vegetal predominante y a las menores temperaturas del aire. Así, la reserva de agua en el suelo resulta mayor, tal como mostraron los datos analizados en este trabajo (Figura 3.4), al igual que los niveles freáticos de la región son más elevados (Varni \& Custodio, 2013b). De tal modo, en este caso, la humedad antecedente tendría vinculación con la generación de escurrimiento superficial. Si bien la reserva de agua en el suelo no se correlacionó con la lámina escurrida al ser considerados todos los eventos en su conjunto (Tabla 3.2), se analizó su relación para estos 5 casos asociados al período de baja cobertura. La Figura 4.5 muestra la relación resultante, para la que se ajustó una función exponencial. Aunque la cantidad de datos para el estudio de este período pueda resultar baja, el $\mathrm{R}^{2}$ indica que la ResA logró explicar el $51 \%$ de la variabilidad en el Esc. Esto contribuiría a establecer que también durante este período el transporte de agua hacia el curso podría haberse producido por condiciones de saturación del suelo desde su parte interna a partir de la generación de flujos tanto superficial como subsuperficial. Cabe comentar que en esta relación se observa un punto alejado de la tendencia de los datos, el que corresponde al evento del 17/05/2012, que presentó alta intensidad de precipitación y que generó formación de surcos en el campo, por lo cual es posible que la intensidad de la precipitación haya sido el factor de mayor influencia en su respuesta al escurrimiento.

Por último, diferencias estacionales en cuanto a los mecanismos que producen el escurrimiento han sido reconocidas por Giménez et al., (2012) en cuencas del Mediterráneo.

Tabla 4.1. Intensidad promedio de las precipitaciones en 10 y 30 minutos correspondiente a la totalidad de las tormentas registradas en el período de media a baja cobertura $(n=11)$ y en el período de alta cobertura $(n=33)$.

\begin{tabular}{|c|c|c|}
\hline Período & $\begin{array}{c}\mathrm{I}_{10} \text { promedio } \\
\left(\mathrm{mm} \mathrm{h}^{-1}\right)\end{array}$ & $\begin{array}{c}\mathrm{I}_{30} \text { promedio } \\
\left(\mathrm{mm} \mathrm{h}^{-1}\right)\end{array}$ \\
\hline $\begin{array}{c}\text { Media-Baja } \\
\text { cobertura }\end{array}$ & 25,2 & 16 \\
\hline Alta cobertura & 38,9 & 23,3 \\
\hline
\end{tabular}




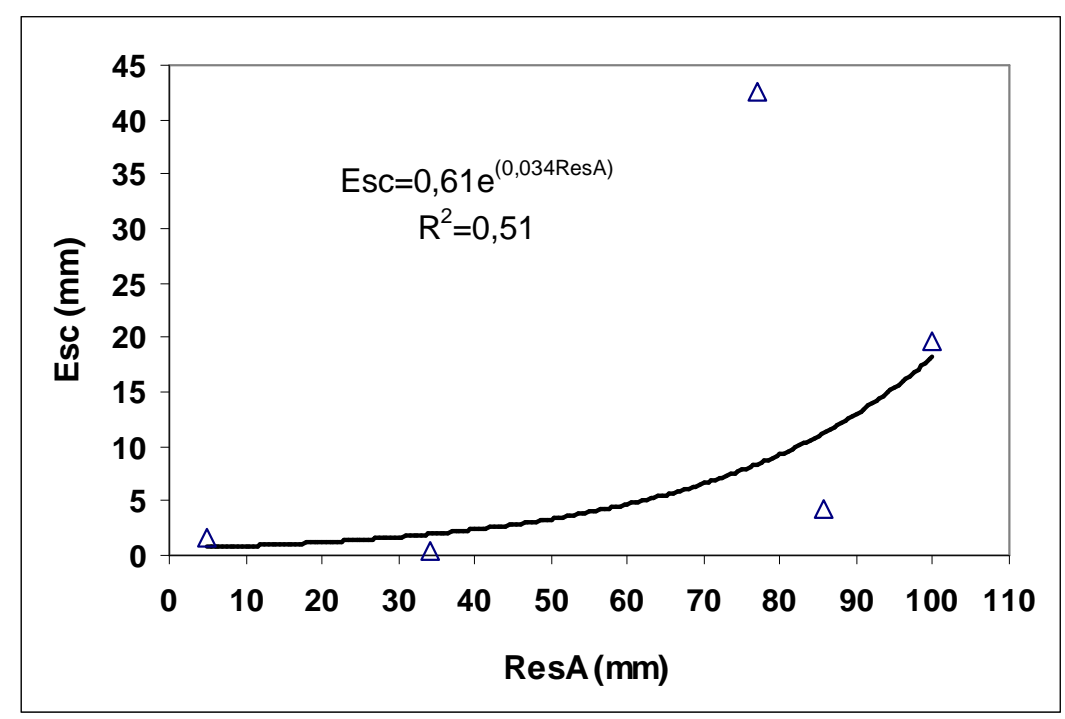

Figura 4.5. Relación entre el escurrimiento y la reserva de agua en el suelo del día previo a la precipitación para el período de media a baja cobertura $(n=5)$.

Acerca de los comentarios anteriores se presentan algunas figuras que muestran las relaciones entre variables del escurrimiento y la precipitación para los períodos diferenciados (Figuras 4.6, 4.7, 4.8 y 4.9). A partir de su comparación se pueden mencionar las diferencias asociadas a la variabilidad estacional en las respuestas al escurrimiento. Así, la dispersión fue mayor en el período de alta cobertura, mientras que fue menor con media a baja cobertura, lo que se refleja en los coeficientes de determinación de las relaciones analizadas. De acuerdo con esto, Smith (2008) y Estrany et al. (2009) hacen referencia a diferencias estacionales en la respuesta hidrológica vinculadas al crecimiento de la vegetación, la humedad en el suelo y las características de las lluvias. En tal sentido, durante la etapa de media a baja cobertura la respuesta hidrológica se vería favorecida por el mínimo crecimiento de los cultivos, ya que en este caso el área de estudio estaba principalmente cubierta por rastrojos, lo que implicaba homogeneidad en este sentido. Además, el estado de humedad era alto y la evaporación menor, como fuera descripto antes. Todo esto se habría reflejado en el menor coeficiente de variación obtenido en la reserva de agua en el suelo (Figura 3.4), y asimismo, en la menor dispersión en la respuesta al escurrimiento. Por el contrario, en el período de alta cobertura la microcuenca se encontraba ocupada por cultivos en crecimiento activo y por rastrojos, por lo que había mayor heterogeneidad en cuanto a las condiciones de la cobertura. Se considera que esto contribuyó a la variabilidad en la reserva de agua en el suelo, que fue mayor (Figura 3.4), sumado a la mayor evapotranspiración y a las características de las precipitaciones, en cuanto a su magnitud e intensidad. De este modo, la dispersión en la respuesta podría ser relacionada a las condiciones detalladas. 


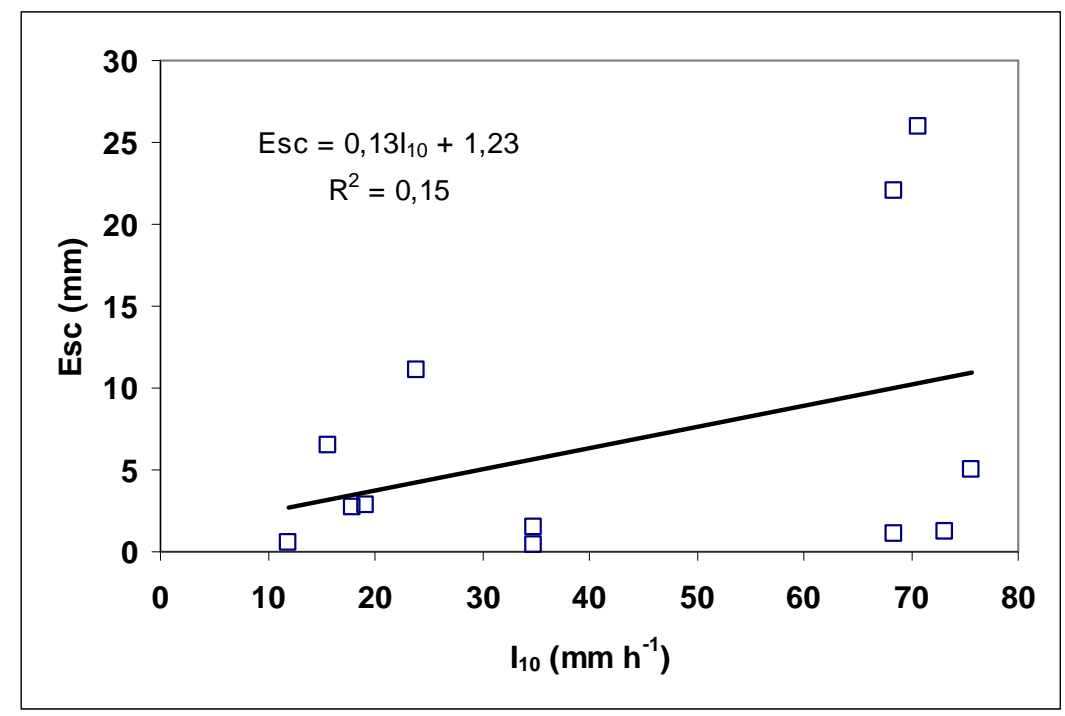

Figura 4.6. Relación entre el escurrimiento y la intensidad máxima de la precipitación en 10 minutos, considerando los casos correspondientes al período de alta cobertura.

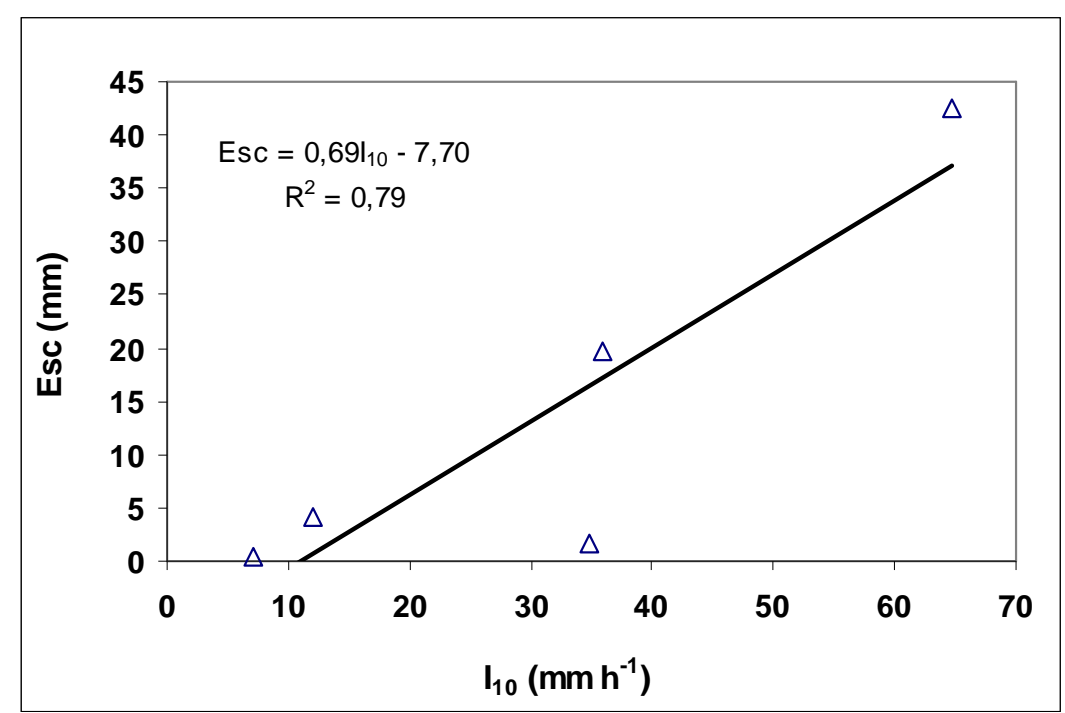

Figura 4.7. Relación entre el escurrimiento y la intensidad máxima de la precipitación en 10 minutos, considerando los casos correspondientes al período de media a baja cobertura. 


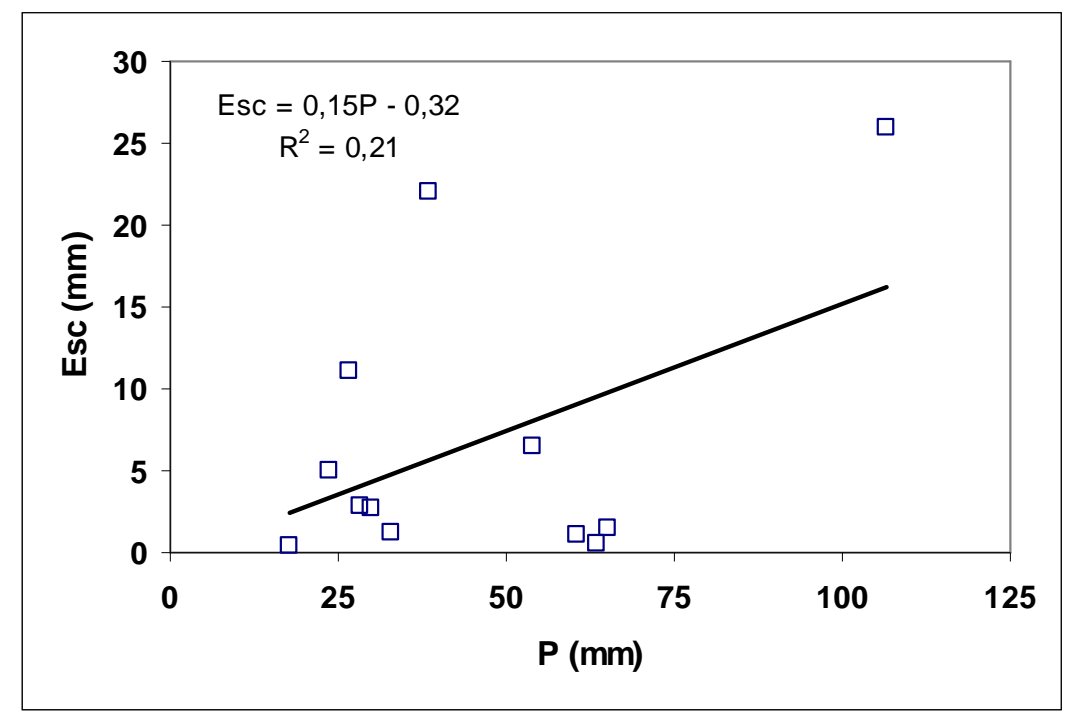

Figura 4.8. Relación entre el escurrimiento y la precipitación considerando los casos correspondientes al período alta cobertura.

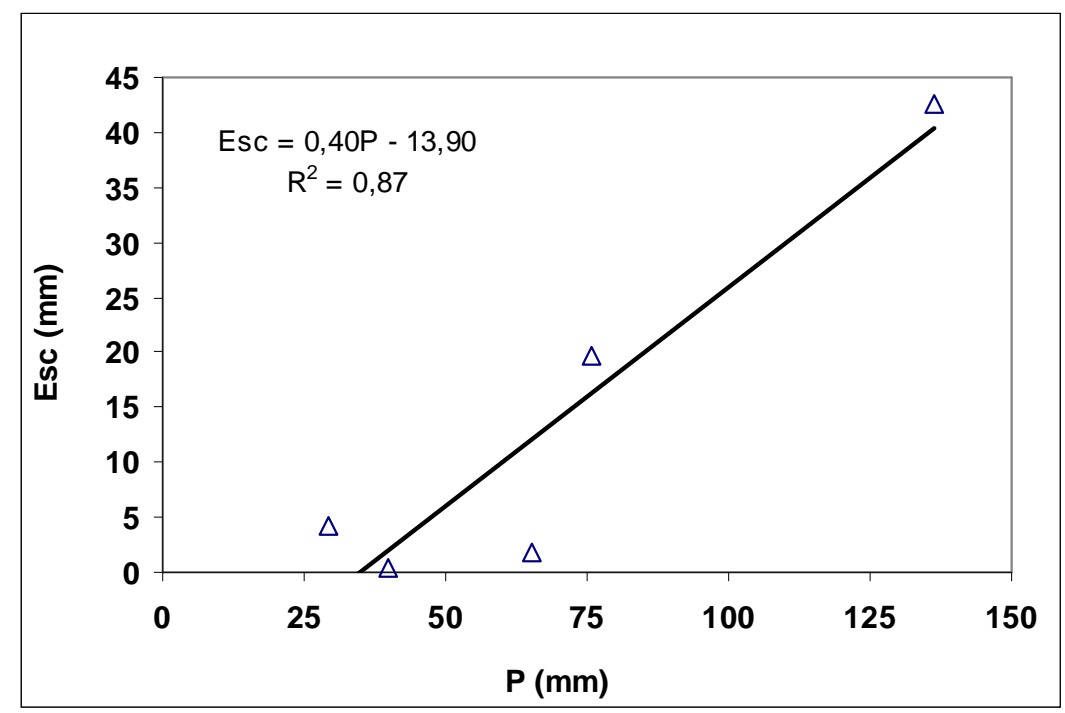

Figura 4.9. Relación entre el escurrimiento y la precipitación considerando los casos correspondientes al período de media a baja cobertura.

\subsubsection{Relaciones entre CSS, variables del escurrimiento y variables de la precipitación}

\subsection{2.a. Caracterización de los muestreos discreto y compuesto}

De acuerdo a lo mencionado en la sección 2.5, la recolección de agua de escurrimiento inició a una altura fija (0,3 m), lo que permitió obtener muestras durante el año 2011, en que las precipitaciones fueron menores y la mayor parte de los eventos, por lo tanto, de menor 
envergadura. Al mismo tiempo, esto determinó que entre los diferentes eventos, y según la magnitud de las crecidas y el escurrimiento pico que alcanzaron, la recolección discreta se produjera alrededor del máximo escurrimiento en eventos menores, mientras que en aquellas de mayor magnitud se produjera en la fase ascendente de la crecida. De igual modo, el muestreo compuesto, al extenderse durante 1,5 horas, tuvo lugar entre el pico de escurrimiento y su descenso o bien entre la fase ascendente y el escurrimiento máximo. Los eventos asociados a cada caso mencionado se detallan en la Tabla 4.2, y en las Figuras 4.10 y 4.11 se presentan dos eventos en los que se señalan los momentos de muestreo para ejemplificar lo mencionado antes.

Tabla 4.2. Momentos de muestreo discreto y compuesto, en relación a la magnitud de la curva de escurrimiento, y eventos asociados a cada caso.

\begin{tabular}{|c|c|c|}
\hline $\begin{array}{l}\text { Momento de } \\
\text { muestro en } \\
\text { relación a curva } \\
\text { de escurrimento }\end{array}$ & $\mathrm{N}^{\circ}$ Evento & Fecha \\
\hline \multirow{6}{*}{$\begin{array}{l}\text { Entre el pico de } \\
\text { escurrimiento y } \\
\text { su descenso }\end{array}$} & 3 & $19 / 07 / 2011$ \\
\hline & 4 & $08 / 11 / 2011$ \\
\hline & 5 & $10 / 01 / 2012$ \\
\hline & 6 & $05 / 03 / 2012$ \\
\hline & 7 & $11 / 03 / 2012$ \\
\hline & 8 & $18 / 04 / 2012$ \\
\hline \multirow{11}{*}{$\begin{array}{l}\text { Entre inicio y pico } \\
\text { de escurrimiento }\end{array}$} & 1 & $15 / 01 / 2012$ \\
\hline & 2 & $01 / 05 / 2011$ \\
\hline & 9 & $17 / 05 / 2012$ \\
\hline & 10 & $23 / 08 / 2012$ \\
\hline & 11 & 03/09/2012 \\
\hline & 12 & 05/10/2012 \\
\hline & 13 & $15 / 10 / 2012$ \\
\hline & 14 & $22 / 11 / 2012$ \\
\hline & 15 & 05/12/2012 \\
\hline & 16 & $19 / 12 / 2012$ \\
\hline & 17 & 28/12/2012 \\
\hline
\end{tabular}




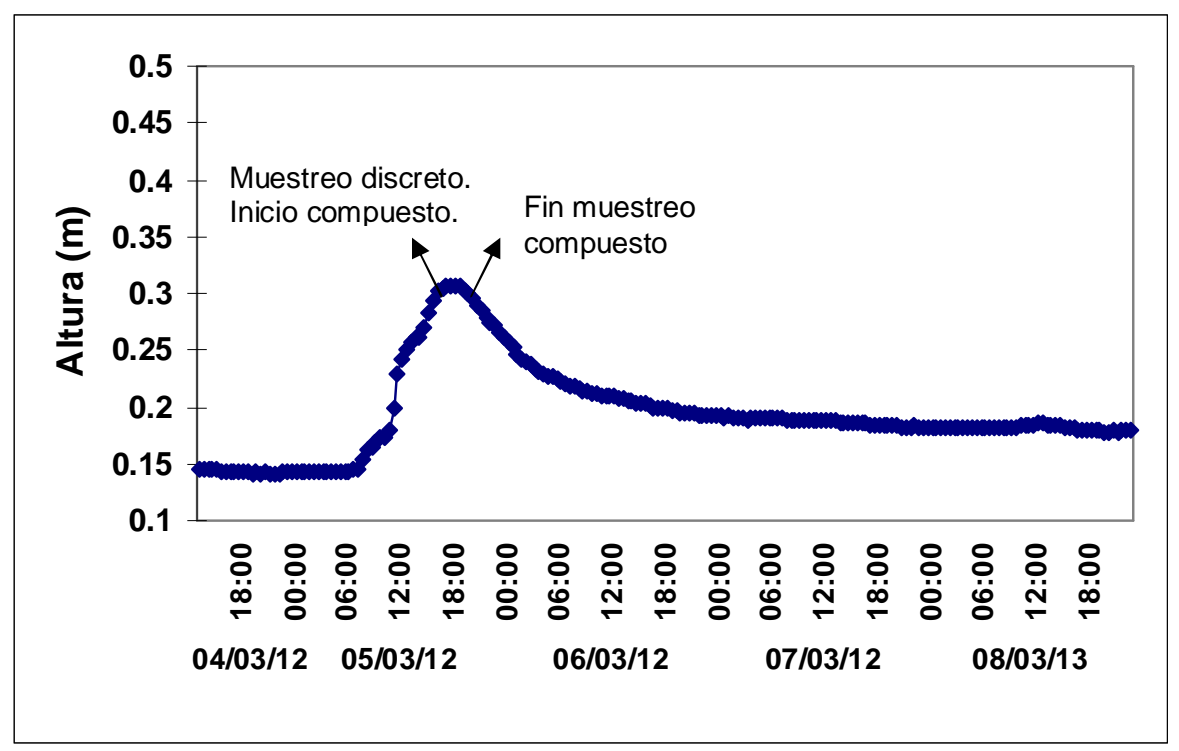

Figura 4.10. Alturas correspondientes al evento registrado el 05/03/2012. Momentos de recolección de muestras, en este caso, entre el pico de escurrimiento y su descenso.

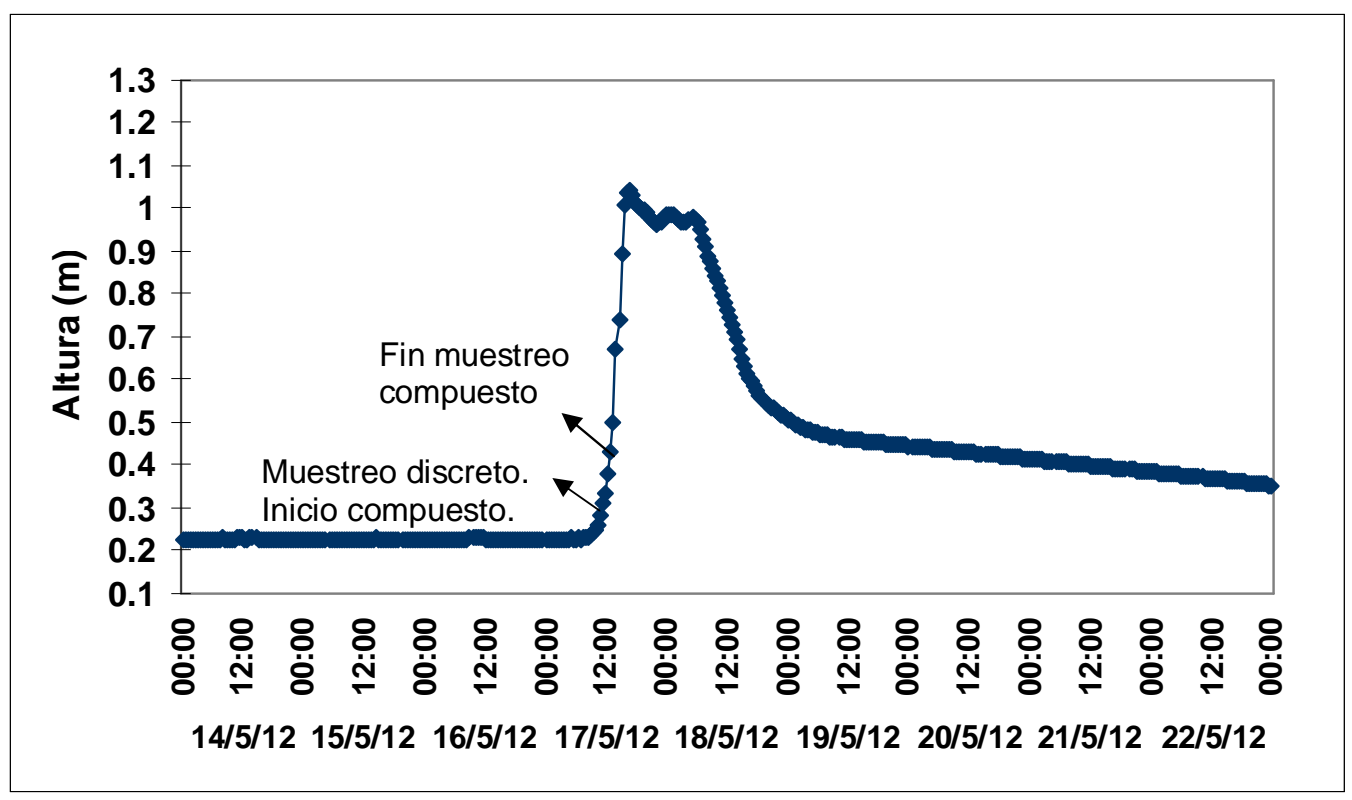

Figura 4.11. Alturas correspondientes al evento registrado el 17/05/2012. Momentos de recolección de muestras, en este caso, en la fase de ascenso de escurrimiento. 


\subsection{2.b. Análisis a escala de eventos individuales}

En este caso, se consideran las relaciones entre la concentración de ambos muestreos realizados y las variables asociadas al escurrimiento y la precipitación.

En primer lugar, el análisis de regresión lineal simple entre la concentración de sedimentos suspendidos correspondiente al muestreo discreto y el caudal superficial medio de cada evento (Figura 4.12) muestra que este último factor logró explicar el $69 \%$ de la variabilidad en cuanto a la concentración de sedimentos suspendidos $(p<0,05)$. Si bien esta relación presentó un elevado coeficiente de determinación, la mayor dispersión estaría dada a partir de valores de caudal superficial medio superiores a $0,5 \mathrm{~m}^{3} \mathrm{~s}^{-1}$ aproximadamente. Al mismo tiempo, las relaciones entre la CSS discreta y el Esc, Qp y CE mostraron una tendencia comparable a la comentada antes, pero con mayor dispersión, lo que se reflejó en un valor de $\mathrm{R}^{2}$ menor de las ecuaciones de regresión lineal simple (Tabla 4.3). Estos resultados confirman la presunción que el proceso erosivo en la microcuenca posee un comportamiento no lineal de alta variabilidad temporal en concordancia con lo mencionado por diversos autores (Gallart et al., 2002, Soler et al., 2008, López-Tarazón et al., 2010 y RodríguezBlanco et al., 2010). Según Seeger et al. (2004) y Nu-Fang et al. (2011) esta variabilidad en las concentraciones es consecuencia de las distintas interacciones que se dan en cada evento entre los factores involucrados en la respuesta hidrológica. Aquí entrarían en juego las características de las precipitaciones, su época de ocurrencia que puede condicionar la intensidad y la erosividad; y las condiciones de la cobertura vegetal o la ocurrencia de eventos previos que determinarían la disponibilidad de material para erosionarse. Así, se pueden presentar como ejemplo las relaciones entre la CSS disc y las variables $I_{30}, I_{10}$ y $E_{30}$. que se vincularon estadísticamente de manera significativa (Figuras 4.13, 4.14 y 4.15 ). Esto manifiesta que la variabilidad en la concentración podría ser explicada, en cierta medida, por la variabilidad en las características de las lluvias.

Por su parte, el factor de escurrimiento de la MUSLE también se encontró correlacionado con la CSS discreta, aunque la relación igualmente presenta dispersión a partir de valores del factor cercanos a 5.000 (Figura 4.16). Esto indica que este factor daría estimaciones aproximadas de las concentraciones puntuales por debajo de valores de $2 \mathrm{~g} \mathrm{l}^{-1}$, mientras que la incertidumbre sería mayor al considerar valores superiores. 


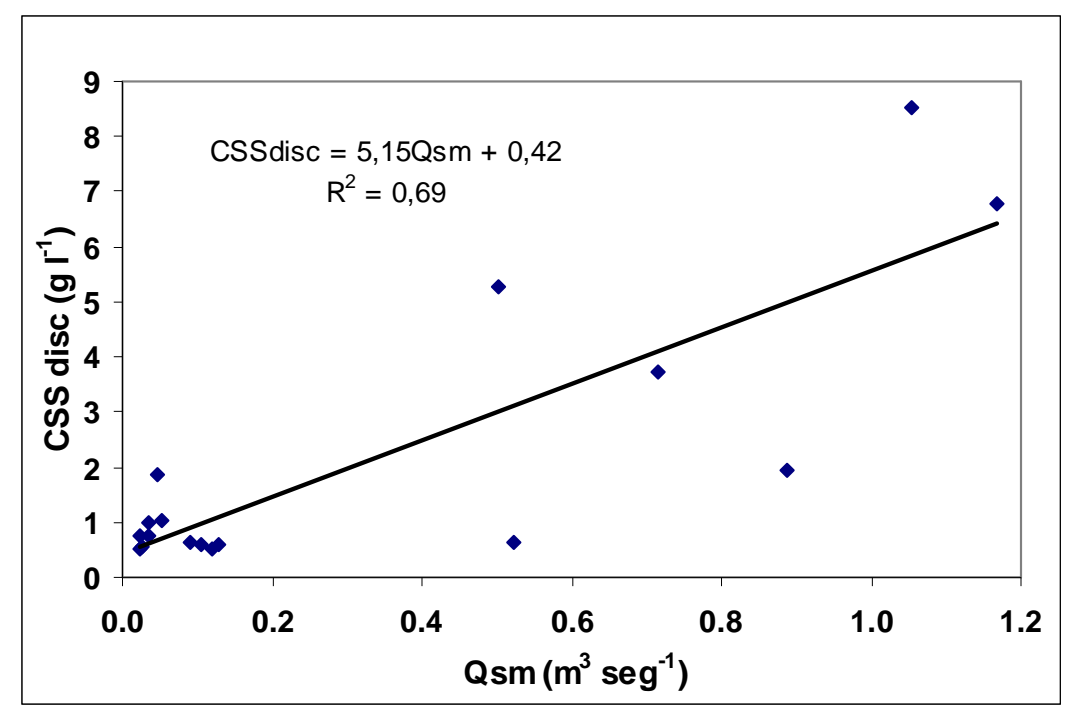

Figura 4.12. Relación entre la concentración de sólidos suspendidos del muestreo discreto y el caudal superficial medio para los 17 eventos estudiados en la microcuenca del arroyo Videla.

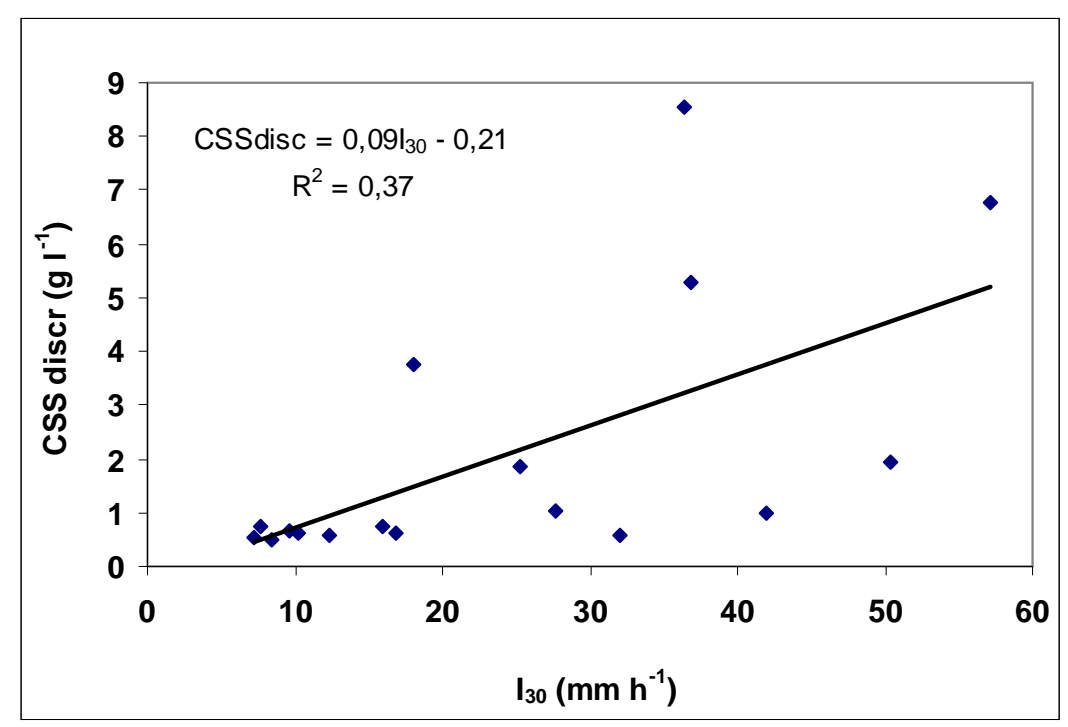

Figura 4.13. Relación entre la concentración de sólidos suspendidos del muestreo discreto y la intensidad máxima de la precipitación en 30 minutos para los 17 eventos estudiados en la microcuenca del arroyo Videla. 


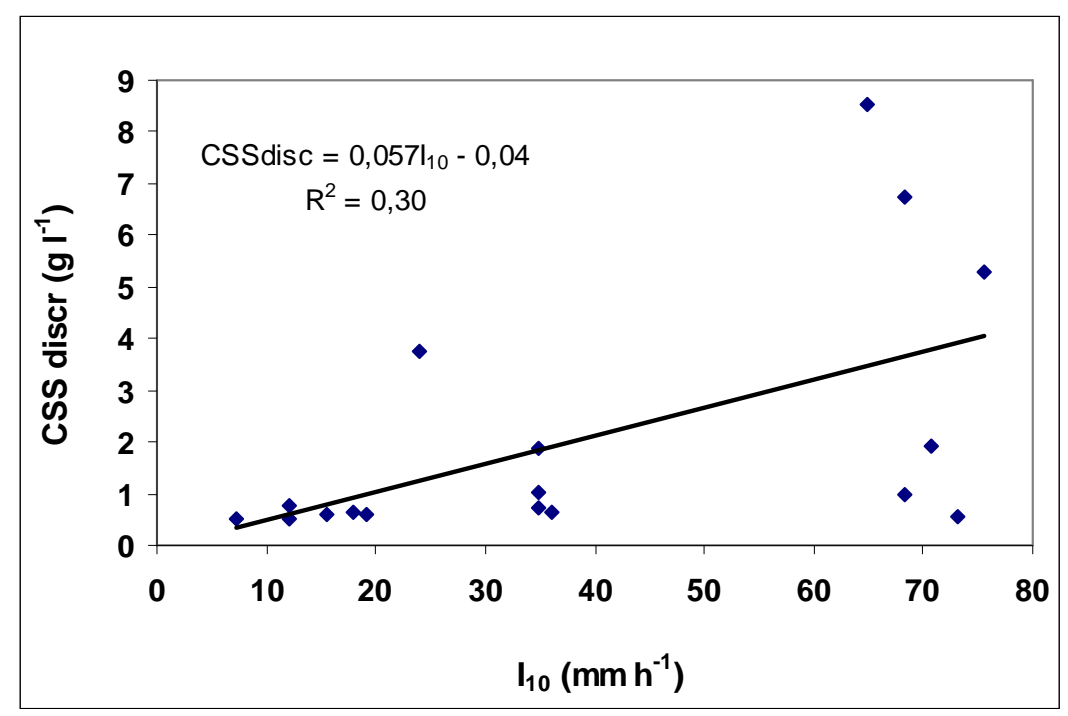

Figura 4.14. Relación entre la concentración de sólidos suspendidos del muestreo discreto y la intensidad máxima de la precipitación en 10 minutos para los 17 eventos estudiados en la microcuenca del arroyo Videla.

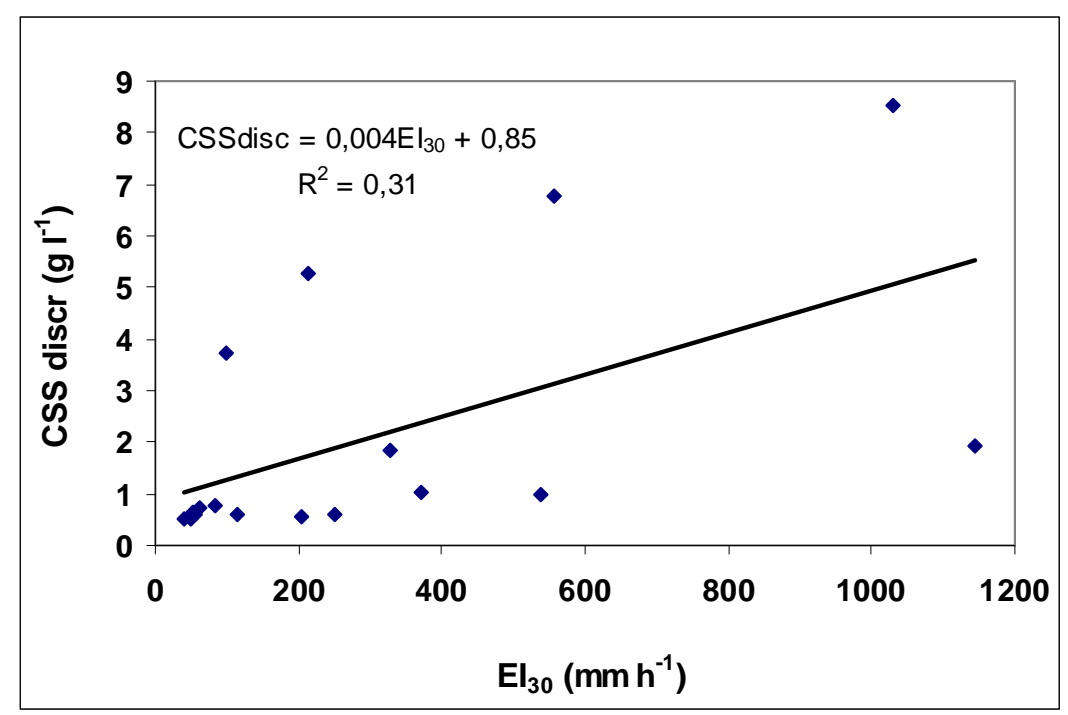

Figura 4.15. Relación entre la concentración de sólidos suspendidos del muestreo discreto y el índice $\mathrm{El}_{30}$ para los 17 eventos estudiados en la microcuenca del arroyo Videla. 


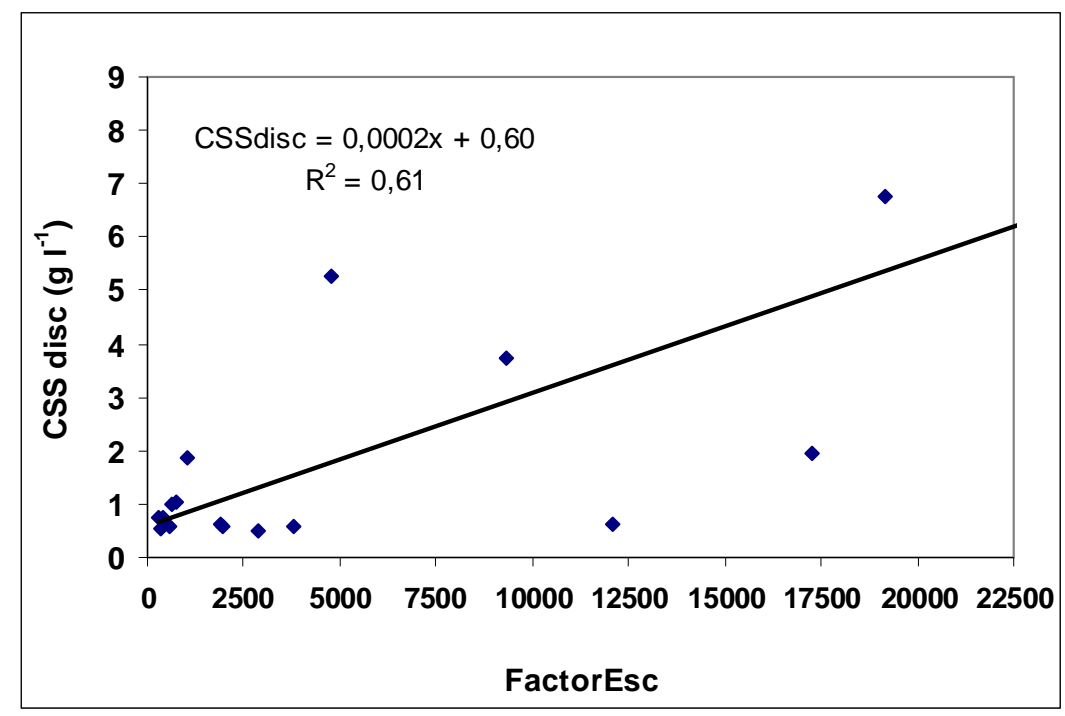

Figura 4.16. Relación entre la concentración de sólidos suspendidos del muestreo discreto y el factor de escurrimiento de MUSLE para los 17 eventos estudiados en la microcuenca del arroyo Videla.

Tabla 4.3. Parámetros de las relaciones de regresión lineal simple entre la concentración del muestreo discreto y el escurrimiento, entre la concentración del muestreo discreto y el caudal base y entre la concentración del muestro discreto y el coeficiente de escurrimiento.

\begin{tabular}{|c|c|c|c|}
\hline Relación & $\begin{array}{c}\text { Ordenada } \\
\text { al origen }\end{array}$ & Pendiente & $\mathrm{R}^{2}$ \\
\hline CSS discreto vs Esc & 0,76 & 0,15 & 0,53 \\
\hline CSS discreto vs Qp & 0,29 & 1,37 & 0,64 \\
\hline CSS discreto vs CE & 0,36 & 0,11 & 0,54 \\
\hline
\end{tabular}

En cuanto a las relaciones entre las variables del escurrimiento y la CSS compuesta, conforme a lo que mostró el análisis de correlación de Pearson, se graficó la relación entre dicha concentración y el CE. De acuerdo con la tendencia que indicaron los datos, se realizó el ajuste a una función exponencial (Figura 4.17), la que presentó un $\mathrm{R}^{2}$ de 0,74 . En este caso, se puede observar que con coeficientes de escurrimiento superiores a $20 \%$ se registrarían incrementos importantes en la concentración compuesta, dado principalmente por 6 eventos que tienen esas características. En igual sentido, las relaciones entre esta CSS y Qp y Qsm mostraron ajuste al mismo tipo de función, pero con dispersión algo mayor, y también con valores de caudal a partir de los cuales esta concentración tendría un aumento considerable, los que fueron cercanos a 1,5 y $0,5 \mathrm{~m}^{3} \mathrm{~s}^{-1}$, respectivamente (Figuras 4.18 y 4.19). La relación entre CSS comp y el CN muestra que con valores de CN superiores a 85 la concentración se incrementaría, pero, en contraste con lo mencionado antes, solo 3 
eventos se ubicarían por encima de tal valor (Figura 4.20). En este caso, el ajuste a la función exponencial presentó un $R^{2}$ menor, que fue de 0,3.

Las tendencias descriptas en este caso indicarían la existencia de valores umbral a partir de los cuales la respuesta a la producción de sedimentos registraría aumentos, lo que podría evidenciarse mejor al considerar las estimaciones de producción total de sedimentos, como será comentado más adelante en este trabajo.

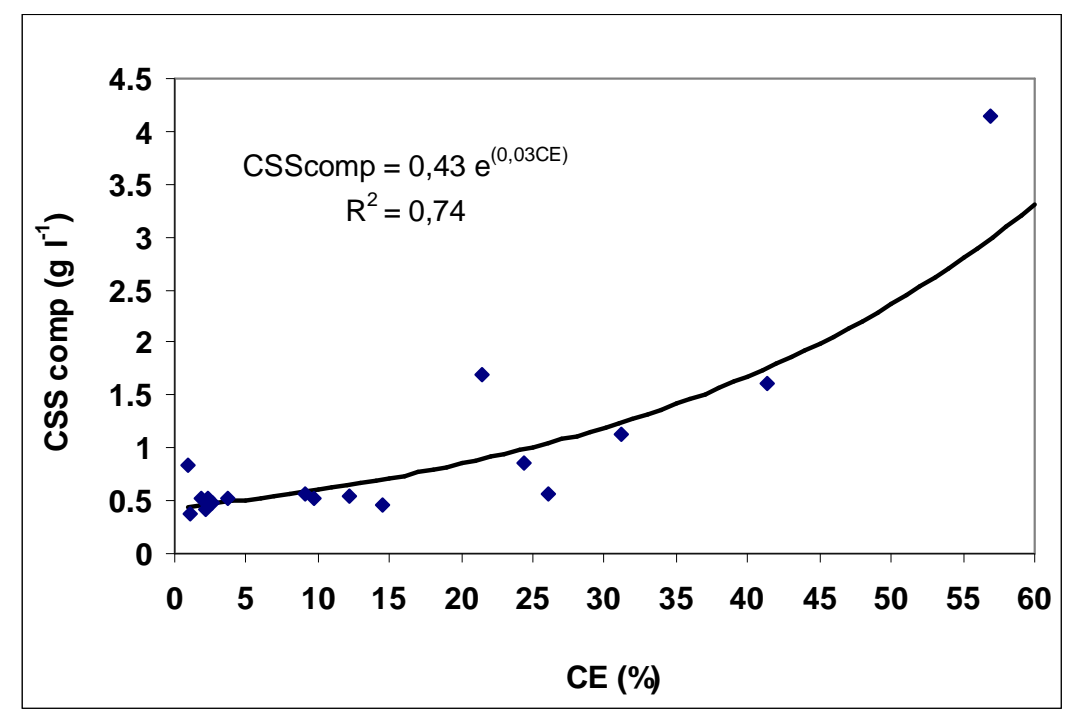

Figura 4.17. Relación entre la concentración de sólidos suspendidos del muestreo compuesto y el coeficiente de escurrimiento.

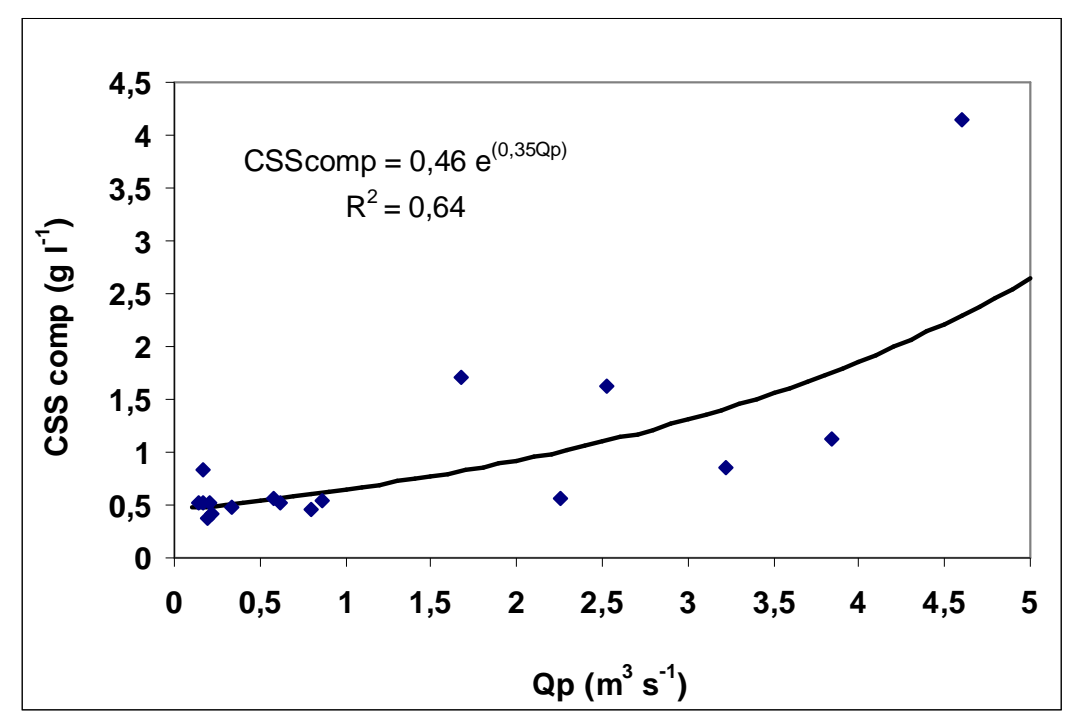

Figura 4.18. Relación entre la concentración de sólidos suspendidos del muestreo compuesto y el caudal pico. 


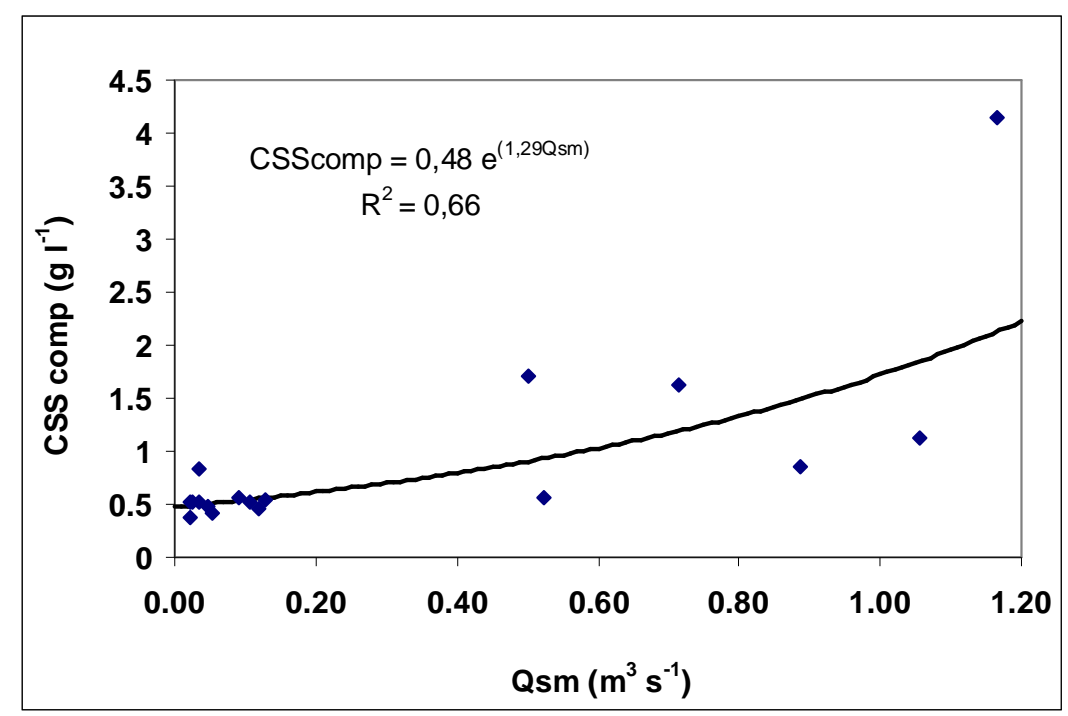

Figura 4.19. Relación entre la concentración de sólidos suspendidos del muestreo compuesto y el caudal superficial medio.

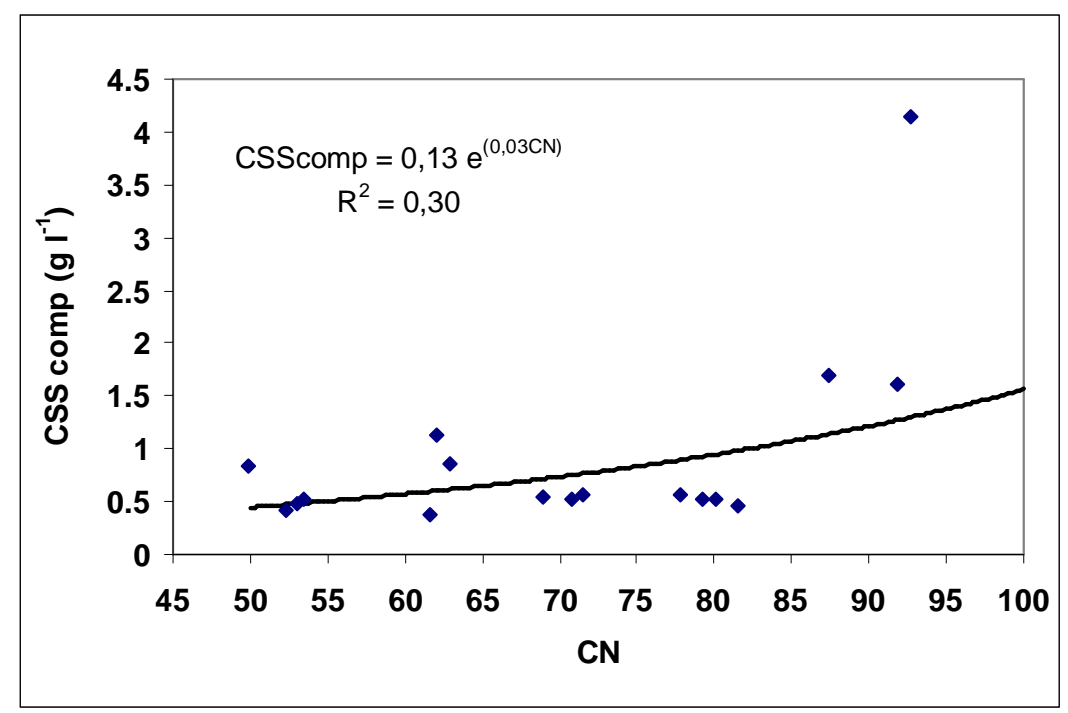

Figura 4.20. Relación entre la concentración de sólidos suspendidos del muestreo compuesto y el CN.

Por otra parte, la importancia que el flujo superficial tuvo en el desprendimiento de material quedó reflejada en la significancia resultante entre la CSS, tanto del muestreo discreto como del compuesto, e $\mathrm{I}_{30}$ (Tabla 3.2) ya que esta última variable considera el incremento en la eficiencia en el desprendimiento asociada al escurrimiento por lluvias que superan la capacidad de infiltración de los suelos (van Dijk et al., 2002). 


\subsection{2.c. Análisis estacional: períodos de baja a media cobertura y de alta cobertura}

Se realizó el análisis de las relaciones entre las variables de la precipitación, el escurrimiento y la concentración de sedimentos del muestreo discreto considerando la estacionalidad en la cobertura vegetal y las precipitaciones. Esto permitió identificar diferencias temporales en los factores involucrados en la respuesta a la producción de sedimentos. Así, durante el período de alta cobertura la CSS del muestreo discreto se correlacionó de manera significativa con la $I_{30}$ y con todas las variables del escurrimiento. Como se discutió en la sección 4.3.1.b., el mecanismo de Horton sería el principal generador del escurrimiento, asociado a la intensidad de las lluvias registradas. De acuerdo a lo que establecen Estrany et al. (2009), en este período la intensidad de la lluvia sería el factor preponderante que movilizaría al flujo y en consecuencia a los sedimentos de las laderas.

Por su parte, en el período de media a baja cobertura, la CSS del muestreo discreto se relacionó de manera significativa únicamente con las variables de la precipitación $\mathrm{Ptot}, \mathrm{El}_{30}$, E y con $I_{30}$, lo que sería indicativo de la respuesta directa entre precipitación y concentración de sedimentos en la cuenca. El análisis de las relaciones entre estas variables permitió el ajuste al modelo exponencial, con valores de $R^{2}$ superiores a 0,8 (Tabla 4.4), lo que indica que la CSS discreta durante el período de baja cobertura no se vincularía de manera lineal con la lluvia y las variables asociadas a ella. A modo de ejemplo, la Figura 4.21 muestra la relación CSS discreta vs $I_{30}$. En coincidencia con lo que se señaló en la sección 4.3.1.b, las relaciones en el periodo de baja cobertura presentaron, en general, menor dispersión en comparación con las respuestas correspondientes al de alta cobertura, como se puede observar al comparar las figuras 4.21 y 4.22 .

Tabla 4.4. Parámetros de las relaciones exponenciales entre la concentración del muestreo discreto correspondiente al período de media a baja cobertura y: la precipitación total, el índice $\mathrm{El}_{30}$, la energía de la precipitación y la intensidad máxima en 30 minutos.

\begin{tabular}{|c|c|c|c|}
\hline Relación & Coeficiente & Exponente & $\mathrm{R}^{2}$ \\
\hline CSS disc vs $\mathrm{P}$ & 0,19 & 0,026 & 0,82 \\
\hline CSS disc vs $\mathrm{EI}_{30}$ & 0,45 & 0,003 & 0,93 \\
\hline CSS comp vs $\mathrm{E}$ & 0,23 & 0,121 & 0,83 \\
\hline CSS comp vs $\mathrm{I}_{30}$ & 0,20 & 0,096 & 0,93 \\
\hline
\end{tabular}




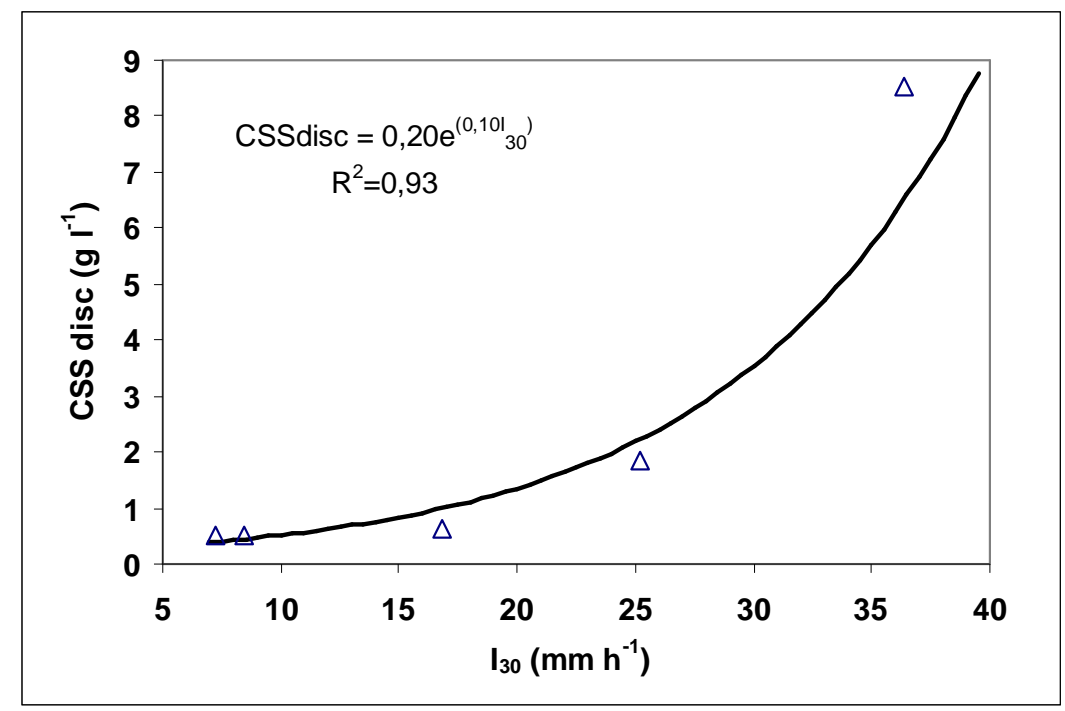

Figura 4.21. Relación entre la concentración de sólidos suspendidos del muestreo discreto y la intensidad máxima de la precipitación en 30 minutos para el período de media a baja cobertura.

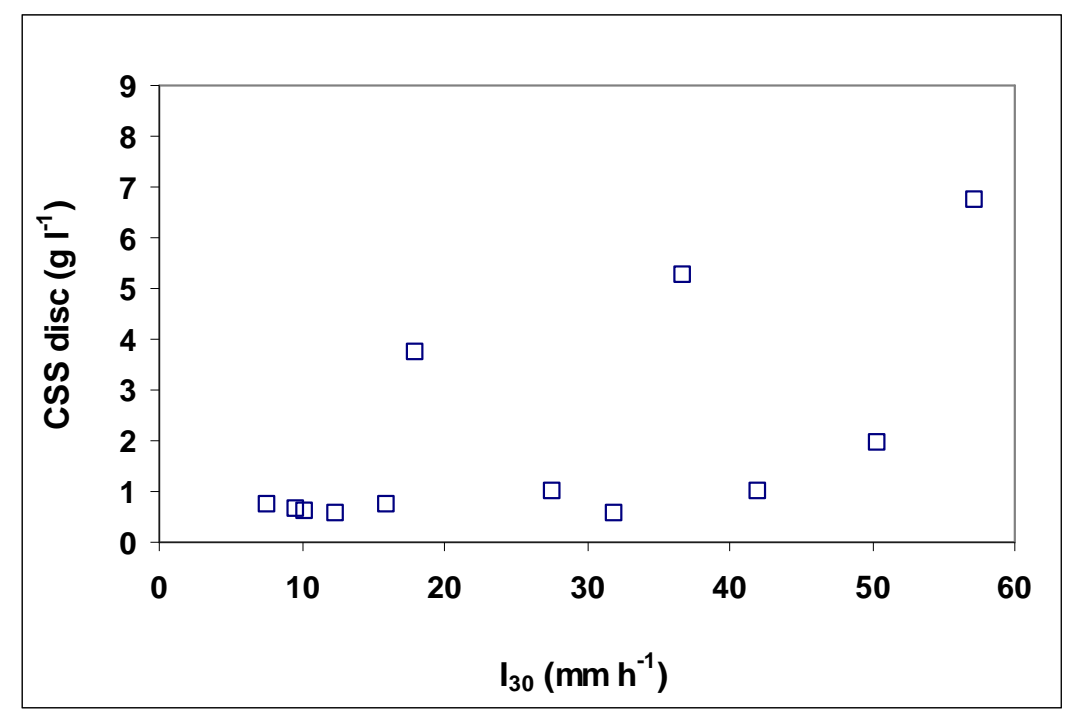

Figura 4.22. Relación entre la concentración de sólidos suspendidos del muestreo discreto y la intensidad máxima de la precipitación en 30 minutos para el período de alta cobertura.

\subsubsection{Relación entre humedad antecedente, escurrimiento y CSS}

La precipitación de los 5 días previos no demostró relaciones significativas con el escurrimiento y sus variables asociadas ni con la concentración de sólidos suspendidos. Esto coincide con lo que mencionan Silveira et al. (2000); Soulis et al. (2009) y Tramblay et al. (2010), en cuanto a que este criterio puede no resultar adecuado para valorar el efecto de 
la humedad previa en la generación de escurrimiento. Por su parte, Nadal-Romero et al. (2008) y Oeurng et al. (2010) coinciden en que la lluvia de los días previos al evento es un factor que no se correlaciona con la producción de sedimentos a escala de cuenca, mientras que Rodríguez-Blanco et al. (2010) y Giménez et al. (2012) sugieren que el caudal base representaría mejor la humedad antecedente del suelo que la lluvia previa. En este sentido, se evaluaron las relaciones tanto entre el escurrimiento y el caudal base $(\mathrm{Qb})$ registrado en la sección de aforos previo a los 17 eventos estudiados como entre la concentración de ambos muestreos realizados y dicho caudal, en términos de regresiones lineales simples. Los resultados, que se presentan en la Tabla 4.5., indican que las regresiones no fueron significativas $(p<0,05)$, por lo cual, para este caso de análisis esta variable tampoco sería representativa de las condiciones previas de las crecidas.

Por otra parte, la reserva de agua en el suelo del día previo a la precipitación sería una variable que permitiría evaluar más directamente la humedad del suelo al involucrar la precipitación, la evapotranspiración potencial y la capacidad de almacenaje del suelo. Si bien, como fuera detallado antes, Ares et al. (2012) reportaron que esta variable había permitido obtener estimaciones del escurrimiento más ajustadas para la cuenca del arroyo Videla, en este caso, considerando el conjunto de los eventos, no demostró relaciones con significancia estadística con el escurrimiento o con la concentración de sedimentos. No obstante, según lo que se detalló en la sección 4.3.1.b. la ResA se relacionó con la generación de escurrimiento superficial de manera exponencial al considerar los eventos del período de media a baja cobertura. Esto se asoció a la posible ocurrencia de flujo subsuperficial y de flujo superficial de saturación. En cuanto a la relación entre esta variable y la CSS, no demostró tendencias claras para el mismo período, tanto para el muestreo discreto como para el compuesto. Dadas estas relaciones, se examinaron también las correspondientes al período de alta cobertura, pero tampoco las tendencias registraron ajuste a alguna función ya sea con el escurrimiento o con la CSS.

De este modo, los resultados obtenidos conducirían a establecer que la producción de sedimentos no estaría relacionada con las condiciones previas de humedad en la microcuenca bajo estudio considerando el conjunto de los eventos individuales. De acuerdo con Zabaleta et al. (2007) y Oeurng et al. (2010) esto indicaría que, en este caso, en la microcuenca se habría producido una respuesta directa a las lluvias en cuanto a producción de sedimentos suspendidos en el período estudiado. Sin embargo, el agrupamiento estacional de los datos permitió hacer una mejor interpretación del efecto de la humedad antecedente en el control del escurrimiento. Así, la reserva de agua en el suelo del día previo a la precipitación evidenciaría la relación entre la humedad antecedente y la 
generación de escurrimiento en el período de media a baja cobertura, en tanto que la respuesta directa a las precipitaciones se daría durante el período de alta cobertura.

Tabla 4.5. Parámetros de las ecuaciones de regresión lineal entre el escurrimiento y el caudal base, entre la concentración de sólidos suspendidos del muestreo discreto y el caudal base y entre la concentración de sólidos suspendidos del muestreo compuesto y el caudal base.

\begin{tabular}{|c|c|c|c|c|}
\hline Relación & $\begin{array}{c}\text { Ordenada } \\
\text { al origen }\end{array}$ & Pendiente & $\mathrm{R}^{2}$ & Valor $\mathrm{p}$ \\
\hline Esc vs Qb & 5,02 & 27,8 & 0,02 & 0,55 \\
\hline CSS disc vs Qb & 0,62 & 10,84 & 0,09 & 0,25 \\
\hline CSS comp vs Qb & 0,07 & 6,32 & 0,21 & 0,06 \\
\hline
\end{tabular}

\subsection{Agrupamiento de los eventos de acuerdo a la interacción lluvia- escorrentía-producción de sedimentos mediante análisis de cluster}

El agrupamiento de los datos obtenido a través del análisis de cluster permitiría identificar diferentes respuestas en cuanto a la producción de sedimentos y los factores relacionados a cada una de ellas. Así, el grupo A se asociaría a la ocurrencia de precipitaciones de baja magnitud e intensidad y por lo tanto de baja erosividad, las cuales habrían provocado un escaso desprendimiento de material edáfico. Mientras tanto, la situación del grupo B presentó una tendencia hacia mayor concentración de sólidos suspendidos, 45,5\% superior a la de los casos del grupo A. Esto podría estar asociado a la erosividad registrada, que en promedio fue de 360,3 MJ mm (ha h) ${ }^{-1}, 81,8 \%$ más elevada que la del grupo A. Los valores descriptos ponen una vez más de manifiesto la no linealidad de los procesos, y en este caso es posible analizar qué factores habrían condicionado dicha respuesta. Se podría postular entonces, asociado al escaso escurrimiento producido en los eventos del grupo B (44\% menor que el correspondiente al grupo A), que el suelo podría haberse desprendido pero no habría encontrado suficiente volumen de agua para ser transportado, limitándose de esta forma la pérdida de suelo (Morgan, 2005). Así, el escaso escurrimiento se podría relacionar con condiciones de humedad previa baja, como fue el caso de los eventos 5 y 6 en particular (Tabla 3.1). A su vez, otro factor podría haber afectado simultáneamente tanto el desprendimiento como el transporte en los eventos 5, 6 y 7 de este grupo ya que dichos eventos se produjeron en los meses de enero y marzo con presencia de cultivos de verano en crecimiento activo, y por consiguiente, con alta cobertura. 
De acuerdo con lo expuesto, podría plantearse que los casos comentados para los grupos $A$ y $B$ en los que el escurrimiento y la CSS fueron bajos, se dieron condiciones favorables para la ocurrencia de erosión de tipo laminar. En este sentido, según lo propuesto por Duvert et al. (2010), se analizó la relación entre la concentración de sedimentos suspendidos y la energía de la precipitación. Esta energía se refiere a la que poseen las gotas de lluvia para desprender y transportar el suelo. Sin embargo, la baja velocidad del flujo puede limitar la capacidad de transporte, lo que resulta en baja producción de sedimentos (van Dijk et al., 2002). En tanto, la intensidad de la lluvia puede influenciar tanto las fases de desprendimiento como la de transporte en este proceso de erosión entre surcos (Gumiere et al., 2009). Las variables se ajustaron a un modelo exponencial, que indica que la energía de las tormentas logró explicar el 53\% de la variabilidad en la concentración de sedimentos suspendidos (Figura 4.23).

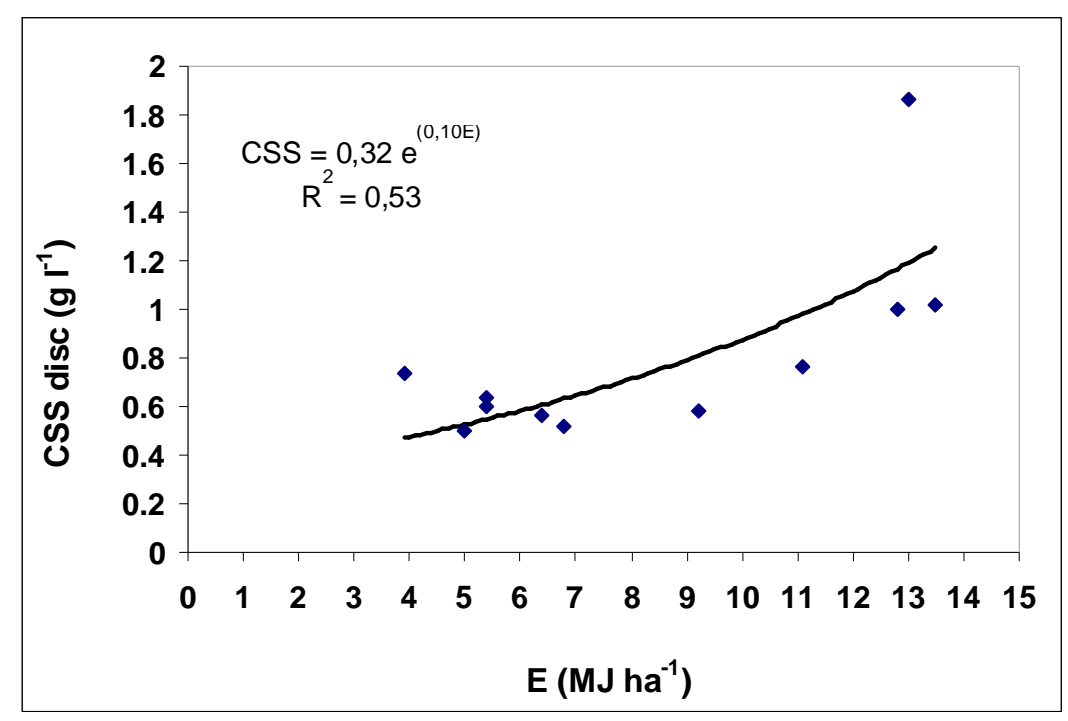

Figura 4.23. Relación entre la concentración de sólidos suspendidos y la energía de la precipitación para los eventos ubicados en los grupos A y B del análisis de cluster $(n=11)$.

Por otra parte, la respuesta representada por los grupos C y D estaba asociada a alta concentración de sedimentos suspendidos. En ellos sería preponderante el rol que cumple el escurrimiento y la proporción de agua que escurre en relación a la lluvia caída (CE), que para estos casos superó el $20 \%$. Si bien para estos casos resultan contrastantes las precipitaciones y las variables asociadas, el escurrimiento habría sido el agente en común que habría provocado el desprendimiento y transporte de suelo. A este factor se habría sumado, de manera importante, el efecto de las lluvias en los eventos ubicados en el grupo C. Distintos aspectos de estos eventos registrados permitirían relacionarlos con erosión en surcos. Duvert et al. (2012) discuten que lluvias de alta intensidad, que pueden estar 
acompañadas de altas magnitudes y energía, generan flujos superficiales altos, que a través de las pendientes escurren y desprenden suelo. Así, el caudal que se produce y su valor máximo, que están en función del flujo superficial, controlan el desprendimiento en dichas pendientes y aportan la energía para el transporte de las partículas. Tal como muestra la Tabla 3.5, los eventos correspondientes a los grupos C y D registraron los mayores Qp y Qsm en promedio que podrían estar relacionados con la alta concentración de material suspendido. En este sentido, se examinó la relación entre la CSS disc y el Qsm (Figura 4.24). Si bien la regresión no fue estadísticamente significativa al nivel $p<0,05$, los datos mostraron una tendencia clara y positiva que indicaría el rol del escurrimiento en estos eventos.

Por otra parte, Morgan (2005) y Cerdan et al. (2002) establecen que la erosión en surcos se caracteriza por generar altas concentraciones de material suspendido, vinculado al flujo de agua concentrado que escurre sobre la superficie, tal como ocurrió en estos eventos. Las condiciones de pendientes del área de estudio, asociadas a presencia de algunos sectores con suelos de profundidad limitada habrían favorecido la generación de dicho flujo en estos casos, al ser superada la capacidad de infiltración ante lluvias importantes.

En relación a la cobertura vegetal, estas crecidas registradas tuvieron lugar bajo los períodos de media a baja cobertura y los de alta cobertura, es decir bajo diversas condiciones que incluyeron predominio de cubierta de rastrojos, cultivos de verano en inicio de crecimiento y otras en pleno desarrollo. Cabe aclarar que, relacionado al sistema de siembra directa implementado en el área de estudio, el suelo estuvo protegido por rastrojos en proporciones que igualaron o superaron el $50 \%$ durante todo el período estudiado. Dicha cobertura contribuye de manera importante a las reducciones en la pérdida de suelo por erosión en surcos en los sistemas bajo siembra directa, debido a la disminución que genera en la erosividad del flujo y su capacidad de transporte (West et al., 1992). Sin embargo, la rugosidad relacionada con la implementación de los cultivos cortando la pendiente como única práctica estructural pudo haber resultado escasa. Edwards et al. (1993) reportaron incrementos en el coeficiente de escurrimiento bajo siembra directa con superficies lisas sin áreas de detención para capturar el agua de lluvia. En este caso, la complejidad de las pendientes en distintos sectores de la microcuenca determinó que en ciertas áreas los cultivos estuvieran orientados en la dirección de la pendiente, lo cual habría favorecido la generación del escurrimiento. Observaciones posteriores a este evento evidenciaron que la masa de agua que escurrió fue tal, que ocasionó el arrastre del rastrojo en áreas de pendiente. Así, con la pérdida de la cobertura la superficie del suelo quedó expuesta a la acción conjunta de la lluvia y del flujo, lo que produjo la formación de surcos y el 
desprendimiento adicional de material. Dos imágenes correspondientes a las condiciones posteriores a la tormenta del evento 9 (17/05/2012) evidencian los surcos formados (Figura 4.25) como ejemplo de lo discutido. En ellas se muestran dos lotes pertenecientes a la microcuenca cubiertos con rastrojo de soja en ese caso, pero también en ese momento podían encontrarse los demás lotes del área de estudio ocupados por residuos de maíz, cebada o trigo.

En esta región, de acuerdo con lo que mencionan Sfeir et al. (1998) el sistema de siembra directa promueve/permite la conservación de las propiedades naturales de sus suelos que, en general poseen texturas franco arcillosas, altos contenidos de materia orgánica, y muy buenas estructuras porosas y estables. Los valores de estabilidad de estructura determinados en una muestra compuesta de los sitios de observaciones de suelos para el cálculo del factor $\mathrm{K}$ son acordes a lo mencionado por los autores citados (Tabla 4.6). Sin embargo, el rol de la cobertura sería fundamental en estos agroecosistemas para atenuar la escorrentía y la pérdida de suelo. En tal sentido, ensayos con simulador de lluvias realizados en sectores representativos de la microcuenca (Ares et al., 2013) permitieron comparar la respuesta ante la lluvia en cuanto al escurrimiento y la pérdida de suelo en microparcelas cubiertas con rastrojo y en otras descubiertas (Tabla 4.7). En dicha oportunidad se trabajó con intensidades de lluvia de $120 \mathrm{~mm} \mathrm{~h}^{-1}$ y volúmenes totales de 90 a $240 \mathrm{~mm}$ habida cuenta que al emplear intensidades menores, no se lograba generar escurrimientos medibles durante la primera hora de ensayo. Si bien se advierte que existieron diferencias en las condiciones iniciales de humedad edáfica entre simulaciones, las mismas habrían sido poco importantes frente a los grandes volúmenes de agua aplicados. Así, en suelo cubierto el coeficiente de escurrimiento promedio fue de 3,7\%, y la pérdida de suelo de 3,1 $\mathrm{mg}$ de sedimento por $\mathrm{mm}$ de precipitación aplicado. Mientras tanto, las parcelas descubiertas bajo igual intensidad alcanzaron un coeficiente de escurrimiento de $28,8 \%$, con una pérdida de suelo de $64,2 \mathrm{mg}$ de sedimento por $\mathrm{mm}$ de precipitación aplicado. Estos resultados coinciden con lo que señalan Böhm \& Gerold (1995) y Engel et al. (2009) quienes encontraron que la cubierta vegetal es un factor primordial para reducir el escurrimiento y la pérdida de suelo.

Durante el período analizado, en este sistema bajo siembra directa la mayoría de los eventos producidos estuvieron relacionados con menores escurrimientos y concentración de sedimentos. Mientras tanto, una menor proporción de eventos habrían generado alto escurrimiento y alta concentración de sólidos suspendidos. Estos resultados coinciden con lo que establecen Shipitalo \& Edwards (1998) y Estrany et al. (2009), en cuanto a que la mayor parte de la pérdida de suelo se produce en eventos menos frecuentes. La erosión 
laminar sería el mecanismo de erosión predominante en los primeros casos y en los más frecuentes, y la erosión en surcos la asociada al segundo grupo de eventos. En este agroecosistema, si bien el manejo bajo siembra directa permite mantener las propiedades favorables de los suelos, su implementación en áreas de pendiente sin estructuras de conservación del suelo proporcionaría escasa rugosidad ante estos eventos de menor frecuencia y conduciría a, través del escurrimiento, a la pérdida de la cobertura superficial y por lo tanto de su efecto beneficioso en la preservación del agua y del suelo.

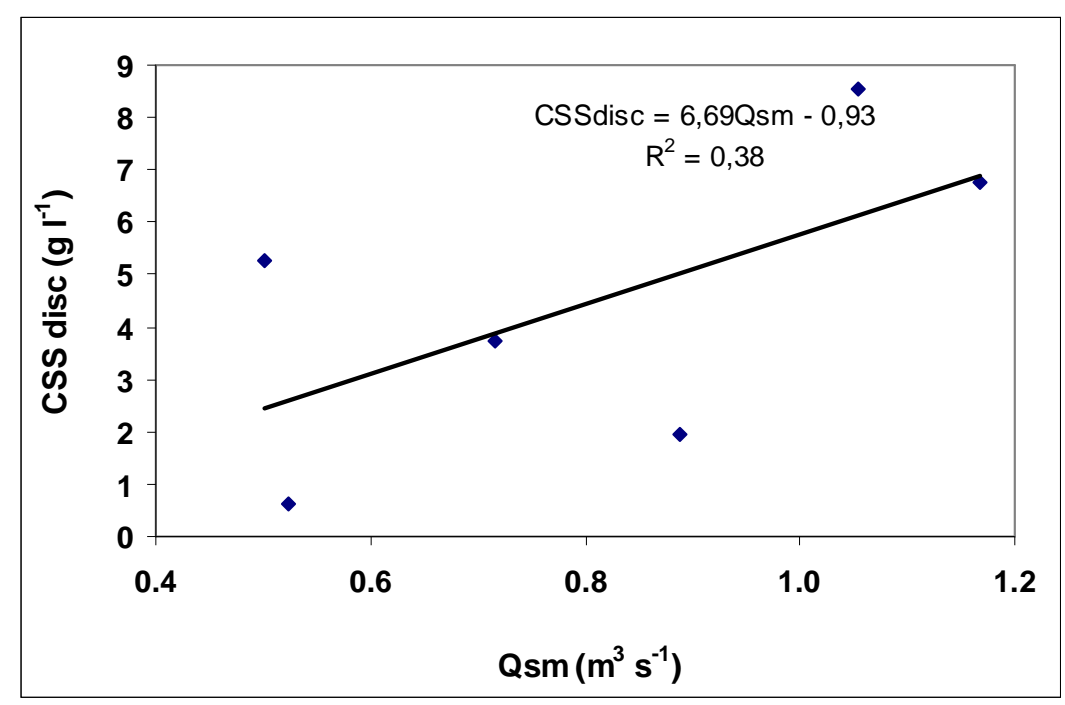

Figura 4.24. Relación entre la concentración de sólidos suspendidos y el caudal superficial medio de los eventos ubicados en los grupos $C$ y $D$ del análisis de cluster $(n=6)$.
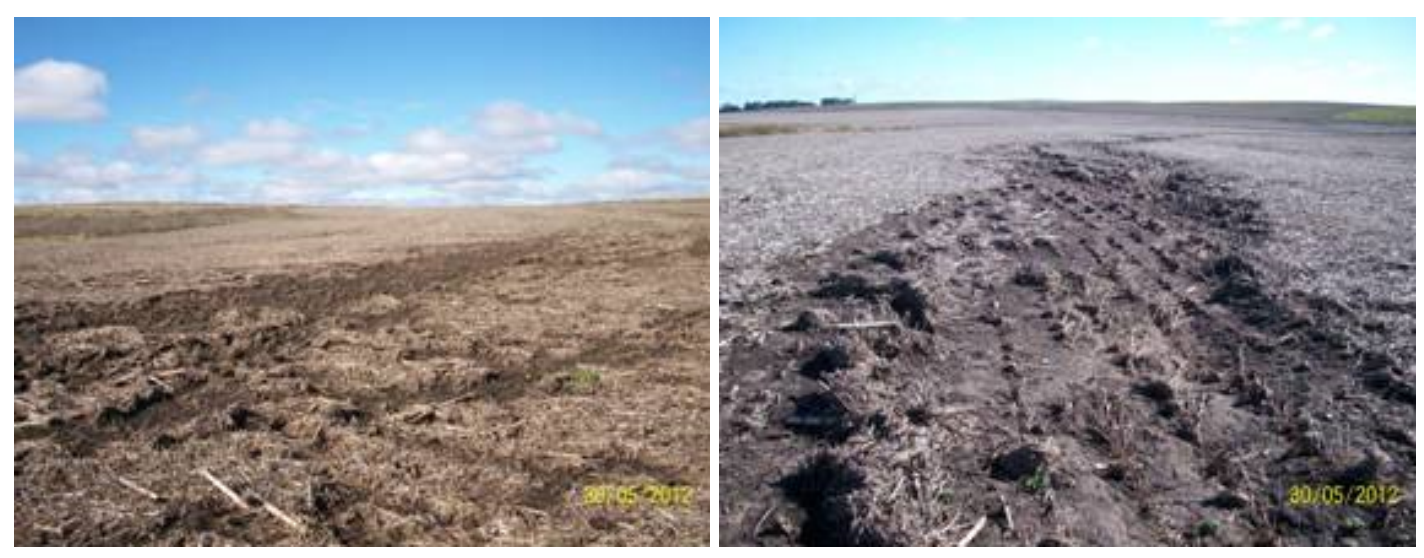

Figura 4.25. Fotografías que muestran surcos formados en el área de estudio durante la tormenta del día 17/05/2012. 
Tabla 4.6. Estabilidad estructural por el método de Le Bissonnais (1996) e hidrofobicidad por el método citado por Chenu et al. (2000), correspondientes a una muestra compuesta de los sitios de observaciones de suelos para el cálculo del factor $\mathrm{K}$.

Referencias: (1) pretratamiento de humectación rápida por inmersión, (2) pretratamiento de humectación lenta por capilaridad, (3) pretratamiento por agitación mecánica luego de la prehumectación, (4) valor de la mediana de hidrofobicidad, (5) valor de la media de hidrofobicidad. (Comunicación personal de Filipe B. Kraemer y Patricia Fernández).

\begin{tabular}{|c|c|c|c|c|}
\hline \multicolumn{3}{|c|}{ Estabilidad estructural } & \multicolumn{2}{c|}{ Hidrofobicidad } \\
Diámetro medio ponderado $(\mathrm{mm})$ & $(\mathrm{s})$ \\
\hline$(1)$ & $(2)$ & $(3)$ & $(4)$ & $(5)$ \\
\hline 1,79 & 3,10 & 3,30 & 3,55 & 5,08 \\
\hline
\end{tabular}

Tabla 4.7. Resultados de ensayos de simulación de lluvia realizados en el área de estudio. Referencias: TIF: tasa de infiltración final obtenida en cada ensayo ( $\mathrm{mm} \mathrm{h}$-1). (Adaptado de Ares et al., 2013)

\begin{tabular}{|c|c|c|c|c|c|c|}
\hline $\begin{array}{c}\text { Condición } \\
\text { superficial del } \\
\text { suelo }\end{array}$ & $\begin{array}{c}\text { Humedad } \\
\text { gravimétrica } \\
\text { inicial } \\
(0-30 \mathrm{~cm}) \\
(\%)\end{array}$ & $\begin{array}{c}\mathrm{P} \\
\text { aplicada } \\
(\mathrm{mm})\end{array}$ & $\begin{array}{c}\text { Esc total } \\
(\mathrm{mm})\end{array}$ & $\begin{array}{c}\text { CE } \\
(\%)\end{array}$ & $\begin{array}{c}\text { TIF } \\
\left(\mathrm{mm} \mathrm{h}^{-1}\right)\end{array}$ & $\begin{array}{c}\text { Sedimentos } \\
\text { producidos } \\
\left(\mathrm{mg} \mathrm{mm} \mathrm{lluvia}^{-1}\right)\end{array}$ \\
\hline Cubierto & 19,6 & 180 & 8,3 & 4,6 & 116,0 & 2,3 \\
\hline Cubierto & 25,6 & 240 & 6,7 & 2,8 & 115,0 & 3,9 \\
\hline Desnudo & 27,9 & 120 & 38,0 & 31,7 & 42,6 & 93,8 \\
\hline Desnudo & 28,0 & 135 & 35,0 & 25,9 & 27,1 & 34,5 \\
\hline
\end{tabular}

\subsection{Producción de sedimentos estimada}

Se estimaron valores de producción de sedimentos. Estos valores pueden ser considerados únicamente como orientativos, ya que dicho muestreo tuvo lugar durante $1,5 \mathrm{~h}$ durante la crecida, como fuera mencionado antes. De tal modo, ese período de recolección correspondió a distintas proporciones de cada evento, según la duración total de éstos. En la Tabla 4.8 se presentan esas proporciones, expresadas como valor porcentual de la crecida, en la que se puede observar que el muestreo se extendió entre el $2 \%$ y el $11 \%$ de la duración total del evento. 
Tabla 4.8. Duración del muestreo compuesto en relación al tiempo total de la crecida expresada como valor porcentual, correspondiente a los 17 eventos analizados en el área de estudio.

\begin{tabular}{|c|c|c|}
\hline № evento & Fecha & $\begin{array}{c}\text { Proporción de la crecida } \\
\text { correspondiente al } \\
\text { muestreo compuesto (\%) }\end{array}$ \\
\hline 1 & $15 / 01 / 2011$ & 3,5 \\
\hline 2 & $01 / 05 / 2011$ & 2,9 \\
\hline 3 & $19 / 07 / 2011$ & 5,6 \\
\hline 4 & $08 / 11 / 2011$ & 6,1 \\
\hline 5 & $10 / 01 / 2012$ & 3,1 \\
\hline 6 & $05 / 03 / 2012$ & 3,8 \\
\hline 7 & $11 / 03 / 2012$ & 2,1 \\
\hline 8 & $18 / 04 / 2012$ & 5,9 \\
\hline 9 & $17 / 05 / 2012$ & 2,5 \\
\hline 10 & $23 / 08 / 2012$ & 2,7 \\
\hline 11 & $03 / 09 / 2012$ & 2,9 \\
\hline 12 & $05 / 10 / 2012$ & 3,4 \\
\hline 13 & $15 / 10 / 2012$ & 2,0 \\
\hline 14 & $22 / 11 / 2012$ & 3,9 \\
\hline 15 & $05 / 12 / 2012$ & 6,7 \\
\hline 16 & $19 / 12 / 2012$ & 5,5 \\
\hline 17 & $28 / 12 / 2012$ & 10,7 \\
\hline & & \\
\hline
\end{tabular}

Se considera que, en su mayoría, los mismos corresponderían a valores de concentración superiores a los que posiblemente hayan sido generados en los eventos en promedio. En 16 de los 17 casos estudiados esto estaría explicado por la diferencia entre las concentraciones que fueron medidas en el muestreo discreto y el compuesto. Como ya fue comentado, el muestreo discreto es aquel que se produjo en primer lugar y durante 7 minutos, y registró mayor concentración que el compuesto, que se extendió en los 90 minutos posteriores al inicio del discreto. Esto indicaría entonces que la concentración desde el inicio de la recolección tendería a disminuir, por lo cual las estimaciones realizadas probablemente superarían a las realmente producidas en los eventos. Por otra parte, solamente en uno de los casos (evento №4, de fecha 08/11/2011) la concentración del muestreo compuesto fue mayor que la del discreto. Así, para esta crecida el valor calculado estaría por debajo del que posiblemente se haya registrado. 
De acuerdo con las limitaciones que poseen estos cálculos y según lo que establece Walling (1994) estas mediciones de producción de sedimentos no pueden ser utilizadas para expresar niveles de erosión real. Aún si se contara con el valor observado de cada crecida, dicho autor señala que la producción de sedimentos da información de los niveles promedio de erosión. Ello estaría asociado a que solamente una pequeña proporción del sedimento desprendido en una cuenca alcanza su salida, debido a la depositación, al almacenamiento temporal en los pies de las pendientes, en el plano de inundación del curso de agua, o asimismo en el canal. La magnitud de estas pérdidas tiende a incrementarse con el tamaño de las cuencas consideradas. Otro aspecto que debe tenerse en cuenta en este caso es la discontinuidad temporal involucrada en la producción de sedimentos. Así, el material desprendido en un sitio puede ser almacenado transitoriamente en otro y ser removilizado más tarde, e incluso varias veces antes de alcanzar la salida de la cuenca.

\subsubsection{Relaciones entre la producción de sedimentos estimada y las variables estudiadas}

\subsection{1.a. Análisis a escala de eventos individuales}

Como se describió en la sección 3.5.1.a., la producción de sedimentos estimada se relacionó de manera significativa con $\mathrm{I}_{30}$ y con el índice $\mathrm{El}_{30}$. Las Figuras 4.26 y 4.27 muestran tales relaciones, con la ecuación de ajuste del modelo lineal. En ellas se observa, de acuerdo con los ajustes que la dispersión de los datos es menor con la intensidad máxima de la lluvia en 30 minutos.

También se examinaron las relaciones entre la producción estimada de sedimentos y las variables del escurrimiento. En la Figura 4.28 se presenta la correspondiente al caudal pico. Si bien los datos se ajustan muy bien a una función exponencial $\left(R^{2}=0,91\right)$, con el objeto de facilitar el análisis, se ajustaron 3 líneas de tendencia, las que se presentan en la Figura 4.29., y que permitirían discutir algunos aspectos. Tales tendencias diferenciarían valores de caudal pico a partir de los cuales se considera que se producirían cambios en la respuesta en cuanto a la producción de sedimentos. Dichos valores límite serían cercanos a $1 \mathrm{~m}^{3} \mathrm{~s}^{-1}$ y a $3,25 \mathrm{~m}^{3} \mathrm{~s}^{-1}$. Con respecto a las 3 ecuaciones de ajuste, demostraron significancia estadística $(p<0,05)$, y sus pendientes evidenciarían esos cambios en la respuesta al caudal pico (Tabla 4.). Para el primer tramo de ajuste la pendiente fue de 17,7 , en tanto que para el segundo fue de 44,5 , es decir que cada metro cúbico de aumento en el caudal pico correspondería a 
una producción estimada de sedimentos 2,5 veces mayor con respecto a la obtenida en el tramo inicial. Pero las diferencias serían aún más importantes para el último tercio de ajuste, en el cual la pendiente fue de 263,8 , es decir que la producción de sedimentos se incrementaría $83 \%$ con caudales pico superiores a $3,25 \mathrm{~m}^{3} \mathrm{~s}^{-1}$ para cada unidad de aumento de caudal pico.

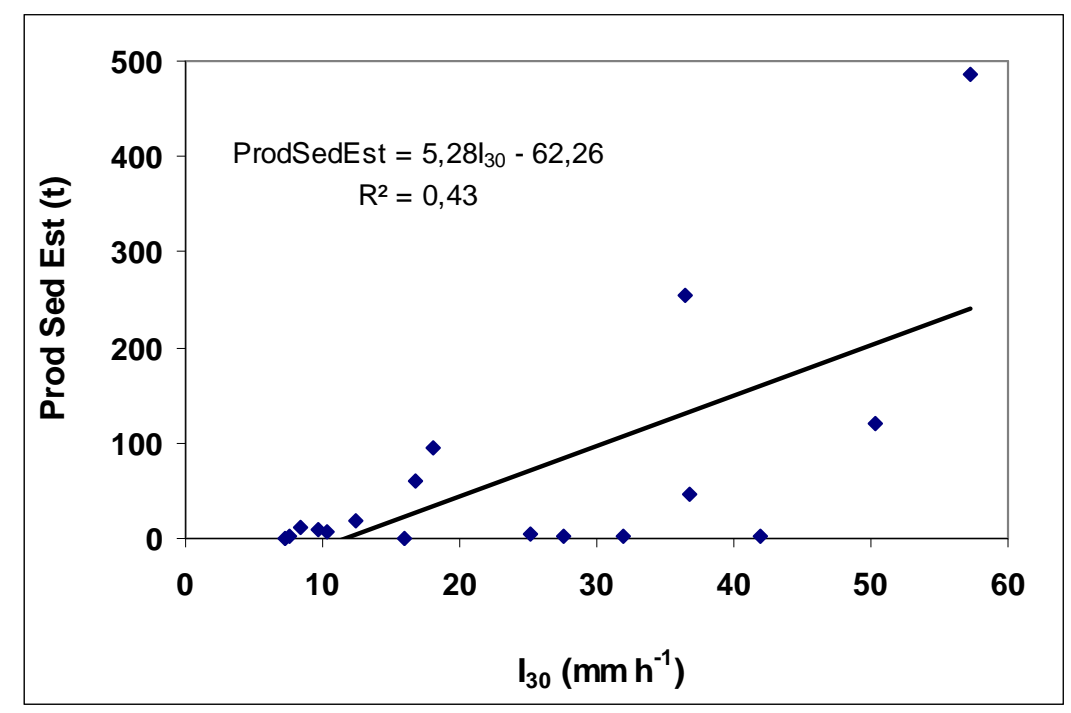

Figura 4.26. Relación entre la producción de sedimentos estimada y la intensidad máxima de la precipitación en 30 minutos para los 17 eventos registrados en la microcuenca del arroyo Videla.

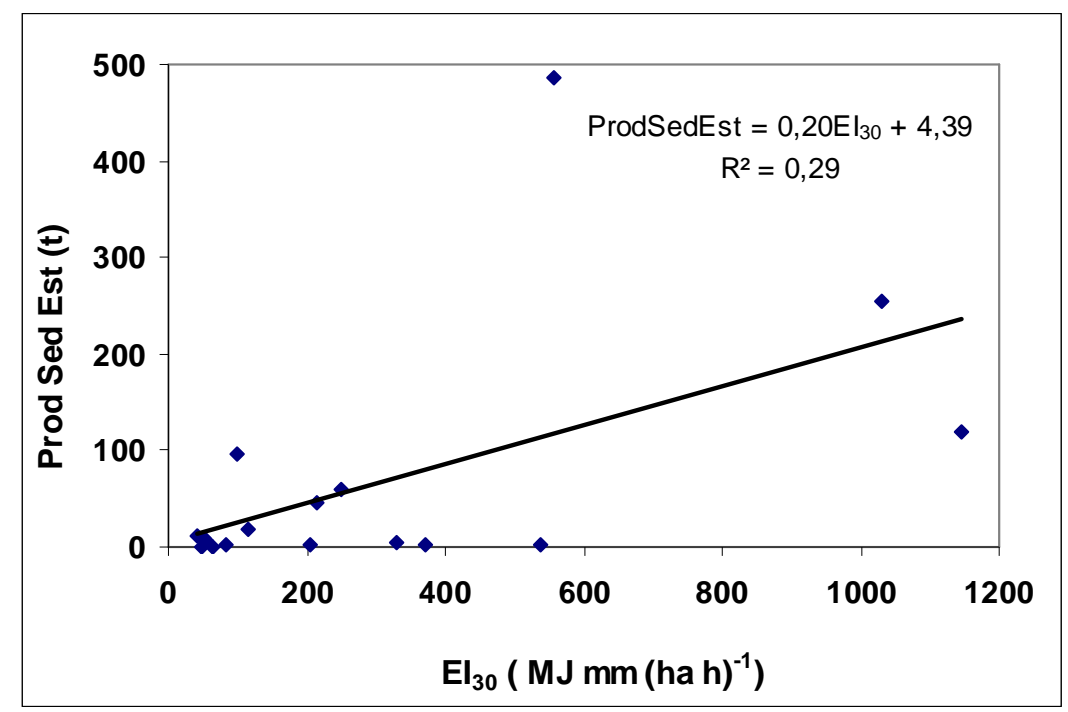

Figura 4.27. Relación entre la producción de sedimentos estimada y el índice $\mathrm{El}_{30}$ para los 17 eventos registrados en la microcuenca del arroyo Videla. 


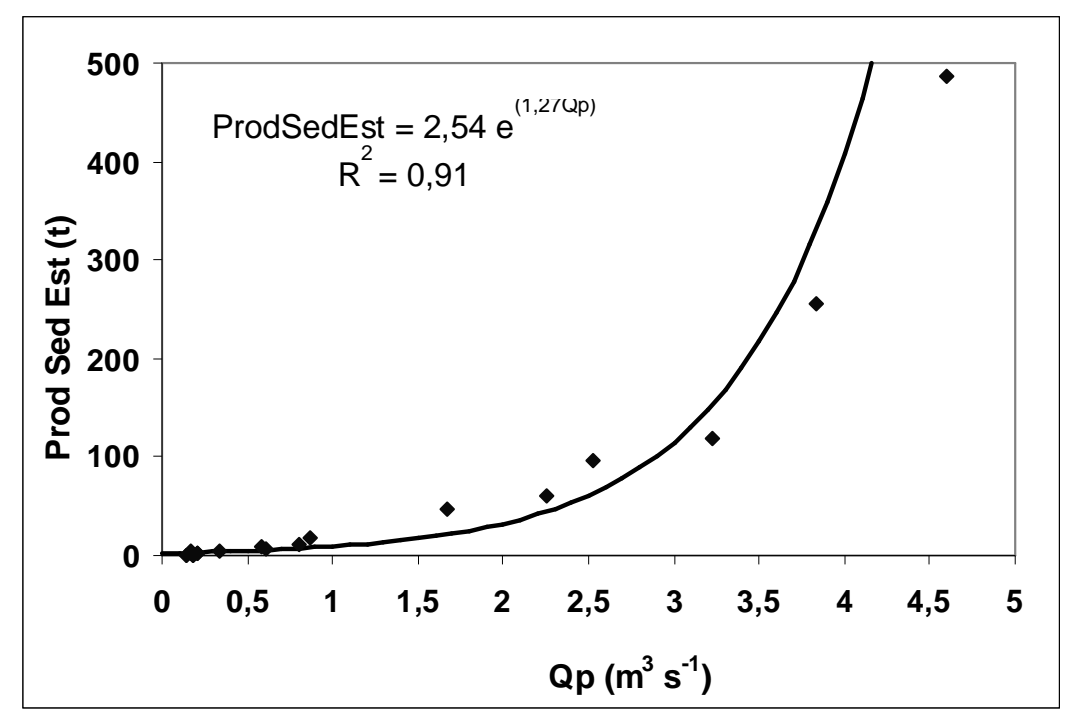

Figura 4.28. Relación entre la producción de sedimentos estimada y el caudal pico para los 17 eventos registrados en la microcuenca del arroyo Videla.

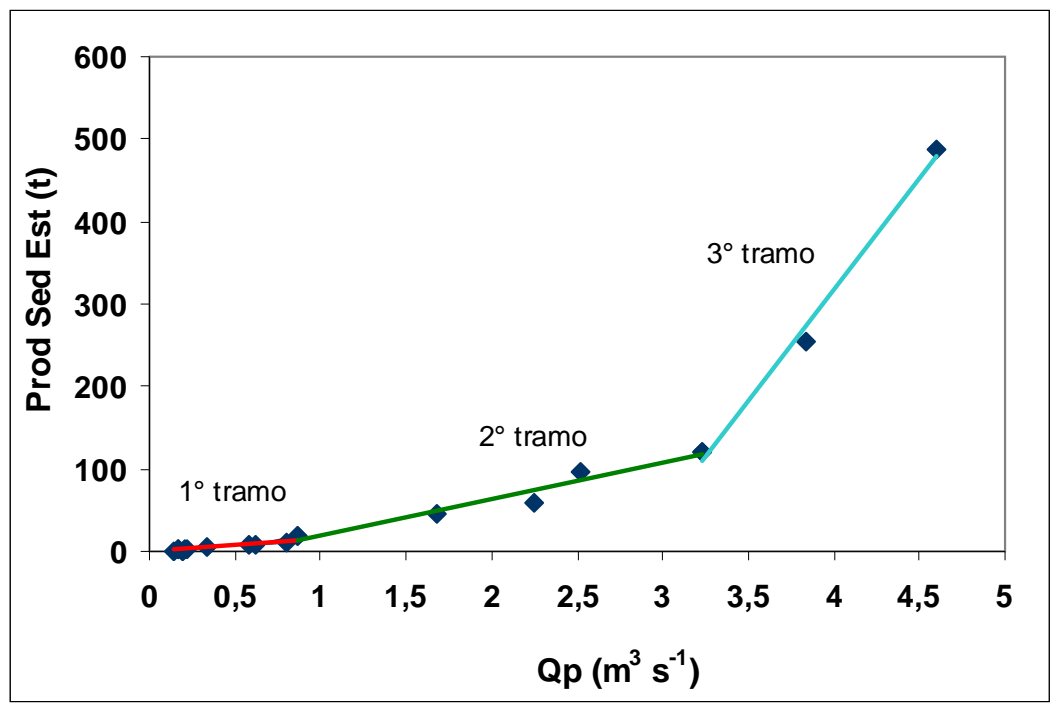

Figura 4.29. Relación entre la producción de sedimentos estimada y el caudal pico para los

17 eventos registrados en la microcuenca del arroyo Videla. Líneas de ajuste correspondientes a los tres tramos identificados en la relación entre las variables.

Tabla 4.9. Parámetros de las ecuaciones de ajuste lineal por tramos entre la producción de sedimentos estimada y el caudal pico.

\begin{tabular}{|c|c|c|c|c|}
\hline & $\begin{array}{c}\text { Ordenada al } \\
\text { origen }\end{array}$ & Pendiente & $\mathrm{R}^{2}$ & Valor $\mathrm{p}$ \\
\hline $1^{\circ}$ tramo & $-1,1$ & 17,7 & 0,88 & $<0,0001$ \\
\hline $2^{\circ}$ tramo & $-26,3$ & 44,5 & 0,93 & 0,0074 \\
\hline $3^{\circ}$ tramo & $-733,05$ & 263,8 & 0,99 & 0,0429 \\
\hline
\end{tabular}


En relación a esto, en la sección 4.3.2.b se identificaron valores de las variables del escurrimiento a partir de los cuales se observaban cambios en la concentración de sedimentos del muestreo compuesto. Las crecidas que se ubicaban a partir de esos valores eran las correspondientes al segundo y tercer tramo de ajuste antes mencionado. Asimismo, esos eventos son aquellos que fueron asociados en el análisis de cluster en los grupos $\mathrm{C}$ y $D$, los cuales se relacionaron con la erosión en surcos. De este modo, el análisis realizado en la presente sección encuentra vinculación con otros comentados anteriormente. Si bien se basa en estimaciones de producción de sedimentos, permitiría identificar valores a partir de los cuales posiblemente se habrían dado cambios en la producción de sedimentos en relación a los mecanismos de erosión predominantes en la microcuenca durante el período de estudio. Esto está vinculado a lo que menciona Cammeraat (2004), al referirse al concepto de valores umbral, que puede ser utilizado para establecer diferencias en cuanto a la dominancia de distintos procesos. Por ello, con valores de caudal pico inferiores a $1 \mathrm{~m}^{3} \mathrm{~s}^{-1}$ habría predominancia de erosión laminar, con un ajuste de la tendencia con un $\mathrm{R}^{2}$ algo menor probablemente asociado a la menor importancia que posee esta variable en este tipo de erosión. En tanto, al superar tal caudal pico, se daría erosión en surcos, con un proceso más intenso con caudales mayores que $3,25 \mathrm{~m}^{3} \mathrm{~s}^{-1}$, que probablemente implique cambios importantes en el sistema. De acuerdo con Duvert et al. (2012), tanto el máximo flujo como su energía son función del caudal pico. Por lo tanto esas variables controlarían el transporte de sedimentos y la capacidad de arrastre del cauce. Así, las condiciones del flujo con respecto a su masa y a su alta velocidad en las laderas podrían estar asociadas a los cambios mencionados en la microcuenca. Dadas estas condiciones se habría expresado el alto poder erosivo que posee el escurrimiento concentrado (Morgan, 2005). Además, es posible que las vías formadas por los surcos se hayan activado como caminos preferenciales para la circulación del agua, y que también que en estos eventos se haya incrementado la conectividad en el paisaje. Autores como de Vente et al. (2007) vinculan el aumento en la producción de material erosionado en la salida de las cuencas a tal incremento en la conectividad, lo que sucede cuando se producen cambios de erosión de tipo laminar a erosión en surcos, de esta a erosión en cárcavas efímeras, hasta la formación de cárcavas más profundas.

Por su parte, y de acuerdo con Ritter et al. (1999), los valores umbrales pueden ser indicativos de cambios en las condiciones del equilibrio de los sistemas naturales. Así, podría considerarse que haya sucedido una modificación en la resiliencia del sistema. Es decir, que hasta cierto valor, en este caso de caudal pico, la cuenca con las características y propiedades de sus suelos de alto contenido de materia orgánica, estabilidad de su 
estructura, que le confieren alta capacidad de infiltración, tal como mostraron ensayos de simulación de lluvia realizados en el área de estudio (Ares et al., 2013), podría expresar una capacidad de regulación ante eventos de determinadas características. Luego, superado ese nivel en cuanto a los factores relacionados al flujo y su energía antes detallados, el sistema no podría manifestar dicha capacidad de regulación, lo que implicaría ese cambio en las condiciones de equilibrio.

Por otra parte, se encontró una tendencia comparable a la descripta anteriormente en cuanto a la relación entre la producción estimada de sedimentos y el caudal superficial medio. En este caso también el ajuste exponencial resultó adecuado (Figura 4.30), pueden identificarse cambios importantes en la respuesta en cuanto a la producción de sedimentos estimada en función de esta variable. Por ejemplo, para un Qsm de 0,6 $\mathrm{m}^{3} \mathrm{~s}^{-1}$ la producción estimada de sedimentos sería de $47 \mathrm{t}$, mientras que para otro de $0,8 \mathrm{~m}^{3} \mathrm{~s}^{-1}$ tal valor sería de $115 \mathrm{t}$, y con $1 \mathrm{~m}^{3} \mathrm{~s}^{-1}$ de Qsm, el sedimento producido sería de $284 \mathrm{t}$. Es decir, que entre 0,8 y $1 \mathrm{~m}^{3} \mathrm{~s}^{-1}$ se podría encontrar el cambio en la respuesta.

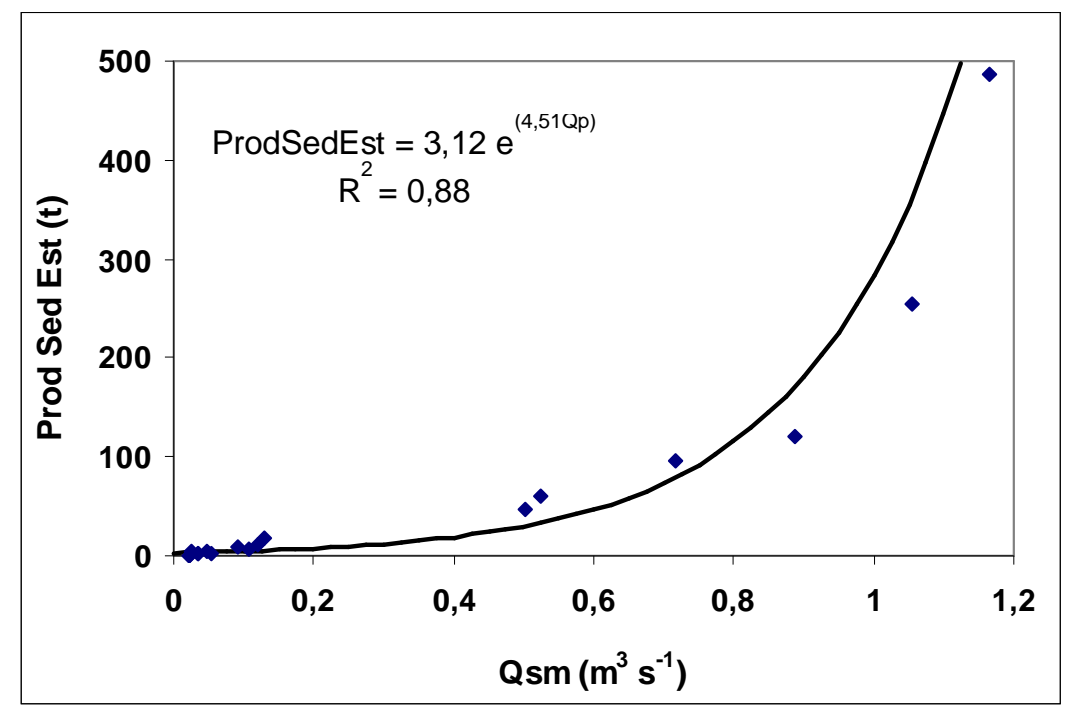

Figura 4.30. Relación entre la producción de sedimentos estimada y el caudal superficial medio para los 17 eventos registrados en la microcuenca del arroyo Videla.

Las relaciones con el CE y el factor de escurrimiento de MUSLE también presentaron ajustes satisfactorios a un modelo exponencial (Figuras 4.31 y 4.32), pero con una dispersión algo mayor, lo que no permitiría definir predicciones con la calidad de ajuste de los casos anteriores, sobre todo para los valores más altos de sedimentos estimados. 
Por último, de acuerdo con lo analizado antes, las relaciones entre la producción de sedimentos estimada y las variables asociadas al escurrimiento presentaron mejores ajustes que las correspondientes a las variables de la precipitación. Si bien se trata de relaciones con valores orientativos de la producción de sedimentos, se puede mencionar que estos resultados coinciden con los obtenidos por otros autores en estudios con valores observados de la producción total de sedimentos (Mathys et al., 2003; Soler et al., 2007).

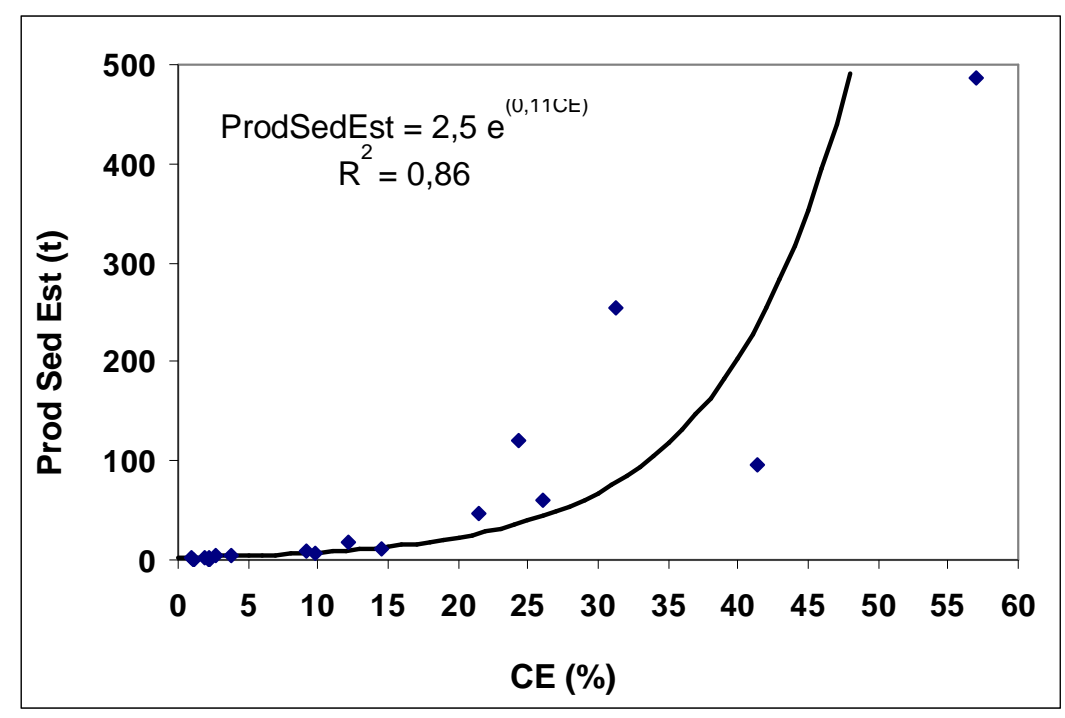

Figura 4.31. Relación entre la producción de sedimentos estimada y el coeficiente de escurrimiento para los 17 eventos registrados en la microcuenca del arroyo Videla.

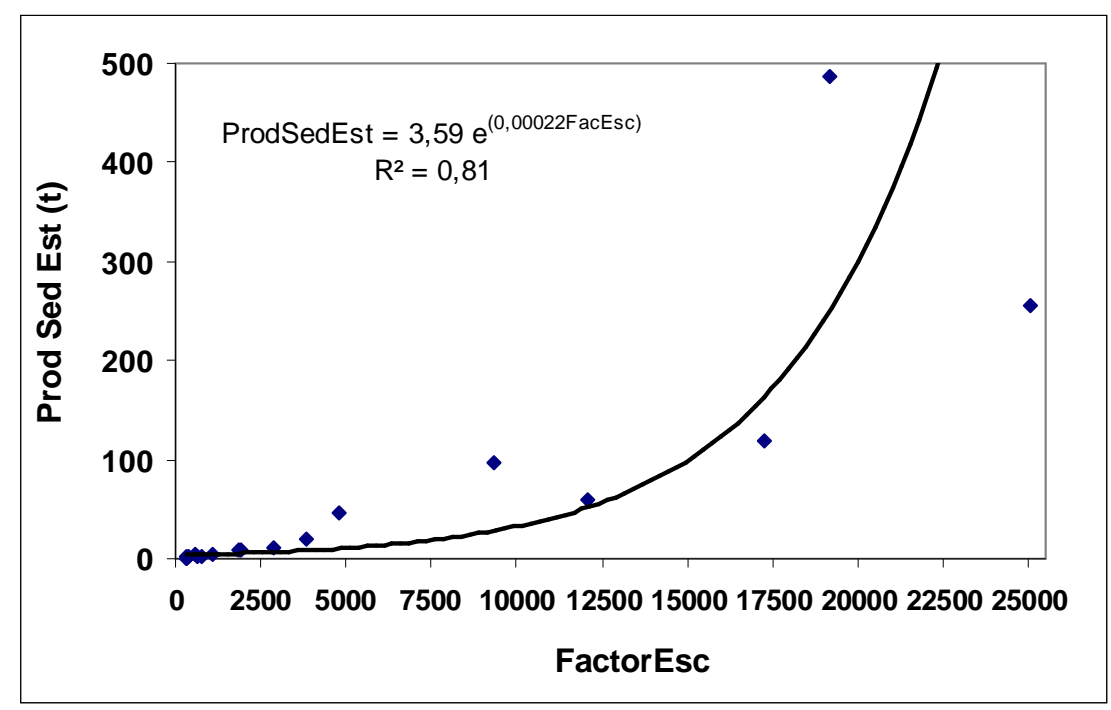

Figura 4.32. Relación entre la producción de sedimentos estimada y el factor de escurrimiento del modelo MUSLE para los 17 eventos registrados en la microcuenca del arroyo Videla. 


\subsection{1.b. Análisis estacional: períodos de baja a media cobertura y de alta cobertura}

El análisis de las relaciones entre la producción de sedimentos estimada y las variables de la precipitación y el escurrimiento mostró diferentes asociaciones al considerar períodos de alta y de media a baja cobertura. Con respecto al período de alta cobertura, dichas relaciones coinciden con las que se obtuvieron entre la CSS disc y las variables mencionadas y que fueron discutidas en la sección 4.3.2.c. Así, nuevamente se podría vincular a la $I_{30}$ con su influencia en la generación de escurrimiento y a la acción combinada de estos factores en la producción de sedimentos estimada.

Por su parte, en el período de media a baja cobertura, además de las relaciones entre la producción de sedimentos estimada y las variables de la precipitación, el Qp, el Qsm y el factor de escurrimiento se vincularían con dicha producción estimada.

En cuanto a la dispersión en la respuesta, fue notable al considerar las variables de la precipitación en el período de alta cobertura, mientras que lo contario se observó para el período de media a baja cobertura (Figuras 4.33 y 4.34). Nuevamente, la mayor homogeneidad de condiciones durante el período de media a baja cobertura con respecto al de alta cobertura discutida en la sección 4.3.1.b. estaría vinculada a tales diferencias.

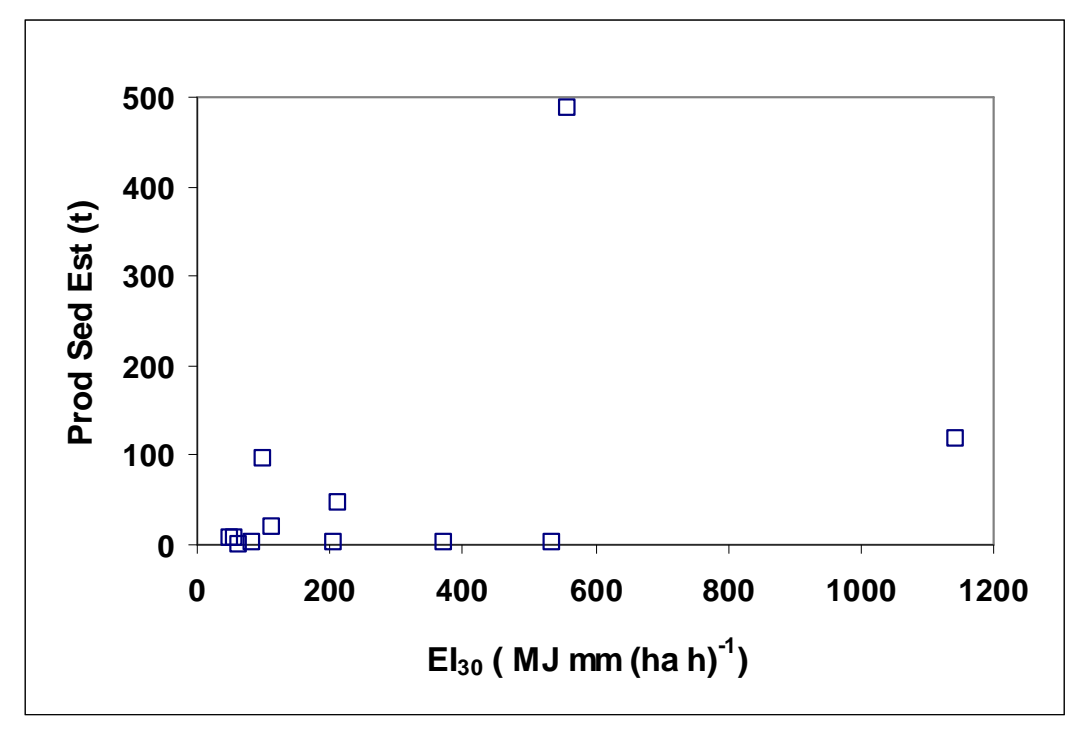

Figura 4.33. Relación entre la producción de sedimentos estimada y el índice $\mathrm{El}_{30}$ para el período de alta cobertura. 


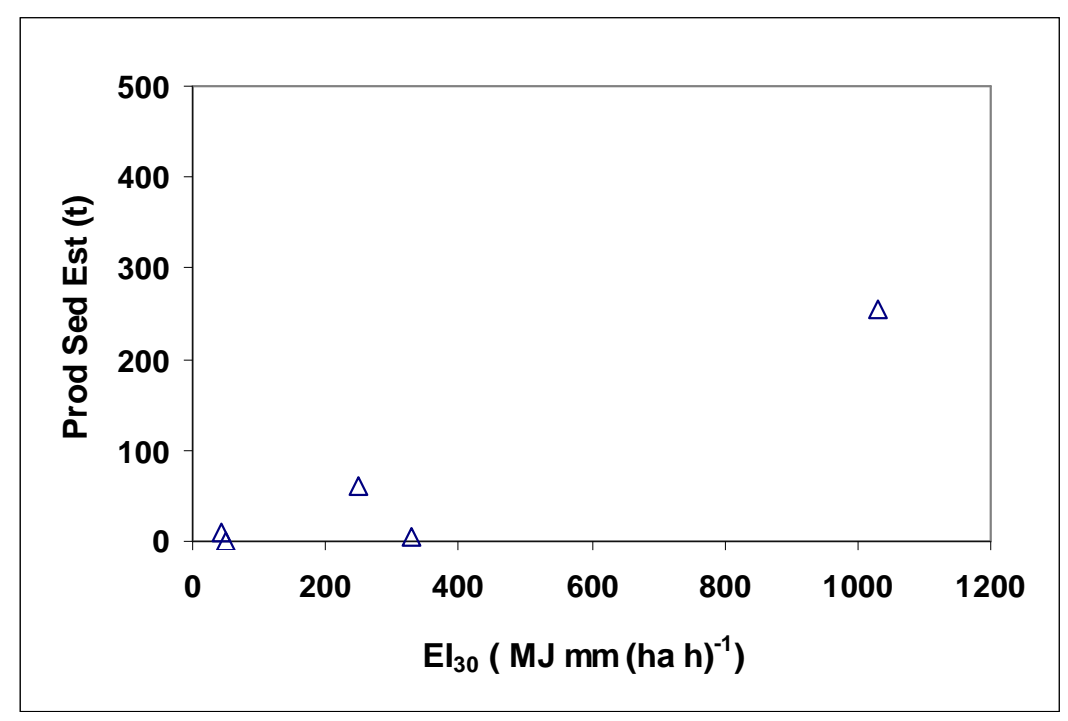

Figura 4.34. Relación entre la producción de sedimentos estimada y el índice $\mathrm{El}_{30}$ para el período de media a baja cobertura.

\subsection{Estimaciones de la producción de sedimentos a partir del modelo MUSLE}

Con la finalidad de evaluar los cálculos realizados de la producción total de sedimentos en los eventos mediante el modelo MUSLE, se compararon los valores obtenidos con los correspondientes a la producción de sedimentos estimada a partir de datos observados de concentración. La relación entre estas variables (Figura 4.35) presentó un buen ajuste estadístico $\left(R^{2}=0,59\right)$ y significancia al nivel $p<0,05$. Sin embargo, al comparar la tendencia de los datos con la recta de la relación 1 a 1 , se observa que los valores del modelo resultaron en casi todos los casos superiores a los estimados a partir de las mediciones realizadas en el área de estudio, aun teniendo en cuenta que estos últimos serían superiores a los que posiblemente hayan sido generados en los eventos (sección 4.5). Estas sobreestimaciones fueron mayores para los casos de los eventos número 1 (15/01/2011), 9 (17/05/2012) y 10 (23/08/2012). En tanto, en solo un caso el modelo subestimó la producción de sedimentos, y que correspondió al registrado el 19/12/2012. 


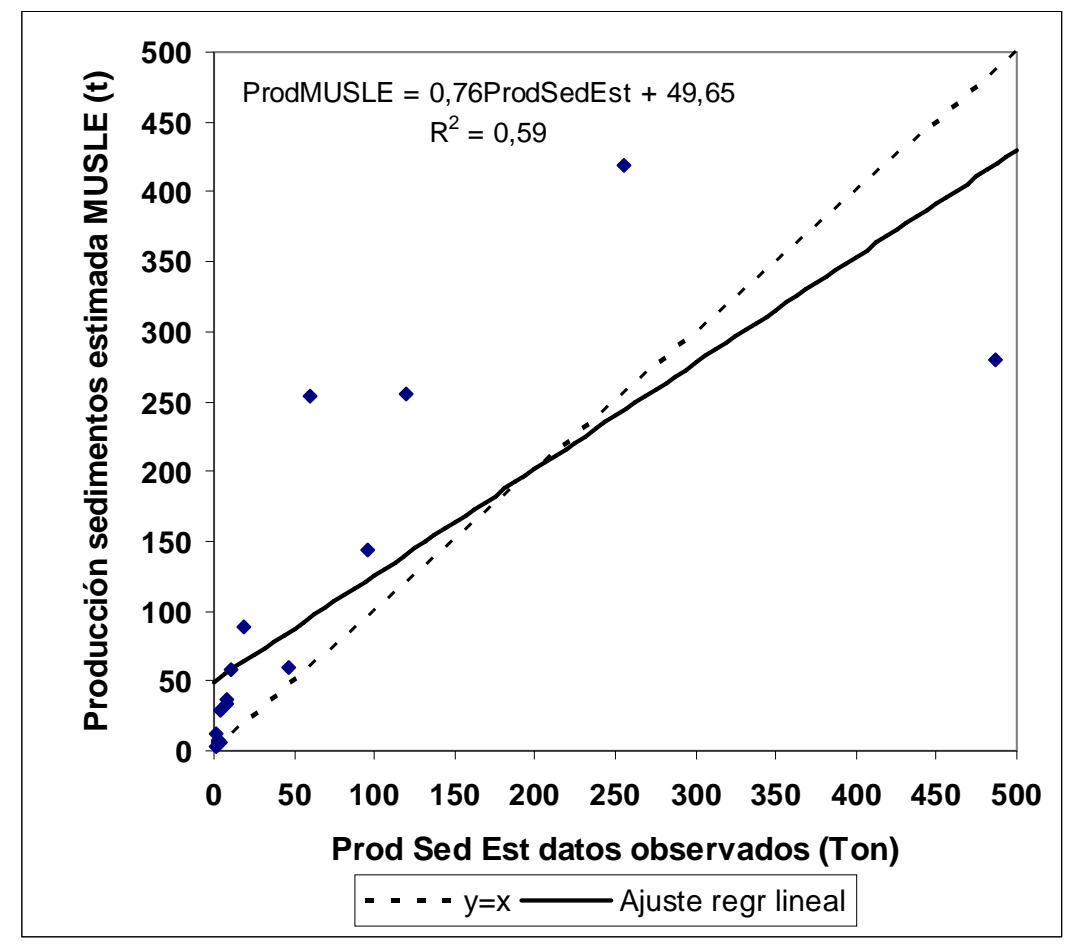

Figura 4.35. Relación entre los valores de producción de sedimentos calculados a través del modelo MUSLE y los valores de producción de sedimentos estimados a partir de los datos de concentración observados. Línea de ajuste a modelo de regresión lineal y línea teórica que describe la relación 1:1 entre las variables.

Cabe entonces analizar las posibles causas de tales diferencias con los valores modelados. En primer lugar, se podría postular que la producción de sedimentos en la microcuenca haya resultado escasa. Esto estaría asociado a las características de la microcuenca en cuanto a las propiedades de sus suelos, que, como se expuso anteriormente, presentaron contenidos de materia orgánica entre 6 y $7 \%$, y muy buenas estructuras porosas y estables, reflejado en los valores de estabilidad de estructura (Tabla 4.6). De tal modo, sería posible que el modelo no estuviera evaluando correctamente al factor de erodabilidad del suelo, el que sería menor que el estimado en este caso. Piscitelli et al. (2010) calcularon valores del factor para suelos de la cuenca superior del arroyo del Azul, en la que se encuentra la microcuenca, y obtuvieron valores comparables a los de la presente tesis. Los autores reportaron coeficientes de determinación de 0,1, para las regresiones entre los valores de $\mathrm{K}$ de los sitios que estudiaron y los de materia orgánica que les correspondían. Así, vincularon el bajo ajuste a los altos contenidos de materia orgánica, que no son contemplados en el cálculo del factor, ya que el nomograma permite ingresar un máximo de 4\%. Estos resultados entonces darían sustento a lo que fue postulado anteriormente, y así debido a la sobreestimación del 
factor de erodabilidad el modelo no permitiría evaluar de manera ajustada la producción de sedimentos.

Otro aspecto a tener en cuenta asociado a las propiedades de los suelos es su capacidad de infiltración. La misma se reflejaría, por un lado, en el valor de equilibrio de CN cercano a 62 discutido en la sección 4.3.1.a. A modo de comparación, se puede hacer mención al valor de CN que Chagas et al. (2008) calcularon, empleando la misma metodología, para una microcuenca agrícola de Pampa Ondulada, que fue de 82. Los suelos de esa región se caracterizan por sus texturas franco limosas y contenidos promedio de materia orgánica menores, de $4 \%$, con estructuras de menor estabilidad y menores valores de hidrofibicidad que los citados para la zona de trabajo de la presente tesis. Por lo tanto, las propiedades de estos suelos explicarían la menor capacidad de infiltración que se evidenció en el CN obtenido.

Por otro lado, en ensayos de simulación de lluvia realizados en la microcuenca del arroyo Videla (Ares et al., 2013) fue necesario aplicar intensidades de lluvia de $120 \mathrm{~mm} \mathrm{~h}^{-1}$ para lograr escurrimientos medibles en la primera hora de ensayo, bajo suelo cubierto y descubierto. Esto también resulta indicativo de esta propiedad de los suelos que contribuye a reducir los escurrimientos y como consecuencia la producción de sedimentos.

En segundo lugar, es posible mencionar a la tasa de liberación de sedimentos (o sediment delivery ratio, SDR) como otro de los factores involucrados en las diferencias resultantes. Como es conocido, no todo el material desprendido en las laderas de una cuenca llega al cauce. Esa proporción de dicho material que alcanza la salida de la cuenca se conoce como tasa de liberación de sedimentos (Morgan, 2005). En términos generales, según las características de las cuencas en cuanto a su relieve, pendientes, cobertura vegetal, textura de sus suelos, entre otras, esta tasa puede ser más alta en casos en los que la mayor parte del material desprendido llega al cauce y a la salida. Por el contrario, puede ser más baja en condiciones en las cuales el material desprendido se deposita en reservorios, o por diferentes barreras, como la vegetación (Walling, 1994). De acuerdo con las diferencias obtenidas en este caso bajo estudio, sería posible considerar que la microcuenca podría tener un valor bajo de esta tasa, en relación a diferentes factores. Uno de ellos, podría ser el predominio de procesos erosivos laminares, ya que la erosión en surcos, según lo discutido anteriormente, correspondería a una menor proporción de eventos. Así, por el tipo de proceso dominante sería menor la emisión de sedimentos, tal como indican Morgan (2005) y Cerdan et al. (2002) frente al proceso en surcos o en cárcavas. 
Otro factor es la cobertura vegetal, que se mantuvo al menos en una proporción de $50 \%$ durante todo el período, lo que habría sido favorable para el control del desprendimiento. Además, Beuselinck et al. (2000) mencionan la disminución en la tasa de entrega de sedimentos con la presencia de franjas de vegetación que actúan como barreras para la llegada del material al cauce. En este sentido, cabe comentar que el cauce de la microcuenca posee una ribera cubierta con vegetación densa durante todo el año, en la que no se realizan cultivos (Figura 4.36), la que actuaría como filtro o franja buffer pudiendo contribuir en la disminución del aporte de sedimentos al cauce.

Al mismo tiempo, y asociado a la posible producción escasa de sedimentos ya comentada, la textura del suelo y sus propiedades habrían aportado sus beneficios en cuanto a la tasa de entrega de sedimentos.

De este modo, se considera que las diferencias entre los valores modelados y los correspondientes a las estimaciones de producción de sedimentos a partir de datos observados estarían asociadas a factores relacionados con las características y propiedades de los suelos, que posiblemente no sean contempladas de manera ajustada por el modelo. Asimismo, tales propiedades en interacción con la cobertura vegetal podrían propiciar condiciones para el predominio de erosión de tipo laminar, el desprendimiento se vería limitado, y la entrega de sedimentos no sería tan alta como la estimada por parte de MUSLE.

Otro aspecto a mencionar está vinculado con los casos de mayores diferencias entre los valores predichos por el modelo y los estimados a través de las mediciones realizadas, los cuales fueron señalados al inicio de esta sección. Estos pertenecen a eventos que fueron relacionados con el proceso de erosión en surcos, y tres de ellos son aquellos que evidenciaron los cambios más importantes en la producción de sedimentos estimada con respecto al caudal pico, según lo analizado en el apartado 4.5.1.a. Esto permitiría establecer que la respuesta del sistema ante estos eventos resultaría más compleja y el modelo MUSLE presentaría más limitaciones para predecir la producción de sedimentos de manera más ajustada. Es decir que estos casos, que constituyen una menor proporción con respecto a la totalidad de los registrados pero que serían los responsables de la mayor pérdida de suelo en estos ambientes, presentarían las mayores dificultades para ser modelados.

Con respecto a todo lo analizado en este punto y a lo discutido en la sección 4.5.1.a, es posible señalar que el caudal pico sería una variable que permitiría estimar de manera más 
ajustada la producción de sedimentos con respecto al modelo MUSLE, para casos de eventos que se encuentren en el rango de los valores analizados en este trabajo, lo que se sustenta también en lo estudiado por Duvert et al. (2012) en cuencas de distintas áreas geográficas.

Por último, cabe recordar que la ecuación MUSLE es empleada en la estimación de sedimentos por parte de varios modelos de amplia difusión que evalúan el efecto de estrategias de manejo en la producción agrícola y en los recursos agua y suelo a nivel de cuenca tales como EPIC y SWAT (Williams \& Arnold, 1997; Nearing et al., 2005). Es así que estos resultados y su análisis pueden resultar de interés ya que orientan acerca de posibles sobreestimaciones y subestimaciones que podrían ser obtenidas al momento de la implementación de dichos modelos en el área de estudio y en otras de características comparables a las de esta región.

a)

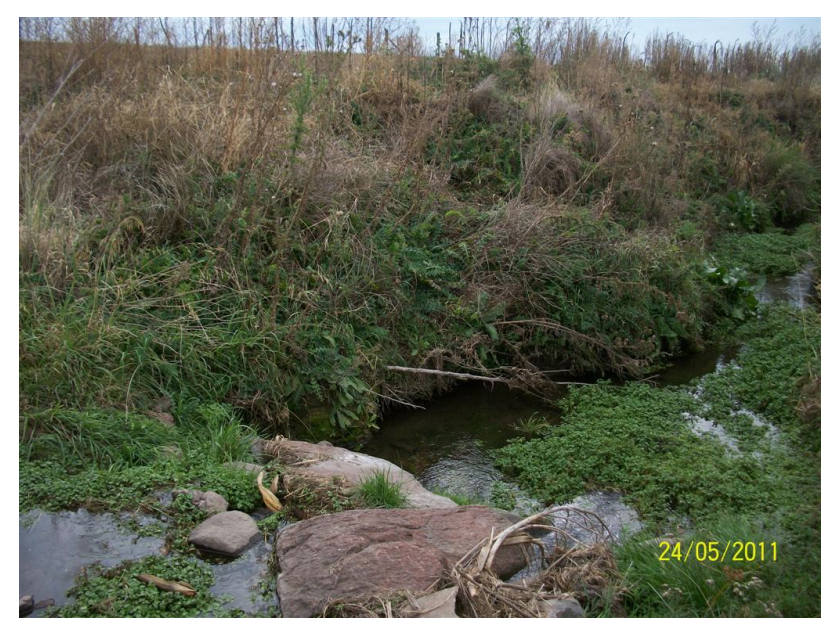

b)

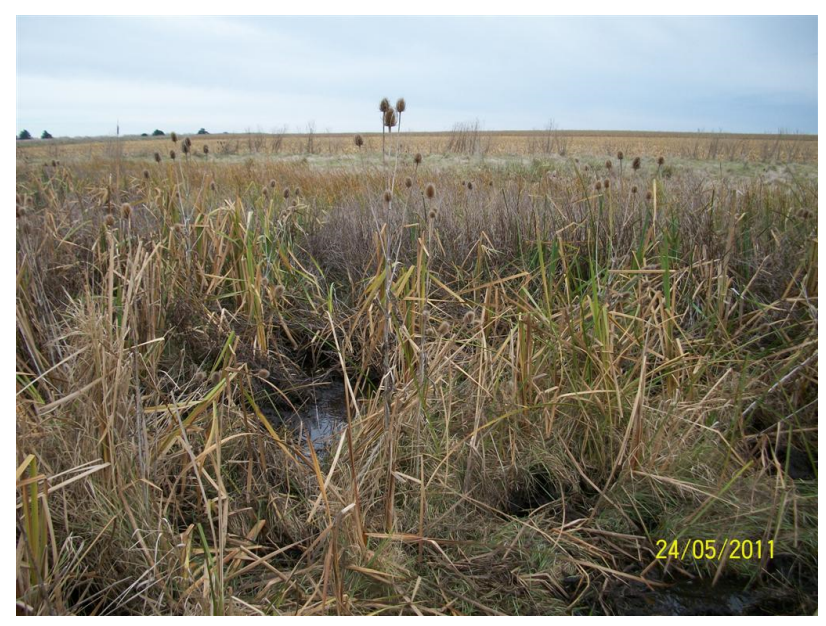

Figura 4.36 a) y b). Imágenes correspondientes a dos sectores de la ribera del curso de agua de la microcuenca bajo estudio, mostrando la zona de vegetación densa. 


\section{CONCLUSIONES}

Hipótesis 1:

En el área bajo estudio existe relación entre las características de las tormentas, generación de escurrimiento superficial y producción de sedimentos

\section{Conclusión:}

Varios de los aspectos discutidos en este trabajo permitirían corroborar esta hipótesis propuesta:

En términos generales, las variables asociadas al escurrimiento y a la precipitación se encontraron correlacionadas al considerar el conjunto de los eventos. La relación escurrimiento vs $\mathrm{El}_{30}$ presentó alta significancia estadística, por lo que esta variable sería adecuada para la predicción del escurrimiento. Asimismo, la precipitación, una variable de medición más sencilla y que no requiere de un procesamiento mayor de los datos, podría ser utilizada como predictora del escurrimiento total con buen ajuste.

Tanto la CSS del muestreo discreto como la del muestreo compuesto se relacionaron estadísticamente con variables asociadas al escurrimiento. Sin embargo, las vinculaciones significativas con las variables de la precipitación se registraron mayormente con la CSS del muestreo discreto.

La dispersión registrada en las relaciones entre la concentración de sólidos suspendidos del muestreo discreto y las variables asociadas al escurrimiento indicarían que el proceso erosivo en la microcuenca posee un comportamiento no lineal de alta variabilidad temporal. Las características de las precipitaciones en cuanto su estacionalidad, que condiciona su intensidad y erosividad, tendrían influencia en tal variabilidad, lo que habría quedado evidenciado a través de las relaciones entre la CSS discreta y las variables $I_{30}, I_{10}$ y $\mathrm{I}_{30}$, que presentaron significancia estadística.

En cuanto a la CSS de muestreo compuesto, los modelos exponenciales describieron de manera más adecuada las relaciones con las variables asociadas al escurrimiento, en tanto que fue posible identificar valores orientativos a partir de los cuales se registrarían incrementos importantes en la concentración compuesta. 
Al considerar las estimaciones de la producción de sedimentos totales para los eventos estudiados se encontraron relaciones entre esta variable e $\mathrm{I}_{30}$ y $\mathrm{El}_{30}$. Tales relaciones presentaron menores coeficientes de determinación que aquellas con el Qp, Qsm, CE o el factor de escurrimiento, las que se ajustaron a ecuaciones exponenciales. El análisis en detalle de la relación entre la producción de sedimentos estimada y el caudal pico permitió identificar cambios en la respuesta del sistema asociados a los mecanismos de erosión predominantes en la microcuenca durante el período de estudio. Estos cambios podrían vincularse a modificaciones en las condiciones de equilibrio de la cuenca que se producirían superando un valor umbral a partir del cual el sistema vería superada su capacidad de resiliencia (se hará referencia a este aspecto con mayor profundidad en el punto 3-).

En cuanto a la aplicación del modelo MUSLE, los resultados obtenidos permitirían corroborar también parte de esta hipótesis. Este modelo considera a la escorrentía como uno de los factores que intervienen en el transporte de sedimentos en las cuencas. Los valores calculados de los sedimentos producidos mediante MUSLE mostraron buena correlación con aquellos estimados a partir de datos observados de concentración $\left(R^{2}=0,59\right)$. Sin embargo, cabe comentar que dichos valores calculados resultaron en casi todos los casos superiores a los estimados a partir de las mediciones realizadas en el área de estudio. Si bien los parámetros de esta ecuación fueron obtenidos a través del procesamiento de datos correspondientes a las condiciones locales, las particularidades del área de estudio, en cuanto al alto contenido de materia orgánica de sus suelos, no estarían contempladas en el factor de erodabilidad. Así, sería posible que el modelo no estuviera evaluando correctamente al factor de erodabilidad del suelo, el que sería menor que el estimado en este caso. En tanto, otras propiedades de los suelos como la alta capacidad de infiltración contribuiría a reducir los escurrimientos y como consecuencia, la producción de sedimentos.

Además, la tasa de liberación de sedimentos podría haber resultado baja en relación a diferentes factores: el predominio de erosión de tipo laminar frente a erosión en surcos, la preponderancia de suelo cubierto en proporción superior a $50 \%$ en el período estudiado, o también la presencia de vegetación densa permanente en la ribera del cauce que actuaría como filtro o franja buffer pudiendo contribuir en la disminución del aporte de sedimentos al cauce.

De este modo, las diferencias entre los valores modelados y los correspondientes a las estimaciones de producción de sedimentos a partir de datos observados estarían asociadas a factores relacionados con las características y propiedades de los suelos, que 
posiblemente no sean contempladas de manera ajustada por el modelo. Asimismo, tales propiedades en interacción con la cobertura vegetal podrían propiciar condiciones para el predominio de erosión de tipo laminar, el desprendimiento se vería limitado, y la entrega de sedimentos no sería tan alta como la estimada por parte de MUSLE.

\section{Hipótesis 2:}

El escurrimiento y la producción de sedimentos sufren modificaciones a lo largo del año en función de la estacionalidad de las tormentas y de la variación en el uso del suelo

\section{Conclusión:}

El análisis de los datos considerando la estacionalidad en las precipitaciones y la cobertura permitió corroborar distintos aspectos de esta hipótesis. En primer lugar, fue posible identificar diferencias en cuanto a los factores que están involucrados en la respuesta lluviaescurrimiento-concentración y producción de sedimentos en períodos de alta cobertura y de media a baja cobertura. Así, para el primero de los períodos mencionados, la intensidad máxima de las precipitaciones en 30 minutos fue la variable de la precipitación asociada al escurrimiento. Esto sería indicativo de preponderancia de flujo superficial hortoniano como mecanismo de generación de escurrimiento en este caso. En cuanto a la concentración de sedimentos, se correlacionó con $I_{30}$ y además con las variables asociadas al escurrimiento, lo que indica que $I_{30}$ sería el factor preponderante que movilizaría al flujo y en consecuencia este último también a los sedimentos de las laderas.

En tanto, para el período de media a baja cobertura, las variables precipitación, duración de la precipitación, energía de la precipitación y la reserva de agua en el suelo se relacionaron con el escurrimiento y las variables asociadas, por lo que durante este período los mecanismos de flujo subsuperficial e infiltración desde superficie podrían producir la saturación en el suelo y generar el escurrimiento superficial. Al mismo tiempo, la correlación entre la intensidad máxima de la precipitación en 10 minutos y las variables vinculadas al escurrimiento indicaría que el mecanismo de Horton también se habría producido, probablemente en alguna tormenta de alta intensidad.

En cuanto a la concentración de sólidos suspendidos, durante este período se habría producido en respuesta directa a la precipitación, ya que únicamente las variables lluvia, energía, $\mathrm{I}_{30}$ y $\mathrm{El}_{30}$ se asociaron a tal concentración. 
Por otra parte, este análisis permitió diferenciar la variabilidad estacional en las respuestas al escurrimiento y a la producción de sedimentos. Durante la etapa de media a baja cobertura la homogeneidad en las condiciones de humedad, evaporación y la presencia predominante de rastrojos, se habría reflejado en una menor variabilidad en las respuestas mencionadas. Mientras tanto, en el otro período considerado la dispersión en la respuesta fue mayor, lo que se relacionó a la heterogeneidad en la cobertura de la microcuenca. Dicha heterogeneidad, sumada a la variabilidad en las características de las precipitaciones, en cuanto a su magnitud e intensidad, habrían contribuido a la variabilidad en la reserva de agua en el suelo, y así a la dispersión en la respuesta durante la etapa de alta cobertura.

El agrupamiento estacional permitió describir con más detalle los procesos involucrados en la generación de escurrimiento y producción de sedimentos, y evidenciar la acción diferencial que las lluvias y la cobertura ejercen, en relación a su dinámica temporal.

\section{Hipótesis 3:}

El proceso de erosión laminar resulta predominante frente al de erosión en surcos

\section{Conclusión:}

El análisis de los eventos registrados durante el período estudiado indicó que el proceso de erosión laminar fue el que se produjo con mayor frecuencia en la microcuenca, lo que permitiría corroborar la hipótesis propuesta. En tanto, otros aspectos en relación a los procesos en el área bajo estudio pudieron ser reconocidos. Entre ellos, se puede mencionar que la erosión en surcos tuvo lugar, pero en una menor proporción de eventos. En cuanto a la producción de sedimentos, los eventos asociados a erosión de tipo laminar fueron los que registraron los menores valores, mientras que aquellos relacionados con erosión en surcos fueron los que presentaron las mayores concentraciones y producciones estimadas. Se podría establecer entonces, que las mayores pérdidas se producirían en una cantidad de eventos reducida, que resultan de menor frecuencia.

Además, el estudio de la relación entre la producción de sedimentos estimada y el caudal pico permitió profundizar la caracterización del funcionamiento de este sistema ante los diferentes eventos, e identificar valores que podrían orientar acerca de cambios en los procesos predominantes, de erosión laminar a erosión en surcos, como asimismo, acerca de modificaciones en la intensidad del proceso en surcos. Este último aspecto resultó destacable, y tal modificación en la intensidad se vincularía a una posible pérdida de la capacidad de regulación de la cuenca, esto es, de la resiliencia de este sistema de alta 
capacidad productiva. Es decir, que la cuenca podría expresar tal capacidad de regulación, que estaría dada por las propiedades de sus suelos de alto contenido de materia orgánica, estabilidad de su estructura, que favorecerían la alta capacidad de infiltración, hasta cierto valor umbral de caudal pico en este caso. Al superarse dicho valor otros factores vinculados al flujo y su energía, los caminos preferenciales para el escurrimiento y su conectividad en el paisaje tendrían mayor incidencia en la producción de sedimentos y conducirían a las modificaciones de las condiciones de equilibrio del sistema, que implicarían asimismo, una alta salida de sedimentos.

Por su parte, los eventos por encima del valor umbral coincidieron con aquellos cuyos valores predichos mediante modelo de uso internacional MUSLE presentaron las mayores diferencias con respecto a aquellos que fueron estimados a partir de datos observados. Si bien la aplicación de este modelo, en términos generales, produjo sobreestimaciones de la producción de sedimentos, las mayores diferencias obtenidas para estos casos serían indicativas, por un lado, de la complejidad de la respuesta a la producción de sedimentos en estos eventos, y por otro lado de las mayores limitaciones de este modelo para las predicciones en estos casos. 


\section{BIBLIOGRAFÍA CITADA}

Allen, R.G., L.S. Pereira, D. Raes \& M. Smith. 1998. Crop evapotranspiration guidelines for computing crop water requeriments. FAO. Irrigation and Drainage Paper N56. 300 pp.

Ares, M.G., M. Varni, E. Usunoff \& L. Vives. 2005. Cálculo del número de curva en subcuencas del arroyo del Azul, provincia de Buenos Aires. Cuadernos del Curiham 11(1): 39-47.

Ares, M.G. 2010. Escurrimiento superficial en una cuenca del Sistema de Tandilia: análisis de su dinámica y de los principales factores que lo condicionan. M. Sc. Tesis Escuela para Graduados Alberto Soriano, Facultad de Agronomía, Universidad de Buenos Aires, Argentina. 88 pp.

Ares, M.G, M. Varni \& I. Entraigas. 2010. Determinación de la erosividad de las precipitaciones para la localidad de Azul, provincia de Buenos Aires, Argentina. En: Hacia la Gestión Integral de los Recursos Hídricos en Zonas de Llanura. Varni et al., Eds. Ed. Martín. Argentina. Tomo I, pp 95-99.

Ares, M.G., C. Chagas \& M. Varni. 2012a. Predicción de la escorrentía y estimación de la humedad antecedente en una cuenca aforada. Ciencia del Suelo 30(2): 151-160.

Ares, M.G., M. Varni., C. Chagas \& I. Entraigas. 2012b. Calibración del número N de la curva de escurrimiento en una cuenca agropecuaria de $116 \mathrm{~km}^{2}$ de la provincia de Buenos Aires, Argentina. Agrociencia (México) 46: 535-541.

Ares M.G., C. Chagas \& M. Varni. 2013. Comparación de la respuesta hidrológica de dos microcuencas con cultivos anuales bajo siembra directa ubicadas en geoambientes contastantes entre si (Pampa Ondulada y Pampa Serrana), en términos de Número de Curva de escurrimiento. Actas de Jornadas Argentinas de Conservación de Suelos. Buenos Aires, publicadas en CD.

Armand, R., C. Bockstaller, A.-V. Auzet \& P. Van Dijk. 2009. Runoff generation related to intra-field soil surface characteristics variability. Application to conservation tillage context. Soil \& Tillage Research 102: 27-37. 
ASTM Standard D3977-97. 2007. Test Methods for Determining Sediment Concentration in Water Samples. ASTM International, West Conshohocken, PA, USA.

Besteiro, S.I. \& F.J. Gaspari. 2012. Modelización de la emisión de sedimentos en una cuenca con forestaciones del Noreste Pampeano. Revista FCA UNCUYO 44(1): 111-127.

Beuselinck, L., A. Steegen, G. Govers, J. Nachtergaele, I. Takken \& J. Poesen. 2000. Characteristics of sediment deposits formed by intense rainfall events in small catchments in the Belgian Loam Belt. Geomorphology 32: 69-82.

Bilotta, G.S., T. Krueger, R. E. Brazier, P. Butler, J. Freer, J. M. B. Hawkins, P. M. Haygarth, C. J. A. Macleode \& J. N. Quintonc. 2010. Assessing catchment-scale erosion and yields of suspended solids from improved temperate grassland. Journal of Environmental Monitoring 12: 731-739.

Blanco, N. \& R. Lal. 2008. Principles of Soil Conservation and Management. Springer 617 pp.

Blöschl, G. \& M. Sivapalan. 1995. Scale issues in hydrological modelling: a review Hydrological Processes 9: 251-290.

Bouyoucos, G.J. 1962. Hydrometer methods improved for making particle size analysis of soils. Agronomy Journal 54: 464-465.

Böhm, P.\& G. Gerold. 1995. Pedo-hydrological and sediment responses to simulated rainfall on soils of the Konya Uplands (Turkey). Catena 25: 63-76

Brady, N. \& R.Weil. 2008. The nature and properties of soils. Pearson Prentice Hall. USA. $975 \mathrm{pp}$.

Burgos, J.J. \& J.A. Forte Lay. 1978. Capacidad de almacenaje de agua en los suelos de la región pampeana. En: Taller argentino-estadounidense sobre sequías (CONICET). Burgos J.J., Ed. Mar del Plata, Argentina. Pp 152-170.

Cammeraat, E.L. 2004. Scale dependent thresholds in hydrological and erosion response of a semi-arid catchment in southeast Spain. Agriculture, Ecosystems and Environment 104: 317-332. 
Casas, R. 2000. La Conservación de los Suelos y la Sustentabilidad de los Sistemas Agrícolas. Disertación. Disponible en: http://anterior.inta.gov.ar/suelos/actualidad/ conferencias/Disertacion R Casas.htm. Último acceso: Julio de 2013.

Casas, R. 2006. Presenvar la calidad y salud de los suelos: una oportunidad para la Argentina. Anales de la Academia Nacional de Agronomía y Veterinaria 60: 37-61.

Cazenave, G. 2006. Modelación numérica del escurrimiento superficial de la cuenca del arroyo del Azul: aplicación de modelos agregados y distribuidos. M. Sc. Tesis. Facultad de Ciencias Exactas, Ingeniería y Agrimensura, Universidad Nacional de Rosario, Argentina. $114 \mathrm{pp}$.

Cerdan, O., Y. Le Bissonnais, A. Coutorier, H. Bourennane \& V. Souchère. 2002. Rill erosion on cultivated hillslopes during two extreme rainfall events in Normandy, France. Soil \& Tillage Research 67: 99-108.

Cerdan, O., Y. Le Bissonnais, G. Govers, V. Lecomte, K. van Oost, A. Couturier, C. King, \& N. Dubreuil. 2004 Scale effect on runoff from experimental plots to catchment in agricultural areas in Normandy. Journal of Hydrology 299: 4-14.

Chagas, C.I., O.J. Santanatoglia, M.G. Castiglioni, M.J. Massobrio, A. Buján \& C. Irurtia. 2008. Número de Curva de escurrimiento para una microcuenca de Pampa Ondulada bajo labranza convencional y siembra directa. Ciencia del Suelo 26(1): 63-69.

Chapman, T. 1999. A comparison of algorithms for stream flow recession and baseflow separation. Hydrological Processes 13(5): 701-714.

Chenu, C., Y. Le Bissonnais \& D. Arrouays D. 2000. Organic matter influence on clay wettability and soil aggregate stability. Soil Science Society of America Journal 64: 14791486.

Chow, V.T., D. Maidment \& L. Mays. 1994. Hidrología aplicada. Mc Graw Hill. Santafé de Bogotá, Colombia. 584 pp.

Chuvieco, E. 2002. Teledetección ambiental. La observación de la Tierra desde el Espacio. Ed. Ariel S.A. Barcelona. 586 pp. 
Cisneros, J., C. Cholaky, A. Cantero Gutiérrez, J. González, M. Reynero, A. Diez \& L. Bergesio. 2012. Erosión hídrica: principios y técnicas de manejo. Ed. UniRío. Río Cuarto. $287 \mathrm{pp}$.

Collins, A.J. \& P.N. Owens. 2006. Introduction to soil erosion and sediment redistribution in river catchments: Measurement, modelling and management in the 21st century En: Soil erosion and sediment redistribution in river catchments measurement, modelling and management. Owens P.N \& A.J. Collins, Eds. CAB International, London, UK pp: 3-9.

Dalponte, D., P. Rinaldi, G. Cazenave, E. Usunoff, M. Varni, L. Vives, M.J. Vénere, \& A. Clausse. 2007. A validated fast algorithm for simulation of flooding events in plains. Hydrological Processes 21: 1115-1124.

da Silva, A.M. 2004. Rainfall erosivity map for Brazil. Catena 57: 251-257.

de Vente, J., J. Poesen, M. Arabkhedri \& G. Verstraeten. 2007. The sediment delivery problem revisited. Progress in Physical Geography 31(2): 155-178.

Dunne, T., T. Moore, \& C.H. Taylor. 1975. Recognition and prediction of runoff-producing zones in humid regions. Hydrological Sciences Bulletin 20(3): 305-327.

Duvert, C., N. Gratiot, O. Evrard, O. Navratil, J. Némery, C. Prat \& M. Esteves. 2010. Drivers of erosion and suspended sediment transport in three headwater catchments of the Mexican Central Highlands. Geomorphology 123: 246-256.

Duvert, C., G. Nord, N. Gratiot, O. Navratil, E. Nadal-Romero, N. Mathys, J. Némery, D. Regüés, J.M. García Ruiz, F. Gallart \& M. Esteves. 2012. Towards prediction of suspended sediment yield from peak discharge in small erodible mountainuos catchments $\left(0.45-22 \mathrm{~km}^{2}\right)$ of France, Mexico and Spain. Journal of Hydrology 454-455: 42-55.

Edwards, W.M., G.B. Triplett, D.M. Van Doren, L.B. Owens, C.E. Redmond \& W.A. Dick. 1993. Tillage studies with a corn-soybean rotation: Hydrology and sediment loss. Soil Science Society of America Journal 57(4): 1051-1055. 
Engel, F.L., I. Bertol, S.R. Ritter, A. Paz González, J. Paz-Ferreiro \& E. Vidal Vázquez. 2009. Soil erosion under simulated rainfall in relation to phenological stages of soybeans and tillage methods in Lages, SC, Brazil. Soil \& Tillage Research 103: 216-221.

Entraigas, I.\& N. Vercelli. 2012. Los paisajes de la cuenca del arroyo del Azul. Editorial Martin. 123 pp.

Entraigas, I., R. Rivas \& E. Usunoff. 2001. Intercepción y distribución de la precipitación bajo el canopeo de maíz. Revista Argentina de Agrometeorología 1(2): 89-94.

Esteves, M. \& J.M. Lapetite. 2003. A multiscale approach of runoff generation in a Shaelian gully catchment:a case study in Niger. Catena 50:255-271.

Estrany, J., C. García \& R.J. Batalla. 2009. Suspended sediment transport in a small Mediterranean agricultural catchment. Earth Surface Processes and Landforms 34: 929-940.

FAO. 2000. Manual de prácticas integradas de manejo y conservación de suelos. Boletín de Tierras y Aguas de la FAO № 8. Roma, Italia. 220 pp.

Feng, P. \& J.Z. Li. 2008. Scale effects on runoff generation in meso-scale and large-scale sub-basins in the Luanhe River Basin. Hydrology and Earth Sciences System Discussions 5: 1511-1531.

Gabriels, D., G. Ghekiere, W. Schiettecatte, \& I. Rottiers. 2003. Assesment of USLE cover-management C-factors for 40 crop rotation systems on arable farms in the Kemmelbeek watershed, Belgium. Soil \& Tillage Research 74: 47-53.

Gallart, F., P. Llorens, J. Latron \& D. Regüés. 2002. Hydrological processes and their seasonal controls in a small Mediterranean mountain catchment in the Pyrenees. Hydrology and Earth System Sciences 6(3): 527-537.

García-Ruiz, J.M., D. Regüés, B. Alvera, N. Lana-Renault, P. Serrano-Muela, E. NadalRomero, A. Navas, J. Latron, C. Martí-Bono \& J. Arnáez. 2008. Flood generation and sediment transport in experimental catchments affected by land use changes in the central Pyrenees. Journal of Hydrology 356: 245-260. 
Gaspari, F.J. 2005. Ordenamiento territorial de microcuencas en base al riesgo de erosión hídrica superficial a través de la aplicación de SIG. Revista electrónica de la REDLACH 1, año 2.

Gaspari, F.J., G.E. Senisterra, M.I. Delgado, A.M. Rodríguez Vagaría \& S.M. Besteiro. 2009. Manual de Manejo Integral de Cuencas Hidrográficas. 1ํedición, La Plata, 321 pp.

Gellis, A.C. 2013. Factors influencing storm-generated suspended-sediment concentrations and loads in four basins of contrasting land use, humid-tropical Puerto Rico. Catena 104: 3957.

Giménez, R., J. Casalí, I. Grande, J. Díez, M.A. Campo, J. Álvarez-Mozos \& M. Goñi. 2012. Factors controlling sediment export in a small agricultural watershed in Navarre (Spain). Agricultural Water Management 110: 1-8.

González-Hidalgo J.C., J.L. Peña-Monné \& M. de Luis. 2007. A review of daily soil erosion in Western Mediterranean areas. Catena 71: 193-199.

Gumiere, S.J., Y. Le Bissonnais \& D. Raclot. 2009. Soil resistance to interrill erosion: model parametrization and sensitivy. Catena 77: 274-284.

Hawkins, R. 1993. Asymptotic determination of runoff curve numbers from data. Journal of Irrigation and Drainage Engineering (ASCE) 119(2): 334-345.

Hjelmfelt, A.T. 1991. Investigaton of Curve Number procedure. Journal of Hydraulic Engineering ASCE 117(6): 725-737.

Holzman, M.E. 2013. Estimación del estrés hídrico en cultivos y su relación con rendimientos en la Región Pampeana mediante imágenes de temperatura de superficie e índice de vegetación. Tesis Doctoral en Geografía, Universidad Nacional del Sur, Bahía Blanca, Argentina. $104 \mathrm{pp}$.

IGM. Hoja 3760-22-1 "Base Azopardo" y Hoja 3760-22-3 "Chillar".

INDEC. 2010. Censo de población, viviendas y hogares por radios censales para la ciudad de Azul, Buenos Aires. Información en soporte digital. Instituto Nacional de Estadísticas y Censos de la República Argentina. 
INTA-CIRN. 1990. Atlas de Suelos de la República Argentina. Proyecto PNUD 85/019. Buenos Aires, Argentina. Tomo I, 731 pp.

INTA. 1992. Carta de Suelos de la República Argentina. Hoja 3760-22, Chillar.

Irurtia, C.B., R. Berón, O. Costamagna, O. \& A. Glave. 1996. Erosión, Provincia de Buenos Aires. En: El deterioro del ambiente en la Argentina. Fundación para la Educación, la Ciencia y la Cultura. Orientación Gráfica Editora. Buenos Aires pp: 55-64.

Jackson, W.L., B.P. Van Haveren \& K. Gebhardt. 1986. Use of the Modified USLE for average annual sediment yield estimates on small rangeland drainage basins. En: Drainage Basin Sediment Delivery. Proceedings of the Second Scientific Assembly of IAHS, Albuquerque. IAHS Publication № 159. Hadley R. F., Ed. IAHS Press, Institute of Hydrology, Wallingford,Oxfordshire, UK.

Jensen, J.R. 1996. Introductory Digital Image Processing. A remote sensing perspective. $2^{\text {nd }}$ edition. Prentice Hall. New Jersey. USA. 318 pp.

Jin, H., L. Hongwen, R. G. Rasaily, W. Qingji, C. Guohua, S. Yanbo, Q. Xiaodong \& L. Lijin. 2011. Soil properties and crop yields after 11 years of no tillage farming in wheat-maize cropping system in North China Plain. Soil \& Tillage Research 113: 48-54.

Kinnell, P.I. 2005. Why the Universal Soil Loss Equation and the revised version of it do not predict event erosion well. Hydrological Processes 19: 851-854.

Kinnell, P.I. 2007. Runoff dependent erosivity and slope length factors suitable for modelling annual erosion using the Universal Soil Loss Equation. Hydrological Processes 21: 26812689.

Kinnell, P.I. 2010. Event soil loss, runoff and the Universal Soil Loss Equation family of models: A review. Journal of Hydrology 385: 384-397.

Lal, R. 2001. Soil degradation by erosion. Land Degradation \& Development 12: 519-539.

Le Bissonnais, Y. 1996. Aggregate stability and assessment of soil crustability and erodability. I. Theory and metodology. European Journal of Soil Science 47: 425-437. 
Lenzi, M.A, \& L. Marchi. 2000. Suspended sediment load during floods in a small stream of the Dolomites (northeastern Italy). Catena 39: 267-282.

López-Tarazón, J.A., R.J. Batalla, D. Vercat \& J.C. Balasch. 2010. Rainfall, runoff and sediment transport relations in a mesosclae mountainous catchment: The River isábena (Ebro basin). Catena 82: 23-34.

Maalim, F., A. Melesse, P. Belmont \& K. Gran. 2013. Modeling the impact of land use changes on runoff and sediment yield in the Le Sueur watershed, Minnesota using GeoWEPP. Catena 107: 35-45.

Malet, J.-P., A.-V. Auzet, O. Maquaire, B. Ambroise, L. Descroix, M. Esteves, J.-P. Vandervaere \& E. Truchet. 2003. Soil surface characteristics influence on infiltration in black marls: application to the Super-Sauze earthflow (Southern Alps, France). Earth Surface Processes and Landforms 28: 547-564.

Manuel-Navarrete, D., G.C. Gallopín, M. Blanco, M. Díaz-Zorita, D.O. Ferraro, H. Herzer, P. Laterra, M.R. Murmis, G.P. Podestá, J. Rabinovich, E.H. Satorre, F. Torres \& E.F. Viglizzo. 2009. Multi-causal and integrated assessment of sustainability: the case of agriculturization in the Argentine Pampas. Environment, Development and Sustainability $11: 621-638$.

Marizza, M.S., R. Rapacioli, \& L. Vives. 2010. La problemática aluvional en el Alto Valle del Río Negro, Argentina. Tecnología y Ciencias del Agua 1(1): 21-34.

Mathys, N., S. Brochot, M. Meunier \& D. Richard. 2003. Erosion quantification in the small marly experimental catchments of Draix (Alpes Ahute Provence, France). Calibration of ETC. Rainfall-runoff-erosion model. Catena 50: 527-548.

Merritt, W.S., R.A. Letcher \& A.J. Jakeman. 2003. A review of erosion and sediment transport models. Environmental Modelling \& Software 18: 761-799.

Mishra, S.K. \& V. Singh. 2003. Soil Conservation Service Curve Number (SCS-CN) methodology. Kluwer Academic Publishers, Dordrecht, The Netherlands. 513 pp. 
Morgan, R.P. .2005. Soil Erosion and Conservation, $3^{\text {rd }}$ Edition. Blackwell Publishing. 304 pp.

Morris, N.L., P.C.H. Miller, J.H.Orson \& R.J. Froud-Williams. 2010.The adoption of noninversion tillage systems in the United Kingdom and the agronomic impact on soil, crops and the environment-A review. Soil \& Tillage Research 108: 1-15.

Mutchler, C.K., C.E. Murphree \& K.C. Mc Gregor. 1994. Laboratory and field plots for erosion research. En: Soil Erosion Research Methods. Lal, R., Ed. $2^{\text {nd }}$ Edition. SWCS, Ankeny, lowa. USA pp: 11-37.

Nadal Romero, E., D. Regüés \& J. Latron. 2008. Relationships among rainfall, runoff, and suspended sediment in a small catchment with badlands. Catena 74: 127-136.

Nearing, M.A., L.J. Lane \& V.L. Lopes. 1994. Modeling soil erosion. En: Soil Erosion Research Methods. Lal, R., Ed. $2^{\text {nd }}$ Edition. SWCS, Ankeny, lowa. USA pp: 127-156.

Nearing, M.A., V. Jetten, C. Baffaut, O. Cerdan, A. Couturier, M. Hernandez, Y. Le Bissonnais, M.H. Nichols, J.P. Nunes, C.S. Renschler, V. Souchère \& K. van Oost. 2005. Modeling response of soil erosion and runoff to changes in precipitation and cover. Catena 61: 131-154.

Nelson, D.W. \& L.E. Sommers. 1996. Total carbon, organic carbon, and organic matter. En: Methods of Soil Analysis-Part 3-Chemical methods. Sparks D.L et al., Ed. SSSA Inc. Madison, Wisconsin. USA pp: 961-1010.

Nu Fang, F., S. Zhi-Hua, L. Lu \& J. Cheng. 2011. Rainfall, runoff, and suspended sediment delivery relationships in a small agricultural watershed of the Three Gorges area, China. Geomorphology 135: 158-166.

Oeurng, C., S. Sauvage \& J.M Sánchez-Pérez. 2010. Dynamics of suspended sediment transport and yield in a large agricultural catchment, southwest France. Earth Surface Processes and Landforms 35: 1289-1301.

Onderka, M., A. Krein, S. Wrede, N. Martínez-Carreras \& L. Hoffmann. 2012. Dynamics of storm-driven suspended sediments in a headwater catchment described by multivariable modeling. Journal of Soils and Sediments 12:620-635. 
Owens, P.N., R.J. Batalla, A.J. Collins, B. Gómez, D.M. Hicks, A.J. Horowitz, G.M. Kondolf, M. Marden, M.J. Page, D.H. Peacock, E.L. Petticrew, W. Salomons \& N.A. Trustrum. 2005. Fine-grained sediment in river systems: environmental significance and management issues. River research and applications 21: 693-717.

Pandey, A., B.M. Chowdary \& C. Mal. 2009. Sediment yield modelling of an agricultural watershed using MUSLE, remote sensing and GIS. Paddy Water Environment 7: 105-113.

Pascale, J.A. \& E.A. Damario. 2004. Bioclimatología agrícola y Agroclimatología. Editorial Facultad de Agronomía. UBA. 550 pp.

Pilgrim, D.H. \& I. Cordery. 1993. Flood runoff. En:. Handbook of Hydrology. Maidment, D.R: Ed. McGraw-Hill Inc. New York, USA pp: 9.1-9.42.

Pimentel, D., C. Harvey, P. Resosudarmo, K. Sinclair, D. Kurz, M. McNair, S. Crist, L. Shpritz, L. Fitton, R. Saffouri \& R. Blair. 1995. Environmental and economic costs of soil erosion and conservation benefits. Science 267: 1117-1123.

Piscitelli, M., M. Varni, A. Sfeir \& M.G. Ares. 2010. Valores de erodabilidad (Factor KUSLE) para suelos representativos de la cuenca alta del arroyo Azul (Partido de AzulBuenos Aires). Revista de la Facultad de Agronomía, La Plata 109 (1): 17-22.

Polyakov, V.O., M.A. Nearing, M.H. Nichols, R.L. Scott, J.J. Stone \& M.P. McClaran. 2010. Long-term runoff and sediment yields from small semiarid watersheds in southern Arizona. Water Resources Research 46, W09512, doi:10.1029/2009WR009001.

Ponce, V.M. \& R. Hawkins. 1996. Runoff Curve Number: has it reached maturity? Journal of Hydrologic Engineering ASCE 1(1):11-19.

Renard, K.G., G.R. Foster, G.A. Weesies, D.K. Mc Cool, \& D.C Yoder (Coordinadores). 1997. Predicting soil erosion by water: a guide to conservation planning with the revised soil loss equation (RUSLE). USDA Agriculture Handbook № 703. US Government Printing Office, Washington DC, USA. 404 pp.

Ritter, D.F., R.C. Kochel \& J.R. Miller. 1999. The disruption of Grassy Creek: implications concerning catastrophic events and thresholds. Geomorphology 29: 323-338. 
Rivas, R. \& V. Caselles. 2004. A simplified equation to estimate spatial reference evapotranspiration from remote sensing-based surface temperature and local meteorological data. Remote Sensing of Environment 93:68-76.

Rivas, R. \& V. Caselles. 2005. Reference evapotranspiration in a pasture of Argentina. En: Recent Research Developments in Thermal Remote Sensing. Pandalai S.G., Ed. Kerala, India pp: 119-134.

Rodríguez L., C. Vionnet, G. Parkin \& P. Younger. 2000. Aplicación de un método automático para la separación de las componentes del hidrograma. Actas del XIX Congreso Latinoamericano de Hidráulica. Córdoba, Argentina. Tomo II pp. 279-286.

Rodríguez-Blanco, M.L., M.M. Taboada-Castro \& M.T. Taboada-Castro. 2010. Factors controlling hydro-sedimentary response during runoff events in a rural catchment in the humid Spanish zone. Catena 82: 206-217.

Sadeghi, S.H. \& T. Mizuyama. 2007. Applicability of the modified universal soil loss equation for prediction of sediment yield in Khanmirza watershed, Iran. Hydrolgical Sciences Journal 52(5): 1068-1075.

Schmidt, K-H. \& D. Morche. 2006. Sediment output and effective discharge in two small high mountain catchments in the Bavarian Alps, Germany. Geomorphology 80: 131-145.

Schowengerdt, R.A. 1997. Remote Sensing. Models and Methods for Image Processing. Academic Press, San Diego, USA. 522 pp.

Seeger, M., M.-P. Errea, S. Beguería, J. Arnáez, C. Martí \& J.M. García-Ruiz. 2004. Catchment soil moisture and rainfall characteristics as determinant factors for discharge/suspended sediment hysteretic loops in a small headwater catchment in the Spanish pyrenees. Journal of Hydrology 288:299-311.

Sfeir, A., M. Piscitelli, A. Sottile, R. Casas, R. Michelena; C. Irurtia, G.W.Thomas \& N. Díaz. 1998. Algunas propiedades del suelo en sistemas de siembra directa y labranza tradicional en áreas con altas pendientes de Azul. Actas XVI Congreso Argentino de la Ciencia del Suelo. Córdoba, Argentina p: 276. 
Sfeir, A., M. Varni, M. Piscitelli, R.J. Crespo, G. Ares \& G. Studdert. 2005. Cuantificación de los procesos de infiltración, escurrimiento y pérdida de suelo por medio de simuladores de lluvia. Actas de XX Congreso Nacional del Agua, Mendoza, publicado en CD.

Sfeir, A., M. Varni, M. Piscitelli, G. Ares, R.J. Crespo \& E. Usunoff. 2006. Efecto de la siembra directa permanente sobre la reducción del escurrimiento superficial. III Congreso Iberoamericano sobre el control de la erosión y los sedimentos, publicado en CD.

Shipitalo, M.J. \& W.M. Edwards. 1998. Runoff and erosion control with conservation tillage and reduced-input practices on cropped watersheds. Soil \& Tillage Research 46 1-12.

Smith, H. 2008. Estimation of suspended sediment loads and delivery in an incised Upland headwater catchment, south-eastern Australia. Hydrological Processes 22: 3135-3148.

Sidle, R.C., T. Hirano, T. Gomi \& T. Terajima. 2007. Hortonian overland flow from Japanese forest plantations-an aberration, the real thing, or something in between? Hydrological Processes 21: 3237-3247.

Silveira, L., F. Charbonnier \& J.L. Genta. 2000. The antecedent soil moisture condition of the curve number procedure. Hydrological Sciences Journal 45(1): 3-12.

Soulis, K.X., J.D. Valiantzas, N. Dercas \& P.A. Londra. 2009. Analysis of the runoff generation mechanism for the investigation of the SCS-CN method applicability to a partial area experimental watershed. Hydrology and Earth System Sciences 6: 373-400.

Smith, S.J., J.R. Williams, R.G. Menzel \& G.A. Coleman. 1984. Prediction of sediment yield from southern plains grasslands with the Modified Universal Soil Loss equation. Journal of Range Management 37(4): 295-297.

Soler, M., D. Regüés, J. Latron \& F. Gallart. 2007. Frequency-magnitude relationships for precipitation, stream flow and sediment load events in a small Mediterranean basin (Vallcebre basin, eastern Pyrenees). Catena 71:164-171.

Soler, M., J. Latron \& F.Gallart. 2008. Relationships between suspended sediment concentrations and discharge in two small research basins in a mountainous Mediterranean area (Vallcebre, Eastern Pyrenees). Geomorphology 98: 143-152. 
Steegen, A., G. Govers, J. Nachtergaele, I. Takken, L. Beuselinck \& J. Poesen. 2000. Sediment export by water from an agricultural catchment in the Loam Belt of central Belgium. Geomorphology 33: 25-36.

Terzoudi, B.C., T.A. Gemtos, N.G. Danalatos \& I. Argyrokastritis. 2007. Applicability of an empirical runoff estimation method in central Greece. Soil \& Tillage Research 92: 198-212.

Thornes, J.B. 1980. Procesos erosivos de las corrientes de agua y sus controles espaciales: un punto de vista teórico. En: Erosión de Suelos. M.J. Kirkby \& R.P.C. Morgan (coordinadores). Ed. Limusa. México pp 165-225.

Tramblay, Y., C. Bouvier, C. Martin, J.F. Didon-Lescot, D. Todorovik \& J.M. Domergue. 2010. Assessment of initial soil moisture conditions for event-based rainfall-runoff modeling. Journal of Hydrology 387: 176-187.

USDA SCS. 1985. National Engineering Handbook, Section 4. U.S. Government. Printing Office, Washington DC.

Valentin, C., J. Poesen \& Y. Li. 2005. Gully erosion: Impacts, factors and control. Catena 63: $132-153$.

van Dijk A.I., L.A. Bruijnzeel \& C.J. Rosewell. 2002. Rainfall intensity-kinetic energy relationships: a critical literature appraisal. Journal of Hydrology 261: 1-23.

Van Mullem J.A., R.H. Hawkins, A.T. Hjelmfelt \& Q.D. Quan. 2002. Runoff Curve Number method: Beyond the handbook. Second Federal Interagency Hydrologic Modelling Conference, Las Vegas.

Van Wie, B., J.C. Adam \& J.L. UlIman. 2013. Conservation tillage in dryland agriculture impacts watershed hydrology. Journal of Hydrology 483: 26-38.

Varela, C., I. Entraigas \& M. Varni. 2002. Aplicación del método de componentes principales a la determinación de regiones homogéneas de precipitación. Revista Argentina de Agrometeorología 2(1): 105-112. 
Varni, M., E. Usunoff, M. Gandini \& I. Entraigas. 1996. Simulación de crecidas en la cuenca del arroyo Azul, provincia de Buenos Aires. Primera Jornada de Desarrollo Regional en la Cuenca del Río Salado, Chacabuco, Provincia de Buenos Aires.

Varni, M. \& E. Custodio. 2013a. Estimación de la recarga al acuífero del Azul: 1. Análisis de las características climáticas. En: Agua subterránea recurso estratégico. N. González, E. Kruse, M. Trovatto \& P. Laurencena, Eds. Ed EDULP. La Plata. Tomo 1, pp: 31-37.

Varni, M. \& E. Custodio. 2013b. Estimación de la recarga al acuífero del Azul: 2. Balances de agua en el suelo y de deposición atmosférica de cloruro. En: Agua subterránea recurso estratégico. N. González, E. Kruse, M. Trovatto \& P. Laurencena, Eds. Ed EDULP. La Plata. Tomo 1, pp: 38-45.

Walling, D.E. 1994. Measuring sediment yield from river basins. En: Soil Erosion Research Methods. Lal, R., Ed. $2^{\text {nd }}$ Edition. SWCS, Ankeny, lowa. USA pp: 39-73.

Weinzettel, P.A. 2005. Hidrodinámica de la zona no saturada en suelos Argiudoles de la cuenca del Arroyo Azul. Tesis Doctoral en Geología, Universidad Nacional del Sur, Bahía Blanca, Argentina. $168 \mathrm{pp}$.

West, L.T., W.P. Miller, R.R. Bruce, G.W. Langdale, J.M. Laflen \& A.W. Thomas. 1992. Cropping system and consolidation effects on rill erosion in the Georgia Piedmont. Soil Science Society of America Journal 56: 1238-1243.

Williams, G.P. 1989. Sediment concentration versus water discharge during single hydrologic events in rivers. Journal of Hydrology 111(1): 89-106.

Williams, J.R. 1975. Sediment routing for agricultural watersheds. Water Resources Bulletin 11(5): 965-974.

Williams, J.R. \& H.D. Berndt. 1977. Sediment yield prediction based on watershed hydrology. Transactions of the ASAE 20(6): 1100-1104.

Williams, J.R. \& J.G. Arnold. 1997. A system of erosion-sediment yield models. Soil Technology 11: 43-55. 
Wischmeier, W.H. 1959. A rainfall erosion index for a universal soil loss equation. Soil Science Society of America Journal 23: 246-249.

Wischmeier, W.H., C.B. Johnson \& B.V. Cross. 1971. A soil erodibility nomograph for farmland and construction sites. Journal of Soil and Water Conservation 26: 189-193.

Wischmeier W.H. \& D.D. Smith. 1978. Predicting rainfall erosion losses-a guide to conservation planning. Handbook 537, USDA-ARS.

Zabaleta, A., M. Martínez, Uriarte, J.A. \& I. Antigüedad. 2007. Factors controlling suspended sediment yield during runoff events in small headwater catchments of the Basque Country. Catena 71: 179-190.

Zhang G.S., K.Y Chan, A. Oates, D.P. Heenan \& G.B. Huang. 2007. Relationship between soil structure and runoff/soil loss after 24 years of consenvation tillage. Soil \& Tillage Research 92: 122-128. 


\section{ANEXO}

Tabla 7.1. Planilla de cálculo del factor C. Detalle de fecha de la crecida, usos del suelo, etapa en la que se encontraban los cultivos y valor de relación de pérdida de suelo asociado para cada lote correspondiente a la microcuenca. Valor de $\mathrm{C}$ ponderado para la crecida.

\begin{tabular}{|c|c|c|c|c|c|c|}
\hline $\begin{array}{l}\text { Fecha } \\
\text { evento }\end{array}$ & Lote & $\begin{array}{c}\text { Superficie } \\
\text { del lote (ha) }\end{array}$ & Uso & $\begin{array}{c}\text { Etapa del } \\
\text { cultivo }\end{array}$ & $\begin{array}{c}\text { Valor relación } \\
\text { pérdida de suelo }\end{array}$ & $\begin{array}{c}\text { Factor } \mathrm{C} \\
\text { ponderado }\end{array}$ \\
\hline \multirow{8}{*}{$15 / 01 / 2011$} & 1 & 34,2 & Girasol & Período 3 & 0,11 & \multirow{8}{*}{0,11} \\
\hline & 2 & 26,7 & Soja $1^{a}$ & Período 3 & 0,07 & \\
\hline & 3 & 13,5 & Soja $2^{\underline{a}}$ & Período 1 & 0,08 & \\
\hline & 4 & 90,4 & Maíz & Período 3 & 0,11 & \\
\hline & 5 & 180,1 & Maíz & Período 3 & 0,11 & \\
\hline & 6 & 59,9 & Soja $1^{\text {a }}$ & Período 3 & 0,14 & \\
\hline & 7 & 108,1 & Soja de $1^{a}$ & Período 3 & 0,14 & \\
\hline & $\begin{array}{l}\text { Área cerros } \\
\text { y pajonales }\end{array}$ & 53,6 & - & - & 0,00003 & \\
\hline \multirow{8}{*}{$01 / 05 / 2011$} & 1 & 34,2 & Barbecho (rastrojo de girasol) & - & 0,4 & \multirow{8}{*}{0,20} \\
\hline & 2 & 26,7 & Soja 1a (precosecha) & Período 3 & 0,07 & \\
\hline & 3 & 13,5 & Soja $2^{2}$ (precosecha) & Período 3 & 0,07 & \\
\hline & 4 & 90,4 & Maíz (precosecha) & Período 3 & 0,11 & \\
\hline & 5 & 180,1 & Maíz (precosecha) & Período 3 & 0,11 & \\
\hline & 6 & 59,9 & Barbecho (rastrojo de soja $1^{a}$ ) & - & 0,4 & \\
\hline & 7 & 108,1 & Barbecho (rastrojo de soja $1^{a}$ ) & - & 0,4 & \\
\hline & $\begin{array}{l}\text { Área cerros } \\
\text { y pajonales }\end{array}$ & 53,6 & - & - & 0,00003 & \\
\hline \multirow{8}{*}{$08 / 11 / 2011$} & 1 & 34,2 & Cebada & Período 3 & 0,08 & \multirow{8}{*}{0,14} \\
\hline & 2 & 26,7 & Trigo & Período 3 & 0,07 & \\
\hline & 3 & 13,5 & Maíz & $\begin{array}{c}\text { Siembra- } 10 \% \\
\text { cobertura }\end{array}$ & 0,12 & \\
\hline & 4 & 90,4 & Soja $1^{a}$ & $\begin{array}{c}\text { Siembra- } 10 \% \\
\text { cobertura }\end{array}$ & 0,17 & \\
\hline & 5 & 180,1 & Barbecho (rastrojo de maíz) & - & 0,26 & \\
\hline & 6 & 59,9 & Cebada & Período 3 & 0,08 & \\
\hline & 7 & 108,1 & Cebada & Período 3 & 0,08 & \\
\hline & $\begin{array}{l}\text { Área cerros } \\
\text { y pajonales }\end{array}$ & 53,6 & - & - & 0,00003 & \\
\hline \multirow{8}{*}{$10 / 01 / 2012$} & 1 & 34,2 & Soja $2^{a}$ & Período 1 & 0,08 & \multirow{8}{*}{0,07} \\
\hline & 2 & 26,7 & Barbecho (rastrojo de trigo) & - & 0,08 & \\
\hline & 3 & 13,5 & Maíz & Período 3 & 0,08 & \\
\hline & 4 & 90,4 & Soja $1^{\text {a }}$ & Período 3 & 0,07 & \\
\hline & 5 & 180,1 & Soja $1^{a}$ & Período 3 & 0,07 & \\
\hline & 6 & 59,9 & Barbecho (rastrojo de cebada) & - & 0,08 & \\
\hline & 7 & 108,1 & Soja $2^{a}$ & Período 1 & 0,08 & \\
\hline & $\begin{array}{l}\text { Área cerros } \\
\text { y pajonales }\end{array}$ & 53,6 & - & - & 0,00003 & \\
\hline
\end{tabular}


Lluvia, escurrimiento y producción de sedimentos en una microcuenca agrícola del Sistema de Tandilia

Tabla 7.1 (cont). Planilla de cálculo del factor C. Detalle de fecha de la crecida, usos del suelo, etapa en la que se encontraban los cultivos y valor de relación de pérdida de suelo asociado para cada lote correspondiente a la microcuenca. Valor de C ponderado para la crecida.

\begin{tabular}{|c|c|c|c|c|c|c|}
\hline $\begin{array}{l}\text { Fecha } \\
\text { evento }\end{array}$ & Lote & $\begin{array}{c}\text { Superficie } \\
\text { del lote (ha) }\end{array}$ & Uso & $\begin{array}{l}\text { Etapa del } \\
\text { cultivo }\end{array}$ & $\begin{array}{c}\text { Valor relación } \\
\text { pérdida de suelo }\end{array}$ & $\begin{array}{c}\text { Factor C } \\
\text { ponderado }\end{array}$ \\
\hline \multirow{8}{*}{$11 / 03 / 2012$} & 1 & 34,2 & Soja $2^{a}$ & Período 3 & 0,07 & \multirow{8}{*}{0,07} \\
\hline & 2 & 26,7 & Barbecho (rastrojo de trigo) & - & 0,08 & \\
\hline & 3 & 13,5 & Maíz & Período 3 & 0,11 & \\
\hline & 4 & 90,4 & Soja $1^{\text {a }}$ & Período 3 & 0,07 & \\
\hline & 5 & 180,1 & Soja $1^{\text {a }}$ & Período 3 & 0,07 & \\
\hline & 6 & 59,9 & Barbecho (rastrojo de cebada) & - & 0,08 & \\
\hline & 7 & 108,1 & Soja $2^{\mathrm{a}}$ & Período 3 & 0,07 & \\
\hline & $\begin{array}{l}\text { Área cerros } \\
\text { y pajonales }\end{array}$ & 53,6 & - & - & 0,00003 & \\
\hline \multirow{8}{*}{$18 / 04 / 2012$} & 1 & 34,2 & Soja $2^{\mathrm{a}}$ & Período 3 & 0,07 & \multirow{8}{*}{0,09} \\
\hline & 2 & 26,7 & Barbecho (rastrojo de trigo) & - & 0,12 & \\
\hline & 3 & 13,5 & Maíz & Período 3 & 0,11 & \\
\hline & 4 & 90,4 & Barbecho (rastrojo de soja $1^{a}$ ) & - & 0,17 & \\
\hline & 5 & 180,1 & Soja 1aㅡ (precosecha) & Período 3 & 0,07 & \\
\hline & 6 & 59,9 & Barbecho (rastrojo de cebada) & - & 0,12 & \\
\hline & 7 & 108,1 & Soja $2^{a}$ & Período 3 & 0,07 & \\
\hline & $\begin{array}{l}\text { Área cerros } \\
\text { y pajonales }\end{array}$ & 53,6 & - & - & 0,00003 & \\
\hline \multirow{8}{*}{$17 / 05 / 2012$} & 1 & 34,2 & Soja $2^{\mathrm{a}}$ & Período 3 & 0,07 & \multirow{8}{*}{0,12} \\
\hline & 2 & 26,7 & Barbecho (rastrojo de trigo) & - & 0,12 & \\
\hline & 3 & 13,5 & Maíz & Período 3 & 0,11 & \\
\hline & 4 & 90,4 & Barbecho (rastrojo de soja) & - & 0,17 & \\
\hline & 5 & 180,1 & Barbecho (rastrojo de soja) & - & 0,17 & \\
\hline & 6 & 59,9 & Barbecho (rastrojo de cebada) & - & 0,12 & \\
\hline & 7 & 108,1 & Soja $2^{\mathrm{a}}$ & Período 3 & 0,07 & \\
\hline & $\begin{array}{l}\text { Área cerros } \\
\text { y pajonales }\end{array}$ & 53,6 & - & - & 0,00003 & \\
\hline \multirow{8}{*}{ 23/08/2012 } & 1 & 34,2 & Barbecho (rastrojo de soja $2^{a}$ ) & - & 0,18 & \multirow{8}{*}{0,15} \\
\hline & 2 & 26,7 & Barbecho (rastrojo de trigo) & - & 0,17 & \\
\hline & 3 & 13,5 & Barbecho (rastrojo de maíz) & - & 0,26 & \\
\hline & 4 & 90,4 & Cebada & Período 2 & 0,18 & \\
\hline & 5 & 180,1 & Barbecho (rastrojo de soja $1^{a}$ ) & - & 0,17 & \\
\hline & 6 & 59,9 & Barbecho (rastrojo de cebada) & - & 0,08 & \\
\hline & 7 & 108,1 & Barbecho (rastrojo de soja $2^{\underline{a}}$ ) & - & 0,18 & \\
\hline & $\begin{array}{l}\text { Área cerros } \\
\text { y pajonales }\end{array}$ & 53,6 & - & - & 0,00003 & \\
\hline
\end{tabular}


Tabla 7.1 (cont). Planilla de cálculo del factor C. Detalle de fecha de la crecida, usos del suelo, etapa en la que se encontraban los cultivos y valor de relación de pérdida de suelo asociado para cada lote correspondiente a la microcuenca. Valor de C ponderado para la crecida.

\begin{tabular}{|c|c|c|c|c|c|c|}
\hline $\begin{array}{l}\text { Fecha } \\
\text { evento }\end{array}$ & Lote & $\begin{array}{c}\text { Superficie } \\
\text { del lote (ha) }\end{array}$ & Uso & $\begin{array}{c}\text { Etapa del } \\
\text { cultivo }\end{array}$ & $\begin{array}{c}\text { Valor relación } \\
\text { pérdida de suelo }\end{array}$ & $\begin{array}{l}\text { Factor C } \\
\text { ponderado }\end{array}$ \\
\hline \multirow{8}{*}{ 03/09/2012 } & 1 & 34,2 & Barbecho (rastrojo de soja $2^{a}$ ) & - & 0,18 & \multirow{8}{*}{0,15} \\
\hline & 2 & 26,7 & Barbecho (rastrojo de trigo) & - & 0,17 & \\
\hline & 3 & 13,5 & Barbecho (rastrojo de maíz) & - & 0,26 & \\
\hline & 4 & 90,4 & Cebada & Período 3 & 0,1 & \\
\hline & 5 & 180,1 & Barbecho (rastrojo de soja $1^{a}$ ) & - & 0,17 & \\
\hline & 6 & 59,9 & Barbecho (rastrojo de cebada) & - & 0,17 & \\
\hline & 7 & 108,1 & Barbecho (rastrojo de soja $2^{\underline{a}}$ ) & - & 0,18 & \\
\hline & $\begin{array}{l}\text { Área cerros } \\
\text { y pajonales }\end{array}$ & 53,6 & - & - & 0,00003 & \\
\hline \multirow{8}{*}{ 05/10/2012 } & 1 & 34,2 & Barbecho (rastrojo de soja $2^{\underline{a}}$ ) & - & 0,17 & \multirow{8}{*}{0,14} \\
\hline & 2 & 26,7 & Barbecho (rastrojo de trigo) & - & 0,17 & \\
\hline & 3 & 13,5 & Barbecho (rastrojo de maíz) & - & 0,26 & \\
\hline & 4 & 90,4 & Cebada & Período 3 & 0,07 & \\
\hline & 5 & 180,1 & Barbecho (rastrojo de soja $1^{a}$ ) & - & 0,17 & \\
\hline & 6 & 59,9 & Barbecho (rastrojo de cebada) & - & 0,17 & \\
\hline & 7 & 108,1 & Barbecho (rastrojo de soja $2^{a}$ ) & - & 0,18 & \\
\hline & $\begin{array}{l}\text { Área cerros } \\
\text { y pajonales }\end{array}$ & 53,6 & - & - & 0,00003 & \\
\hline \multirow{8}{*}{$15 / 10 / 2012$} & 1 & 34,2 & Barbecho (rastrojo de soja $2^{a}$ ) & - & 0,18 & \multirow{8}{*}{0,17} \\
\hline & 2 & 26,7 & Barbecho (rastrojo de trigo) & - & 0,17 & \\
\hline & 3 & 13,5 & Barbecho (rastrojo de maíz) & - & 0,26 & \\
\hline & 4 & 90,4 & Cebada & Período 3 & 0,07 & \\
\hline & 5 & 180,1 & Maíz & $\begin{array}{l}\text { Siembra- } 10 \% \\
\text { cobertura }\end{array}$ & 0,25 & \\
\hline & 6 & 59,9 & Barbecho (rastrojo de cebada) & - & 0,17 & \\
\hline & 7 & 108,1 & Barbecho (rastrojo de soja $2^{\underline{a}}$ ) & - & 0,18 & \\
\hline & $\begin{array}{l}\text { Área cerros } \\
\text { y pajonales }\end{array}$ & 53,6 & - & - & 0,00003 & \\
\hline \multirow{8}{*}{$22 / 11 / 2012$} & 1 & 34,2 & Barbecho (rastrojo de soja $2^{a}$ ) & - & 0,18 & \multirow{8}{*}{0,13} \\
\hline & 2 & 26,7 & Barbecho (rastrojo de trigo) & - & 0,17 & \\
\hline & 3 & 13,5 & Barbecho (rastrojo de maíz) & - & 0,26 & \\
\hline & 4 & 90,4 & Cebada & Período 3 & 0,07 & \\
\hline & 5 & 180,1 & Maíz & Período 1 & 0,2 & \\
\hline & 6 & 59,9 & Soja $1^{\underline{a}}$ & $\begin{array}{c}\text { Siembra- } 10 \% \\
\text { cobertura }\end{array}$ & 0,09 & \\
\hline & 7 & 108,1 & Soja $1^{\underline{a}}$ & $\begin{array}{c}\text { Siembra- } 10 \% \\
\text { cobertura }\end{array}$ & 0,09 & \\
\hline & $\begin{array}{l}\text { Área cerros } \\
\text { y pajonales }\end{array}$ & 53,6 & - & - & 0,00003 & \\
\hline
\end{tabular}


Tabla 7.1 (cont). Planilla de cálculo del factor C. Detalle de fecha de la crecida, usos del suelo, etapa en la que se encontraban los cultivos y valor de relación de pérdida de suelo asociado para cada lote correspondiente a la microcuenca. Valor de C ponderado para la crecida.

\begin{tabular}{|c|c|c|c|c|c|c|}
\hline $\begin{array}{l}\text { Fecha } \\
\text { evento }\end{array}$ & Lote & $\begin{array}{c}\text { Superficie } \\
\text { del lote (ha) }\end{array}$ & Uso & $\begin{array}{l}\text { Etapa del } \\
\text { cultivo }\end{array}$ & $\begin{array}{c}\text { Valor relación } \\
\text { pérdida de suelo }\end{array}$ & $\begin{array}{l}\text { Factor C } \\
\text { ponderado }\end{array}$ \\
\hline \multirow{8}{*}{ 05/12/2012 } & 1 & 34,2 & Soja $1^{\underline{a}}$ & $\begin{array}{l}\text { Siembra- } 10 \% \\
\text { cobertura }\end{array}$ & 0,09 & \multirow{8}{*}{0,11} \\
\hline & 2 & 26,7 & Soja $1^{a}$ & $\begin{array}{l}\text { Siembra- } 10 \% \\
\text { cobertura }\end{array}$ & 0,09 & \\
\hline & 3 & 13,5 & Soja $1^{\underline{a}}$ & $\begin{array}{l}\text { Siembra- } 10 \% \\
\text { cobertura }\end{array}$ & 0,08 & \\
\hline & 4 & 90,4 & Cebada & Período 3 & 0,07 & \\
\hline & 5 & 180,1 & Maíz & Período 1 & 0,2 & \\
\hline & 6 & 59,9 & Soja $1^{\mathrm{a}}$ & Período 1 & 0,08 & \\
\hline & 7 & 108,1 & Soja $1^{a}$ & Período 1 & 0,08 & \\
\hline & $\begin{array}{l}\text { Área cerros } \\
\text { y pajonales }\end{array}$ & 53,6 & - & - & 0,00003 & \\
\hline \multirow{8}{*}{$19 / 12 / 2012$} & 1 & 34,2 & Soja $1^{a}$ & Período 1 & 0,08 & \multirow{8}{*}{0,10} \\
\hline & 2 & 26,7 & Soja $1^{\text {a }}$ & Período 1 & 0,08 & \\
\hline & 3 & 13,5 & Soja $1^{\text {a }}$ & Período 1 & 0,08 & \\
\hline & 4 & 90,4 & Barbecho (rastrojo de cebada) & & 0,08 & \\
\hline & 5 & 180,1 & Maíz & Período 2 & 0,19 & \\
\hline & 6 & 59,9 & Soja $1^{\text {a }}$ & Período 2 & 0,07 & \\
\hline & 7 & 108,1 & Soja $1^{\text {a }}$ & Período 2 & 0,07 & \\
\hline & $\begin{array}{l}\text { Área cerros } \\
\text { y pajonales }\end{array}$ & 53,6 & - & - & 0,00003 & \\
\hline \multirow{8}{*}{ 28/12/2012 } & 1 & 34,2 & Soja $1^{\text {a }}$ & Período 1 & 0,08 & \multirow{8}{*}{0,10} \\
\hline & 2 & 26,7 & Soja $1^{\mathrm{a}}$ & Período 1 & 0,08 & \\
\hline & 3 & 13,5 & Soja $1^{\text {a }}$ & Período 1 & 0,08 & \\
\hline & 4 & 90,4 & Barbecho (rastrojo de cebada) & - & 0,08 & \\
\hline & 5 & 180,1 & Maíz & Período 2 & 0,19 & \\
\hline & 6 & 59,9 & Soja $1^{\text {a }}$ & Período 2 & 0,07 & \\
\hline & 7 & 108,1 & Soja $1^{\text {a }}$ & Período 2 & 0,07 & \\
\hline & $\begin{array}{l}\text { Área cerros } \\
\text { y pajonales }\end{array}$ & 53,6 & - & - & 0,00003 & \\
\hline
\end{tabular}

